\title{
Single Molecule Fluorescence Spectroscopy and Imaging: \\ Advanced Methods and Applications in Life Sciences
}

\section{Dissertation}

\author{
for the award of the degree \\ "Doctor of Philosophy (Ph.D.)" \\ Division of Mathematics and Natural Sciences \\ of the Georg-August-Universität Göttingen \\ within the doctoral program \\ Physics of Biological and Complex Systems \\ of the Göttingen Graduate School for Neurosciences, Biophysics, and \\ Molecular Biosciences (GGNB) of the \\ Georg-August-University School of Science (GAUSS)
}

submitted by

Arindam Ghosh

from Kolkata, India

Göttingen, 2020 


\section{Thesis Advisory Committee:}

Prof. Dr. Jörg Enderlein

Third Institute of Physics - Biophysics, Georg-August-University, Göttingen

Prof. Dr. Reinhard Jahn

Department of Neurobiology, Max Planck Institute for Biophysical Chemistry, Göttingen

Prof. Dr. Fred Wouters

Molecular and Cellular Systems Lab, Institute for Neuropathology, University Medical Center, Göttingen

\section{Members of the Examination Board:}

Prof. Dr. Jörg Enderlein (Referee 1)

Third Institute of Physics - Biophysics, Georg-August-University, Göttingen

Prof. Dr. Reinhard Jahn

Department of Neurobiology, Max Planck Institute for Biophysical Chemistry, Göttingen

Prof. Dr. Fred Wouters (Referee 2)

Molecular and Cellular Systems Lab, Institute for Neuropathology, University Medical Center, Göttingen

Prof. Dr. Kai Tittmann

Schwann Schleiden Research Center, Department of Molecular Enzymology, Georg-AugustUniversity, Göttingen

Dr. Andreas Neef

Campus Institute for Dynamics of Biological Networks, Max Planck Institute for Experimental Medicine, Göttingen

Prof. Dr. Ralph Kehlenbach

Institute for Molecular Biology, University Medical Center, Göttingen

Date of Oral Examination : 01.12.2020 


\section{Affidavit}

Hereby, I declare that the presented thesis has been written independently and with no other sources and aids than quoted. The presented work is a cumulative dissertation where multiple peer-reviewed published reports are reproduced with permission which are listed below.

\section{List of related publications}

Arindam Ghosh, Alexey I. Chizhik, Narain Karedla and Jörg Enderlein. Grapheneand metal-induced energy transfer : From single-molecule imaging to live cell nanoscopy with (sub)-nanometre axial resolution. Nature Protocols (protocol invitation in revision).

Arindam Ghosh, Jörg Enderlein, and Eugenia Butkevich. Dimerization of human drebrin-like protein governs its biological activity. Biochemistry, 59(16):1553-1558, 2020.

Steffen Mühle, Man Zhou, Arindam Ghosh, and Jörg Enderlein. Loop formation and translational diffusion of intrinsically disordered proteins. Physical Review E, 100(5):05 $2405,2019$.

Arindam Ghosh, Akshita Sharma, Alexey I Chizhik, Sebastian Isbaner, Daja Ruhlandt, Roman Tsukanov, Ingo Gregor, Narain Karedla, and Jörg Enderlein. Graphene-based metal-induced energy transfer for sub-nanometre optical localization. Nature Photonics, 13(12):860-865, 2019.

Arindam Ghosh, Narain Karedla, Jan Christoph Thiele, Ingo Gregor, and Jörg Enderlein. Fluorescence lifetime correlation spectroscopy: Basics and applications. Methods, 140:32-39, 2018.

Arindam Ghosh, Sebastian Isbaner, Manoel Veiga-Gutiérrez, Ingo Gregor, Jörg Enderlein, and Narain Karedla. Quantifying microsecond transition times using fluorescence lifetime correlation spectroscopy. The journal of physical chemistry letters, 8(24):6022-602 8, 2017. 


\section{Summary}

The visualization of biological structures down to the molecular length scale has been recently made possible by the development of super-resolution fluorescence microscopy. These techniques now routinely resolve biological structures down to a few nanometers. Various super-resolution techniques have been developed, the most successful being Stimulated Depletion Emission (STED) microscopy and Single Molecule Localization Microscopy (SMLM). In what follows, I will focus on the latter class of techniques which is based on the fact that a single molecule image allows for localizing the molecule with a much higher accuracy than the diffraction limit of resolution of the used microscope. However, a big challenge of SMLM is to achieve a similar super-resolution along the optical axis of a microscope. For this purpose, metal-induced energy transfer (MIET) imaging was recently introduced as an elegant method for axially localizing fluorophores with nanometer precision. The underlying principle of MIET is based on an electromagnetic near-field-mediated energy transfer from an excited fluorescent emitter (donor) to a thin planar metal film (acceptor). This energy transfer leads to a distance-dependent modulation of an emitter's fluorescence lifetime (quenching), that can be easily measured with conventional fluorescence lifetime measurement techniques. The power of MIET is that it works with any fluorophore, and it only requires a conventional fluorescence lifetime imaging (FLIM) microscope. In this thesis, I present a powerful modification and further development of MIET, that is called graphene-induced energy transfer (GIET). GIET replaces the metal film of MIET with a single sheet of graphene which reduces the quenching range by one order of magnitude, leading to a tenfold improvement in axial resolution. This enables the localization of fluorophores with sub-nanometer accuracy. We demonstrate the potential of GIET by quantifying inter-leaflet distances in supported lipid bilayers (SLBs) and discuss the potential of the technique particularly in membrane biophysics applications. The second line of this thesis is devoted to the complementary topic of fast molecular dynamics. While super-resolution microscopy succeeds in resolving structural details with nanometer resolution, it is too slow for temporally resolving the fast dynamics of the observed molecules. For this purpose, spectroscopic techniques such as single molecule fluorescence spectroscopy (SMFS) have become an important tool that can resolve molecular dynamics down to timescales of nanoseconds. In my thesis, I focus on fluorescence lifetime correlation spectroscopy (FLCS), an advanced variant of fluorescence correlation spectroscopy (FCS). Using FLCS, I could disentangle two emission states in an autofluorescent protein that have otherwise highly overlapping spectra, and I could quantify the microsecond switching rates between these two states. As compared to other existing methods, FLCS offers the unique advantage of probing such fast switching kinetics with nanosecond temporal resolution under equilibrium conditions at room 
temperature, making it the method of choice for similar studies of complex luminescent emitters. Finally, I will also present another study where I utilized advanced FCS for studying protein self-assembly. In summary, my thesis presents several advanced methods in SMLM and SMFS which significantly enhance the spatial and temporal resolution at the single molecule level. I believe that the presented methods will find a wide range of applications in the life sciences. 


\section{Acknowledgements}

Although no amount of words are sufficient, but I still would like to thank my parents who ensured that I receive a good education even if they stayed with half-filled stomachs during the last days of a month. My uncle, who passed away last year and my aunt did everything to support my parents for my well-being. My girlfriend Puja, who has been with me for eight long years now, believed in me and stood by me in all ups and downs. My interest in Science began in childhood while I was reading science-fiction stories centered around an iconic character Professor Shonku created by the only Oscar winning filmmaker from India, Satyajit Ray. I am thankful to them for sowing the first seeds of imagination in my curious mind.

I am and shall be forever indebted to Jörg Enderlein, who not only supervised me during this thesis work but also shaped me to be a better thoughtful person. He picked me up from a 'pond' to try me out in an 'ocean', the big field of scientific research. During this time, he believed in me and gave me the freedom at work which immensely helped in my development as a researcher. When I came in this town as a stranger, I was fortunate enough to have met three persons, Narain Karedla, Sebastian Isbaner, and Soheil Mojiri, who are still an inspiration to me. I did not have the fortune to witness Elvis performing but I have seen Narain coding, Sebastian sitting all throughout the day in the same chair to solve a problem and Soheil working for hours in the dark optical lab on his microscope. I have learnt from all of them, immensely. Alongside, the fun times spent with them with coffee and our intense table soccer matches will remain memorable. Our senior scientist, Ingo Gregor, can also be called as a 'walking encyclopedia'. At times, I have found its better to ask Ingo than to google some question for getting a quick and effective solution. I would like to acknowledge Alexey, Anna, Roman, Steffen, Jan, Akshita, Aditya, Christoph, Oleksii, Tao, Hongje, Nazar and Subhabrata to make me feel at home here and the good times we spent together will always remain in my heart. I also heartily acknowledge Christoph and Soheil for critically reading this thesis. I would also like to thank Thomas Geiling, Regina Wunderlich and Ursula Hahn-Woergoetter for their continuous support. Time has flown by rather quickly. Many friends close to my heart, Rangeet, Shoubhik, Piyali, Shekhar, Sufi whom I enjoyed spending time in my leisure have left this town for future ventures. I am thankful to all of them. 
The woods are lovely, dark and deep, But I have promises to keep, And miles to go before I sleep, And miles to go before I sleep.

Robert Frost 
To Bapi - my father, best friend, philosopher and guide. 



\section{Contents}

List of Figures $\quad$ xi

1 Introduction 1

2 Background and Scope $\quad 6$

2.1 Fluorescence and fluorophores . . . . . . . . . . . . . . . . . 6

2.2 Fluorescence spectroscopy . . . . . . . . . . . . . . . . . . . . . . 11

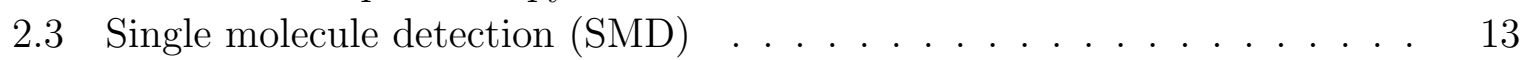

2.4 Single molecule fluorescence spectroscopy (SMFS) . . . . . . . . . . . . . . 13

2.4.1 Fluorescence correlation spectroscopy (FCS) . . . . . . . . . . 15

2.4.2 Fluorescence lifetime correlation spectroscopy (FLCS) . . . . . . . 17

2.5 Fluorescence microscopy . . . . . . . . . . . . . . . . . . . . 20

2.6 Super-resolution fluorescence microscopy . . . . . . . . . . . . . . . . . . . . . . . . . . . . . . 23

2.6.1 Diffraction barrier of optical resolution . . . . . . . . . . . . . . . 23

2.6.2 Super-resolution imaging techniques . . . . . . . . . . . . . . . . . 23

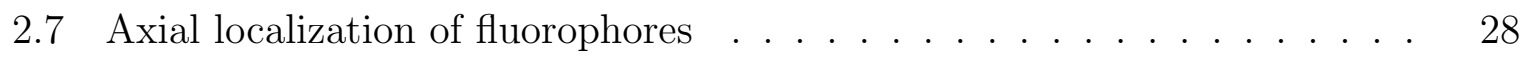

2.7.1 Metal-induced energy transfer (MIET) . . . . . . . . . . . . . . . . . 31

2.7.2 Graphene-induced energy transfer (GIET) . . . . . . . . . . . . . 32

3 Fluorescence Lifetime Correlation Spectroscopy (FLCS) 33

3.1 Manuscript: Quantifying microsecond transition times using fluorescence lifetime correlation spectroscopy . . . . . . . . . . . . . 33

3.2 Manuscript: Fluorescence lifetime correlation spectroscopy : Basics and applications ............................. 56

4 Metal- and Graphene-Induced Energy Transfer (MIET and GIET) 65

4.1 Manuscript: Graphene-based metal-induced energy transfer for sub-nanometer optical localization . . . . . . . . . . . . . . . . 65

4.2 Manuscript: Graphene- and metal-induced energy transfer : from singlemolecule imaging to live cell nanoscopy with (sub)-nanometer axial resolution 89

5 Single Molecule Spectroscopy and Imaging for Studying Protein Structure and Dynamics

5.1 Manuscript: Dimerization of human drebrin-like protein governs its biological activity . . . . . . . . . . . . . . . . . . . . 125

5.2 Loop formation dynamics and diffusion of disordered proteins . . . . . 141 
6 Conclusion and Outlook

Bibliography

Curriculum Vitae

164 


\section{List of Figures}

2.1 Example of fluorescence in nature . . . . . . . . . . . . . . . 6

2.2 Jablonski diagram . . . . . . . . . . . . . . . . . . . . . 7

2.3 Stokes shift of fluorescence emission of a standard fluorophore . . . . . 8

2.4 Enhanced green fluorescent protein (EGFP) and its chromophore . . . . 10

2.5 Example of an organic dye: Rhodamine 6G . . . . . . . . . . . . . 11

2.6 Scheme of Förster resonance energy transfer (FRET) and photo-induced electron transfer $(\mathrm{PET}) \ldots \ldots \ldots$. . . . . . . . . . . . . . . . . 14

2.7 Concept of fluorescence correlation spectroscopy (FCS) . . . . . . . . . 16

2.8 Time-tagged time-resolved (TTTR) counting scheme . . . . . . . . . 18

2.9 Working principle of FLCS . . . . . . . . . . . . . . . . . 19

2.10 Wide-field and confocal illumination . . . . . . . . . . . . . . 22

2.11 Concept of stimulated emission depletion (STED) microscopy . . . . . . 24

2.12 Concept of switching-based super-resolution microscopy . . . . . . . . . 26

2.13 Metal- and graphene-induced energy transfer (MIET and GIET) . . . . . 31 


\section{Glossary of Acronyms}

ACF Autocorrelation Function

ADP Adenosine Diphosphate

APD Avalanche Photodiode

ATP Adenosine Triphosphate

BP FPALM Bi-plane Fluorescent Photoactivated Localization Microscopy

CCF Crosscorrelation Function

CLSM Confocal Laser Scanning Microscope

DNA Deoxyribonucleic Acid

DOPC Dioleoylphosphatidylcholine

dSTORM Direct Stochastic Optical Reconstruction Microscopy

DTT Dithiothreitol

EGFP Enhanced Green Fluorescent Protein

EMT Epithelial to Mesenchymal Transition

EPR Electron Paramagnetic Resonance

FCS Fluorescence Correlation Spectroscopy

FCCS Dual Colour Fluorescence Crosscorrelation Spectroscopy

FLIM Fluorescence Lifetime Imaging Microscopy

FLCS Fluorescence Lifetime Correlation Spectroscopy

FP Fluorescent Protein

FRET Förster Resonance Energy Transfer

GIET Graphene-Induced Energy Transfer

GFP Green Fluorescent Protein

HBI p-Hydroxybenzylidene-Imidazolidone 
ISM Image Scanning Microscopy

iPALM Interferometric Photoactivated Localization Microscopy

LS-FCS Line-Scan Fluorescence Correlation Spectroscopy

MEA Mercaptoethylamine. $\mathrm{HCl}$

MIET Metal-Induced Energy Transfer

NMR Nuclear Magnetic Resonance

PAINT Point Accumulation for Imaging in Nanoscale Topography

PALM Photoactivated Localization Microscopy

PET Photo-Induced Electron Transfer

PMT Photo-Multiplier Tube

PSF Point Spread Function

SAXS Small Angle X-ray Scattering

SIM Structured Illumination Microscopy

SMLM Single-Molecule Localization Microscopy

SMS Single-Molecule Spectroscopy

SNR Signal-to-Noise Ratio

STED Stimulated Emission Depletion

STORM Stochastic Optical Reconstruction Microscopy

SUV Small Unilamellar Vesicle

TCSPC Time-Correlated Single Photon Counting

TIRFM Total Internal Reflection Fluorescence Microscopy

TTTR Time-Tagged Time-Resolved 



\section{Chapter 1}

\section{Introduction}

$\mathbf{E}$ physics (optics). The interaction between light and matter is the foundation of all optical techniques such as optical microscopy or optical spectroscopy. However, the techniques that have become particularly important for the life-science are all based on fluorescence. This is due to the unprecedented specificity (only specific organic dyes or proteins exhibit fluorescence, with specific absorption and emission properties) and sensitivity (down to single molecules) of fluorescence. Even more, fluorescence offers the unique advantage of observing biological processes under native conditions and in living cells, which offers the possibility to answer many fundamental biological questions that had been unanswered over centuries before. As Richard Feynman said: It is very easy to answer many of these fundamental biological questions; you just look at the thing!

The quest for looking at the 'invisible' world began with the invention of the first compound microscope by Galileo Galilei in 1609. In 1676, Antonie van Leeuwenhoek built a microscope with one lens that he used to examine blood cells, yeast, and insects. However, the resolution of these optical systems, that is the smallest distance between two points in a specimen that can be distinguished, was dependent on how craftily a microscope was built. This changed almost 200 years later, when Ernst Karl Abbe developed the theoretical basis of modern microscopy and derived his famous relation that couples the resolution of a microscope with the wavelength of light (diffraction limit of optical resolution). Historically, developments in light microscopy have always been motivated by the desire for obtaining a detailed understanding of the structural organization and function of biological systems. In this connection, inventions such as Köhler illumination (August Köhler, 1893), phase contrast microscopy (Frits Zernike, 1933), and differential interference contrast microscopy (Georges Nomarski, 1952) deserve special mention. 
The realization of the first fluorescence microscope dates back to 1908 when August Köhler and Henry Siedentopf built a first prototype of such a microscope. Technical advancements followed, using fluorescent dyes or pigments such as porphyrins as probes. Modern fluorescence microscopy gained huge momentum with the discovery of the green fluorescent protein (GFP) in 1962 by Shimomura and colleagues during the purification of the bioluminescent protein aequorin from Aequorea victoria [1]. Most importantly, thirty years after its discovery, in 1992, the gene that codes for GFP was cloned, and the utility of GFP as a fluorescent marker tagged to biological structures in vivo was demonstrated [2]. This paved the way to observe structural organization and dynamics in living cells and tissue. This was followed by the emergence of novel synthetic dye molecules that can be chemically or enzymatically attached to structures of interest. In parallel, technical developments in diffraction-limited optical systems made also significant progress, leading to the modern widefield and confocal fluorescence research microscopes. Taken together, an exponential rise of fluorescence microscopy applications in bio-imaging can be seen in the 1990s, making fluorescence microscopy one of the most important techniques in the modern life sciences.

Although fluorescence microscopy gradually became a routine technique, it was still limited in spatial resolution by the diffraction of light, which meant that one could not resolve two emitters positioned closer than roughly $200 \mathrm{~nm}$ (when using green light). While a diffraction-limited system can be conveniently used to visualize single cells (few microns in size), it is not capable to resolve details in, for example, a single virus, a protein, or a DNA molecule, all having sizes between 1-100 nanometers. Thus, overcoming this diffraction barrier of spatial resolution became a major goal in the further development of optical microscopy. A first and most important step of overcoming the resolution limit of optical microscopy was done in 1994 by S.W. Hell an colleagues, who developed stimulated emission depletion (STED) [3]. Later, a second class of super-resolution microscopy techniques was developed, namely single molecule localization microscopy (SMLM), which is based in imaging and localizing individual fluorescence molecules. The development of SMLM itself was based on the earlier successful realization of single molecule detection, first with the work by W.E. Moerner, L. Kador in 1989 [4] and M. Orrit and J. Bernard in 1990 [5] at cryogenic temperatures, and then later and most importantly with the successful detection of single molecules in solution at room temperature by R.A. Keller using a modified flow cytometer [6]. The class of SMLM techniques comprises photoactivated localization microscopy (PALM) [7], stochastic optical reconstruction microscopy (STORM) [8], direct STORM (dSTORM) [9], and point accumulation for imaging in nanoscale topography (PAINT) [10]. These methods achieve a lateral resolution down to a few nanometers. They enable the visualization of biological structures with unprecedented detail down to molecular length scales, serving as an ideal tool for non-invasive 
structural biology. It should be mentioned that alongside super-resolution fluorescence microscopy, several other methods are commonly utilized for investigation of structural biology. Among them are transmission electron microscopy [11], scanning electron microscopy [12], x-ray crystallography [13], and cryo-electron microscopy [14]. Although all these methods are capable of discerning molecular and atomic structures of biomolecules with Angstrom resolution, their major drawback is their invasive nature inhibiting their application in living systems.

SMLM techniques, namely PALM, STORM, dSTORM and PAINT, are optimized for achieving maximum lateral spatial resolution. In a diffraction-limited optical microscope, the spatial resolution along the optical axis is $\sim 700 \mathrm{~nm}$. In order to achieve superresolution also along the optical axis, several SMLM imaging schemes have been developed, such as astigmatic imaging [15], bi-plane imaging [16], or wavefront shaping [17]. Similar to a diffraction-limited microscope, the achievable localization accuracy of these schemes is typically three to five times worse as compared to the achievable lateral resolution. There exist few exceptional approaches based on optical interferometry such as iPALM $[18,19]$ or isoSTED [20, 21], that achieve an axial resolution equal or even better than the lateral resolution, but for the price of elevated technical complexity that currently limits their wide usage. In this vein, our group developed metal-induced energy transfer (MIET) as an easy to implement and to use alternative [22] for axial localization of fluorophores with nanometer accuracy. MIET relies on electrodynamic near-field-mediated energy transfer from an optically excited fluorescent molecule (donor) to a thin planar metal film of $\sim 10-15 \mathrm{~nm}$ thickness. This energy transfer leads to a distance-dependent modulation of the excited-state fluorescence lifetime, which can be easily determined by conventional fluorescence-lifetime measurement techniques. The measured excited-state lifetime of a fluorescent molecule can then be converted into an axial distance value by using a theoretical model of the fluorophore-metal interaction. Modulation of fluorescence lifetime near a metal layer was extensively studied by Kuhn and Drexhage in the 1970s, see e.g. ref. [23]. This phenomenon can be quantitatively described by considering the fluorescent emitter to be an ideal oscillating electric dipole emitter, and to model the interaction of this emitter with the metal using classical Maxwell's electrodynamics. Recently, we have demonstrated that by using a single sheet of graphene instead of a metal film, one can increase the achievable axial resolution by nearly tenfold, thus enabling sub-nanometer optical localization of fluorophores. This advanced variant of MIET was named graphene-induced energy transfer (GIET) [24]. The current work presents the theoretical framework of GIET imaging and its application for determining axial distances with sub-nanometer resolution.

While understanding molecular architectures is one of the central goals of fluorescence bio-imaging, another one is resolving the fast dynamics of biomolecules on timescales 
down to nanoseconds. This dynamics is often crucial for their biological function. For example, timescales of enzyme turnover, electron transfer reactions, protein folding, protein translation, and many other processes take place on time-scales between few nanoseconds to tens of milliseconds. For a precise quantification of processes on these timescales, fluorescence spectroscopy at the single molecule level has emerged as a powerful tool [25] that enables the detection of molecular sub-species that would be otherwise be invisible in classical ensemble measurements. This set of techniques, generally termed single molecule fluorescence spectroscopy (SMFS) allows us to follow reaction pathways which are otherwise impossible to resolve with ensemble techniques. Here, single molecule Förster resonance energy transfer (sm-FRET) is the most widely used SMFS technique for investigating bio-molecular dynamics both in vitro and in vivo [26]. This method relies on the distance-dependent energy transfer from an excited 'donor' fluorophore to an 'acceptor' fluorophore and has been successfully employed to probe protein folding, enzymatic reactions, binding-unbinding kinetics, and many more [27-29]. Fluorescence correlation spectroscopy (FCS) is another frequently utilized SMFS technique [30]. It is based on the temporal correlation of fluorescence intensity fluctuations recorded in the small detection volume of a confocal microscope from fluorophores at nanomolar concentrations. These fluorescence intensity fluctuations typically originate from the Brownian motion of the dye molecules, their photophysics, from chemical reactions, intramolecular conformational dynamics etc. For the FCS correlation analysis, a recorded fluorescence intensity time trace is point-wise multiplied with a time-shifted replica of itself, repeated for all possible time-shift values (lag times), which results in the so-called second-order autocorrelation function (ACF). On the microsecond timescale, the ACF is shaped by fast photophysical processes, such as triplet state dynamics or cis-trans isomerization, while on the millisecond timescale, lateral diffusion of molecules out of the detection volume leads to its long-time decay. An important application of FCS is its combination with photo-induced electron transfer (PET-FCS) for probing the conformational dynamics in polymer chains [31]. It should be mentioned that there are a huge number of non-optical methods that can also provide information of intra- and intermolecular dynamics on timescales from nanoseconds to milliseconds, namely nuclear magnetic resonance (NMR) spectroscopy [32, 33], electron paramagnetic resonance (EPR) [34], small angle x-ray scattering (SAXS) [35], or neutron scattering [36]. They all can provide quantitative information about three dimensional structure, topology, and dynamics of molecules in solution and in the solid state. For example, NMR relaxation dispersion experiments have provided crucial insights into the conformational inter-conversion of bio-molecules on a timescale of micro- to milliseconds. In particular, dynamics and interactions in protein chains is studied using this NMR. However, the sensitivity of all the mentioned methods is by orders of magnitude worse than that of SMFS, requiring 100 micromolar to molar sample concentrations. 
Classical FCS relies on fluctuations in the recorded fluorescence intensity signal, encoding information about conformational dynamics, photophysical transitions etc. However, many complex luminescent emitters such as fluorescent proteins have multiple emission states leading to multiexponential fluorescence decays and rapid fluctuations between different excited-state lifetime states. However, conventional FCS is insensitive to changes in fluorescence lifetime. Thus, an advanced extension of FCS was developed, fluorescence lifetime correlation spectroscopy (FLCS) [37], that enables to measure fast intramolecular transitions that lead to fast lifetime fluctuations. The core idea of FLCS is the calculation of lifetime-dependent ACFs by weighing the recorded photons with statistical filter functions obtained from distinctive decay patterns of each fluorescent state. A cross-correlation analysis then provides information about transitions between lifetime states with a temporal resolution down to nanoseconds. We demonstrated the potential of FLCS by quantifying microsecond transition rates between two emission states of the prototypical fluorescent protein enhanced GFP (EGFP) [38].

This work is organized as follows: in the next chapter, I provide a comprehensive overview on the background of fluorescence, fluorophores, fluorescence microscopy, and fluorescence spectroscopy. Next, I briefly discuss the scope of this thesis and introduce its relevant topics. In the third chapter, I present two peer-reviewed and published manuscripts which provide the theoretical framework of FLCS, and its application for quantifying rapid photophysical transitions in EGFP. The fourth chapter encompasses the theoretical framework of GIET and its utilization for single molecule localization and the determination of the distance between two leaflets in a supported lipid bilayer. The working principle of GIET and MIET imaging in general is presented in a published review. Finally yet importantly, the fifth chapter presents some further biophysical applications of various FCS-based SMFS techniques as well as fluorescence lifetime imaging microscopy (FLIM) for investigating protein structure and dynamics. 


\section{Chapter 2}

\section{Background and Scope}

\subsection{Fluorescence and fluorophores}

Fluorescence is a molecule's ability to emit light after being excited by light of a suitable wavelength [39]. A beautiful example of fluorescence in nature can be seen in tiny Brazilian frogs (Brachycephalus ephippium) where the backs and heads of the frogs glow intensely under ultra-violet (UV) light [40] (Figure 2.1). A molecule exhibiting fluorescence is generally termed a fluorophore. It is characterized by its capability of absorbing light of a specific wavelength, and of re-emitting the absorbed energy again as light at a longer but specific wavelength. Fluorophores, which are mostly organic molecules, have electronic states that can be divided into singlet and triplet states where all electrons are spin-paired or one set of electron spins is unpaired, respectively. Upon excitation with light, an electron in the fluorophore jumps from an electronic ground state $\left(S_{0}\right)$ to one

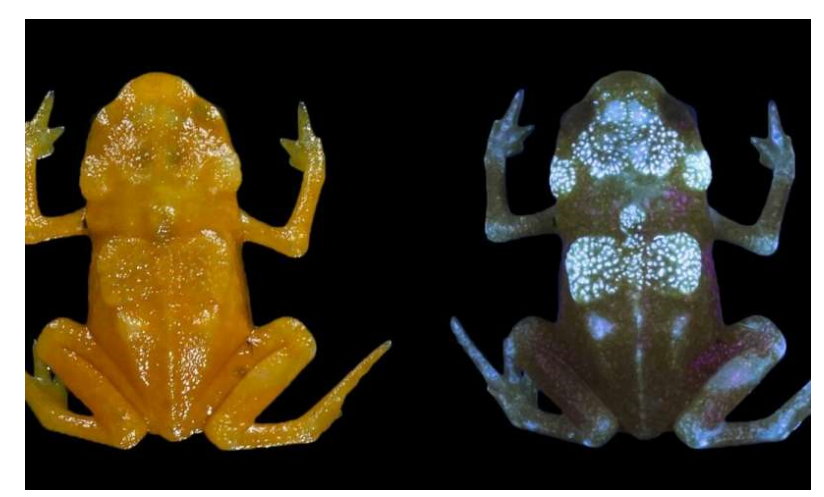

Figure 2.1: Fluorescence observed in the Brazilian frog Brachycephalus ephippium. Left panel visualizes a colored photograph of B. ephippium and right panel shows a fluorescent image of the same under illumination with UV light as described in [39]. 


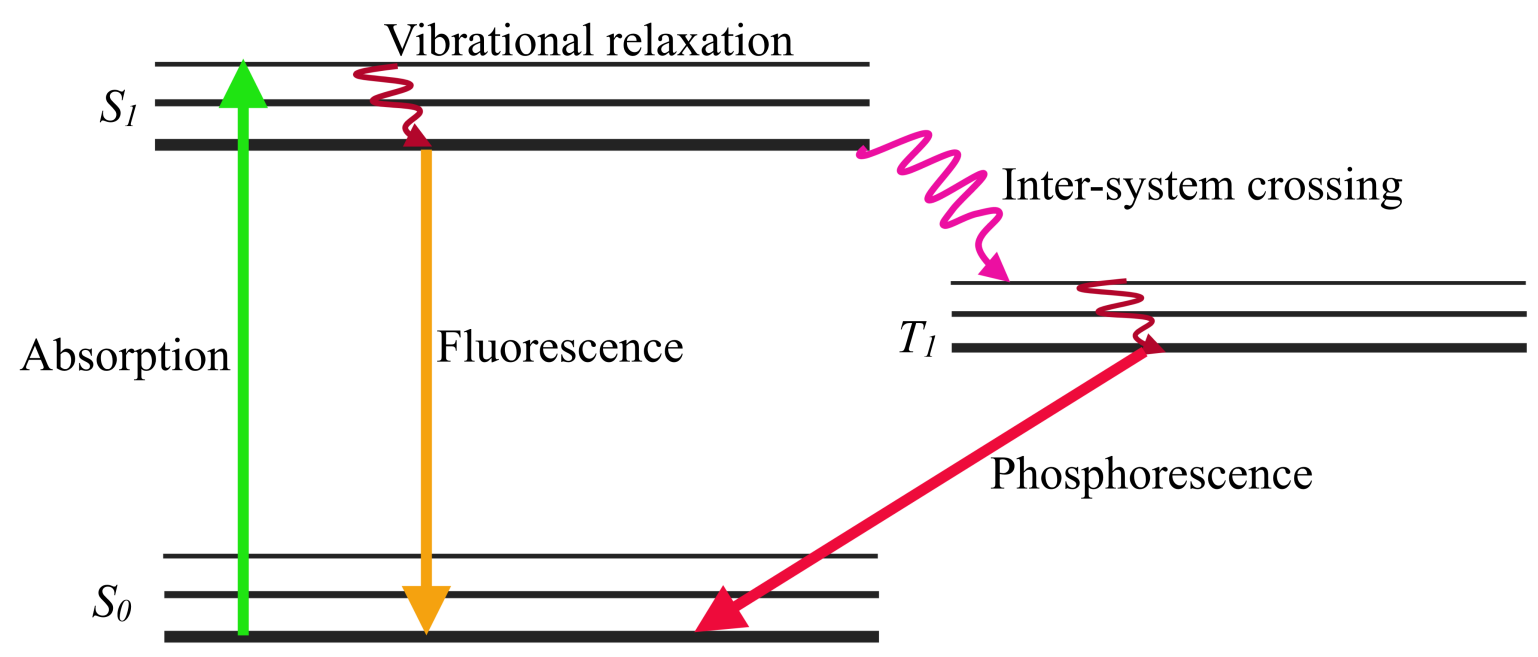

Figure 2.2: Figure shows a simplified Jablonski diagram. A fluorophore absorbs light of a suitable wavelength (shown in green arrow) in its electronic ground state $S_{0}$ and is excited to higher vibrational levels of the first excited state $S_{1}$. Via vibrational relaxation (in brown curved arrow) the molecule returns to the lowest vibrational level of $S_{1}$. From here, the molecule can return to the ground state, either radiatively via the emission of a photon which is manifested as fluorescence (orange arrow), or non-radiatively via inter-molecular collisions. Alternatively, the molecule can switch to a triplet state $T_{1}$ via inter-system crossing (shown in pink curved arrow). Following this, the fluorophore decays to the lowest vibrational level of $T_{1}$ via vibrational relaxation and finally returns to the ground state $S_{0}$ through phosphorescence (shown as red arrow).

of the multiple vibrational levels of an excited electronic singlet state $\left(S_{n}\right)$ (see Jablonski diagram in Figure 2.2). This is almost an instantaneous process occurring at a timescale of $\sim 10^{-15}$ seconds. If a higher electronic state is excited, it quickly relaxes to the lowest (first) excited state in a process called internal conversion $\left(10^{-11}\right.$ to $10^{-9}$ seconds). Any excess vibrational energy is also given away by vibrational relaxation (within $10^{-12}$ to $10^{-10}$ seconds), after which one finds the molecule in the lowest-lying vibrational level of its first electronic state $\left(S_{1}\right)$. From this state, the fluorophore returns to the ground state $\left(S_{0}\right)$ by either non-radiative relaxation or by emitting a photon otherwise called fluorescence. The typical timescale of this transition rate is $10^{-10}$ to $10^{-7}$ seconds. It should be noted that excitation of a molecule into its excited state is usually done via one-photon excitation (i.e. the absorption of one photon of suitable energy), but can also be achieved via multi-photon excitation (i.e. by the quasi-simultaneous absorption of several photons of suitable lower energy). Besides returning to the singlet ground state $S_{0}$, there is a small chance for a transition into the so-called triplet state which requires 
the reversal of the excited electron's spin (inter-system crossing). Then, the molecule is found in its the first excited triplet state $\left(T_{1}\right)$. Inter-system crossing is a "forbidden" process (violating momentum conservation) although the triplet state is of lower electronic energy than the excited singlet state. It can nonetheless happen due to spin-orbit coupling, relaxing the spin momentum conservation, but only with a low probability. Hence, it is fairly infrequent in most organic fluorophores (occurring typically once every $\sim 10^{3}$ $S_{1} \rightarrow S_{0}$ transitions). From the triplet state, the molecule can return to the ground state again involving a spin flip, which can be also connected with the emission of a photon (phosphorescence).

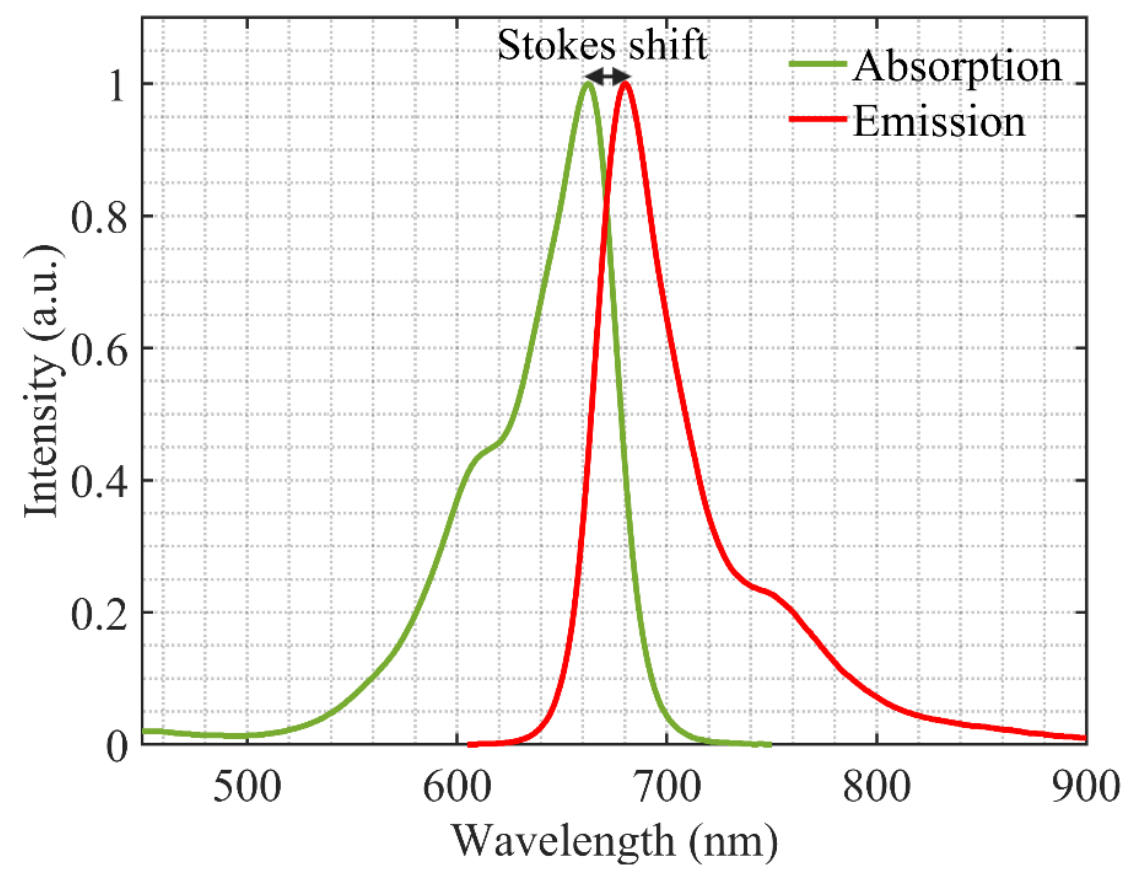

Figure 2.3: The figure shows the absorption and fluorescence emission spectra of the typical organic fluorophore Atto 655. The absorption spectrum is shown in green and the emission spectrum in red. The wavelength difference between absorption and emission maxima is called Stokes shift, as depicted in the plot.

The fluorescence spectrum of a dye molecule is always red-shifted relative to its absorption spectrum. This implies that the molecule emits photons of lower energy at longer wavelengths as compared to the photons absorbed. The underlying cause of the Stokes shift is that some of the energy of the excited fluorophore is lost through inter-molecular collisions that occur during the brief lifetime of the molecule's excited state. This energy is dissipated as heat to the environment (solvent molecules). Figure 2.3 shows the absorption and emission spectra of a typical organic fluorophore, Atto 655 (ATTO-TEC $\mathrm{GmbH}$ ), and the Stokes shift between the spectra. Large values of Stokes shift are important for practical applications of fluorescence because it allows to spectrally separate 
the (strong) excitation light from the (weak) emitted fluorescence. Alongside fluorescence spectrum, other important parameters such as the fluorescence quantum yield and fluorescence lifetime of fluorophores govern their selection criteria for microscopic and spectroscopic applications. The fluorescence quantum yield $(\Phi)$ of a fluorescent molecule is defined as the ratio of the number of emitted fluorescence photons to the number of absorbed photons. Mathematically, it is defined by

$$
\Phi=\frac{k_{r}}{k_{r}+k_{n r}}
$$

where $k_{r}$ and $k_{n r}$ are the radiative and non-radiative decay rate constants, respectively. Non-radiative processes include internal conversion, intra- and inter-molecular quenching or intersystem crossing. Ideally, one would like to work with fluorophores having a quantum yield value of $\Phi=1$, but most fluorophores used in microscopy and spectroscopy show values between 0.2 and 1.0. To obtain high quantum yield values, a molecule should have a relatively rigid structure minimizing rotations or vibrations of side groups which prevent radiationless deactivation, ensuring a low intersystem crossing rate, and charge transfer transitions owing to conjugated electron donor and acceptor groups. A second important parameter is fluorescence lifetime $\left(\tau_{f}\right)$ of a fluorophore which is defined as the average time a fluorophore spends in its excited singlet state $S_{1}$.

$$
\tau_{f}=\frac{1}{k_{r}+k_{n r}}
$$

For an ensemble of excited molecules, $\tau_{f}$ is the characteristic time with which the number of molecule still in their excited state decreases. Since the number of excited fluorophores is proportional to the momentous fluorescence intensity $I(t)$, this leads to a mono- exponential decay of the observable fluorescence intensity with decay time $\tau_{f}$, similar to a radioactive decay:

$$
I(t)=I_{0} e^{-t / \tau_{f}}
$$

Lifetime of a fluorescent molecule is sensitive to subtle changes in the local environment such as alterations in local charge, solvent $\mathrm{pH}$, or viscosity. Hence, fluorescence lifetime is a non-trivial observable which is routinely exploited to study various chemical and biochemical systems.

Fluorophores normally contain delocalized electrons in conjugated double bonds. Naturally occurring fluorescent species such as proteins and nucleic acids possess delocalized electrons in many of their monomeric units. They absorb and emit light in the ultra-violet (UV) region. For example, in proteins, three amino acids having aromatic side chains, 


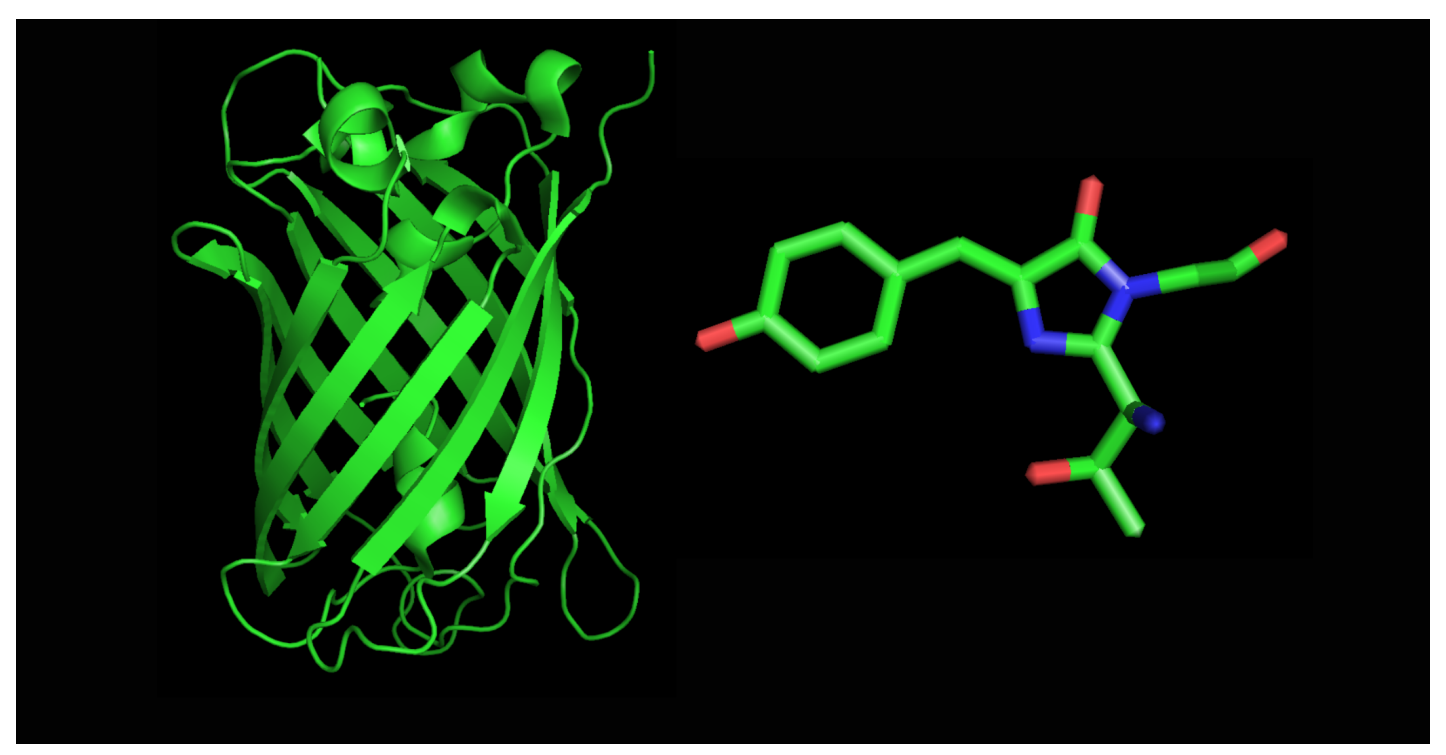

Figure 2.4: EGFP and its chromophore. Secondary structure of EGFP as obtained from x-ray scattering (PDB ID 4EUL) is shown on the left. It contains five $\alpha$ helices and one pleated sheet of eleven $\beta$-strands. The chromophore core $\mathrm{HBI}$ is shown on the right is formed by threonine, tyrosine and glycine residues.

namely tryptophan, tyrosine and phenylalanine, are responsible for their observed autofluorescence [41]. Among these three amino acids, tryptophan shows the highest fluorescence QY and thus dominates the auto-fluorescence of proteins [42, 43]. Cofactors such as nicotinamide adenine dinucleotide (NADH) [44] and flavin adenine dinucleotide (FAD) $[45,46]$, playing important role in cellular metabolism, are also fluorescent. Of all the naturally existing luminescent biomolecules, the most prominent example is the green fluorescent protein (GFP) which was isolated and purified from the jellyfish Aequorea victoria [47]. GFP is composed of 238 amino acid residues $(\sim 27 \mathrm{kDa})$ and exhibits a bright green fluorescence emission upon excitation with blue light. GFP from A. victoria, commonly termed as avGFP, has an excitation peak at $395 \mathrm{~nm}$ and a minor peak at $475 \mathrm{~nm}$, while the emission maximum is at $509 \mathrm{~nm}$. The protein has a quantum yield of 0.79 . GFP is characterized by its signature beta barrel structure which consist of eleven $\beta$-strands in a pleated sheet arrangement, and an $\alpha$-helix with the covalently bound chromophore p-hydroxybenzylidene-imidazolidone (HBI). Several mutants of GFP have been engineered and utilized in cell biology, reporter assays, fluorescence microscopy, or spectroscopy in general. Notable examples include enhanced GFP (EGFP), blue fluorescent protein $(\mathrm{EBFP})$, cyan fluorescent protein (ECFP), yellow fluorescent protein (EYFP), and so on [48-50]. The chromophore and the overall structure of EGFP is shown in Figure 2.6. Data was taken from PDB ID 4EUL [51].

The second class of fluorophores which are routinely used are organic dye molecules. Over the last two decades, the utilization of organic dyes in quantitative fluorescence microscopy has witnessed an exponential rise. A bright, photostable fluorophore with 


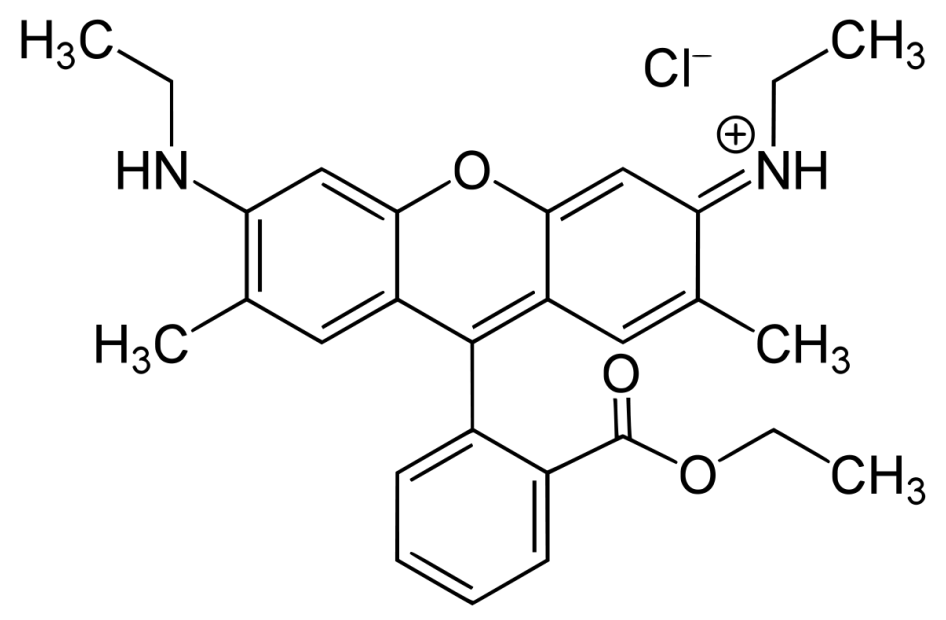

Figure 2.5: Structure of the popular organic fluorophore rhodamine 6G. The dye has an absorption maximum at $530 \mathrm{~nm}$ and two peaks in fluorescence emission at $554 \mathrm{~nm}$ and $602 \mathrm{~nm}$.

high quantum yield is an essential prerequisite for most applications from ensemble spectroscopy to single molecule fluorescence imaging. Commercially available organic dyes exhibit strong absorption and emission bands in the visible region of the electromagnetic spectrum [52-57]. An example of a commercially available organic dye molecule rhodamine $6 \mathrm{G}$ is illustrated in Figure 2.5.

Various strategies are employed to attach fluorophores to molecules and structures of interest. In case of fluorescent proteins (FPs), their gene-encoding domains are cloned together with the gene encoding for the target protein into a plasmid, which is expressed and purified as a fusion protein [58]. Organic dyes are synthesized in various ester forms for conjugation to biomolecules. Some frequently used approaches include NHS-tagging (N-hydroxysuccinimide ester) of a dye molecule to a free amine group at the N-terminus of a protein or a lysine residue [59], or coupling of a dye with a modified maleimide group to the thiol (-SH) group of a a cysteine residue [60]. Immunolabeling is another standard approach. There, a primary antibody is attached to the structure of interest, followed by amplification with a secondary antibody that is tagged with an organic fluorophore [61].

In what follows next, we briefly discuss the utilization of fluorescence-based assays for spectroscopic investigations by monitoring steady-state and time-resolved parameters.

\section{$2.2 \quad$ Fluorescence spectroscopy}

Fluorescence spectroscopy encompasses a set of techniques which are routinely employed for studying a variety of dynamic processes in fluorophore-tagged molecules exploiting the 
changes in fluorescence spectra. The simplest application is determination of molecular concentration of fluorophores from their fluorescence intensity. In particular, steady-state measurements of fluorescence properties such as fluorescence intensity under the emission spectrum or the shape of the spectrum itself provide crucial information on the local environment of the fluorophore. Another useful parameter is fluorescence anisotropy. Excitation with polarized light leads to preferential excitation of molecules whose transition dipole moments are oriented parallel to the electric field vector $\boldsymbol{E}$. This selective photo-excitation results in a polarized fluorescence emission, which also impacts fluorescence lifetime measurements. Mathematically, it is defined as

$$
r=\frac{I_{\|}-I_{\perp}}{I_{\|}+2 I_{\perp}}
$$

where $I_{\|}$and $I_{\perp}$ are the fluorescence intensities measured through linear polarization filters parallel and perpendicular to the excitation polarization, respectively. For fluorophores in solution, the fluorescence anisotropy is lower than for a solid sample due to rotational diffusion, which randomizes the emission dipole orientations of excited molecules over time. Typical rotational diffusion times are on the order of 100 ps for small organic molecules in aqueous solutions, which is much faster than typical fluorescence lifetime $\left(\tau_{f}\right)$ values that are typically in the nanosecond range. In this case, the fluorescence emission is fully depolarized and one finds an anisotropy value $r=0$. On the contrary, if rotational diffusion time is slower than $\tau_{f}$, for example if the fluorophore is tagged to a larger biomolecule, or experiments are done in solvents of higher viscosity or at lower temperatures, one observes a strongly polarized emission. Monitoring fluorescence intensity, emission spectra and anisotropy of fluorophores in a time-resolved manner provides more detailed insights into dynamic processes. Time-resolved emission spectra and anisotropy measurements allow investigation of structural dynamics, molecular motions, excited-state reactions, changes in microviscosity and population heterogeneity [62-66]. These fluorescence spectroscopic measurements are usually done with samples containing micromolar range or higher concentration of fluorophores. This implies that the outcome of these experiments are essentially averaged over a large ensemble of fluorescent molecules. Hence, it is not possible to examine and compare properties of individual fluorescent molecules in a heterogeneous population using the classical approach. However, in most biological and biochemical systems, existence of heterogeneous sub-populations is a standard. For example, detection of rare protein conformations or transient intermediates during the folding-unfolding pathway. In order to detect such events down to the level of individual fluorescent species, we need advanced spectroscopic methods with much higher detection sensitivity. This brings us to the next section focusing on detection of single molecules using fluorescence spectroscopy. 


\subsection{Single molecule detection (SMD)}

The first reports about successful experimental detection of single molecules at cryogenic temperatures were published in two seminal papers, one by W.E. Moerner and L. Kador in 1989 [4], and the other by M. Orrit and J. Bernard in 1990 [5]. Moerner et al. observed the optical absorption spectrum of the single dopant molecule pentacene in a p-terphenyl host crystal at liquid-helium temperatures using frequency-modulation spectroscopy in combination with Stark or ultrasonic modulation. Orrit et al. measured the fluorescence excitation spectrum of single molecules in a pentacene-doped p-terphenyl crystal, also at liquid-helium temperatures. The first successful detection of single rhodamine molecules in aqueous solution at room temperature was done by the group of R.A. Keller using a modified flow [6]. This was the starting point for the innumerable subsequent applications of SMD in the life sciences. Later, E. Betzig used near-field scanning optical microscopy (NSOM) in 1991 for imaging single fluorescent molecules with a spatial resolution beyond the diffraction limit [67]. NSOM is based on placing a light source or detector with dimensions smaller than the optical wavelength $\lambda$ in close proximity $(<\lambda / 50)$ to a sample of interest for generating images with a resolution much below the diffraction resolution limit of classical microscopy. This development was followed by far-field imaging of immobilized single molecules, either using a confocal microscope or a wide-field microscope [68-71]. In particular, this last technical achievement forms today the basis for one of the most powerful techniques of super-resolution microscopy.

\subsection{Single molecule fluorescence spectroscopy (SMFS)}

Single molecule fluorescence spectroscopy (SMFS) has become an important spectroscopic research tool for the life sciences. It enabled the detection and investigation of rare molecular sub-species that would be otherwise conspicuous in ensemble measurements [25]. SMFS allows for measuring time trajectories of molecular conformational dynamics and intermolecular interactions, and to follow reaction pathways that would be otherwise impossible to study with ensemble techniques. The prerequisites of SMFS experiments include bright and photostable fluorophores, fast lasers as excitation source, and advanced high-NA microscopy optics. The main challenge in SMFS experiments is to achieve a high signal-to-noise (SNR) ratio, enabling the detection of a fluorophore's emission above all noise sources such as scattered light or autofluorescence. Over the years, innumerable SMFS techniques have been devised, of which some prominent examples include single molecule Förster resonance energy transfer (sm-FRET) [26], fluorescence correlation spectroscopy (FCS) [30], or photo-induced electron transfer (PET) [31]. FRET 
A

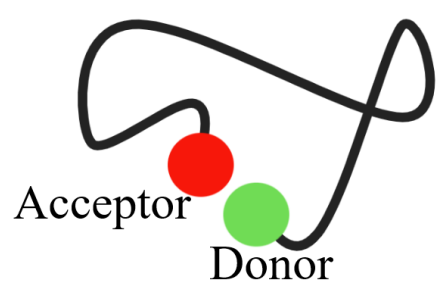

$\mathrm{B}$

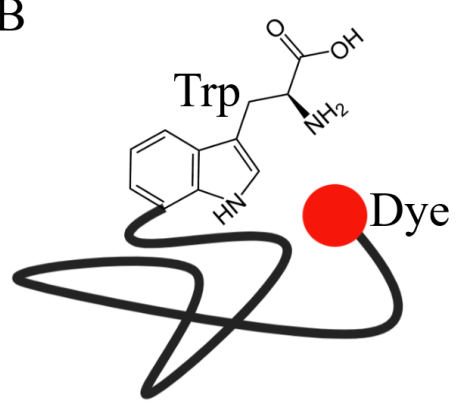

Figure 2.6: Experimental scheme of FRET and PET-FCS. A. shows a polymeric chain labeled with two fluorescent dyes, the donor (green) and the acceptor (red). FRET is extensively used for studying chain dynamics exploiting the strong distance dependence of the electromagnetic dipole-dipole interaction between excited donor and acceptor. B. Schematic of a PET-FCS. A polymer chain, for example a disordered peptide, is tagged with one fluorophore at one end while the other end contains an electron donor that quenches the dye's fluorescence upon direct contact. So far, PET-FCS studies of bio-molecular conformational dynamics were exclusively realized with the oxazine dye Atto 655 as fluorophore and tryptophan or guanine as the quencher.

relies on a distance-dependent energy transfer from a fluorophore (alias donor) molecule to an 'acceptor' fluorescent molecule (Figure 2.12). FRET works over a distance range of 4 to $10 \mathrm{~nm}$ between donor and acceptor. FRET is one of the most widely used techniques for resolving structure, dynamics and interactions of biomolecules, in particular for studying protein folding, enzymatic reactions, or binding kinetics [27-29, 72, 73]. Excellent reviews on are presented in refs. [74-77]. Another method for studying conformational dynamics of biomolecules is photo-induced electron transfer or PET [31] (see Figure 2.12). PET exploits quenching of fluorescence by an electron donor in contact with a fluorophore. As fluorophores, several oxazine and rhodamine based dye molecules (e.g. Atto 655, rhodamine 6G) can be used, which can be quenched by the amino acid tryptophan or the nucleic acid base guanine [78]. PET is extensively used in combination with fluorescence correlation spectroscopy (FCS) for studying conformational dynamics in proteins and oligonucleotides by quantifying the contact rate between the dye and the quencher molecule. FCS correlates temporal intensity fluctuations of the signal detected from a femtoliter confocal detection volume in nanomolar solutions of fluorescently tagged molecules. In the case of PET-FCS, these fluctuations are generated by the reversible contact formation between fluorophore and quencher. A more detailed description of FCS is presented in the next section. 


\subsubsection{Fluorescence correlation spectroscopy (FCS)}

FCS was introduced in the late 70s by Elson, Magde and Webb as a tool for measuring diffusion, concentration, and chemical reaction kinetics of fluorescent or fluorescently labeled molecules at nanomolar concentrations [30, 79]. But the technique did not draw much attention until the 1990s when Manfred Eigen and Rudolf Rigler demonstrated the power of FCS by sorting single fluorescent molecules in solution and its potential applications in diagnostics and evolutionary biotechnology [80]. Any phenomenon causing fluorescence intensity fluctuations in the confocal detection volume will lead to a nontrivial temporal autocorrelation function $(\mathrm{ACF})$ of the recorded fluorescence time trace, which can used to study the fluctuation-inducing process. This correlation function is calculated as

$$
g(\tau)=\langle I(t) I(t+\tau)\rangle_{t}
$$

where $I(t)$ is the recorded fluorescence intensity at time $t, \tau$ is the so-called lag time, and triangular brackets denote averaging over all time values $t$ (see Figure 2.13). The physical meaning of the ACF is that it is proportional to the probability to detect a photon detection event at time $\tau$ if a photon was detected at time zero. Typical examples of processes inducing temporal fluctuations of the measured fluorescence intensity are Brownian motion of molecules through the detection volume, fluorophore photophysics, chemical reactions, or conformational dynamics.

For example, Brownian motion (diffusion) leads to a non-trivial decay of the ACF on a timescale of milliseconds. On very short time scales, while a molecule present in the detection volume does barely move, one observes a high temporal correlation of the fluorescence signal, because a continuous flux of fluorescence photons is excited and detected from the molecule. As soon as the molecule diffuses out of the detection volume, this correlation gradually decays, until the ACF reaches its offset value determined by physically uncorrelated photons. Thus, the decay of of the ACF $g(\tau)$ with increasing lag time $\tau$ will be proportional to the diffusion speed of the molecules, i.e. a faster decay signifies a larger diffusion coefficient and vice versa. The absolute amplitude of an ACF depends on the fluorophore concentration. A larger concentration implies a larger average number of molecules within the detection volume, so that the amplitude of intensity fluctuations due to diffusing molecule entering or leaving the volume become smaller. Therefore, the ACF amplitude is inversely proportional to the concentration of fluorophores in solution, i.e. to the average number of fluorescent molecules in the detection volume. Thus, FCS can be utilized for both quantifying the diffusion and the absolute concentration of fluorescent molecules in a solution. On shorter time-scales (microseconds), the temporal behavior 

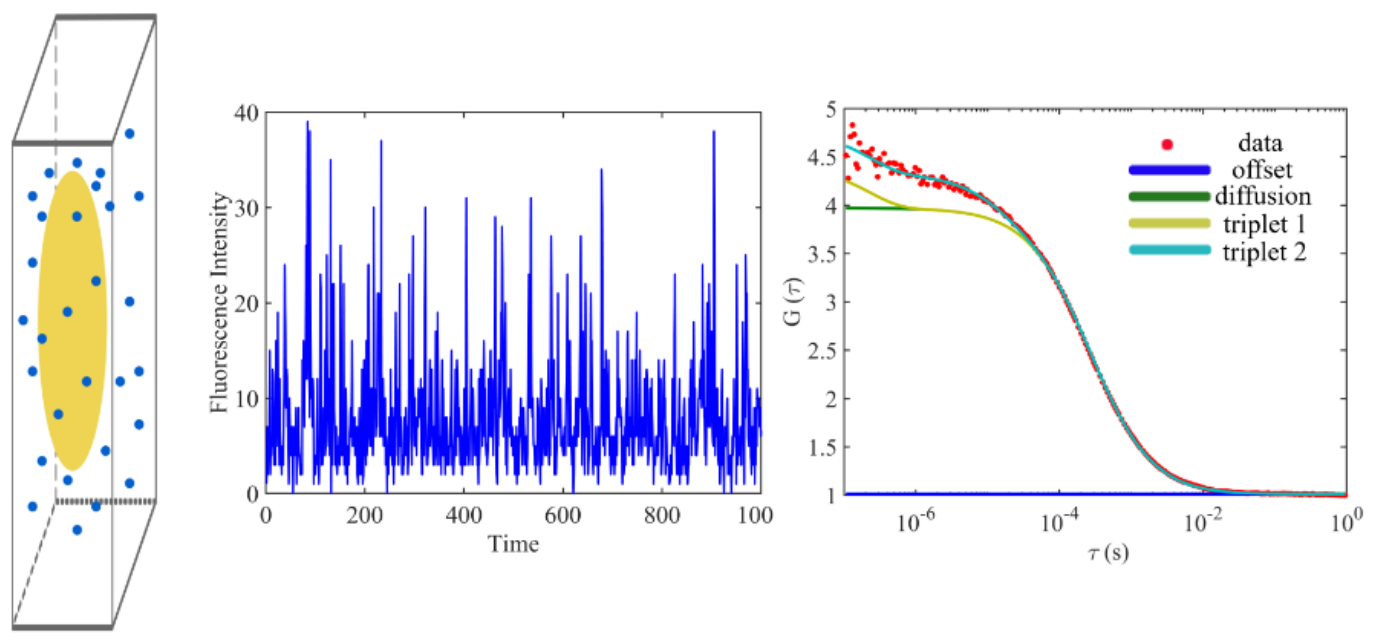

Figure 2.7: A typical FCS experiment. Fluorophores (blue circles in left panel) diffuse in and out of a small detection volume (yellow region in left box). Due to the constantly varying number of molecules within the detection volume, the recorded fluorescence intensity exhibits strong fluctuations (middle panel). The right hand graph demonstrates the anatomy of a typical autocorrelation function (ACF) measured on Atto 655 in aqueous solution. Data is shown by red circles, an offset which stems form physically uncorrelated photon pairs is shown in blue. The ACF decays due to translational diffusion of the dye molecules. A fit of this diffusion-related decay is shown as a green solid line. The yellow and cyan solid lines depict correlation decays due to triplet state photophysics (transitions from the fluorescent state to a non-fluorescent triplet state) on a time-scale of microseconds.

of an ACF can be influenced by other processes, such as molecular photophysics or fast conformational fluctuations (as in PET-FCS in flexible polymers). On the nanosecond timescale, the ACF is characterized by so-called photon anti-bunching. This photonantibunching, which is an anti-correlation at nanoseconds lag time, is due to the fact that a molecule can emit on average only one single photon during its fluorescence lifetime. This, for lag times approaching the fluorescence lifetime, one observes a characteristic anti-correlation with a lag-time behavior determined by the lifetime value. Finally, when performing FCS experiments with polarized excitation and/or detection, rotational diffusion of the fluorescent molecules will lead to non-trivial temporal correlations on time scales form nano- to microseconds, depending on the size of the fluorophores.

Due to its simplicity, FCS has found broad applications in physics, physical chemistry, and the life sciences, for instance for studying protein interactions [81], biomolecular conformational dynamics [82], cis-trans isomerizations [83], or chemical kinetics [84]. Excellent reviews on FCS can be found in refs. [85-90]. 
An advanced variant of the classical single-focus FCS was introduced in 2007, known as dual-focus FCS [91]. This method is used for precise and absolute measurements of diffusion coefficients. Unlike classical FCS, dual-focus FCS is insensitive to all kinds of optical aberrations such as caused by optical saturation, refractive index mismatch, cover-slip thickness variations, or optical imperfections of the used microscope [92-94]. Another important extension of classical FCS that should be mentioned here is dual-color fluorescence cross-correlation spectroscopy (FCCS). In FCCS, one records the fluorescence from two spectrally distinct fluorescent species in two detection channels, and then calculates not only the ACFs for each species, but also the cross-correlation between species. This can then be used to study protein-protein interactions, such as dimerizations, or enzyme kinetics [95-97]. In chapter 5, I present a publication where we have used both FCCS and dual-focus FCS to investigate the structure and dynamics of a protein.

In the next paragraph, I will detail another powerful extension of FCS, known as fluorescence lifetime correlation spectroscopy or FLCS. FLCS or lifetime-specific FCS is an integral part of this thesis, which I have utilized to investigate rapid conformational dynamics in an autofluorescent protein, which manifests itself by characteristic lifetime fluctuations.

\subsubsection{Fluorescence lifetime correlation spectroscopy (FLCS)}

FLCS is an extension of the core idea of FCS, but exploiting fluctuations of the fluorescence lifetime. It is similar to FCCS which looks at the correlation between two different spectral channels, but using different fluorescence lifetime states instead of different emission colors [37]. Thus, the core idea of FLCS is to perform cross-correlations between fluorescence signals having distinct lifetime rather than spectral signatures. In experimental terms, it is based on an upgrade of FCS with fluorescence lifetime measurements [98]. Fluorescence lifetime determination can be done by measuring the time it takes for the fluorescence intensity to reach $1 / e$ of its initial value $I_{0}$ at time $t=0$ after excitation with a short pulse of light. Mainly two experimental methods exist for determining the fluorescence lifetime, time-correlated single photon-counting (TCSPC) [99], and the phase fluorometry [100]. In the phase fluorometry, a sample is excited by

light with sinusoidally modulated intensity. The resulting fluorescence emission of the sample directly follows the excitation modulation (thus showing the same modulation frequency). Since the fluorescence lifetime has a finite value, the emission modulation is delayed in phase with respect to the the excitation modulation. Thus, from the phase delay one can directly calculate the value of the fluorescence lifetime. However, phase fluorometry is not suitable for fluorescence lifetime measurements at the single molecule 


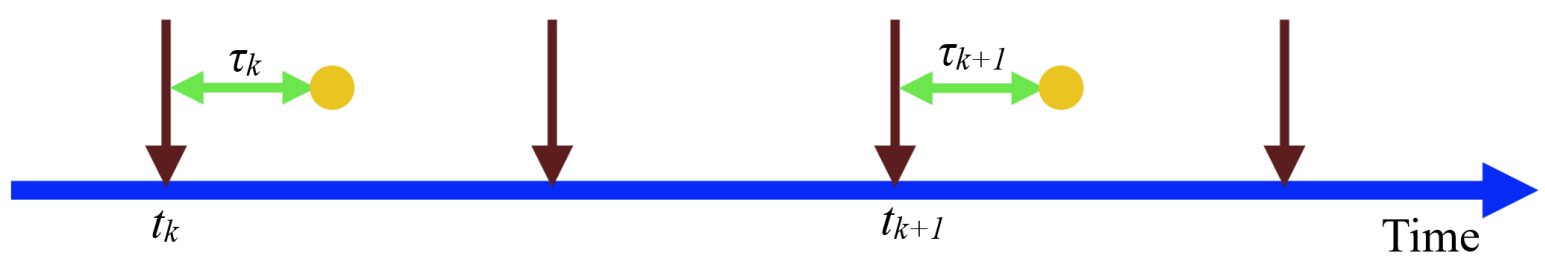

Figure 2.8: The figure visualizes the time-tagged time-resolved (TTTR) counting scheme. Timing of single-photon detection events is done on two different timescales. On a pico- to nanosecond time-scale, the detection time of each photon (yellow circle) is recorded with respect to the preceding laser pulse (so-called micro-time $\tau_{k}$ ). If one histograms these micro-times, a TCSPC decay histogram is obtained (shown in Figure 2.9, panel A) having a bin width determined by the discrete temporal resolution of the detection system. The macro-time $t_{k}$ records the absolute arrival times of photons on a time-scale with a temporal resolution equal to the laser pulse repetition period. From both time tags, the absolute detection time with an overall temporal resolution of picoseconds can be reconstructed.

level, for which TCSPC is the method of choice. TCSPC is a "stop-watch" method based on the ability of detecting and counting single photons. TCSPC was made possible by the emergence of single-photon sensitive detectors and the development of pulsed lasers. In TCSPC, a sample is excited with a high-repetition short-pulse laser, and the excited fluorescence is measured via single-photon counting (Geiger detection mode). Measuring the short delay times between excitation pulse and detected fluorescence photons require high-speed counting electronics with picosecond temporal resolution. By recording a large number of photon excitation-detection events, a TCSPC histogram is gradually recorded which presents statistics of detecting a photon with a given delay time after pulsed laser excitation. The exponential decay of this histogram over time then yields the fluorescence decay time. In TCSPC, each single photon detection are assigned two time tags, one is the micro-time $(\tau)$ and the other macro-time $(t)$. The micro-time has a temporal resolution of a few picoseconds, while the macro-time has a temporal resolution equal to the laser pulse repetition period. This mode of photon timing is called time-tagged time-resolved (TTTR) counting. Details of instrumentation and operation have can be found in refs. [98, 101]. A schematic of the TTTR counting mode is shown in Figure 2.8.

In practice, FLCS measurements are similar to classical FCS, involving one excitation source and one single detection volume. FLCS exploits the fact that two or more emitters in the same spectral range can be distinguished on the basis of their fluorescence lifetimes. In FLCS, one calculates auto-and cross-correlation functions by weighing recorded photons according to the their detection times on the nanosecond time scale after exciting 
A

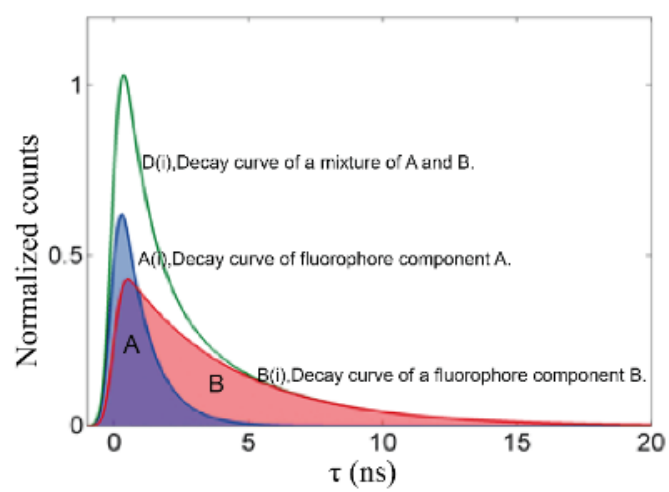

$\mathrm{C}$

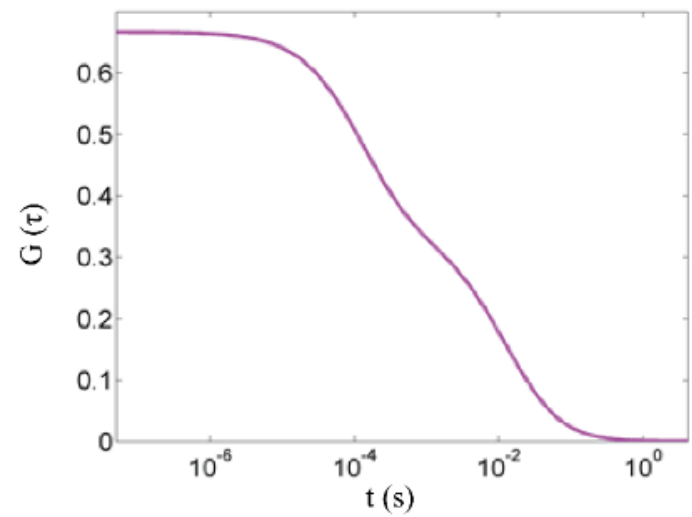

B

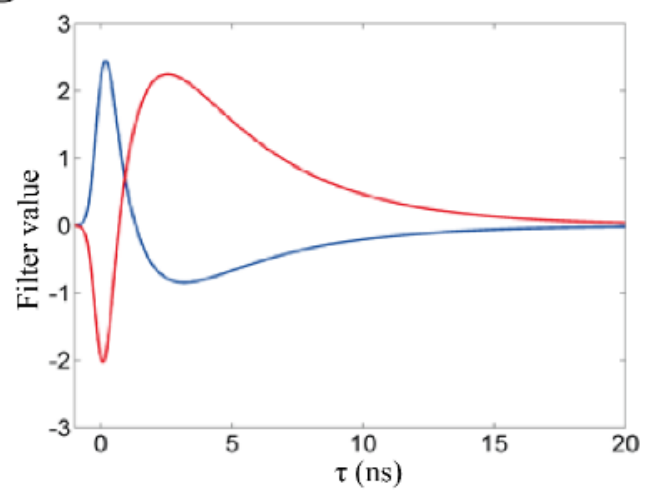

$\mathrm{D}$

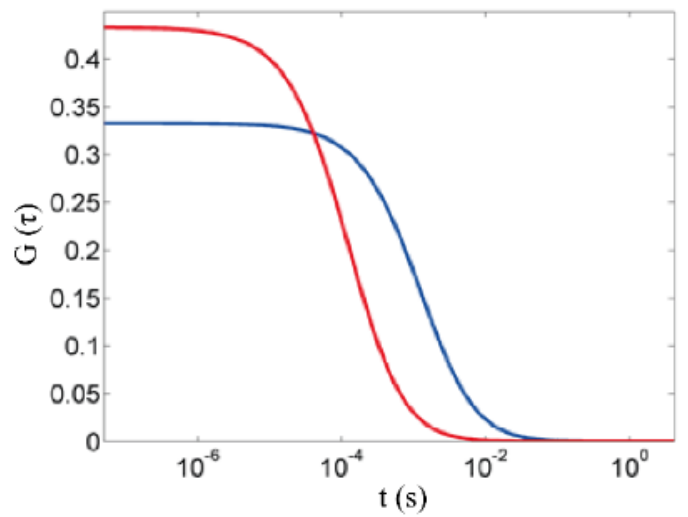

Figure 2.9: Working principle of FLCS. A. Total TCSPC curve of a mixture of species A and B (green), and individual TCSPC curves of each component A (blue) and B (red). B. Lifetime-specific filter functions calculated from the component-specific TCSPC histograms shown in A. Note that the filter function is positive for the shorter lifetime component for the first few nanoseconds, while it is positive for the longer component at longer decay times. C. ACF obtained with conventional FCS containing contributions from both the components A and B. D. Separate ACFs calculated for each component A and B by weighing the photons based on their arrival times with the filter functions shown in B.

with laser pulses (fluorescence lifetime timescale) [102, 103]. Figure 2.9 outlines the working principle of FLCS. FLCS has been utilized for investigating DNA compaction [104], proton transfer reactions[105], for membrane studies (in conjugation with STED) [106]. In my work, FLCS was a core technique that I have used as an advanced spectroscopic tool to quantify rapid photophysical transitions in the fluorescent protein EGFP [38]. A detailed account of the recent progress, novel applications, and extensions of FLCS together with the just mentioned study of EGFP is provided by my publications presented in the following chapter.

We have presented the utility of SMFS methods for quantifying fast processes down to nanoseconds regime with single molecule sensitivity. Next, we shift our focus to fluorescence microscopy, and super-resolution microscopy techniques in particular which are 
extensively used for discerning molecular architecture of biological structures with unprecedented details.

\subsection{Fluorescence microscopy}

Fluorescence microscopy allows to specifically visualize biological structures and molecules of interest. For this purpose, these structures and molecules have to be labeled with a fluorophore (an organic dye or a fluorescent protein). In principle, the target (a protein, DNA, cell membrane receptors etc.) is stained with a fluorescent molecule, mostly sitespecifically, and is then illuminated with light of a suitable wavelength for exciting the label fluorescence and this to visualize structures or to follow dynamical processes in time. In this context, it is important to note that the resolution of all conventional fluorescence imaging modalities is limited by the diffraction of light. As mentioned earlier, Ernst Karl Abbe was to first to state the fundamental limit of optical resolution in microscopy in 1873 [107]. He reported that the smallest possible distance $d$ where two point emitters can still be resolved by a microscopy is equal to half the wavelength $\lambda$ of the used light divided by the the refractive index $n$ of the sample medium and the half-angle $\theta$ of light collection of the employed objective, or

$$
d=\frac{\lambda}{2 n \sin \theta} .
$$

The product $n \sin \theta$ is called the numerical aperture (N.A.) of the the objective lens, and it can reach values up to 1.4 - 1.6 for most advanced objective designs and large values of $n$. In practice, the lateral resolution of a conventional optical microscope is $\sim 200 \mathrm{~nm}$. Two major classical microscopy modalities which are substantially utilized are wide-field and confocal microscopy.

Wide-field microscopy is widely used in bio-imaging. Non-fluorescent imaging methods where the wide-field illumination is utilized by detecting samples based on absorption and refractive index instead of fluorescence include bright-field [108] microscopy, differential interference contrast (DIC) microscopy [109], and Zernike phase-contrast microscopy [110]. In wide-field fluorescence microscopy, one uses a uniform illumination, but the sample is stained with fluorophores, so that the excited fluorescence emission provides the desired contrast for structures of interest [111]. The major drawback of wide-field fluorescence microscopy is that it collects light from all planes, so that structures from all positions along the optical axis contribute to the final image, leading to a strong background and preventing to obtain a three-dimensional image of a sample. A schematic of a wide-field microscope is shown in Figure 2.10. 
Confocal microscopy $[112,113]$ is a technique that was specifically invented to overcome the inability of a wide-field microscope to generate a three-dimensional image of a sample. The method uses a laser excitation source that is focused into a diffraction-limited spot into the sample, instead of illuminating the whole sample at once as done in wide-field microscopy. The emission light is collected by the same objective as used for focusing the laser light, separated from the excitation path by a dichroic mirror, and then focused through a confocal circular pinhole with diameters ranging from $\sim 25$ to $150 \mu \mathrm{m}$. After the pinhole, the light is finally focused onto a single-point detector (for example a photo-multiplier tube (PMT) or an avalanche photodiode (APD)). This so-called confocal detection rejects most of the light coming from positions below or above the focal plane, thus restricting detection to one specific plane of the sample. By scanning the excitation focus and co-moving detection in three dimensions over the sample, a threedimensional image of a sample can be acquired. Scanning can be done either using a galvoscanner for beam scanning, or by moving the whole sample through the stationary focus using a piezo-mechanical translation stage (see Figure 2.10). Contrary to wide-field illumination, in confocal microscopy the excitation laser beam is first expanded to overfill the back aperture of the objective, so that after the objective, the light is focused into a diffraction-limited spot. This focused illumination spot has diameters between 0.25 to $0.8 \mu \mathrm{m}$ (depending on the N.A. of the objective), and confocal detection typically extends over a depth range of 0.5 to $1.5 \mu \mathrm{m}$. As already stated, the major advantage of a confocal system is its ability to generate a three-dimensional image of a sample. Nowadays, confocal microscopy is the most widely used method for bio-imaging, especially in thick samples. Comprehensive information about confocal microscopy is given in refs. $[114,115]$.

Last but not least, I want to mention fluorescence lifetime imaging microscopy (FLIM). FLIM is a powerful extension of fluorescence microscopy [116-118] which allows not only to obtain fluorescence intensity images, but also to measure the fluorescence lifetime in a spatially resolved manner. In a FLIM image, one uses the fluorescence lifetime in a similar manner as emission color in a multi-color detection microscope. Since the fluorescence lifetime is independent of fluorophore concentration, absorption, thickness of sample, or photobleaching, FLIM is much more robust to variations in these parameters than intensity-based microscopy. 
Wide-field

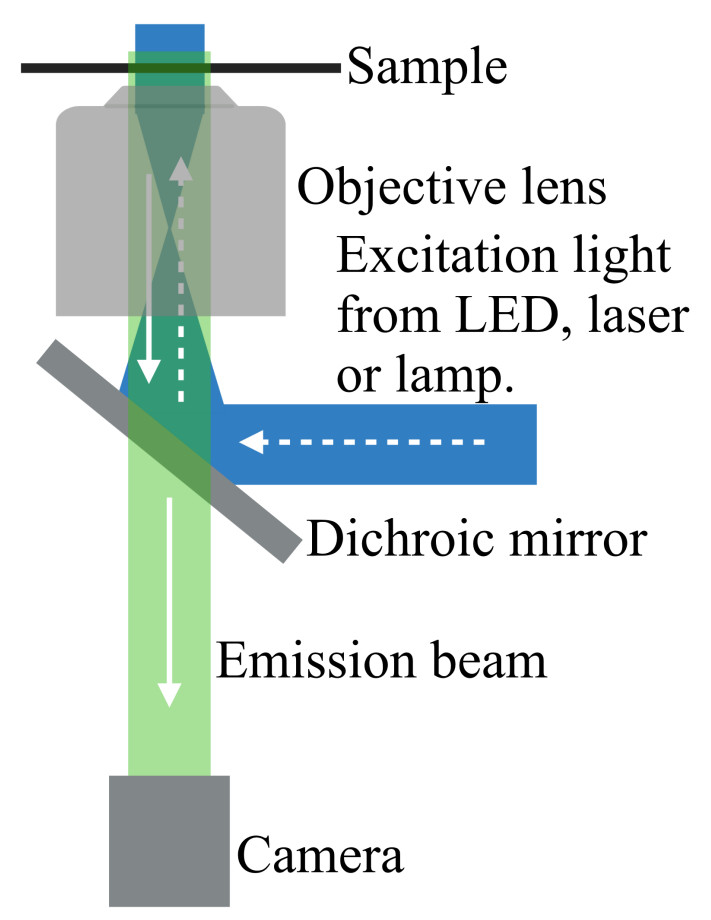

Confocal

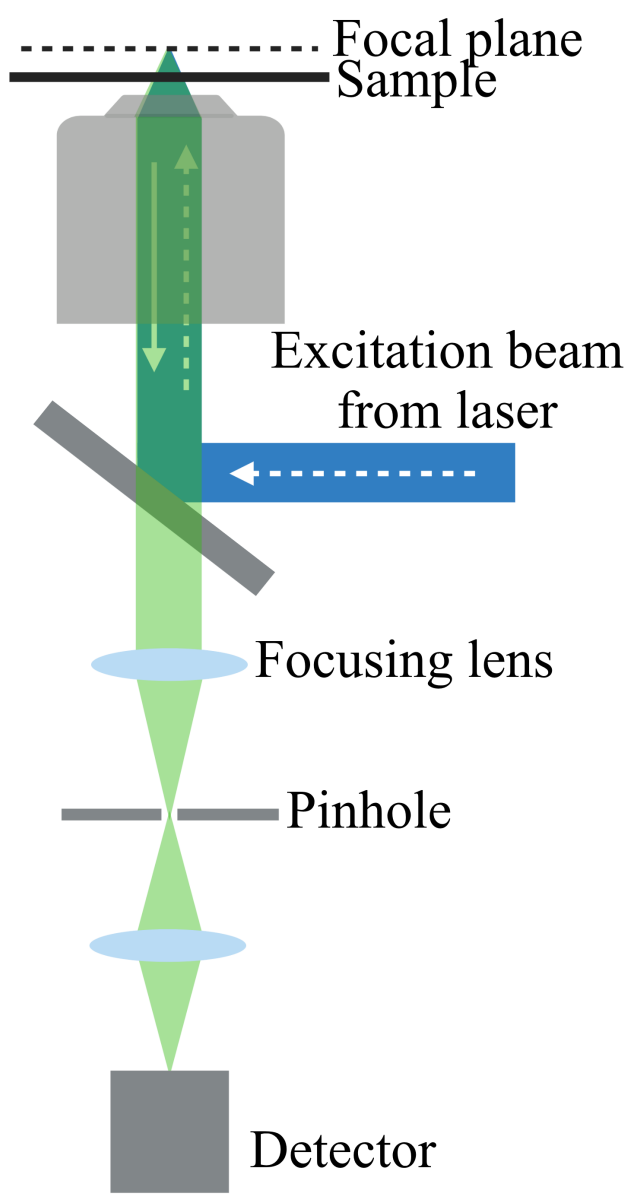

Figure 2.10: Left panel shows a wide-field epi-fluorescence microscope. Excitation light is shown in blue, and it is reflected by a dichroic mirror towards the objective lens for sample wide-field illumination. Fluorescence emission (shown in green) is collected by the same objective (epi-fluorescence setup) and focused onto a camera using a tube lens. The right panel visualizes a confocal microscope. In contrast to wide-field excitation and detection, here fluorescence is excited by a focused laser beam and collected fluorescence light is send through a confocal pinhole that rejects out-of-focus light, letting pass to the detector only emission from the focal plane. 


\subsection{Super-resolution fluorescence microscopy}

\subsubsection{Diffraction barrier of optical resolution}

The diffraction limit of optical resolution is due to the wave nature of light. When light from a single point source is imaged by a microscope onto the image plane, the resulting image is a spot of finite size, the diameter of which is determined by the interference of the converging light. This spot size determines the theoretical resolution limit of the imaging microscope. This image of an ideal point source is also called the point-spread function (PSF), and the image of an arbitrary sample is given by the convolution of this PSF with the distribution of fluorescent labels in the sample (weighted with the appropriate label fluorescence brightness) [119]. The shape of the PSF exhibits a characteristic ring structure surrounding a central peak, which is known as Airy pattern [120]. The size of this pattern, and thus the spatial resolution of the microscope, scales linearly with the wavelength of the used light, and inversely with the numerical aperture of the used objective. Thus, to increase the spatial resolution of imaging, one can either use light of shorter wavelengths, or an objective with larger N.A. But both options have some fundamental limits. An additional but more complex way to increase resolution is to combine non-uniform so-called structured illumination with wide-field detection. This is realized by structured illumination microscopy (SIM) [121] and image scanning microscopy (ISM) [122, 123], which both double the resolution of a conventional wide-field or confocal microscope.

A completely new way to increase the spatial resolution beyond the diffraction limit is provided by the recently modalities of super-resolution microscopy, which will be the topic the next section.

\subsubsection{Super-resolution imaging techniques}

The first real far-field super-resolution microscopy (in the sense of actually "breaking" the diffraction limit of resolution) was stimulated emission depletion or STED microscopy developed by S.W. Hell in 1994 [3]. As the name already says, STED microscopy is based on stimulated emission, i.e. the forced transition of an excited fluorescent molecule to its ground state by an external strong light source of suitable wavelength. The brilliant idea behind STED is to first bring fluorescent molecules into their excited state by a tightly focused laser spot (as done in conventional confocal microscopy), but then to de-excite some of them via stimulated emission with a donut-shaped intensity distribution around the central focus. This leaves only molecules in a very compressed region around the focus 
center in their excited state, and only these molecule then contribute to the fluorescence signal recorded at this focus position [3], see Figure 2.9. By scanning the sample with such a configuration, one obtains a scan image with a spatial resolution which is solely determined by the power of the stimulated-emission donut beam. Donut-shaped beams can be generated by appropriate phase masks.

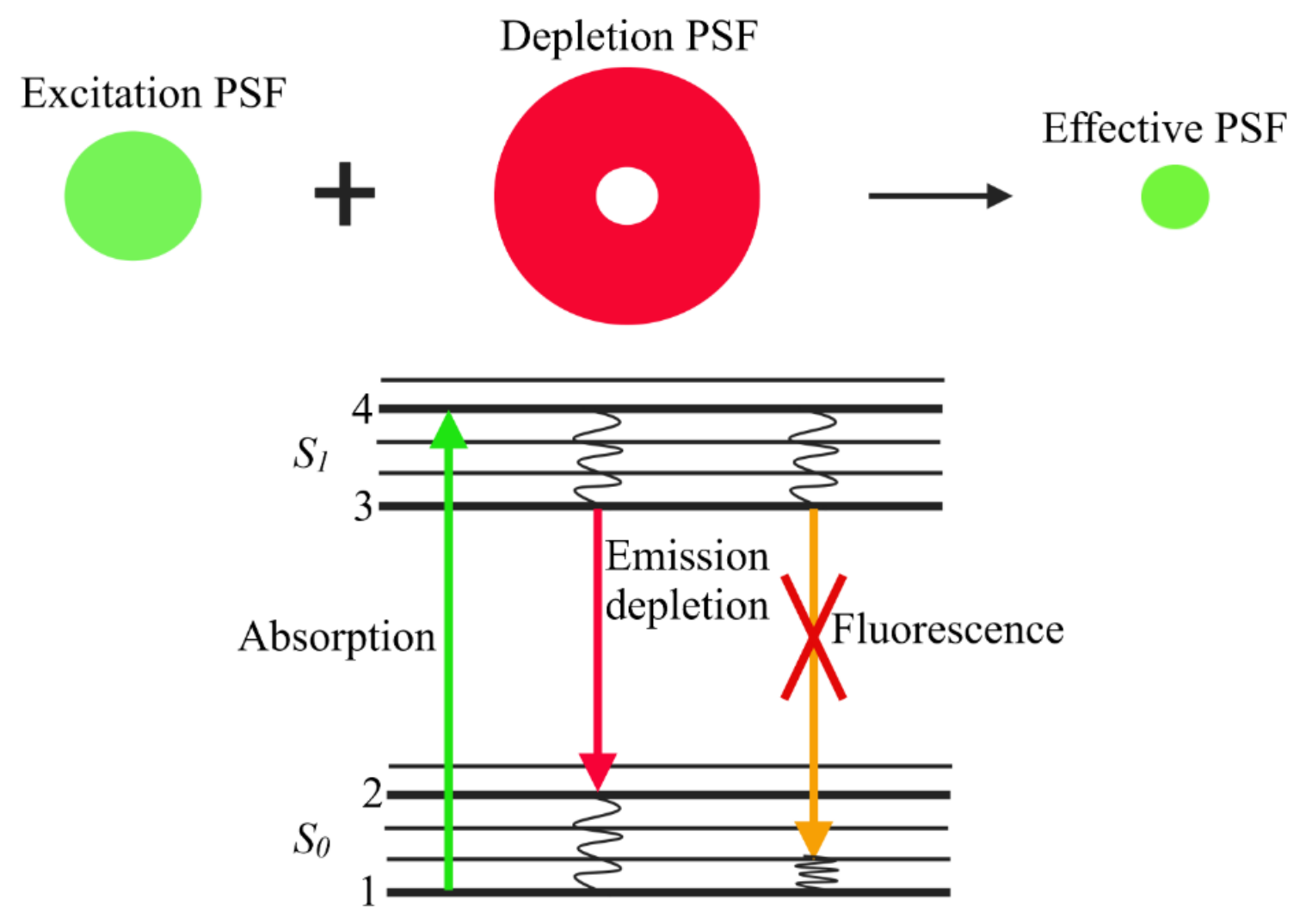

Figure 2.11: The underlying principle of STED microscopy. Top panel illustrates the effective point spread function (PSF) of a STED microscope. The excitation PSF (green) overlaps with a donut-shaped STED laser focus (red) which generates the effective PSF. The STED beam is used to deplete excited molecules in regions around the central excitation focus. A Jablonski scheme showing excitation together with spontaneous and stimulated emission is depicted in the panel below.

The achievable STED microscopy resolution is approximately given by

$$
d=\frac{\lambda}{2 n \sin \theta \sqrt{1+I / I_{\text {sat }}}}
$$

which is an extension of Abbe's equation. Here, $I$ is the peak intensity (photon flux per unit area) of the donut beam and $I_{s a t}=(\sigma \times \tau)^{-1}$ is the intensity at which a fraction of 
$1 / e$ of the excited molecules is forced down to their ground state, where $\sigma$ is the absorption cross-section for stimulated emission, and $\tau$ is the lifetime of the first singlet state. Although STED microscopy can be used with some photostable mutants of fluorescent proteins to achieve a resolution of $\sim 50 \mathrm{~nm}$, its major drawback is the requirement of very high illumination intensities for efficient stimulated emission, due to the short lifetime of the first excited singlet state of a few nanoseconds. These high excitation intensities are often photo-toxic for living cells, thus limiting the application of the method. Nevertheless, STED has been successfully implemented for video-rate imaging $(28 \mathrm{~Hz})$ in combination with fast laser scanners at small areas of synaptic vesicles [124]. Multiple applications of STED have been reported and many excellent reviews on the method can be found in scientific literature [106, 125-127]. A recent development by S.W. Hell and colleagues is MINFLUX [128, 129], where the position of a fluorophore is determined by asynchronously scanning a donut-shaped excitation beam over the molecule, which allows for its extremely precise localization ( $1 \mathrm{~nm}$ ) with minimal photon fluxes.

A completely alternative approach to super-resolution microscopy is represented by the large class of methods based on single molecule localization. It emerged over the last $\sim 15$ years and is comprised under the name single molecule localization microscopy (SMLM). It relies on switching of fluorophores between a dark and a fluorescent state. This switching is used to separate the fluorophores in time and then to localized them with high precision individually. By thus recording the super-resolved positions of ideally all fluorophores across a sample, a super-resolved image is finally generated. The general idea of SMLM is presented in Figure 2.10. Several variants of SMLM exist, and the first ones were photoactivated localization microscopy (PALM) [7] and stochastic optical reconstruction microscopy (STORM) [8] developed by E. Betzig and X. Zhuang, respectively. Later developments are fluorescence PALM (fPALM) [130], direct STORM (dSTORM) [9], or point accumulation for imaging in nanoscale topography (PAINT) [10, 131].

The achievable spatial resolution of these methods is determined by the accuracy of finding the center position of a single molecule's image, quantified by the standard error of the fitted position. This accuracy can be pushed towards ever smaller values by recording more photons and by reducing noise. In the ideal case of a background-free shot-noise limited measurement, and neglecting detector pixelation effects, the error $\Delta x$ of determining an emitter's position is given by

$$
\left\langle\left(\Delta x^{2}\right)\right\rangle=\frac{\sigma^{2}}{N}
$$

where $\sigma$ is the standard deviation of the point-spread function (PSF), and $N$ is the number of collected photons. 


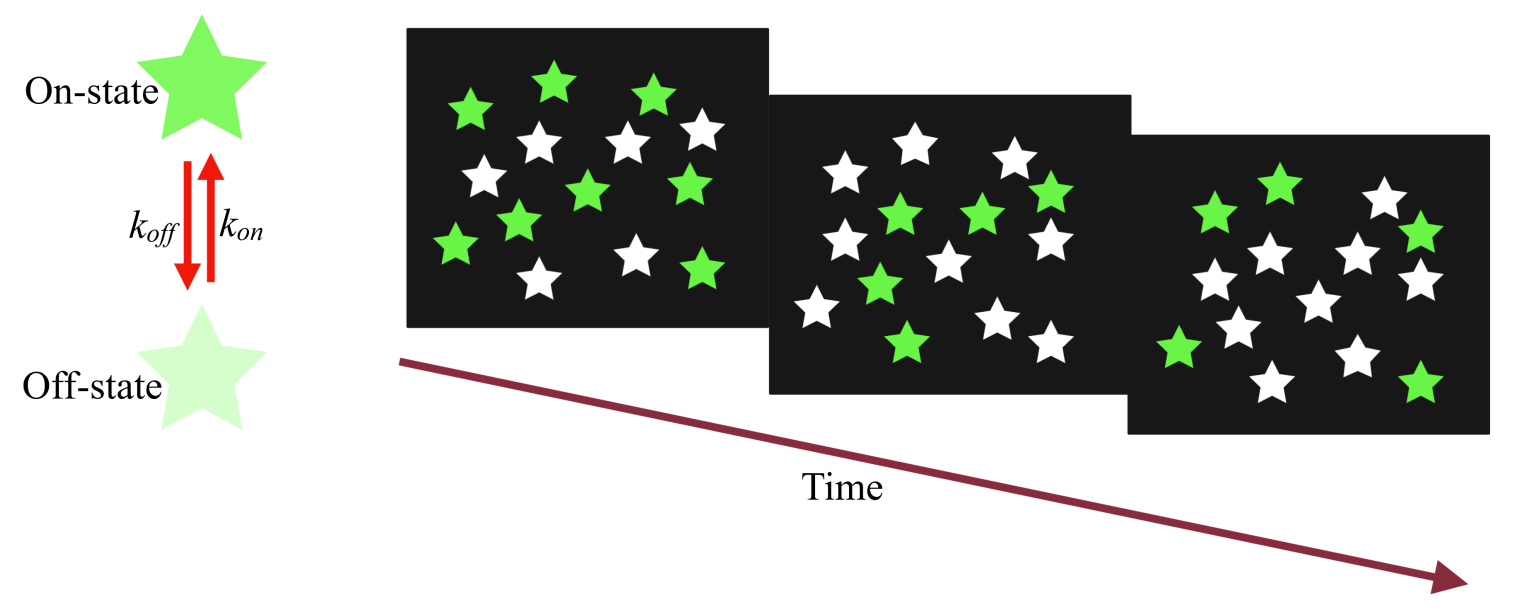

Figure 2.12: Principle of single molecule localization-based super-resolution microscopy. A fluorophore is switched between 'on' and 'off' states or irreversibly from 'off' to 'on' state. One records consecutive frames with different sub-set of molecules switched into a fluorescent on-state such that in each image the fluorescing molecules are spatially separated. In a subsequent step, images of individual molecules are identified and localized with high precision.

In PALM, a photo-activable fluorescent protein (PA-FP) is used for tagging structure(s) of interest. The experimental procedure is similar to the one described above, with the only difference that the fluorophores is not reversibly switched between bright and dark states but only switched on once and then ideally bleached in one step. Common examples of PA-FPs used for PALM are the fluorescent proteins PA-GFP, Dronpa, Kaede, EosFP, PA-mcherry etc. [7, 132, 133]. PALM finds its most applications in imaging of fixed samples, or for counting molecules in clusters and complexes [134]. Additionally, it is an useful tool for single-particle tracking experiments in living cells [135]. However, a drawback of PALM is the scarcity of photo-activable bright FPs, limiting its wide range of applications.

STORM is based on switching a fluorophore between 'on' and 'off' states in a controlled and reversible manner by using light of various wavelengths. The technique was first demonstrated by Xiaowei Zhuang and co-workers for Cy5-Cy3 dye pair which behaved as a photo-switching single molecule. In that case, a red laser was used for both fluorescence excitation and switching Cy5 into a dark state, while a green laser light was used to bring the dye back from its dark state to a fluorescent (this was assisted by the presence of the Cy3) [8]. STORM has been i.a. utilized for imaging telomeric loops [136], actin in axons [137], synapses [138] any many more. Both PALM and STORM achieve a typical lateral resolution of $\sim 20-25 \mathrm{~nm}$. Direct STORM or dSTORM was developed by Markus Sauer and colleagues $[9,139,140]$. The term 'direct' in dSTORM is used because the method does not require a second dye to be in vicinity of the fluorophore. The fluorophore is directly switched between fluorescent 'on' and 'off' states using laser light of 
suitable wavelength. Cyanine dyes (for example Cy5, Alexa fluor 647) are favorably used in dSTORM. Proof-of-concept dSTORM experiments were done by imaging actin filaments and microtubules, attaining a resolution of $21 \mathrm{~nm}$. Apart from cyanine derivatives with long-lived off-states, dSTORM has been extended for other standard organic fluorophores such as oxazine and rhodamine derivatives by stabilization of dark states using oxygen scavenging systems, because triplet states of such dyes can be utilized as off-states if oxygen is efficiently removed. As was known previously, oxazine and rhodamine dyes are quenched by electron donors such as the amino acid tryptophan. Following this idea, it was realized that thiol-containing reducing compounds such as b-mercaptoethylamine (MEA), dithiothreitol (DTT), or glutathione (GSH), that is substances with slightly lower redox potential than aromatic amines, can be effectively used to quench the triplet states of many Alexa Fluor and Atto dyes. The $\mathrm{pH}$ of the solvent governs the quenching efficiency since most thiols (-RSH) have a $\mathrm{pK}_{a, S H}$ of 8-9 and the reducing species is the thiolate anion (RS). The triplet state, having a longer lifetime than the first excited single state is even quenched at $\mathrm{pH} 7-8$, at lower RS concentrations. Efficient quenching of the triplet state under physiological conditions requires $10-100 \mathrm{mM}$ of thiol. dSTORM has found wide applications in super-resolution imaging of biological structures, see for example refs. [139, 141-143]. The most further development of dSTORM is its combination with lattice light-sheet microscopy for whole-cell imaging of plasma membrane receptors [144].

PAINT is based on the reversible binding of freely diffusing fluorophores to suitable functionalized target sites on structures of interest [10]. While diffusing in solution, fluorophores generate only a broad background in an recorded image but are not identified or localized as single molecules. As soon as they bind to an immobile target structure, they are discernible as bright immobile single molecules and can thus be localized in a similar way as is done in PALM or (d)STORM. Binding can be due to various interactions, such as hydrophobic, electrostatic etc. The binding and unbinding rates can be regulated via free fluorophore concentration and modulating binding affinity. An advanced variant of PAINT is DNA-PAINT, which relies upon the binding and unbinding of dye-tagged ('imager') oligonucleotides to complementary target ('docking') strands attached to a target of interest [131]. Due to the continuous replenishment of fluorophores, photobleaching problems as present in PAINT or dSTORM are circumvented and one can, in principle, record an unlimited number of single fluorophore localizations, leading to molecular-scale spatial resolution (sub $\sim 5 \mathrm{~nm}$ ). Furthermore, DNA-PAINT allows for principally unlimited multiplexing, and for molecule counting with high precision [145-147]. 


\subsection{Axial localization of fluorophores}

In classical laser-scanning confocal microscopy, the lateral resolution is ca. 2-5 times better than the axial resolution (along the optical axis). For example, the spatial resolution along the optical axis of a confocal microscope is on the order of $\sim 500 \mathrm{~nm}$ for light in the visible spectrum. Remarkably, this difference in lateral and axial resolution remains also for most of the above-mentioned super-resolution methods. For SMLM, the most popular approaches to localize single molecules also along the optical axis (and thus realizing true three-dimensional super-resolution microscopy) are astigmatic imaging [15], bi-plane imaging [16], and wavefront shaping [148].

Astigmatic imaging was one of the first methods for achieving three-dimensional superresolution in SMLM. For doing that, a cylindrical lens is placed in the imaging path for generating two focal planes differing slightly for the $x$ and $y$ directions [15, 149]. This results in a generally elliptic PSF, where its orientation and ellipticity code for a fluorophore's axial position along the optical axis. These raw images are fitted with a two-dimensional Gaussian to extract the $x$ and $y$ coordinates of the peak position as well as the principal axes of the elliptic PSF which determines a fluorophore's $z$ coordinate. 3D STORM imaging using astigmatism achieved a resolution of 50 to $60 \mathrm{~nm}$ along the optical axis while the lateral resolution was 20 to $30 \mathrm{~nm}$. As an altertnative to astigmatic imaging, bi-plane fPALM (BP fPALM) was introduced for three-dimensional imaging of samples having a depth of several micrometers [16]. In the bi-plane detection scheme, the emission light is divided in front of the camera using 50:50 beam splitter cube. The reflected light i.e. the longer path is redirected back towards the camera. The transmitted and reflected beams form images at different regions of the detector which correspond to object planes $350 \mathrm{~nm}$ away and closer to the objective lens than the actual object, respectively. In the data analysis workflow, fluorescence signal originating from two regions are combined into a 3D raw data stack which comprises two planes. Following this, each data set is fitted with an experimentally obtained 3D PSF consisting of pixels neighboring to each detected fluorophore molecule, hence determining its $x, y$ and $z$ coordinates. Using BP fPALM, axial resolutions of $75 \mathrm{~nm}$ and a lateral resolutions of $30 \mathrm{~nm}$ could be attained. As a third approach, wavefront shaping was developed by W.E. Moerner and colleagues. It exploits the so-called PSF engineering for realizing three-dimensional super-resolution fluorescence imaging [17]. Using a special phase mask in the conjugate Fourier plane of the detection channel, a double-helix PSF (DH-PSF) is generated [148]. Due to this DHPSF, a single fluorophore appears on the detector as two closely placed lobes. These lobes are fitted with two Gaussian functions. Localizations along $x$ and $y$ are obtained from the central point $(x, y)$ of two lobes while the $z$-position is determined via the angle of the axis connecting the lobes. In all these approaches, the axial localization accuracy remains 
2-5 times worse than the lateral localization accuracy. Some exceptions to this rule are interferometric methods such as interferometric PALM (iPALM) [18, 19] or isoSTED $[20,21]$, but their elevated technical complexity limits their broad application.

FRET can be also efficiently utilized for distance measurements. As described earlier, FRET relies on excited-state energy transfer from a donor fluorophore to an acceptor fluorophore. However, the nature of the dipole-dipole interaction limits the working range of FRET to ca. $10 \mathrm{~nm}$. In this range, FRET has been successfully utilized for quantification of intra-molecular distances and for investigating conformational dynamics in biomolecules $[26,28]$. However, FRET requires double labeling of a sample and cannot directly used for localizing a single object in space.

However, the physics of FRET (near-field coupling of an excited fluorophore to a nearby absorber) is closely realted to two other techniques that have been successfully used for axial super-resolution: variable angle total internal reflection microscopy (va-TIRFM) [150], and super-critical angle fluorescence (SAF) imaging [151-153]. Both these methods are based on near-field effects of the electromagnetic field.

In total internal reflection microscopy (TIRFM), one generates via total internal reflection (TIR) an exponentially decaying (with distance to a surface) light intensity above a glass surface which is used to excite fluorophores in close vicinity to this surface. The minimum incidence angle at which TIR occurs (TIR angle) can be found with Snell's law

$$
n_{1} \sin \theta_{1}=n_{2} \sin \theta_{2}
$$

where $n_{1}$ and $n_{2}$ are the refractive indices of the two materials on both side of a planar interface, and $\theta_{1}$ and $\theta_{2}$ are the incidence and refracted angles (with respect to the interface normal) of plane a wave moving through the interface. If one sets the angle of refraction $\theta_{2}$ (TIR), one obtains the critical TIR incidence angle $\theta_{c}$ as

$$
\theta_{c}=\arcsin \frac{n_{1}}{n_{2}}
$$

For a glass/water interface, the value of $\theta_{c}$ is $48.6^{\circ}$. For all incidence angles larger than $\theta_{c}$, light is entirely reflected back into the first medium (1), and the second medium (2), the light intensity falls off exponentially with the distance from the interface (evanescent field). The penetration depth of this evanescent field can be as large as a few hundred nanometers for incidence angles slightly above the critical TIR angle. Within this range, fluorophores can be efficiently excited by the evanescent light field, leading to a highly confined excitation zone close to the cover-slip surface. The major advantage of confining the excitation of fluorophores in this way is that one obtains a much higher signal-to-noise ratio as compared to classical wide-field illumination. 
Variable angle TIRFM (va-TIRFM) is based on exciting a fluorescently labeled sample using light at various incidence angles. Consequently, the distance over which the evanescent field decays is altered, hence also the fluorescence intensity. The underlying core idea is that one can use the ratio of the observed fluorescence intensities measured at different incidence angles of excitation light to calculate the distance of the fluorescent emitters from the surface. This can be done with nanometric localization precision. The method has found multiple applications especially for investigations of cellular topography [154], for quantifying the axial motion of secretory granules in the ventral side of living cells [155], and for measuring distances between a cellular membrane and a substrate at focal adhesion points [156].

Super-critical angle fluorescence or SAF imaging involves splitting of fluorescence emission light into two channels corresponding to light emitted above and below the so-called critical angle of fluorescence emission [153]. The core physical idea is that if an an emitter far away from above a glass surface is observed from the glass side, it does not emit any light into angles above the TIR angle. However, if the emitter comes close to the surface, near-field components of its dipole emission can couple into angles above this TIR angle, and the corresponding emission is called super-critical fluorescence. The ratio between the fluorescence intensities ("classical" and super-critical) of the two channels can be utilized to calculate the axial distance of the emitter from the surface. This is due to the fact that the SAF contribution continuously increases with decreasing distance of the fluorescent from the water-glass interface. SAF imaging has been successfully implemented both with wide-field and with confocal microscopes [157, 158].

Similar to the above mentioned methods, va-TIRFM and SAF imaging, metal-induced energy transfer (MIET) does also exploit near-field effects for axial localization of fluorescent emitters. The method was developed and introduced recently by our research group [22] for axial localization of fluorophores with nanometer accuracy. This method was then further developed and modified with using a graphene monolayer instead of a metal, allowing for axial resolutions down to sub-nanometers, which represents an almost tenfold resolution improvement over all other methods. This variant of MIET was introduced as "graphene-based metal-induced energy transfer" (GMIET) [24], but later renamed as graphene-induced energy transfer (GIET). I was predominantly involved in the development of GIET during most of the time of this thesis, and my thesis will mostly focus on GIET and MIET. In the following paragraphs, I will briefly introduce the basic concepts of MIET and GIET. 


\subsubsection{Metal-induced energy transfer (MIET)}

MIET imaging was developed as a spectroscopy-inspired method for nanometer-precise optical localization of fluorophores along the optical axis [22, 159]. MIET relies on the optical near-field mediated energy transfer from an optically excited fluorescent molecule (donor) to a thin planar metal film of $\sim 10-15 \mathrm{~nm}$ thickness (Figure 2.11). This energy transfer leads to a shortening of the excited state lifetime of the fluorophore which can be experimentally measured as a reduction of the fluorescence lifetime. Because the efficiency of the near-field mediated energy transfer is extremely distance-dependent (distance between fluorophore and metal layer), a measured lifetime value can be converted into a distance value. This is similar to Förster resonance energy transfer (FRET) where the energy transfer happens between a donor and an acceptor molecule. However, in contrast to FRET, the interaction range of MIET, i.e. the range over which one can efficiently measure an energy transfer and thus localize a molecule is more than 10 times larger (150-200 nm). For converting a measured fluorescence lifetime value into an ax-
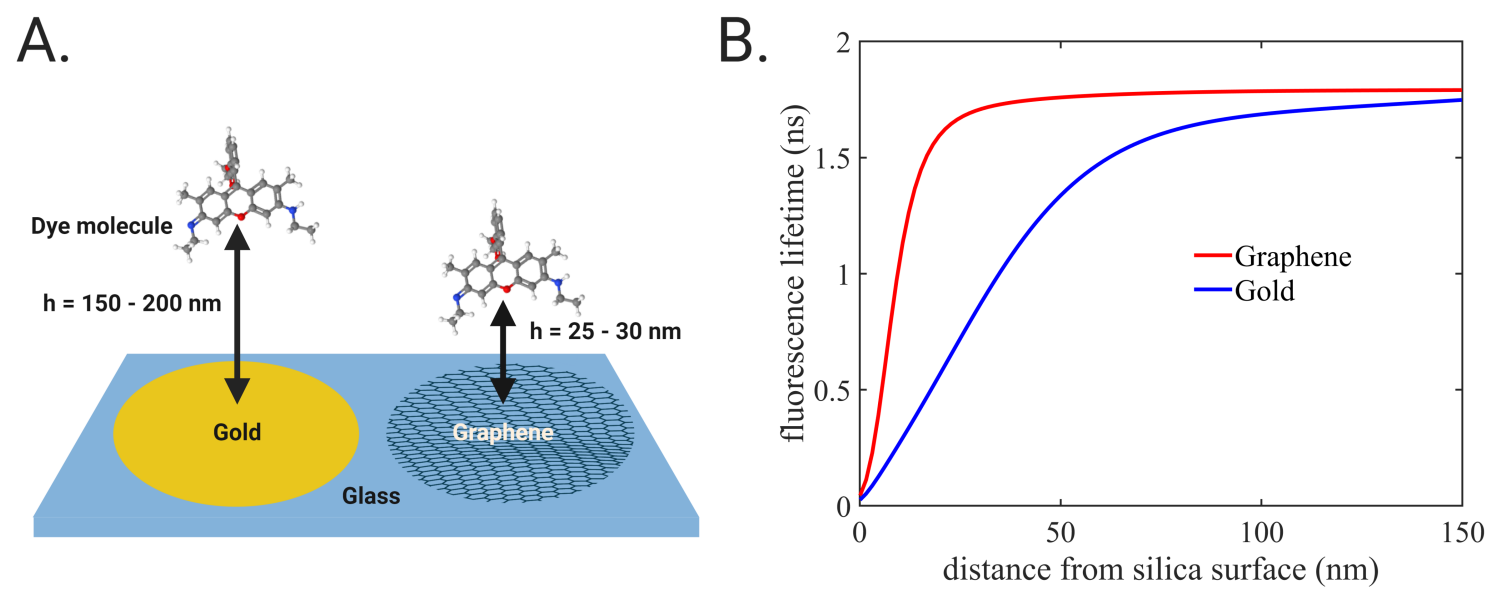

Figure 2.13: Working principle of MIET and GIET. A. The substrate consists of titan/gold/titan multilayer or a single graphene sheet on a glass coverslip, covered by a protecting silica layer (not shown). A fluorescent molecule within a range of up to ca. 150-200 nm (for metal) or 25-30 nm (for graphene) can electromagnetically couple to the metal or graphene layer which leads to a distance-dependent quenching of its fluorescence. B. Fluorescence lifetime as a function of distance of a molecule from the surface of a silica spacer ( $5 \mathrm{~nm}$ thickness) deposited on gold (blue) or graphene (red) layer. Calculations were performed for an emission wavelength of $680 \mathrm{~nm}$. The refractive index of the upper medium was set to 1.33 (water), and the refractive index of glass was set to 1.52 .

ial distance value, one needs to calculate a priori a calibration curve which is done by solving Maxwell's equation for the electric dipole emitter (fluorophore) above the metal or graphene covered glass substrate [160]. The achievable localization accuracy depends on the accuracy of the lifetime determination which, in turn, is inversely related to the 
square root of the number of photons recorded (similar to the localization accuracy in SMLM). The first application of MIET for biological imaging was the mapping of the basal membrane of living cells with nanometer accuracy [22]. For this purpose, three candidate cell lines were chosen: MDA-MB-231 human mammary gland adenocarcinoma cells and A549 human lung carcinoma cells, both used as metastasis in vivo models, and MDCK-II from canine kidney tissue as a benign epithelial cell line. In this work, significant differences in cell-substrate distance were observed between a normal epithelial cell and cancerous cell lines. MIET was also utilized to monitor cell-interface distance of NMuMG cells during epithelial-to-mesenchymal transition (EMT) [161]. Following this, dual-color MIET was developed and used to reconstruct the three-dimensional profile of the nuclear envelope over the complete basal area of HeLa cells [162]. In this work, axial distances between two representative proteins, Lap2 $\beta$ and Nup358, present in the nuclear envelope and the nuclear pore complex, respectively, were measured. Dual-color MIET was also combined with FRET for looking at adhesion points and cytoskeletal elements in human mesenchymal cells. While MIET was used to resolve nanometric structural details along the optical axis, FRET was employed for quantifying distances between actin and vinculin at focal adhesions [163]. At the single molecule level, MIET allowed localizations of immobilized dye molecules deposited on dielectric spacers of known thickness with an axial resolution of $\sim 2.5 \mathrm{~nm}$ [159]. Single molecule MIET (smMIET) was extended for axial co-localization of two dye molecules on a DNA origami pillar [164].

\subsubsection{Graphene-induced energy transfer (GIET)}

A further boost in axial resolution was achieved with the development of GIET where the metal layer of MIET is replaced with a single sheet of graphene. This allowed for improving the axial resolution by nearly ten-fold [24]. The price to pay is the ten-fold reduced working range of GIET which is $\sim 25 \mathrm{~nm}$ from the surface of the graphene (Figure 2.11). GIET was shown to be able to localize single emitters with nanometer accuracy, and to measure inter-leaflet distances in supported lipid bilayers (SLBs). To my knowledge, this cannot be easily done with other existing fluorescence nanoscopy techniques. A detailed overview of GIET, its theory, development and experimental applications can be found in the publications presented in chapter 4 . 


\section{Chapter 3}

\section{Fluorescence Lifetime Correlation Spectroscopy (FLCS)}

\subsection{Manuscript: Quantifying microsecond transition times using fluorescence lifetime correlation spec- troscopy}

Autofluorescent proteins represent a class of fluorescent emitters which frequently manifest more than one emission state leading to rapid fluctuations in their excited-state lifetime. In this manuscript [38], we quantify microsecond transition rates between two fluorescent states of EGFP, which have otherwise overlapping spectra. These fast transitions can be related to an angstrom-scale rotational isomerism of an amino acid residue next to the chromophore center. With this study, we demonstrate the power of FLCS for probing photophysical dynamics of complex luminescent systems which cannot be easily done with other existing methods.

The original research article entitled "Arindam Ghosh, Sebastian Isbaner, Manoel Veiga-Gutiérrez, Ingo Gregor, Jörg Enderlein, and Narain Karedla. Quantifying microsecond transition times using fluorescence lifetime correlation spectroscopy. The journal of physical chemistry letters, 8(24):6022-6028, 2017 " was published. Arindam Ghosh (A.G.) performed all FLCS experiments, co-wrote the manuscript and assisted in data analysis.

Reproduced with permission, copyright 2017 American Chemical Society.

The published research article is currently available at https://pubs.acs.org/doi/10. 1021/acs. jpclett. 7 b02707. 


\title{
Quantifying Microsecond Transition Times Using Fluorescence Lifetime Correlation Spectroscopy
}

\author{
Arindam Ghosh, ${ }^{\dagger}$ Sebastian Isbaner, ${ }^{\dagger}$ Manoel Veiga-Gutiérrez, ${ }^{\ddagger}$ Ingo Gregor, ${ }^{\dagger}{ }^{\circ}$ Jörg Enderlein, ${ }^{\dagger}{ }^{\oplus}$ \\ and Narain Karedla*, ${ }^{\dagger}$
}

\author{
${ }^{\dagger}$ III. Institute of Physics, Georg August University, 37077 Göttingen, Germany \\ ${ }^{\ddagger}$ PicoQuant GmbH, 12489 Berlin, Germany
}

Supporting Information

\begin{abstract}
Many complex luminescent emitters such as fluorescent proteins exhibit multiple emitting states that result in rapid fluctuations of their excited-state lifetime. Here, we apply fluorescence lifetime correlation spectroscopy (FLCS) to resolve the photophysical state dynamics of the prototypical fluorescence protein enhanced green fluorescent protein (EGFP). We quantify the microsecond transition rates between its two fluorescent states, which have otherwise highly overlapping emission spectra. We relate these transitions to a roomtemperature angstrom-scale rotational isomerism of an amino acid next to its fluorescent center. With this study, we demonstrate the power of FLCS for studying the rapid transition dynamics of a broad range of light-emitting systems with complex multistate photophysics, which cannot be easily done by other methods.
\end{abstract}

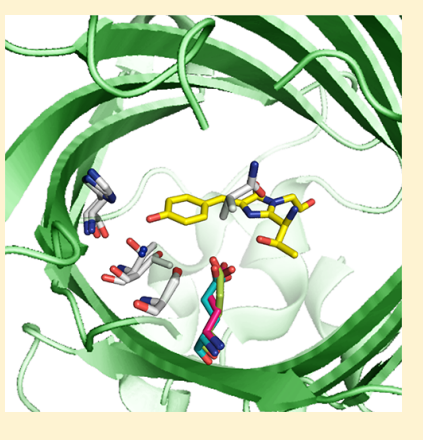

S ingle-molecule spectroscopy has developed into an experimental cornerstone for investigating dynamic conformational fluctuations and nanoscale sensing, in particular nanomechanical motions. ${ }^{1-7}$ The core basis of these applications is the exceptional sensitivity of many photophysical properties of single molecules, such as fluorescence intensity, excitation/emission spectra, and excited-state lifetime $\left(\tau_{\mathrm{fl}} \approx\right.$ $\left.10^{-9} \mathrm{~s}\right)$, to their local environment. Most widely used approaches include resolving spectral heterogeneity and intensity fluctuations of fluorescent probe molecules at cryogenic temperatures in condensed matter. ${ }^{8-12}$ However, at room temperature, thermally induced broadening of spectral lines often masks the impact of local environment fluctuations on single-molecule spectra. In such cases, measuring the excited-state lifetime can provide an alternative approach. In particular, the excited-state lifetime of a fluorescent emitter is determined by (i) the radiative dipole emission that depends on the local electric field; (ii) nonradiative transitions due to vibrational couplings to and collisions with surrounding molecules; and (iii) near-field coupling of the radiating dipole to surface plasmons or another dipole emitter (Förster resonance energy transfer). Thus, measuring fluorescence lifetime fluctuations can provide deep information about the local environment around a fluorescent molecule and changes thereof.

In most single-molecule experiments, the fluorescence lifetime is measured by time-correlated single-photon counting (TCSPC). In TCSPC, a molecule is repetitively excited by a train of short laser pulses, and the time delays between emitted photons and the exciting pulses are measured and histogrammed. However, typical single-molecule photon detection rates are on the order of $\sim 10^{3}$ to $\sim 10^{4} \mathrm{~Hz}$. This severely limits the temporal resolution of measuring lifetime fluctuations. ${ }^{13}$ In this Letter, we explore the potential of fluorescence lifetime correlation spectroscopy (FLCS) to resolve submicrosecond temporal fluctuations of fluorescence lifetimes at the singlemolecule level. The core principle of FLCS is a statistical unmixing of the fluorescence signal from a mixture of molecular species with different fluorescence lifetimes. ${ }^{14}$ So far, FLCS has been successfully applied to separate intensity fluctuations from a mixture of dye species with different lifetimes. ${ }^{15-18}$ In a similar vein, it was used for separating populations of the same dye that show different lifetimes for different locations. ${ }^{19,20}$ Another technical application was separating detector afterpulsing and background scattering from the actual fluorescence of a dye solution. ${ }^{21,22}$ Here, we apply FLCS for monitoring rapid conformational isomerization in the prototypical enhanced green fluorescent protein (EGFP).

In a fluorescent protein, the chromophore is tightly enclosed by an ordered arrangement of amino acids and water molecules. Partial charges on the surrounding atoms influence the chromophore through noncovalent interactions such as hydrogen bonding, $\pi-\pi$ stacking, or electrostatic repulsions. ${ }^{23-28}$ Therefore, rotations/rearrangements of neighboring amino acids will modulate the photophysical properties of the chromophore. Multiple amino acid conformations close to the chromophore have been observed in X-ray crystal structures for several fluorescent proteins. ${ }^{29-32}$ At room temperature, such conformational fluctuations might not be resolvable from their spectra. To date, only a few studies have been performed

Received: October 12, 2017

Accepted: November 28, 2017

Published: November 28, 2017 


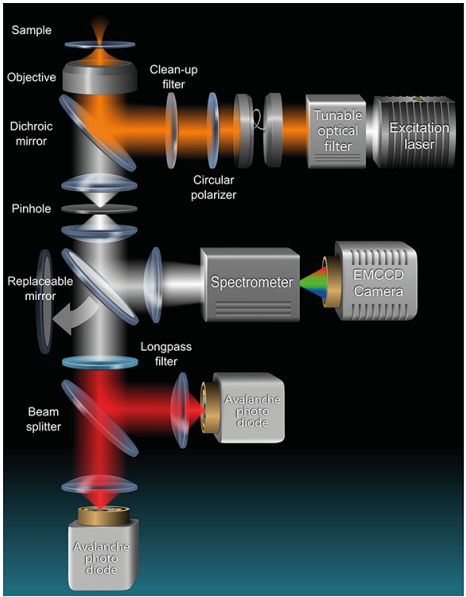

(a)

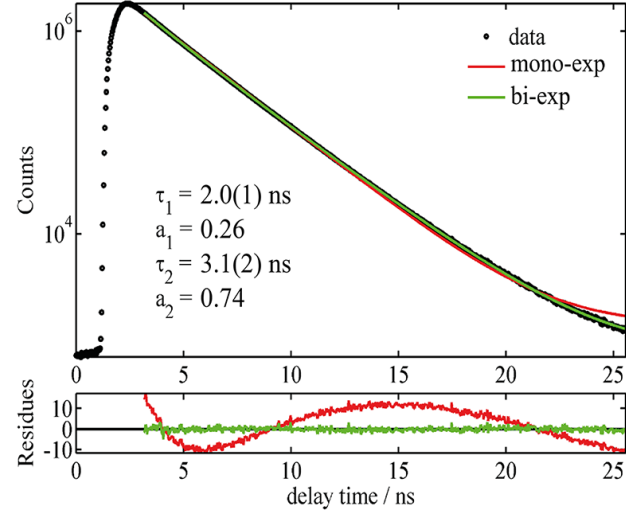

(b)

Figure 1. (a) Scheme of the setup used in FLCS measurements. (b) TCSPC data showing the histogram of arrival times of photons with respect to the excitation pulses. The data were fitted using monoexponential (red) and biexponential decay models. As can be clearly seen from the residues, the data can only be explained using a biexponential model. The two decay constants obtained from the fitting are 2.0(1) and 3.1(2) ns, with amplitudes of 0.26 and 0.74 , respectively.

at low temperatures using hole-burning spectroscopy to resolve the spectral shifts due to such conformational changes. 33,34 However, at room temperature, the presence of multiexponential fluorescence decays in various intrinsically fluorescent proteins such as citrine, CFP, ECFP, and dsRED has been reported in several articles. ${ }^{32,35-37}$ These studies attribute the multiexponential decay to local charge density fluctuations in the close vicinity of the chromophore, to rearrangements of putative hydrogen bonds, or to the interaction of an ATP molecule via an amino acid with the chromophore. Our work now focuses on the dynamic transitions between different lifetime states, which allows us to reconstruct the transition rate landscape of the conformational fluctuations around the chromophore of EGFP.

Briefly, a nanomolar solution of EGFP was excited, in a confocal microscope, with a pulsed diode laser $\left(\lambda_{\text {exc }}=485 \mathrm{~nm}\right.$, pulse duration $\approx 50 \mathrm{ps}$ fwhm, repetition rate $=40 \mathrm{MHz}$ ). The laser was passed through a cleanup filter (BrightLine FF01488/10, Semrock) and quarter-wave plate (AQWP05M-600, Thorlabs) to achieve a circularly polarized light, as shown in Figure 1a. The excited fluorescence light was split using a 50:50 nonpolarizing beamsplitter and detected with two singlephoton avalanche diodes. Details of the experimental setup of our confocal single-molecule spectrometer are described elsewhere. ${ }^{38}$ The observed fluorescence decay of EGFP can be well fitted with a biexponential decay model (Figure $1 \mathrm{~b}$ ) with decay constants of 2.0(1) and 3.1(2) ns. The two monoexponential components of this biexponential decay were next used to obtain the filter functions for calculating lifetimespecific fluorescence correlation curves. This yields two autocorrelation curves (one for each lifetime) and two crosscorrelation curves (transitions between the two lifetime states), as shown in Figure 2. The correlations were performed on a semilogarithmic time scale with the smallest time bin equal to the interpulse time distance of the laser $(25 \mathrm{~ns})$. The anticorrelation seen in both cross-correlation curves on the time scale of $10^{-7}-10^{-6} \mathrm{~s}$ corresponds to microsecond reversible switching between the two bright states. We denote these two states as $\mathbf{1}$ and $\mathbf{2}$, corresponding to the identified lifetime values of 2.0(1) and 3.1(2) ns, respectively. The decay

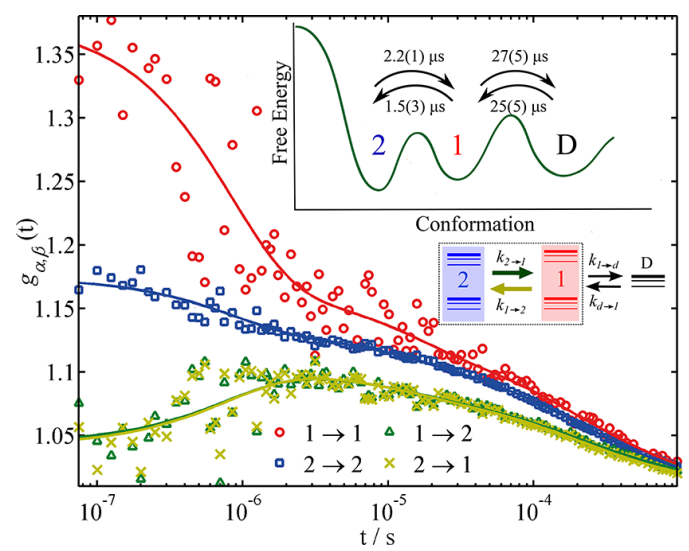

Figure 2. Calculated auto- and cross-correlations for EGFP solution measurements at $\mathrm{pH} 6.0$ with an excitation power of $\sim 200 \mathrm{~kW} \mathrm{~cm}^{-2}$. The $\mathbf{1} \rightarrow \mathbf{1}$ and $\mathbf{2} \rightarrow \mathbf{2}$ denote the autocorrelations of states $\mathbf{1}$ and $\mathbf{2}$, with $\tau_{1}=2.0(1)$ ns and $\tau_{2}=3.1(2)$ ns, respectively, whereas $\mathbf{1} \rightarrow \mathbf{2}$ and $2 \rightarrow \mathbf{1}$ represent the cross-correlations between the two species. The curves are normalized with respect to their amplitudes at time $0.1 \mathrm{~s}$ when all of the correlations have decayed completely. The fits using a three-state model are shown as solid lines. The half-time of each transition is listed in Table 1 . Note here that the correlation amplitude at $t \approx 0$ is less than the mean of the correlation amplitudes of the lifetime autocorrelations $\mathbf{1} \rightarrow \mathbf{1}$ and $\mathbf{2} \rightarrow \mathbf{2}$. This is mainly due to elimination of the contributions from background photons. The top inset shows the schematic free-energy landscape and relevant conformational switching between the three states, and the bottom inset represents the three-state reaction model and the rates involved.

of the correlation curves on a slower time scale between $10^{-6}$ and $10^{-4} \mathrm{~s}$ reveals a reversible transition to a dark state $\mathbf{D}$. At even longer time scales, the correlation curves decay due to the translational diffusion of EGFP molecules in and out of the excitation focus.

The curves were fitted with two-photon correlation functions $g_{\alpha \beta}$ derived from a three-state consecutive reaction model (see the inset of Figure 2), where $\alpha$ and $\beta$ take either the value of 1 or 2, corresponding to the two states $\mathbf{1}$ and $\mathbf{2}$ (see Supporting Information section S.3). The fast part (time scale shorter than 
the diffusion time of the molecule, $t \leq 10^{-4} \mathrm{~s}$ ) of the auto- and cross-correlation functions includes the rates of conversion between the two fluorescent states, denoted by $k_{1 \rightarrow 2}$ and $k_{2 \rightarrow 1}$, and the transition rates between states $\mathbf{1}$ and $\mathbf{D}$, denoted by $k_{1 \rightarrow \mathrm{d}}, k_{\mathrm{d} \rightarrow 1}$; see the inset of Figure 2. The rationale for a threestate consecutive reversible reaction model will be addressed later. The resulting correlation fit function is given by

$$
g_{\alpha \beta}^{\text {fast }}(t)=\kappa_{\alpha} \kappa_{\beta} \sum_{j=1}^{3} \hat{e}_{j, \alpha} \hat{e}_{j, \beta} \exp \left(-\lambda_{j} t\right)
$$

where $\kappa_{\alpha}$ and $\kappa_{\beta}$ are coefficients taking into account the relative brightness of the two fluorescent states and $\hat{e}_{j}$ and $\lambda_{j}$ are the eigenvectors and eigenvalues, respectively, of the rate matrix $\hat{T}$

$$
\hat{\boldsymbol{T}}=\left(\begin{array}{ccc}
-k_{\mathrm{d} \rightarrow 1} & k_{1 \rightarrow \mathrm{d}} & 0 \\
k_{\mathrm{d} \rightarrow 1} & -k_{1 \rightarrow 2}-k_{1 \rightarrow \mathrm{d}} & k_{2 \rightarrow 1} \\
0 & k_{1 \rightarrow 2} & -k_{2 \rightarrow 1}
\end{array}\right)
$$

The obtained means and standard deviations of the rate constants are $k_{1 \rightarrow \mathrm{d}}=4.3(8) \times 10^{4} \mathrm{~s}^{-1} ; k_{\mathrm{d} \rightarrow 1}=3.8(6) \times 10^{4} \mathrm{~s}^{-1}$; $k_{1 \rightarrow 2}=6.7(13) \times 10^{5} \mathrm{~s}^{-1}$; and $k_{2 \rightarrow 1}=4.5(2) \times 10^{5} \mathrm{~s}^{-1}$. The results show that the transition between the two lifetime states is an order of magnitude faster than the interconversion between states $\mathbf{1}$ and $\mathbf{D}$ (see Table 1). We repeated the FLCS

\section{Table 1. Average Switching Half-Times}

\begin{tabular}{cc} 
transition & switching time $(\mu \mathrm{s})$ \\
$\mathbf{1} \rightarrow \mathrm{D}$ & $25(5)$ \\
$\mathbf{D} \rightarrow \mathbf{1}$ & $27(5)$ \\
$\mathbf{1} \rightarrow \mathbf{2}$ & $1.5(3)$ \\
$\mathbf{2} \rightarrow \mathbf{1}$ & $2.2(1)$ \\
\hline
\end{tabular}

measurements for $\mathrm{pH}$ values in the range between 6.0 and 9.0 and for excitation powers from $\sim 4$ to $\sim 200 \mathrm{~kW} \mathrm{~cm}$ c $^{-2}$ (see, for example, Supporting Information Figure S4). The fitting results show that the transition rates between the two bright states are independent of these parameters, which rules out the possibility that any photoisomerization (at $485 \mathrm{~nm}$ excitation) or protonation/deprotonation of neighboring residues (if present) affects the bright states of the chromophore. From the ratio of the rates $k_{1 \rightarrow 2} / k_{2 \rightarrow 1}$, we obtain the state occupancy values of $\mathbf{1}$ and 2, which are $40(5)$ and $60(11) \%$, respectively.

Next, we characterized the excitation and emission spectra of the two states based on time-resolved fluorescence experiments. Spectrally resolved fluorescence decay curves were fitted globally with a biexponential decay model. Figure 3 shows the decay curves measured and the fits. The amplitudes of the two lifetime components were obtained as a function of the emission wavelength, as shown in Figure 3. The spectra of the two states show two peculiarities: (i) The emission peak of $\mathbf{2}$ is slightly red-shifted with respect to the peak of 1 , with the two emission maxima at 510 and $508 \mathrm{~nm}$, respectively. (ii) The emission spectrum of state $\mathbf{2}$ is broader than that of state $\mathbf{1}$. By integrating the amplitudes of the two spectra, we determined the relative intensities of the two states as 0.36(5) and 0.64(5), respectively, as shown in Table 2.

\section{Table 2. Spectral Properties of the Two States}

\begin{tabular}{ccccc} 
state & $\tau_{\mathrm{fl}}(\mathrm{ns})$ & rel. amp. & $\lambda_{\text {em }}(\mathrm{nm})$ & $\lambda_{\text {exc }}(\mathrm{nm})$ \\
$\mathbf{1}$ & $2.0(1)$ & $0.36(5)$ & $508.8(2)$ & $486.0(4)$ \\
$\mathbf{2}$ & $3.1(2)$ & $0.64(5)$ & $510.3(2)$ & $489.7(2)$ \\
\hline
\end{tabular}

Normalized excitation spectra (Figure 3) were obtained from the amplitudes of biexponential fits of excitation wavelengthspecific fluorescence decays. The amplitudes were normalized with the overall excitation spectrum to account for different excitation intensity and in such a way that the maximum of the sum spectrum was equal to 1 . The ratio of the area under the two spectra yields the intensity contributions of the two states, which are 39(7) and 61(7)\% for 1 and 2, respectively. As can be seen from the figure inset, the peak for the state 1 has a maximum at $\sim 490 \mathrm{~nm}$, whereas the peak of state 2 is located at $\sim 486 \mathrm{~nm}$. Thus, in contrast to the red shift in the emission spectra, the excitation spectrum shows a blue-shifted peak of state $\mathbf{2}$ with respect to $\mathbf{1}$ (see Table 2). It should be emphasized

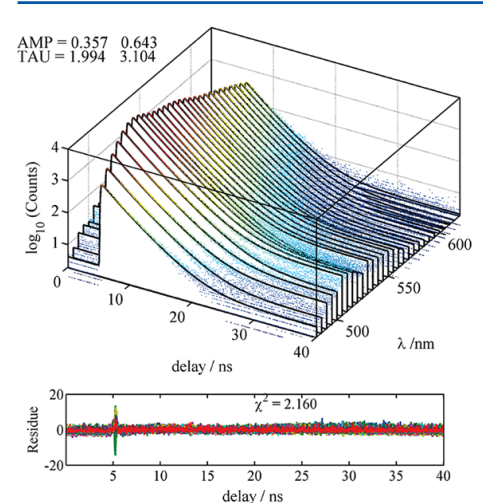

(a)

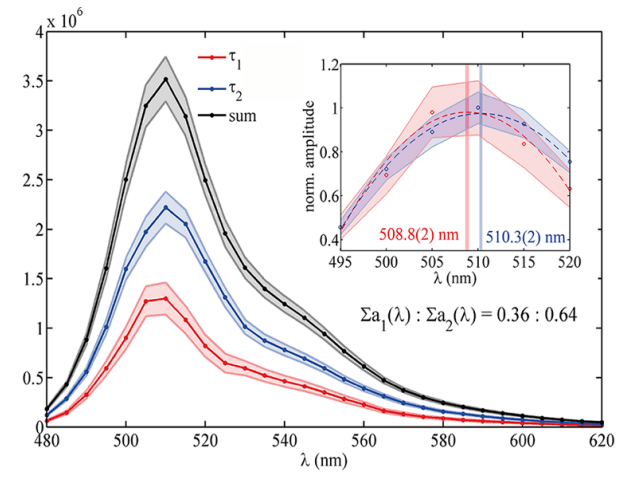

(b)

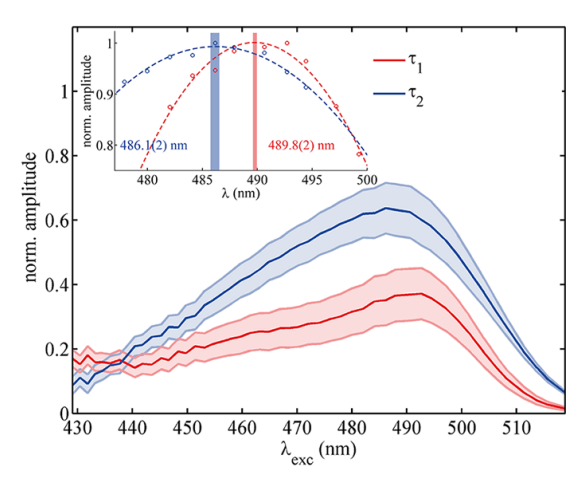

(c)

Figure 3. (a) Amplitudes $a_{1}(\lambda)$ and $a_{2}(\lambda)$ of the two lifetime components obtained by globally fitting a biexponential decay model $\left(I(t, \lambda)=\frac{a_{1}(\lambda)}{\tau_{1}} \exp \left(-\frac{t}{\tau_{1}}\right)+\frac{a_{2}(\lambda)}{\tau_{2}} \exp \left(-\frac{t}{\tau_{2}}\right)\right)$ to the spectrally resolved fluorescence decay data recorded in the EGFP solution at $\mathrm{pH} 9.0$. (b) Emission spectra of $\mathbf{1}$ (red) and $\mathbf{2}$ (blue). The inset shows the spectra normalized to their maximum values, and the solid lines show fitted parabolas around the peaks. The maxima of the two parabolas are indicated with dotted lines. (c) Excitation spectra of 1 (red) and $\mathbf{2}$ (blue), respectively. The embedded graph shows the excitation spectra of the two states normalized to their maximum values, and the dashed lines show the fitted parabolas around the peaks. 
here that the excitation and emission spectra of the two states are highly overlapping and inseparable at room temperature, which makes the lifetime-based correlation indispensable to quantify the transition between the two bright states.

High-resolution X-ray diffraction data ${ }^{29,31}$ of EGFP report the existence of two different conformers of E222 in significant populations. In one of the conformational states, the $\mathrm{OH}$ group of E222 forms a strong and a weak hydrogen bond with S205 and $\mathrm{T} 65$ residues, respectively. A single bond rotation about the $\mathrm{C}_{\gamma}$ of E222 disrupts the H-bond with S205 while strengthening the H-bond with T65, giving rise to a second conformer (as shown in Figure 4). This new conformation prevents ionization of E222 owing to a stronger H-bond with T65, which, in turn, stabilizes the anionic chromophore core $p$-hydroxybenzylide-

(a)

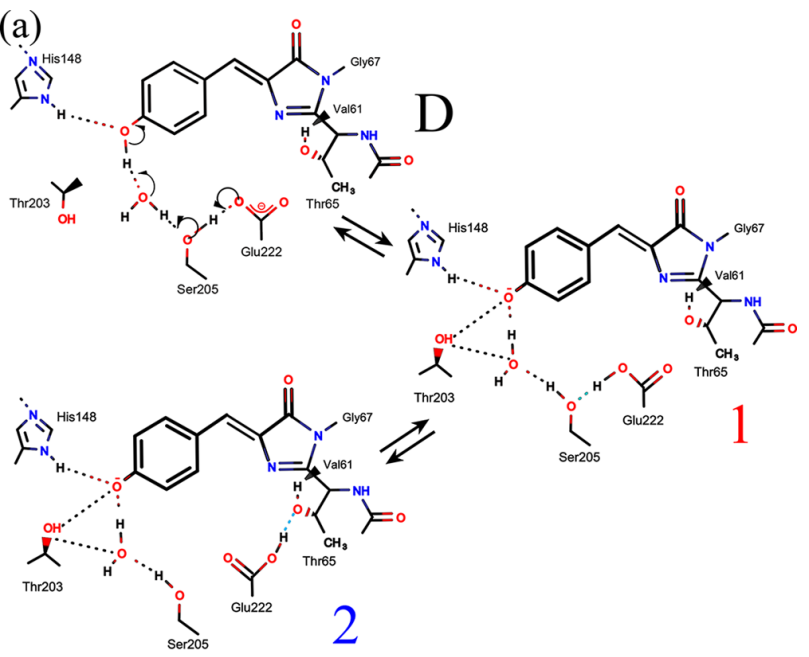

(b)

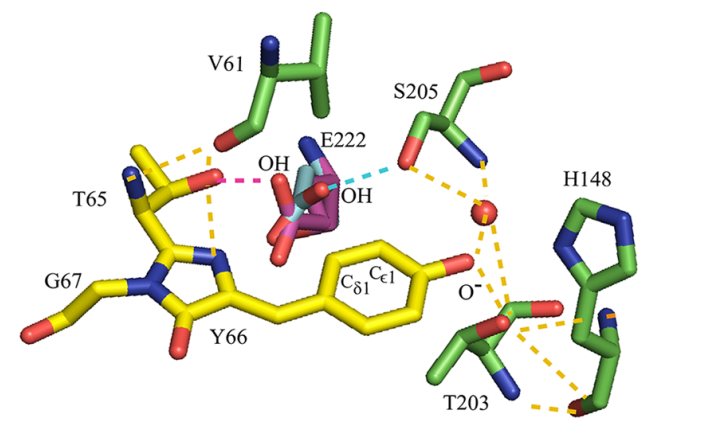

Figure 4. (a) Scheme for the interconversion among the three states D, 1, and 2. The dashed lines show the hydrogen bonds. Water 413, ${ }^{29}$ which is a main part of protonation/deprotonation channel of the chromophore, is also shown here. The E222 resides in anionic form in state $\mathbf{D}$. The hydrogen bond of this residue in the two fluorescent states is highlighted in cyan. The hydrogen bond between the $\mathrm{OH}$ of T65 and carbonyl group of V61 is also shown here. The rotation of this $\mathrm{OH}$ group reduces the steric hindrance between the $\mathrm{C}_{\gamma}\left(\mathrm{CH}_{3}\right.$ group) and E222 in state $\mathbf{2}$ and also promotes a hydrogen bond between E222 and T65. (b) Three-dimensional view of the HBDIchromophore (yellow backbone) and the surrounding amino acids in EGFP as obtained from the crystal structure 4EUL. ${ }^{29}$ The dotted lines represent the hydrogen bonds between the polar groups $(\mathrm{O}, \mathrm{OH}, \mathrm{N}$, and $\mathrm{NH}$ ) of the neighboring residues. The two rotamers of E222 are color coded in cyan ( $30 \%$ occupancy) and magenta ( $70 \%$ occupancy). The hydrogen bonds formed by the $\mathrm{OH}$ group of the two conformations are also color coded. Table 3 presents a comparison of the distances between the E222 OH group with the surrounding atoms, indicating the hydrogen bonds $(\mathrm{H})$. neimidazolone $\left(\mathrm{HBDI}^{-}\right) \cdot{ }^{39}$ Because the $\mathrm{OH}$ group of the T65 is rotated due to steric hindrance by the adjacent methyl group, it prevents close contact between the $\mathrm{C}_{\gamma}$ of T65 and E222 carboxylate, which additionally makes the second conformation more stable $(\sim 70 \% \text { occupancy in crystal data })^{29}$

The state occupancy values can be further explained by considering the distance of the E222 OH group (which has a net negative charge) to the phenyl ring of $\mathrm{HBDI}^{-}$(see Table 3). In its second conformation, this $\mathrm{OH}$ group rotates away

Table 3. Distance between the E222 OH Group and the Surrounding Atoms

\begin{tabular}{cccccc} 
state & $\mathrm{OH}_{\mathrm{S} 205}$ & $\mathrm{OH}_{\mathrm{T} 65}$ & $\mathrm{O}_{\mathrm{Y} 66}^{-}$ & $\mathrm{C}_{\delta 1}$ & $\mathrm{C}_{\epsilon 1}$ \\
$\mathbf{1}$ & $2.7 \AA(\mathrm{H})$ & $3.3 \AA$ & $5.1 \AA$ & $3.4 \AA$ & $3.3 \AA$ \\
$\mathbf{2}$ & $3.7 \AA$ & $2.7 \AA(\mathrm{H})$ & $6.4 \AA$ & $4.5 \AA$ & $4.3 \AA$ \\
\hline
\end{tabular}

from the tyrosine in the $\mathrm{HBDI}^{-}$core, reducing the electrostatic and steric repulsive forces. This leads to the higher state occupancy of this conformer, so that we relate it to state $\mathbf{2}$ in our three-state model. The existence of the two conformers of E222 and the resultant changes in the hydrogen bonding network result in the presence of two fluorescent states of the $\mathrm{HBDI}^{-}$core. Further, the single bond rotation about the $\mathrm{C}_{\gamma}$ of E222 changes the charge distribution around the $\mathrm{HBDI}^{-}$core, which can result in bending of its backbone due to electrostatic repulsion. It can also change the local static electric field seen by the chromophore, which will lead to a change of its photophysical properties (spectra ${ }^{40}$ and lifetime $\tau_{\mathrm{ff}}$, as seen in our results).

The complete scheme is presented in Figure 4 and is closely related to the earlier reported three-state model of wtGFP..$^{33,39,41,42}$ It shows that state 2 can be reached from the protonated dark state $\mathbf{D}$ only through the intermediate state $\mathbf{1}$. This compels us to consider the three-state consecutive reaction model shown in the inset of Figure 2. State $\mathbf{1}$ is similar in structure to the intermediate state $\mathbf{I}$ in wt-GFP that exhibits an emission maximum at $508 \mathrm{~nm} .{ }^{41}$ Further, deprotonation of HBDI essentially takes place through proton diffusion to E222 that requires a hydrogen bond between E222 and S205, which is only feasible when E222 is in its first conformational state, as shown in Figure $4 .{ }^{43}$ The high occupancy of state $\mathbf{2}$ is only possible if the dynamic switching between $\mathbf{2}$ and $\mathbf{1}$ is faster than that between $\mathbf{1}$ and $\mathbf{D}$. In fact, the rates obtained from FLCS show that the former is an order of magnitude faster and within the error limits, and the obtained occupancy values are in good agreement with those of the crystal data. ${ }^{29}$ Moreover, substituting E222 by histidine in EGFP results in monoexponential fluorescence decay with a lifetime value of $2.85 \mathrm{~ns}$, as was observed by Kirk et al. ${ }^{44}$ This shows that the presence of the two conformers of E222 indeed leads to the biexponential fluorescence decay in EGFP.

The transitions between the dark state $\mathbf{D}$ and the fluorescent state in EGFP is known to be associated with the protonation/ deprotonation dynamics of $\mathrm{HBDI}^{-}$. Their rates were reported earlier by Haupts et al. to be on the order of $10^{4}-10^{5} \mathrm{~s}^{-1}$ using conventional FCS. ${ }^{45}$ At high $\mathrm{pH}$ (from $\mathrm{pH} 8$ to 11 ), they relate the dark state transitions in the measurements to internal protonation/deprotonation dynamics inside of the $\beta$-barrel. However, our work finds faster transition rates independent of the excitation powers used $\left(\sim 4\right.$ to $\left.\sim 200 \mathrm{~kW} \mathrm{~cm}^{-2}\right)$. The main reason for this discrepancy, which is more interesting in the context of performing FLCS on such complex luminescent 
systems, is related to the two nonzero eigenvalues of the threestate model considered here. By introducing the relations $k_{\mathrm{f}}=$ $k_{1 \rightarrow 2}+k_{2 \rightarrow 1}$ and $k_{\mathrm{s}}=k_{1 \rightarrow \mathrm{d}}+k_{\mathrm{d} \rightarrow 1}$ and making the simplifying assumptions $k_{1 \rightarrow 2} \approx k_{2 \rightarrow 1}, k_{1 \rightarrow \mathrm{d}} \approx k_{\mathrm{d} \rightarrow 1}$, these eigenvalues can be written as

$$
\lambda_{2,3}=-\frac{1}{2}\left(k_{\mathrm{f}}+k_{\mathrm{s}}\right)\left[1 \mp \sqrt{1-\frac{3 k_{\mathrm{f}} k_{\mathrm{s}}}{\left(k_{\mathrm{f}}+k_{\mathrm{s}}\right)^{2}}}\right]
$$

in accordance with our results. For the case that $k_{\mathrm{f}}$ is significantly larger than $k_{\mathrm{s}}, k_{\mathrm{f}} \gg k_{\mathrm{s}}$, this expression leads to the following approximate eigenvalues

$$
\lambda_{2} \approx-k_{\mathrm{f}} \quad \lambda_{3} \approx-\frac{3}{4} k_{\mathrm{s}}
$$

This demonstrates that if one performs conventional FCS on a three-state system with two bright states with distinct brightness $\left(\kappa_{1} \neq \kappa_{2}\right)$ and interconversion kinetics that takes place on two significantly different time scales $\left(k_{1 \rightarrow 2}+k_{2 \rightarrow 1} \gg\right.$ $\left.k_{1 \rightarrow \mathrm{d}}+k_{\mathrm{d} \rightarrow 1}\right)$, one observes two photophysical time constants: a fast component that represents the switching between the two bright states and a slow component that is $\sim 25 \%$ slower than the relaxation time between one of the bright states and the dark state. The situation becomes even more involved if the protonation rate is faster than the deprotonation rate $\left(k_{1 \rightarrow \mathrm{d}}>\right.$ $\left.k_{\mathrm{d} \rightarrow 1}\right)$, i.e., for $\mathrm{pH}$ values lower than the $\mathrm{p} K_{\mathrm{a}}$ of $\operatorname{EGFP}(\sim 5.7) .{ }^{46}$ In that case, one underestimates the slow relaxation rate $k_{\mathrm{s}}$ as approximated by $\lambda_{3}$ even more than by only $25 \%$. In general, the discussion above also holds true in the reverse situation, i.e., when the transition between the two bright states is significantly slower than the transition between one of the bright states and the dark state. In contrast, FLCS does obtain the correct transition rate constants between all states.

Several groups have focused on measuring the excitation/ emission spectra of the different bright states of $\mathrm{HBDI}^{-}$, for example, by performing high-resolution optical spectroscopy and hole-burning at cryogenic temperatures on mutants of GFP. ${ }^{33,34,39,42,47-49}$ These experiments and parallel X-ray diffraction studies lead to an excellent understanding of the relation between the protein's structure and hydrogen bond network and the optical properties of the chromophore. In particular, the hole-burning experiments at low temperatures on S65T mutant revealed two absorption peaks at 495 and 489 nm. ${ }^{34}$ They were related to two structures similar to states $\mathbf{1}$ and $\mathbf{2}$ in our model. ${ }^{48}$ In comparison to wt-GFP, whose only absorption peak is at $477 \mathrm{~nm},{ }^{33}$ the absorption spectrum of $\mathbf{2}$ is red-shifted. This is due to the orientation of the $\mathrm{T} 65 \mathrm{OH}$ group dipole that is roughly perpendicular to the transition dipole of the chromophore. ${ }^{50}$ In the case of state 1 , the E222 residue rotates away from the $\mathrm{T} 65 \mathrm{OH}$, which in turn reduces the angle between the $\mathrm{OH}$ dipole and the plane of the chromophore, contributing to a slight blue shift. In contrast, this reorientation results in a weakening of the hydrogen bond between the imidazolidine ring of the chromophore and the $\mathrm{OH}$ group that might lead to a red shift in its excitation spectrum. The result of both contrasting effects leads to the observed net red shift of $\mathbf{1}$ with respect to 2 . The spectral results of our experiments are also in good agreement with a theoretical study establishing a relation between the optical properties and the structure of $\mathrm{HBDI}^{-}$in different states of EGFP. ${ }^{50}$ The disappearance of the biexponential fluorescence decay following a point mutation of E222 and the similarity of the determined excitation spectra with those of the hole-burning experiments on S65T mutant strongly support our three-state scheme suggested.

This work presents the first experimental measurements of fast photophysical transitions in a molecule using FLCS. In the case of EGFP, these transitions are driven by rotational isomerization of an amino acid in close contact with the chromophore. Although the resultant change in local electric field and charge distribution around the chromophore do not sensibly affect both emission spectra at room temperature, the markedly different excited-state lifetimes can be used to study the microsecond dynamics. The ability to separate lifetimespecific intensity correlations reveals the unique potential of FLCS as a tool to investigate such rapid transitions. This opens up a broad range of potential applications where fluorescence lifetime changes can be directly linked with, e.g., fluctuations in electron transfer rates in complex biomolecules, ${ }^{13,51}$ local density fluctuations in a polymer matrix, ${ }^{52}$ nanoscale motion of a fluorescent molecule close to metallic structures, ${ }^{38,53,54}$ or nanomechanical resonator motion in the quantum regime, where one deals with oscillation frequencies in the range from $0.1 \mathrm{kHz}$ to $10 \mathrm{MHz} .^{55}$ As we have also shown, if one has not only a simple transition between a bright and a dark state but also rapid transitions between bright substates, conventional FCS experiments will yield a biased estimation of the transition rate constants. In such cases, FLCS is an indispensable tool for estimating these rates accurately. This prompts re-evaluation of the photophysics of complex systems that show multiexponential fluorescence decay. In summary, we hope that FLCS will find manifold applications from physics to life sciences.

\section{ASSOCIATED CONTENT}

\section{Supporting Information}

The Supporting Information is available free of charge on the ACS Publications website at DOI: 10.1021/acs.jpclett.7b02707.

Materials and methods, theory of FLCS, three-state reversible reaction fitting model, FLCS results at various $\mathrm{pH}$ values, and additional experimental results (PDF)

\section{AUTHOR INFORMATION}

\section{Corresponding Author}

*E-mail: nkaredla@physik3.gwdg.de. Phone: +49 (0)551 3913833. Fax: +49 (0)551 7720.

ORCID $\odot$

Ingo Gregor: 0000-0002-1775-2159

Jörg Enderlein: 0000-0001-5091-7157

Narain Karedla: 0000-0002-7891-3825

Notes

The authors declare no competing financial interest.

\section{ACKNOWLEDGMENTS}

EGFP was a kind gift from Stefan Jakobs. Funding from the Deutsche Forschungsgemeinschaft (DFG) is gratefully acknowledged (SFB 860, Project A6; SFB 937, Project A5). J.E. and N.K. thank the DFG Cluster of Excellence and DFG research "Center for Nanoscale Microscopy and Molecular Physiology of the Brain (CNMPB)" for financial support. Fruitful discussions with Daja Ruhlandt, Alexey Chizhik, and Martin Andresen are gratefully acknowledged. 


\section{REFERENCES}

(1) Puller, V.; Lounis, B.; Pistolesi, F. Single molecule detection of nanomechanical motion. Phys. Rev. Lett. 2013, 110, 125501.

(2) Tian, Y.; Navarro, P.; Orrit, M. Single molecule as a local acoustic detector for mechanical oscillators. Phys. Rev. Lett. 2014, 113, 135505.

(3) Mazzamuto, G.; et al. Single-molecule study for a graphene-based nano-position sensor. New J. Phys. 2014, 16, 113007.

(4) Muschik, C. A.; Moulieras, S.; Bachtold, A.; Koppens, F. H.; Lewenstein, M.; Chang, D. E. Harnessing vacuum forces for quantum sensing of graphene motion. Phys. Rev. Lett. 2014, 112, 223601.

(5) Eisert, J.; Plenio, M.; Bose, S.; Hartley, J. Towards quantum entanglement in nanoelectromechanical devices. Phys. Rev. Lett. 2004, 93, 190402.

(6) Vitali, D.; Gigan, S.; Ferreira, A.; Böhm, H.; Tombesi, P.; Guerreiro, A.; Vedral, V.; Zeilinger, A.; Aspelmeyer, M. Optomechanical entanglement between a movable mirror and a cavity field. Phys. Rev. Lett. 2007, 98, 030405.

(7) Gil-Santos, E.; Ramos, D.; Martínez, J.; Fernández-Regúlez, M.; García, R.; San Paulo, Á.; Calleja, M.; Tamayo, J. Nanomechanical mass sensing and stiffness spectrometry based on two-dimensional vibrations of resonant nanowires. Nat. Nanotechnol. 2010, 5, 641-645.

(8) Orrit, M.; Bernard, J. Single pentacene molecules detected by fluorescence excitation in a p-terphenyl crystal. Phys. Rev. Lett. 1990, $65,2716$.

(9) Brunel, C.; Tamarat, P.; Lounis, B.; Woehl, J.; Orrit, M. Stark effect on single molecules of dibenzanthanthrene in a naphthalene crystal and in a n-hexadecane Shpol'skii matrix. J. Phys. Chem. A 1999, 103, 2429-2434.

(10) Caruge, J.-M.; Orrit, M. Probing local currents in semiconductors with single molecules. Phys. Rev. B: Condens. Matter Mater. Phys. 2001, 64, 205202.

(11) Dolde, F.; et al. Electric-field sensing using single diamond spins. Nat. Phys. 2011, 7, 459-463.

(12) Veshchunov, I. S.; Mironov, S. V.; Magrini, W.; Stolyarov, V. S.; Rossolenko, A.; Skidanov, V.; Trebbia, J.-B.; Buzdin, A. I.; Tamarat, P.; Lounis, B. Direct evidence of flexomagnetoelectric effect revealed by single-molecule spectroscopy. Phys. Rev. Lett. 2015, 115, 027601.

(13) Yang, H.; Luo, G.; Karnchanaphanurach, P.; Louie, T.-M.; Rech, I.; Cova, S.; Xun, L.; Xie, X. S. Protein conformational dynamics probed by single-molecule electron transfer. Science 2003, 302, 262266.

(14) Gregor, I.; Enderlein, J. Time-resolved methods in biophysics. 3. Fluorescence lifetime correlation spectroscopy. Photochem. Photobiol. Sci. 2007, 6, 13-18.

(15) Rüttinger, S.; Kapusta, P.; Patting, M.; Wahl, M.; Macdonald, R. On the resolution capabilities and limits of fluorescence lifetime correlation spectroscopy (FLCS) measurements. J. Fluoresc. 2010, 20, 105-114.

(16) Orte, A.; Ruedas-Rama, M. J.; Paredes, J. M.; Crovetto, L.; Alvarez-Pez, J. M. Dynamics of water-in-oil nanoemulsions revealed by fluorescence lifetime correlation spectroscopy. Langmuir 2011, 27, 12792-12799.

(17) Ray, K.; Zhang, J.; Lakowicz, J. R. Fluorescence lifetime correlation spectroscopic study of fluorophore-labeled silver nanoparticles. Anal. Chem. 2008, 80, 7313-7318.

(18) Humpolíčková, J.; Benda, A.; Sỳkora, J.; Macháň, R.; Kral, T.; Gasinska, B.; Enderlein, J.; Hof, M. Equilibrium dynamics of spermineinduced plasmid DNA condensation revealed by fluorescence lifetime correlation spectroscopy. Biophys. J. 2008, 94, L17-L19.

(19) Benda, A.; Fagul'ová, V.; Deyneka, A.; Enderlein, J.; Hof, M. Fluorescence lifetime correlation spectroscopy combined with lifetime tuning: New perspectives in supported phospholipid bilayer research. Langmuir 2006, 22, 9580-9585.

(20) Chen, J.; Irudayaraj, J. Fluorescence lifetime cross correlation spectroscopy resolves EGFR and antagonist interaction in live cells. Anal. Chem. 2010, 82, 6415-6421.

(21) Enderlein, J.; Gregor, I. Using fluorescence lifetime for discriminating detector afterpulsing in fluorescence-correlation spectroscopy. Rev. Sci. Instrum. 2005, 76, 033102.
(22) Gärtner, M.; Mütze, J.; Ohrt, T.; Schwille, P. Fluorescence lifetime correlation spectroscopy for precise concentration detection in vivo by background subtraction. European Conference on Biomedical Optics; 2009; p $7368 \_1 \mathrm{~V}$.

(23) Weber, W.; Helms, V.; McCammon, J. A.; Langhoff, P. W. Shedding light on the dark and weakly fluorescent states of green fluorescent proteins. Proc. Natl. Acad. Sci. U. S. A. 1999, 96, 61776182 .

(24) Nienhaus, K.; Nienhaus, G. U. Chromophore photophysics and dynamics in fluorescent proteins of the GFP family. J. Phys.: Condens. Matter 2016, 28, 443001.

(25) Jung, G.; Wiehler, J.; Zumbusch, A. The photophysics of green fluorescent protein: influence of the key amino acids at positions 65 , 203, and 222. Biophys. J. 2005, 88, 1932-1947.

(26) Cui, G.; Lan, Z.; Thiel, W. Intramolecular hydrogen bonding plays a crucial role in the photophysics and photochemistry of the GFP chromophore. J. Am. Chem. Soc. 2012, 134, 1662-1672.

(27) Stepanenko, O. V.; Stepanenko, O. V.; Kuznetsova, I. M.; Verkhusha, V. V.; Turoverov, K. K. Beta-barrel scaffold of fluorescent proteins: folding, stability and role in chromophore formation. Int. Rev. Cell Mol. Biol. 2013, 302, 221.

(28) Park, J. W.; Rhee, Y. M. Electric Field Keeps Chromophore Planar and Produces High Yield Fluorescence in Green Fluorescent Protein. J. Am. Chem. Soc. 2016, 138, 13619-13629.

(29) Arpino, J. A.; Rizkallah, P. J.; Jones, D. D. Crystal structure of enhanced green fluorescent protein to $1.35 \AA$ resolution reveals alternative conformations for Glu222. PLoS One 2012, 7, e47132.

(30) Snellenburg, J. J.; Laptenok, S. P.; DeSa, R. J.; Naumov, P.; Solntsev, K. M. Excited-State Dynamics of Oxyluciferin in Firefly Luciferase. J. Am. Chem. Soc. 2016, 138, 16252-16258.

(31) Royant, A.; Noirclerc-Savoye, M. Stabilizing role of glutamic acid 222 in the structure of Enhanced Green Fluorescent Protein. J. Struct. Biol. 2011, 174, 385-390.

(32) Lelimousin, M.; Noirclerc-Savoye, M.; Lazareno-Saez, C.; Paetzold, B.; Le Vot, S.; Chazal, R.; Macheboeuf, P.; Field, M. J.; Bourgeois, D.; Royant, A. Intrinsic dynamics in ECFP and Cerulean control fluorescence quantum yield. Biochemistry 2009, 48, 1003810046.

(33) Volker, S.; Creemers, T. M. H.; Lock, A. J.; Subramaniam, V.; Jovin, T. M. Three photoconvertible forms of green fluorescent protein identified by spectral hole-burning. Nat. Struct. Biol. 1999, 6, $557-560$.

(34) Creemers, T.; Lock, A.; Subramaniam, V.; Jovin, T.; Völker, S. Photophysics and optical switching in green fluorescent protein mutants. Proc. Natl. Acad. Sci. U. S. A. 2000, 97, 2974-2978.

(35) Hess, S. T.; Sheets, E. D.; Wagenknecht-Wiesner, A.; Heikal, A. A. Quantitative analysis of the fluorescence properties of intrinsically fluorescent proteins in living cells. Biophys. J. 2003, 85, 2566-2580.

(36) Borst, J. W.; Willemse, M.; Slijkhuis, R.; Van Der Krogt, G.; Laptenok, S. P.; Jalink, K.; Wieringa, B.; Fransen, J. A. ATP changes the fluorescence lifetime of cyan fluorescent protein via an interaction with His148. PLoS One 2010, 5, e13862.

(37) Heikal, A. A.; Hess, S. T.; Baird, G. S.; Tsien, R. Y.; Webb, W. W. Molecular spectroscopy and dynamics of intrinsically fluorescent proteins: coral red (dsRed) and yellow (Citrine). Proc. Natl. Acad. Sci. U. S. A. 2000, 97, 11996-12001.

(38) Karedla, N.; Chizhik, A. I.; Gregor, I.; Chizhik, A. M.; Schulz, O.; Enderlein, J. Single-molecule Metal-Induced Energy Transfer (smMIET): Resolving nanometer distances at the single-molecule level. ChemPhysChem 2014, 15, 705-711.

(39) Brejc, K.; Sixma, T. K.; Kitts, P. A.; Kain, S. R.; Tsien, R. Y.; Ormö, M.; Remington, S. J. Structural basis for dual excitation and photoisomerization of the Aequorea victoria green fluorescent protein. Proc. Natl. Acad. Sci. U. S. A. 1997, 94, 2306-2311.

(40) Amat, P.; Nifosì, R. Spectral fine tuning in fluorescent proteins: The case of the GFP-like chromophore in the anionic protonation state. J. Chem. Theory Comput. 2013, 9, 497-508. 
(41) Chattoraj, M.; King, B. A.; Bublitz, G. U.; Boxer, S. G. Ultra-fast excited state dynamics in green fluorescent protein: multiple states and proton transfer. Proc. Natl. Acad. Sci. U. S. A. 1996, 93, 8362-8367.

(42) Palm, G. J.; Zdanov, A.; Gaitanaris, G. A.; Stauber, R; Pavlakis, G. N.; Wlodawer, A. The structural basis for spectral variations in green fluorescent protein. Nat. Struct. Mol. Biol. 1997, 4, 361-365.

(43) Lill, M. A.; Helms, V. Proton shuttle in green fluorescent protein studied by dynamic simulations. Proc. Natl. Acad. Sci. U. S. A. 2002, 99, 2778-2781.

(44) Kirk, W.; Allen, T.; Atanasova, E.; Wessels, W.; Yao, J.; Prendergast, F. Photophysics of EGFP (E222H) mutant, with comparisons to model chromophores: Excited state pKs, progressions, quenching and exciton interaction. J. Fluoresc. 2017, 27, 895-919.

(45) Haupts, U.; Maiti, S.; Schwille, P.; Webb, W. W. Dynamics of fluorescence fluctuations in green fluorescent protein observed by fluorescence correlation spectroscopy. Proc. Natl. Acad. Sci. U. S. A. 1998, 95, 13573-13578.

(46) Bomati, E. K.; Haley, J. E.; Noel, J. P.; Deheyn, D. D. Spectral and structural comparison between bright and dim green fluorescent proteins in Amphioxus. Sci. Rep. 2015, 4, 5469.

(47) Jung, G.; Wiehler, J.; Zumbusch, A. The photophysics of green fluorescent protein: influence of the key amino acids at positions 65 , 203, and 222. Biophys. J. 2005, 88, 1932-1947.

(48) Kummer, A. D.; Kompa, C.; Lossau, H.; Pöllinger-Dammer, F.; Michel-Beyerle, M. E.; Silva, C. M.; Bylina, E. J.; Coleman, W. J.; Yang, M. M.; Youvan, D. C. Dramatic reduction in fluorescence quantum yield in mutants of green fluorescent protein due to fast internal conversion. Chem. Phys. 1998, 237, 183-193.

(49) Heim, R.; Cubitt, A. B.; Tsien, R. Y. Improved green fluorescence. Nature 1995, 373, 663-664.

(50) Laino, T.; Nifosi, R.; Tozzini, V. Relationship between structure and optical properties in green fluorescent proteins: a quantum mechanical study of the chromophore environment. Chem. Phys. 2004, 298, 17-28.

(51) Min, W.; Luo, G.; Cherayil, B. J.; Kou, S.; Xie, X. S. Observation of a power-law memory kernel for fluctuations within a single protein molecule. Phys. Rev. Lett. 2005, 94, 198302.

(52) Vallée, R.; Tomczak, N.; Kuipers, L.; Vancso, G.; Van Hulst, N. Single molecule lifetime fluctuations reveal segmental dynamics in polymers. Phys. Rev. Lett. 2003, 91, 038301.

(53) Girard, C.; Martin, O. J.; Dereux, A. Molecular lifetime changes induced by nanometer scale optical fields. Phys. Rev. Lett. 1995, 75, 3098 .

(54) Seelig, J.; Leslie, K.; Renn, A.; Kühn, S.; Jacobsen, V.; van de Corput, M.; Wyman, C.; Sandoghdar, V. Nanoparticle-induced fluorescence lifetime modification as nanoscopic ruler: demonstration at the single molecule level. Nano Lett. 2007, 7, 685-689.

(55) Poot, M.; van der Zant, H. S. Mechanical systems in the quantum regime. Phys. Rep. 2012, 511, 273-335. 


\section{Supporting Information: Quantifying \\ Microsecond Transition Times Using}

\section{Fluorescence Lifetime Correlation}

\section{Spectroscopy}

Arindam Ghosh, ${ }^{\dagger}$ Sebastian Isbaner, ${ }^{\dagger}$ Manoel Veiga-Gutiérrez, ${ }^{\ddagger}$ Ingo Gregor ${ }^{\dagger}$ Jörg Enderlein, ${ }^{\dagger}$ and Narain Karedla*,†

$\dagger$ †II. Institute of Physics, Georg August University, 37077 Göttingen, Germany

$\ddagger$ PicoQuant GmbH, 12489 Berlin, Germany

E-mail: nkaredla@physik3.gwdg.de

Phone: +49 (0)551 3913833. Fax: +49 (0)551 7720 


\section{S1. EGFP solution preparation:}

Stock solution of $1 \mu \mathrm{M}$ EGFP was dissolved in $100 \mathrm{mM}$ Tris- $\mathrm{HCl}$ with $150 \mathrm{mM} \mathrm{NaCl}$ at pH 7.0. For final sample preparations, we used $100 \mathrm{mM}$ Tris- $\mathrm{HCl}$ with $150 \mathrm{mM} \mathrm{NaCl}$ for $\mathrm{pH}$ values 9.0 and 7.5, whereas 1x PBS with $150 \mathrm{mM} \mathrm{NaCl}$ for $\mathrm{pH}$ 6.0. EGFP protein from the stock solution was diluted in these buffers to nanomolar concentrations for FLCS measurements.

\section{S2. Fluorescence Lifetime Correlation Spectroscopy}

FLCS is a cross-correlation spectroscopy technique that uses fluorescence lifetimes for calculating auto- and cross-correlations similar to conventional dual-color Fluorescence CrossCorrelation Spectroscopy (FCCS), that uses emission colors for discriminating between different fluorophores or fluorescent states. ${ }^{1}$ The core advantage of FLCS is that one can distinguish fluorescence correlations of two or more emitting species that emit in the same spectral range, solely on the basis of their fluorescence lifetimes. Although the complete theory of FLCS has been described in Gregor et al., ${ }^{?}$ we outline the essentials here briefly.

Let us consider a sample emitting fluorescence with $n$ different decay patterns such that the number of photons in each TCSPC channel $(j=1,2,3, \ldots, L)$ is

$$
I_{j}(t)=\sum_{i=1}^{n} c^{(i)}(t) p_{j}^{(i)}
$$

where $p_{j}^{(i)}$ is the discretized probability distribution function of fluorescence photon arrival times with respect to the excitation pulse over the TCSPC channels $j, c^{(i)}$ is the total number of photons corresponding to the decay pattern $i(i=1,2,3, \ldots, n)$ at a given time $t$. We are interested in calculating fluorescence-decay specific two-photon auto- and cross-correlation functions defined by

$$
g_{\alpha \beta}(t)=\left\langle c^{(\alpha)}\left(t_{0}\right) c^{(\beta)}\left(t+t_{0}\right)\right\rangle_{t_{0}}
$$


where $\alpha$ and $\beta$ can take values from 1 to $n$, and the angular brackets represent averaging over $t_{0}$. For that purpose, one has to extract the values $c^{(i)}(t)$ from the measured photon stream. This is performed by calculating statistical filter functions $f_{j}^{(i)}$ such that they satisfy the relations

$$
\left\langle\sum_{j} f_{j}^{(i)} \cdot I_{j}\right\rangle=c^{(i)}
$$

and minimize the relative errors given by

$$
\left\langle\left(\sum_{j} f_{j}^{(i)} \cdot I_{j}-c^{(i)}\right)^{2}\right\rangle
$$

where angular brackets again denote time averaging. The summations are performed over all the TCSPC channels and the averaging over an infinite number of measurements. Using the fact that the photon detection in each TCSPC channel follows Poissonian statistics, these filter functions can be calculated numerically using a weighted quasi-inverse matrix operation, ${ }^{2}$ yielding the following explicit expression for the filter functions

$$
f_{j}^{(i)}=\left[\hat{\boldsymbol{M}} \cdot \operatorname{diag}\left\langle I_{j}\right\rangle^{-1} \hat{\boldsymbol{M}}^{T}\right]^{-1} \cdot \hat{\boldsymbol{M}} \cdot \operatorname{diag}\left\langle I_{j}\right\rangle^{-1}
$$

Here, $\hat{\boldsymbol{M}}_{i j}=p_{j}^{(i)}$ and $\operatorname{diag}\left\langle I_{j}\right\rangle^{-1}$ is an $L \times L$-dimensional matrix with diagonal elements $\left\langle I_{j}\right\rangle^{-1}$. An important point that should be emphasized here is that these filter functions form a dual orthogonal basis to the decay patterns. This means that element-wise multiplication and summation of these filter functions with the fluorescence decay patterns yields an identity matrix. Using these filters, the second order auto- and cross-correlation function from equation 2 can now be expressed as

$$
g_{\alpha \beta}(t)=\sum_{j=1}^{L} \sum_{k=1}^{L} f_{j}^{\alpha} f_{k}^{\beta}\left\langle I_{j}\left(t+t_{0}\right) I_{k}\left(t_{0}\right)\right\rangle_{t_{0}}
$$


For $\alpha=\beta$, we get lifetime auto-correlations which represent the intensity fluctuations of each individual lifetime species, and if $\alpha \neq \beta$, we obtain the cross-correlations of $\alpha$ versus $\beta$, which indicate the probability of detecting a second photon from species $\beta$ at time $t$ after detecting a photon from species $\alpha$. Generally, $g_{\alpha \beta} \neq g_{\beta \alpha}$; the equality occurs only under strict conditions such as pure co-diffusion. For example, for a sample comprising of two molecular species with two distinct fluorescence decay patterns, one thus obtains two lifetime auto-correlation curves, one for each pattern, and two cross-correlation curves for correlations between the patterns.

It is important to emphasize that throughout the above mathematical treatment, we did not include any restriction on the nature of $p_{j}^{(i)}$. Therefore, one can apply FLCS to fluorescence samples exhibiting any form of fluorescence decay kinetics (single- or multi-exponential decay). A straightforward extension of the concept above is to include an additional component with a uniform distribution of probability among the TCSPC channels in order to eliminate background contributions (e.g. dark counts, detector after pulsing) from the final fluorescence auto- and cross-correlations. The theory is generic and has been applied in a variety of works other than FLCS such as fluorescence spectral correlation spectroscopy, ${ }^{3}$ and filtered FCS. ${ }^{4}$

Enhanced Green Fluorescent Protein (EGFP) exhibits a bi-exponential decay behavior. ${ }^{5}$ We measured the fluorescence decay of nanomolar concentrations of EGFP at $\mathrm{pH}$ values of 6.0, 7.5 and 9.0. Figure 1 (a) shows a TCSPC curve obtained from EGFP measurement at pH 9.0. The data was tail-fitted by minimizing the least-squares error using a bi-exponential decay model

$$
I_{j}=\frac{a_{1}}{\tau_{1}} e^{-j \delta / \tau_{1}}+\frac{a_{2}}{\tau_{2}} e^{-j \delta / \tau_{2}}+b
$$

where $j=1, \ldots, L$ refer to the $j^{\text {th }}$ discrete TCSPC time channel, $\delta$ is the bin width of each time channel, $a_{i}$ are the amplitudes, $\tau_{i}$ are the decay constants corresponding to the lifetime 

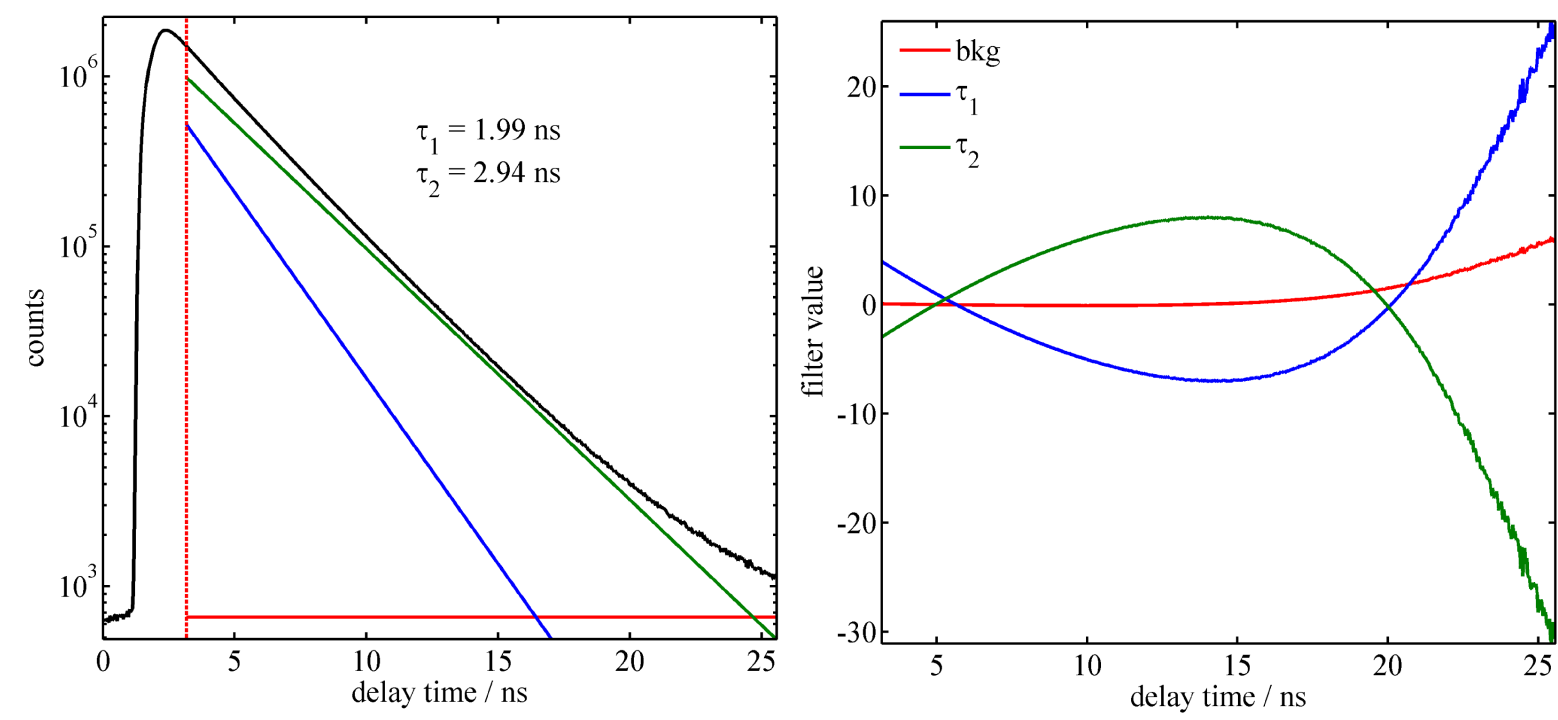

Figure S1: The left figure shows the TCSPC data together with the two lifetime components and a constant offset (solid red line) representing the background signal. The right figure shows the calculated filters using the three components and the TCSPC curve itself as according to equation 5

of each decay process, and $b$ is an offset which arises chiefly due to the dark counts and afterpulsing from the detectors. ${ }^{6}$ The two decay constants 2.0(1) ns and 3.1(2) ns occurred to be independent of $\mathrm{pH}$ (between $\mathrm{pH} 6.0$ and $\mathrm{pH}$ 9.0) and laser intensities (from (4 200) $\left.\mathrm{kW} / \mathrm{cm}^{2}\right)$.

Next, we calculated lifetime-specific filter functions for the two decay components and an additional constant-amplitude component. The latter is used to filter out contributions from background photons which generate a constant offset in the TCSPC curve. Figure 1(a) shows the decay patterns used for calculating the filter functions in (b). The physical interpretation of these functions is as follows: The values of the filter corresponding to the faster decay component are positive (and > 1) in the first few time bins, since the photons arriving at short delay times predominantly contribute to the auto-correlation of the short lifetime species. The filter function corresponding to the longer lifetime component (green curve) has positive values for later time bins, where the long-lifetime component predominantly contributes to auto-correlation. The filter representing the background (red curve) has values close to zero in the time channels up to $10 \mathrm{~ns}$, which means that background events falling into these time 


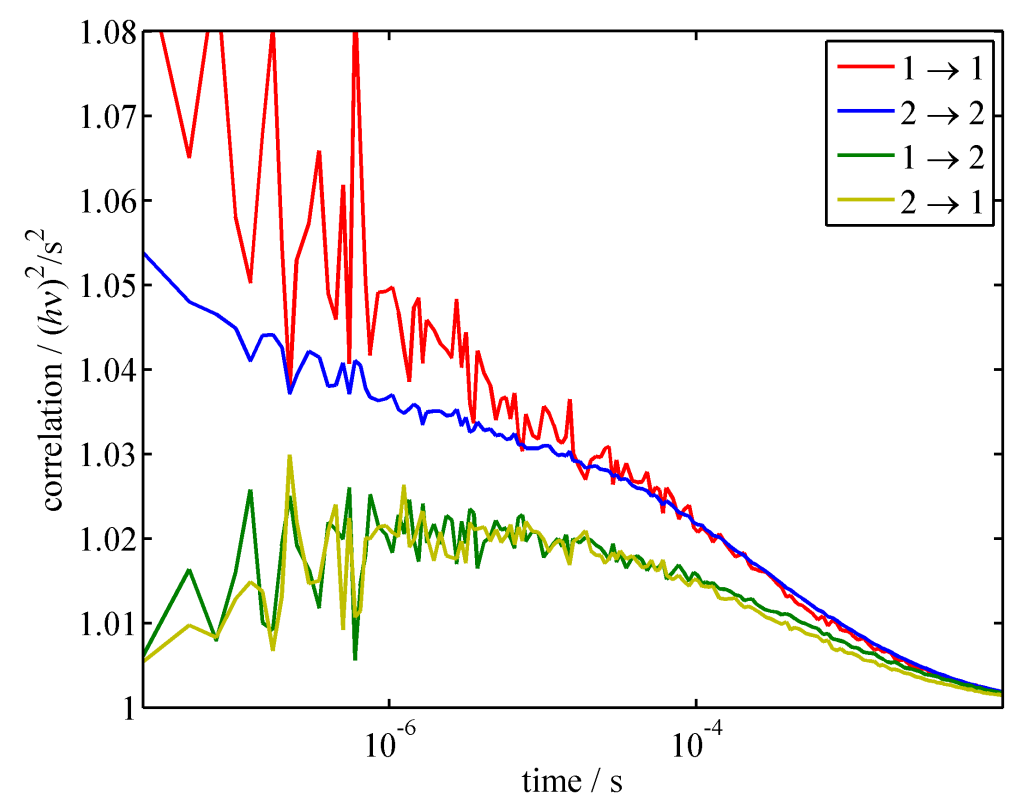

Figure S2: Calculated auto- and cross-correlations for EGFP measurements at pH 9.0 with $\sim 50 \mathrm{~kW} / \mathrm{cm}^{2}$ excitation power. The correlations are normalized using with their amplitudes at $t=0.1 \mathrm{~s}$. The anti-correlation that is visible in the sub-microsecond to microsecond range arises due to the dynamic transitions between the two states.

channels contribute negligibly to the auto- and cross-correlations of and between the two lifetime states. The filter functions are normalized in such a way that their sum in each time channel is one.

Correlation functions are calculated from the asynchronous stream of photon arrival times on a logarithmic correlation time scale using a dedicated algorithm ${ }^{7}$ In FLCS, each photon is additionally weighted, depending on its TCPCS channel where it is recorded, with one of the three filter functions before correlation. Thus, one obtains in general nine different correlation functions (three auto-correlation functions between photons of the same lifetime pattern, and six cross-correlation functions between photons weighted with different lifetime patterns). Of them, of physical interest are only the two auto-correlation (one for each lifetime state) and two cross-correlation (transitions between the two lifetime states) functions related to the fluorescence lifetime patterns, but not those involving the background pattern, see Figure 1. The smallest time bin is equal to the inter-pulse time distance of the 
laser (25 ns).

Figure 2 shows the auto- and cross-correlations of the two fluorescent states of EGFP at pH 9.0. We took an additional TCSPC distribution, as shown by the red horizontal line in the right panel of figure 1, as a third state to eliminate any contribution from background. The anticorrelation seen in both cross-correlation curves on the timescale of $10^{-7} \mathrm{~s}-10^{-6} \mathrm{~s}$ corresponds to a microsecond reversible switching between the two bright states. If the molecule switches randomly in time between the two lifetime states (without a fixed rate), or two populations exist independently, the behavior of the cross-correlation curves will be completely different. In the first case, one would not see any anti-correlation behavior and the cross-correlations would show identical temporal behavior as the auto-correlation curves, and in the later situation one will not observe any cross-correlations due to the randomness of the presence of molecules from two populations at a given time in the focus. The long-time behavior of the correlation curves is governed by photophysics of the chromophore itself, due to the dynamic transitions to a dark protonated state on the timescale of $\sim 10^{-5} \mathrm{~s}$, followed by the diffusion of the EGFP molecules in and out of the excitation focus $\left(\sim 10^{-3} \mathrm{~s}\right)$.

\section{S3. Three-state consecutive reversible-reaction model}

The most exciting property of FLCS is that it can resolve rapid temporal fluctuations of fluorescence lifetimes with single molecule sensitivity, down to the nanosecond timescale. In conjunction with an appropriate model, the correlation information provided by FLCS can be used to determine transition rates and occupation times for the different photophysical states that are connected to the different fluorescence decay patterns. In the current paper, for describing the rapid lifetime and intensity fluctuations observed in EGFP, we use the following three-state model for describing the photophysical behavior of the protein (its validity will be discussed below). It comprises of two bright states that show distinct fluorescence decay rates, and one dark state. These three state are interconnected to each other through two 
consecutive reversible reactions such that $\mathbf{D} \rightleftharpoons \mathbf{1} \rightleftharpoons \mathbf{2}$ as shown in the model in figure 3 . The necessary model parameters to model such a three-state system are the rate constants $k_{1 \rightarrow 2}$ and $k_{2 \rightarrow 1}$ for the transition from $\mathbf{1}$ to $\mathbf{2}$ and $\mathbf{2}$ to $\mathbf{1}$, respectively; and the transition rates $k_{1 \rightarrow \mathrm{d}}$ and $k_{\mathrm{d} \rightarrow 1}$ related to the transitions of the chromophore $p$-hydroxybenzylideneimidazolone (HBDI) between state $\mathbf{1}$ and the dark state $\mathbf{D}$.

If we now denote the probability to find the chromophore in state $\mathbf{D}, \mathbf{1}$, and $\mathbf{2}$ by $n_{\mathrm{d}}, n_{1}$, and $n_{2}$, respectively, such that $n_{1}+n_{2}+n_{\mathrm{d}}=1$, the three rate equations for the temporal evolution of these states can be written in matrix form as

$$
\frac{\mathrm{d} \vec{n}}{\mathrm{~d} t}=\hat{\boldsymbol{T}} \cdot \vec{n}
$$

where $\hat{\boldsymbol{T}}$ is the rate matrix,

$$
\hat{\boldsymbol{T}}=\left(\begin{array}{ccc}
-k_{\mathrm{d} \rightarrow 1} & k_{1 \rightarrow \mathrm{d}} & 0 \\
k_{\mathrm{d} \rightarrow 1} & -k_{1 \rightarrow 2}-k_{1 \rightarrow \mathrm{d}} & k_{2 \rightarrow 1} \\
0 & k_{1 \rightarrow 2} & -k_{2 \rightarrow 1}
\end{array}\right)
$$

and $\vec{n}=\left(n_{\mathrm{d}}, n_{1}, n_{2}\right)^{\mathrm{T}}$ is the state vector. The solution to this system of linear differential equations can be written as

$$
\vec{n}(t)=\sum_{j=1}^{3} \hat{e}_{j}\left(\vec{n}_{0} \cdot \hat{e}_{j}\right) \exp \left(\lambda_{j} t\right)
$$

where $\vec{n}_{0}$ is the initial value of $\vec{n}$ at $t=0$, and the $\lambda_{j}$ and $\hat{e}_{j}$ are the eigenvalues and eigenvectors of the transfer matrix $\hat{\boldsymbol{T}}$, respectively. The eigenvalues are explicitly given by

$$
\lambda_{1}=0, \quad \lambda_{2,3}=\frac{\sigma \pm \delta}{2}
$$




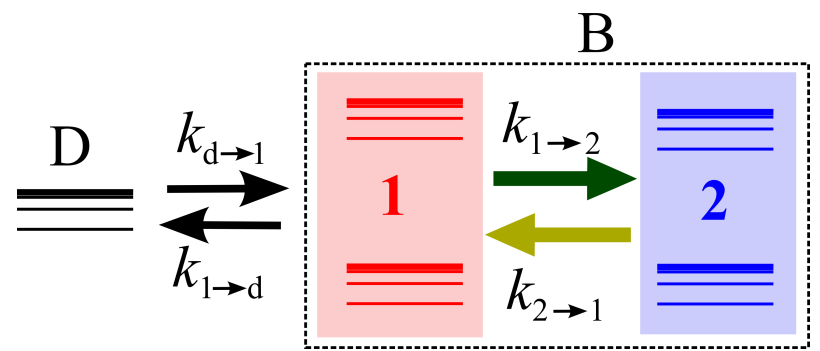

Figure S3: Schematic showing the three-state reversible reaction model for EGFP considered for the data analysis. The protein switches between two fluorescent states $\mathbf{1}$ and $\mathbf{2}$ (collectively shown as $\mathbf{B}$ ). Transition to a third dark state $\mathbf{D}$ take place from $\mathbf{1}$, due to protonation/deprotonation dynamics of $p$-hydroxybenzylideneimidazolone (HBDI)

where

$$
\sigma=-\left(k_{1 \rightarrow 2}+k_{2 \rightarrow 1}+k_{1 \rightarrow \mathrm{d}}+k_{\mathrm{d} \rightarrow 1}\right)
$$

and

$$
\delta^{2}=\sigma^{2}-4\left(k_{1 \rightarrow 2} k_{\mathrm{d} \rightarrow 1}+k_{2 \rightarrow 1} k_{1 \rightarrow \mathrm{d}}+k_{2 \rightarrow 1} k_{\mathrm{d} \rightarrow 1}\right)
$$

The zero value of the first eigenvalue reflects the fact that the sum of all state occupancies is unity. The remaining two eigenvalues are functions of the transition rates between all the three states. Knowing the temporal evolution of the state vector, the correlation functions connected with the the state relaxation are given by

$$
g_{\alpha \beta}^{\text {fast }}(t)=\kappa_{\alpha} \kappa_{\beta} \sum_{j=1}^{3} \hat{e}_{j, \alpha} \hat{e}_{j, \beta} \exp \left(\lambda_{j} t\right)
$$

where $\kappa_{\alpha}, \kappa_{\beta}$ are coefficients taking into account the relative brightness of the fluorescent states $\alpha$ and $\beta$, respectively. Provided that the relaxation times for $\vec{n}(t)$ (inverse values of the non-zero eigenvalues of the transfer matrix) are much faster than the average diffusion 
time of a molecule through the detection volume, the full correlation curves are given by

$$
g_{\alpha \beta}(t)=g_{\alpha \beta}^{\infty}(t)+\frac{w_{1}^{2} w_{2}}{\left(w_{1}^{2}+4 D t\right)\left(w_{2}^{2}+4 D t\right)^{1 / 2}} \cdot g_{\alpha \beta}^{\text {fast }}(t)
$$

where we have assumed a three-dimensional Gaussian shape of the detection volume, with $w_{1}$ and $w_{2}$ being the semi-axes in the lateral and axial direction, respectively, and where $g_{\alpha \beta}^{\infty}$ is a constant offset, and $D$ the diffusion coefficient of the molecule.

The infinite-time limit of $\vec{n}(t)$ describes the steady state occupancies at equilibrium. For the three-state model considered above, these steady state occupancies are given by

$$
\vec{n}(t=\infty)=\frac{1}{\eta}\left(\begin{array}{l}
k_{1 \rightarrow \mathrm{d}} k_{2 \rightarrow 1} \\
k_{\mathrm{d} \rightarrow 1} k_{2 \rightarrow 1} \\
k_{\mathrm{d} \rightarrow 1} k_{1 \rightarrow 2}
\end{array}\right)
$$

where $\eta=k_{1 \rightarrow \mathrm{d}} k_{2 \rightarrow 1}+k_{\mathrm{d} \rightarrow 1} k_{2 \rightarrow 1}+k_{\mathrm{d} \rightarrow 1} k_{1 \rightarrow 2}$. This leads to the direct result that the state occupancies of the two fluorescent states at equilibrium is $n_{1}: n_{2}=k_{2 \rightarrow 1}: k_{1 \rightarrow 2}$. The theory developed above can be extended on similar lines to describe any multi-state transition scheme as per requirements.

The experimentally determined auto-correlation of $\mathbf{1}$ shows a strong variation around its mean (fitted solid line). This is due to the low number of photons recorded from this state (total signal contribution of only $25 \%$ after the cutoff shown in figure 1 (a)). Also, the deviations form the mean for the different correlation curves show a strong correlation. This can be easily understood when realizing that the sum of all FLCS correlation curves reproduces the auto-correlation of a conventional FCS measurement (shown by the inset in Figure 1). Thus, any deviation from the mean of one FLCS curve in one direction has to be compensated by opposite deviations in the other FLCS curves.

The curves were fitted with two-photon correlation functions $g_{\alpha \beta}$ derived from the threestate model. The kinetics of the rapid correlation change at short times scales (shorter than 
the diffusion time of the molecule, $t \leq 10^{-4} \mathrm{~s}$ ) is determined by the rate constants $k_{1 \rightarrow 2}$ and $k_{2 \rightarrow 1}$ of the conversions between the two fluorescent states, and the rate constants $k_{1 \rightarrow \mathrm{d}}$ and $k_{\mathrm{d} \rightarrow 1}$ for the transitions between the fluorescent state $\mathbf{1}$ and the dark state $\mathbf{D}$. The rationale for a three-state consecutive reversible reaction model will be addressed in the main text. Fitting of the rate constants was done in the following way: FLCS calculations were done on bunches of one million photons and then added up to the final correlation curves. For determining the fit errors, we used bootstrapping by randomly omitting bunches form the full photon stream, and the resulting four correlation curves were globally fitted with our kinetic model.
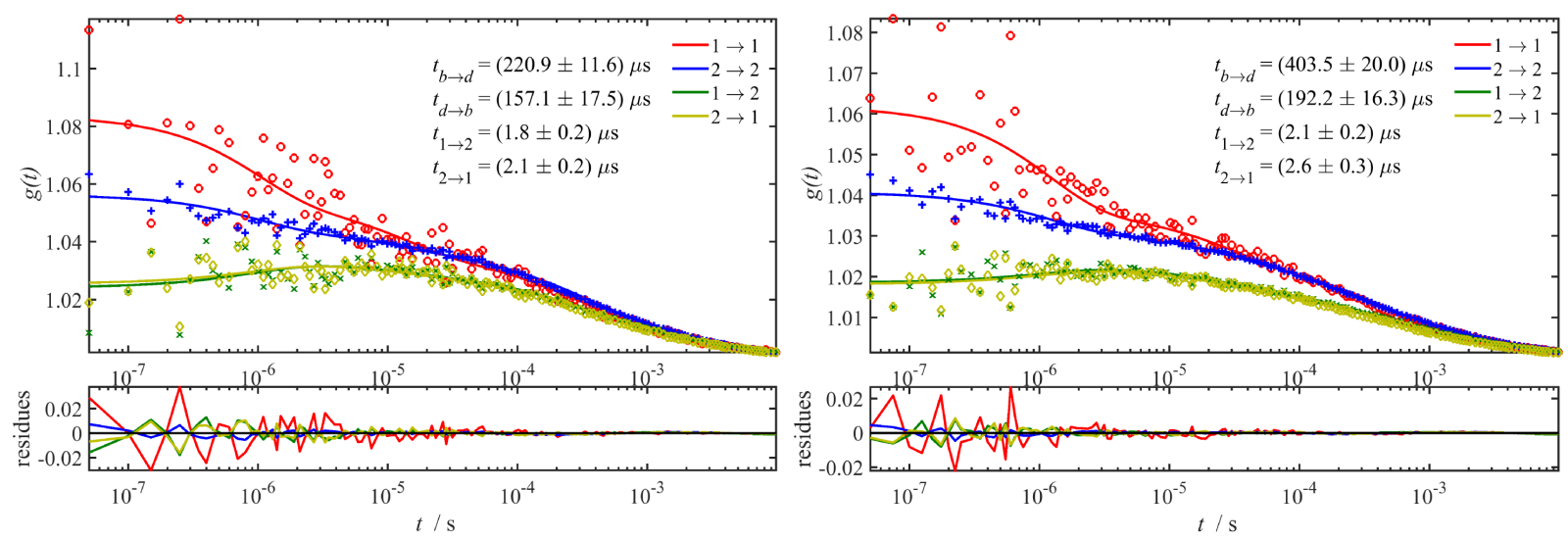

Figure S4: Globally fitted auto- and cross-correlation curves for EGFP at pH 7.5 (left) and 9.0 (right) using the three-state model. The half-time for each transition is listed in the graph itself. The measurements were performed using excitation powers of $\sim 25 \mathrm{kWcm}^{-2}$ and $\sim 50 \mathrm{kWcm}^{-2}$, respectively.

\section{S4. Spectrally resolved fluorescence decay measurements}

Spectrally resolved fluorescence decay measurements have been collected in a FT300 spectrometer (PicoQuant GmbH, Berlin, Germany) equipped with a TH260-P single photon timing board and a hybrid HPD-40 detector. Excitation was performed with a LDH-PC-470 diode laser at $25 \mathrm{MHz}$, driven by a PDL-820 controller. Each decay was collected at magic angle for $17 \mathrm{~s}$ and band pass of $5 \mathrm{~nm}$, in the range from $480 \mathrm{~nm}$ to $620 \mathrm{~nm}$ with a $5 \mathrm{~nm}$ step. 
The count rate at all wavelengths acquired was kept below $2 \%$ of the excitation rate to avoid any dead-time based artifacts. ${ }^{8}$

\section{S5. Additional Experimental Results}
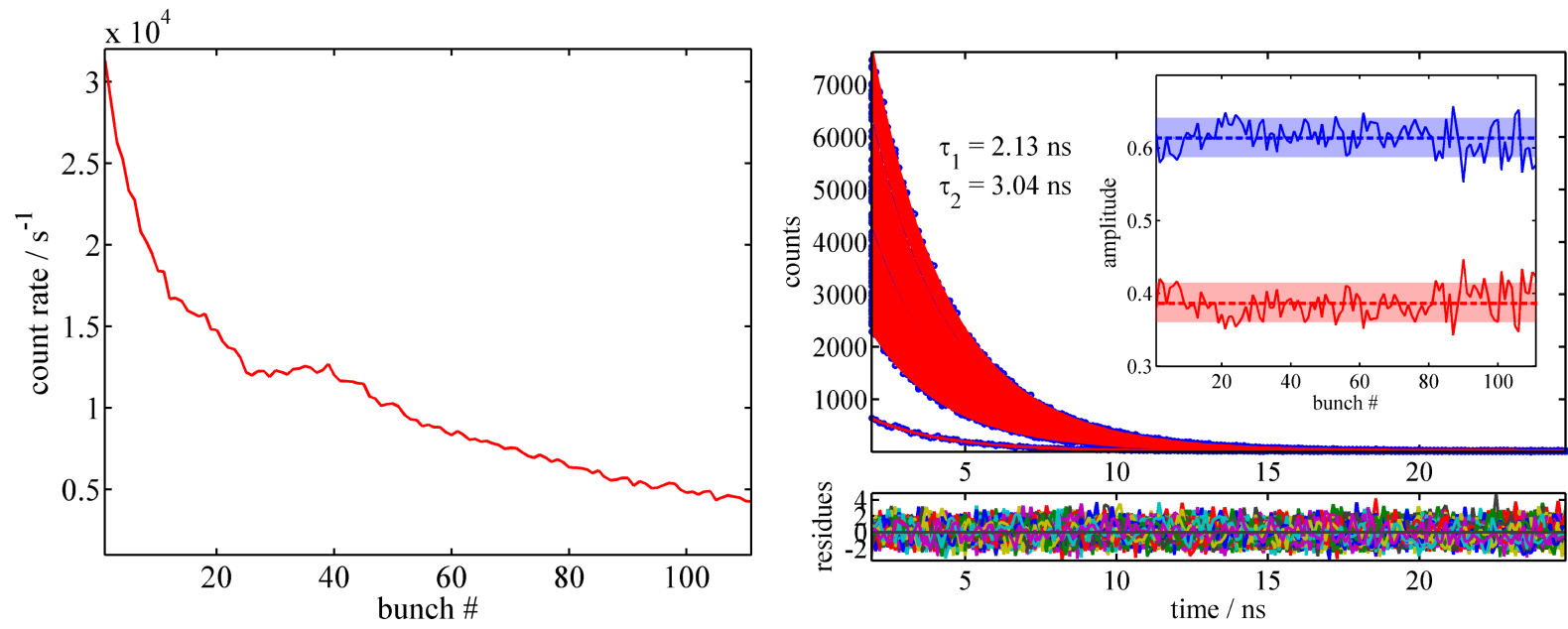

Figure S5: This figure shows that there is no effect of photobleaching on the two lifetime components. The left figure shows the rate of photons for each bunch that corresponds to $\approx 10 \mathrm{~s}$. The right figure shows the arrival time histograms of the photons tail fitted with a bi-exponential model given by equation (solid red curves). The curves are globally fitted and the two lifetime values are indicated $\left(\tau_{1}=2.1 \mathrm{~ns}\right.$ and $\left.\tau_{2}=3 \mathrm{~ns}\right)$. The inset shows the ratio of the photons from both components corresponding for each bunch comprising one million photons (red for $\tau_{1}$ and blue for $\tau_{2}$ ). The solid lines indicate the average and the shaded region marks the standard deviation of the amplitudes. The mean values are $\left\langle a_{1}\right\rangle=0.39$ and $\left\langle a_{2}\right\rangle=0.61$. 

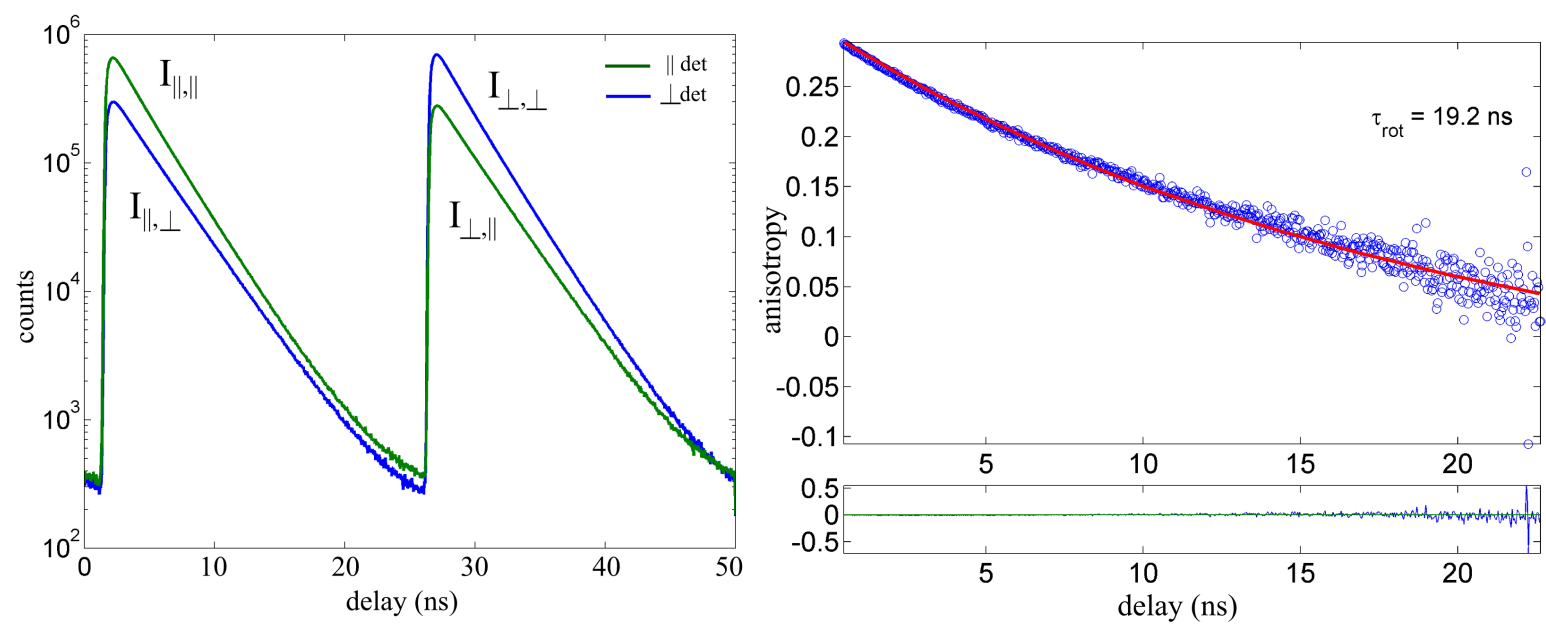

Figure S6: Time-resolved anisotropy measurements. The left figure shows the TCSPC data recorded with pulsed interleaved excitation with a horizontally and vertically polarized laser sources, each with a repetition rate of $20 \mathrm{MHz}$. The fluorescence is split by a polarizing beamsplitter and focused onto two detectors. Each detector measures two consecutive fluorescence decay curves within one complete excitation cycle, one corresponding to the laser which is parallel in polarization to itself (higher amplitude) and the other which is orthogonal to it (lower amplitude). The four TCSPC curves thus recorded can be named as $I_{\|,\|}, I_{\|, \perp}, I_{\perp, \|}, I_{\perp, \perp}$ where the first symbol represents the polarization of the laser with respect to a fixed $\|$ and $\perp$ orientation in the laboratory reference frame and the second symbol marks the orientation for the detection. The time resolved anisotropy $\mathrm{r}(\tau)$ is calculated as:

$$
r(\tau)=\frac{\sqrt{I_{\|,\|}(\tau) \times I_{\perp, \perp}(\tau)}-\sqrt{I_{\|, \perp}(\tau) \times I_{\perp, \|}(\tau)}}{\sqrt{I_{\|,\|}(\tau) \times I_{\perp, \perp}(\tau)}+2 \sqrt{I_{\|, \perp}(\tau) \times I_{\perp, \|}(\tau)}}
$$

The left figure shows the time-resolved anisotropy decay of fluorescence collected from EGFP in solution at $21^{\circ} \mathrm{C}$ (blue circles). The solid red line shows the exponential decay fit which gives us the rotational diffusion time $\tau_{\text {rot }}=19.2 \mathrm{~ns}$. Approximating the EGFP molecule as a sphere and using Stokes-Einstein relation, the hydrodynamic radius can be calculated as:

$$
R_{h}=\sqrt[3]{\frac{3 k_{B} T \tau_{r o t}}{4 \pi \eta}}
$$

where $k_{B}$ is the Boltzmann constant, and $\eta$ is the viscosity of the solution at temperature $T$. Assuming the buffer's viscosity to be equal to water, we obtain a hydrodynamic radius $R_{h} \approx 2.6 \mathrm{~nm}$, which is close to the values known in literature. ${ }^{9}$ This shows that EGFP exists as a monomer in our experiments. 


\section{References}

(1) Böhmer, M.; Wahl, M.; Rahn, H.-J.; Erdmann, R.; Enderlein, J. Time-resolved fluorescence correlation spectroscopy. Chem. Phys. Lett. 2002, 353, 439-445.

(2) Enderlein, J.; Erdmann, R. Fast fitting of multi-exponential decay curves. Opt. Commun. 1997, 134, 371-378.

(3) Benda, A.; Kapusta, P.; Hof, M.; Gaus, K. Fluorescence spectral correlation spectroscopy (FSCS) for probes with highly overlapping emission spectra. Opt. Exp. 2014, 22, 29732988.

(4) Felekyan, S.; Kalinin, S.; Sanabria, H.; Valeri, A.; Seidel, C. A. Filtered FCS: Species Auto-and Cross-Correlation Functions Highlight Binding and Dynamics in Biomolecules. ChemPhysChem 2012, 13, 1036-1053.

(5) Hess, S. T.; Sheets, E. D.; Wagenknecht-Wiesner, A.; Heikal, A. A. Quantitative analysis of the fluorescence properties of intrinsically fluorescent proteins in living cells. Biophys. J. 2003, 85, 2566-2580.

(6) Enderlein, J.; Gregor, I. Using fluorescence lifetime for discriminating detector afterpulsing in fluorescence-correlation spectroscopy. Rev. Sci. Instrum. 2005, 76, 033102.

(7) Wahl, M.; Gregor, I.; Patting, M.; Enderlein, J. Fast calculation of fluorescence correlation data with asynchronous time-correlated single-photon counting. Opt. Exp. 2003, $11,3583$.

(8) Isbaner, S.; Karedla, N.; Ruhlandt, D.; Stein, S. C.; Chizhik, A.; Gregor, I.; Enderlein, J. Dead-time correction of fluorescence lifetime measurements and fluorescence lifetime imaging. Opt. Exp. 2016, 24, 9429-9445.

(9) Konzack, S.; Thies, E.; Marx, A.; Mandelkow, E.-M.; Mandelkow, E. Swimming against 
the tide: mobility of the microtubule-associated protein tau in neurons. J. Neurosci. 2007, 27, 9916-9927. 


\subsection{Manuscript: Fluorescence lifetime correlation spec- troscopy : Basics and applications}

In this review article [102], we recapitulate the fundamentals and instrumentation as well as biological and spectroscopic applications of FLCS. The applications of FLCS include quantification of protein-protein interactions [165], reduction of spectral crosstalk in live-cell studies [166], investigation of lifetime changes of a fluorophore in close proximity to silver nanoparticles [167], FLCS experiments in conjunction with lifetime tuning [168] for studying diffusion in SLBs, investigation of DNA compaction by spermine [169], proton transfer reactions [105], and, along with FRET, quantification of kinetic rates of interconversion in Syntaxin 1 [170]. Furthermore, we furnish the details of an interesting extension of FLCS, known as two-dimensional FLCS [171], and discuss also the utilization of FLCS in STED microscopy [106].

The mini-review entitled "Arindam Ghosh, Narain Karedla, Jan Christoph Thiele, Ingo Gregor, and Jörg Enderlein. Fluorescence lifetime correlation spectroscopy: Basics and applications. Methods, 140:32-39, 2018" is presented below. A.G. co-wrote the minireview with inputs from other authors.

Reproduced with permission, copyright 2018 The Authors, published by Elsevier Inc.

The published mini-review is currently available at https://www.sciencedirect.com/ science/article/pii/S104620231730230X 


\title{
Fluorescence lifetime correlation spectroscopy: Basics and applications
}

\author{
Arindam Ghosh, Narain Karedla, Jan Christoph Thiele, Ingo Gregor, Jörg Enderlein* \\ Third institute of Physics - Biophysics, Georg August University, 37077 Göttingen, Germany
}

\section{A R T I C L E I N F O}

\section{Article history:}

Received 21 November 2017

Received in revised form 9 February 2018

Accepted 10 February 2018

Available online 16 February 2018

\section{Keywords:}

Fluorescence correlation spectroscopy

Fluorescence lifetime

Single molecule spectroscopy

\begin{abstract}
A B S T R A C T
This chapter presents a concise introduction into the method of Fluorescence Lifetime Correlation Spectroscopy (FLCS). This is an extension of Fluorescence Correlation Spectroscopy (FCS) that analyses fluorescence intensity fluctuations from small detection volumes in samples of ultra-low concentration. FCS has been widely used for investigating diffusion, conformational changes, molecular binding/ unbinding equilibria, or chemical reaction kinetics, at single molecule sensitivity. In FCS, this is done by calculating intensity correlation curves for the measured intensity fluctuations. FLCS extends this idea by calculating fluorescence-lifetime specific intensity correlation curves. Thus, FLCS is the method of choice for all studies where a parameter of interest (conformational state, spatial position, molecular environmental condition) is connected with a change in the fluorescence lifetime. After presenting the theoretical and experimental basis of FLCS, the chapter gives an overview of its various applications. (c) 2018 The Authors. Published by Elsevier Inc. This is an open access article under the CC BY-NC-ND license
\end{abstract} (http://creativecommons.org/licenses/by-nc-nd/4.0/).

\section{Contents}

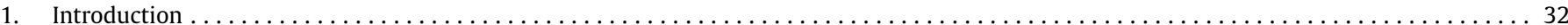

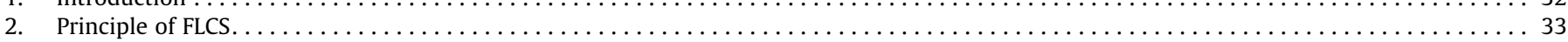

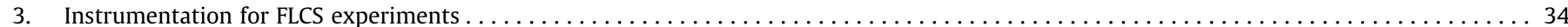

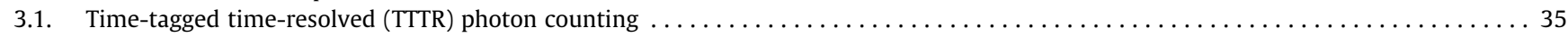

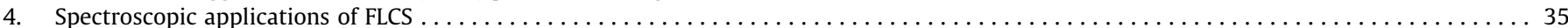

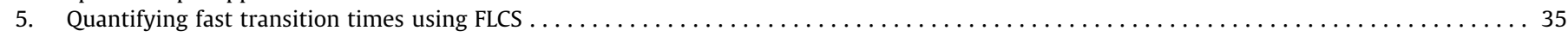

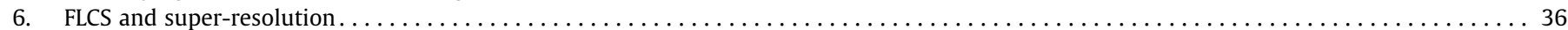

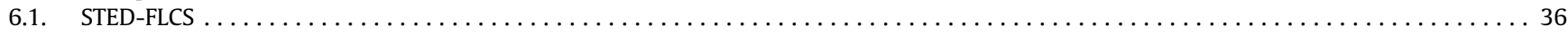

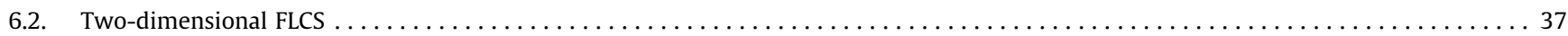

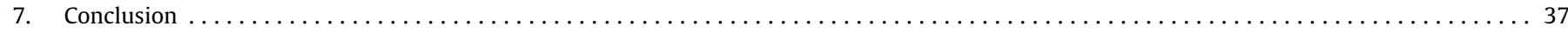

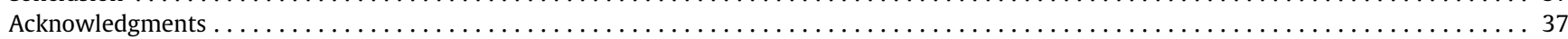

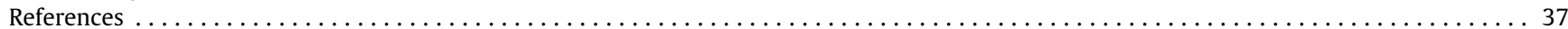

\section{Introduction}

Fluorescence Correlation Spectroscopy or FCS was first invented by Magde, Elson and Webb in the seventies of the last century [1-3]. Since then, it has become an indispensable tool for many applications in biology, biophysics, chemistry or physics [4-7]. In FCS, one excites and detects fluorescence emission of fluorescent or fluorescently labeled molecules out of a tiny detection volume (typically femtoliters), and applies a correlation analysis to the

\footnotetext{
* Corresponding author.

E-mail address: jenderl@gwdg.de (J. Enderlein).
}

recorded fluorescence intensity fluctuations. Any process that influences the measured fluorescence intensity (such as changes of emitter position due to diffusion, photophysical transitions, chemical reactions, or conformational changes) will show up as a prominent correlation decay in the correlation curve. For example, molecules that diffuse in and out of the detection volume will generate a stochastically fluctuating fluorescence signal, the correlation time of which is directly connected with their diffusion constant. Similarly, molecules that can switch into a nonfluorescent triplet state (intersystem crossing) will show a partial fluorescence correlation decay on the time scale of the intersystem crossing and triplet-to-ground state relaxation. 
Most of the applications of FCS are concerned with diffusion measurements. However, FCS can also be used to measure photophysical processes, molecular interactions, active transport etc. A special but powerful variant of FCS is Fluorescence CrossCorrelation Spectroscopy (FCCS) which measures the crosscorrelation of fluctuating fluorescence signals between spectrally different detection channels. This technique has been widely used to monitor the co-diffusion of different molecular species which are labeled with fluorescent dyes of different color [7-9].

However, cross-correlations cannot only be performed between fluorescent signals coming from different spectral detection windows, but also between fluorescent signals having different fluorescence lifetime signatures. This is the core idea of Fluorescence Lifetime Correlation Spectroscopy or FLCS, and was first demonstrated in [10]. Similar to dual-color FCCS, FLCS calculates autoand cross-correlation curves, but not in a spectrum-specific manner, but in a fluorescence-lifetime specific manner. The fundamental experimental requirement for FLCS is the ability to measure not only intensity fluctuations on a "long" time scale (microseconds to seconds), but to simultaneously monitor also the fluorescence decay on nanosecond time-scales. This is usually done by measuring fluorescence decay curves with Time-Correlated Single-Photon Counting (TCSPC) $[11,12]$, using pulsed excitation with high repetition rate $(\sim 10-100 \mathrm{MHz})$ and short ( $\sim 100 \mathrm{fs}-100 \mathrm{ps})$ laser pulses.

After the first publication of the concept of FLCS [10] (at that time called time-resolved FCS), it did not attract much attention, probably due to the exploding number of successful applications of conventional FCS at the time. FLCS was again picked up in 2005 [13], when it was used to efficiently eliminate effects of detector afterpulsing in FCS. In the same year, Benda et al. presented an upgraded lifetime-measuring confocal FCS system [14] that is ideally suited for performing FLCS. The term "FLCS" was then, for the first time, used in the following year in Refs. [15-17].

As already mentioned, FLCS is a cross-correlation spectroscopy technique that uses fluorescence lifetimes for calculating auto- and cross-correlations similar to conventional dual-color FCCS, which uses emission colors for discriminating between different fluorophores or fluorescent states. The fascinating property of FLCS is that one can distinguish fluorescence correlations of two or more emitting species that emit in the same spectral range, solely on the basis of their fluorescence lifetimes. In particular, the technique relies on the calculation of separate Auto-Correlation Functions (ACFs) for each emitting species by weighing the recorded photons with a filter function based on their emission delay with respect to the exciting laser pulses. These filter functions act as statistical filters which are calculated from "pure" decay patterns (TCSPC histograms) of each component. As such, FLCS can be applied in a similar way as is done with dual-color FCCS. However, its range of applications can be much wider: Recently, a conjunction of FLCS with STED has lead to a significant improvement in resolution, background suppression, and thus contrast in super-resolution fluorescence microscopy. A comprehensive introduction into FLCS and it's various applications until 2012 is nicely summarized in the review by Kapusta et al. [18]. Here, we provide an updated introduction into and review of FLCS which includes also its most recent applications and developments.

\section{Principle of FLCS}

An excellent introduction into the theoretical basis of FLCS has been given in [16], and we will briefly recapitulate it here. Let us consider a sample consisting of $m$ different species emitting fluorescence with different decay patterns such that the number of photons in the $i$ th TCSPC channel $(i=1, \ldots, L)$ at measurement time $t$ is
$I_{i}(t)=\sum_{\alpha=1}^{n} c_{\alpha}(t) p_{\alpha i}$

where $p_{\alpha i}$ is the discrete probability distribution function for detecting a photon in TCSPC-channel $i$ if the fluorescence comes from species $\alpha$, and $c_{\alpha}(t)$ is the momentary fluorescence intensity corresponding to species $\alpha$ at time $t$. We are interested in calculating fluorescence-decay specific two-photon auto- and crosscorrelation functions defined by

$g_{\alpha \beta}(t)=\left\langle c_{\alpha}\left(t_{0}\right) c_{\beta}\left(t+t_{0}\right)\right\rangle_{t_{0}}$

where $\alpha$ and $\beta$ can take values from 1 to $m$, and the angular brackets denote averaging over $t_{0}$. For that purpose, one has to extract the values $c_{\alpha}(t)$ from the measured photon stream. This is performed by calculating statistical filter functions $f_{\alpha i}$ such that they satisfy the relations

$\sum_{i} f_{\alpha i}\left\langle I_{i}(t)\right\rangle=\left\langle c_{\alpha}(t)\right\rangle$

and minimize the mean square errors

$\left\langle\left(\sum_{i} f_{\alpha i} I_{i}(t)-\left\langle c_{\alpha}(t)\right\rangle\right)^{2}\right\rangle$

where angular brackets denote time averaging, and the summations are performed over all TCSPC channels $i$. Using the fact that the photon detection in each TCSPC channel follows Poissonian statistics, these filter functions can be calculated using a weighted quasiinverse matrix operation [19] as

$\mathbf{f}=\left[\hat{\mathbf{M}} \cdot \operatorname{diag}\langle I\rangle^{-1} \cdot \hat{\mathbf{M}}^{T}\right]^{-1} \cdot \hat{\mathbf{M}} \cdot \operatorname{diag}\langle I\rangle^{-1}$.

Here, $\mathbf{f}$ is a matrix with elements $f_{\alpha i}, \widehat{\mathbf{M}}$ a matrix with elements $p_{\alpha i}$, and $\operatorname{diag}\langle I\rangle^{-1}$ is an $L \times L$-dimensional matrix with diagonal elements $\left\langle I_{j}\right\rangle^{-1}$. A big $T$ superscript denotes matrix transposition. An important point that should be emphasized here is that these filter functions form a dual orthogonal basis to the decay patterns. This means that element-wise multiplication and summation of these filter functions with the fluorescence decay patterns yields an identity matrix. Using these filters, the second order auto- and crosscorrelation function from Eq. (2) can now be expressed as

$g_{\alpha \beta}(t)=\sum_{j=1}^{L} \sum_{k=1}^{L} f_{\alpha j} f_{\beta k}\left\langle I_{j}\left(t+t_{0}\right) I_{k}\left(t_{0}\right)\right\rangle_{t_{0}}$

For $\alpha=\beta$, we obtain lifetime auto-correlations which represent the intensity fluctuations of each individual lifetime species, and for $\alpha \neq \beta$, we obtain the cross-correlations of $\alpha$ versus $\beta$, which indicate the probability of detecting a second photon from species $\beta$ at time $t$ after detecting a photon from species $\alpha$ at time zero. Thus, for a sample comprising of two molecular species with two distinct fluorescence decay patterns, one obtains two lifetime autocorrelation curves, one for each pattern, and two cross-correlation curves for correlations between the patterns. Generally, the crosscorrelations are not symmetric, $g_{\alpha \beta} \neq g_{\beta \alpha}$. Equality occurs only under strict conditions such as pure co-diffusion, but if one considers e.g. transitions between two states that are part of an interconnected multi-state system containing more than only these two states, the observed cross-correlations between the two states may be asymmetric.

It is important to emphasize that throughout the above described mathematics, we did not put any restriction on the nature of $p_{\alpha i}$. Therefore, one can apply FLCS to fluorescent samples exhibiting any form of fluorescence decay kinetics (singleexponential, multi-exponential, etc.). A straightforward extension of the above concept is to include an additional component with 


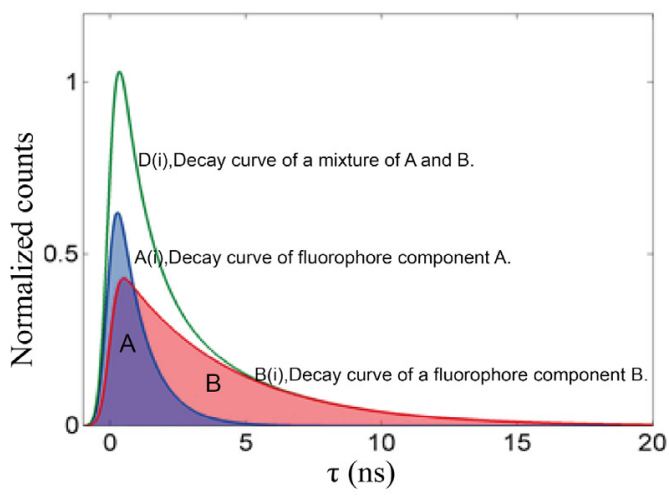

(a)

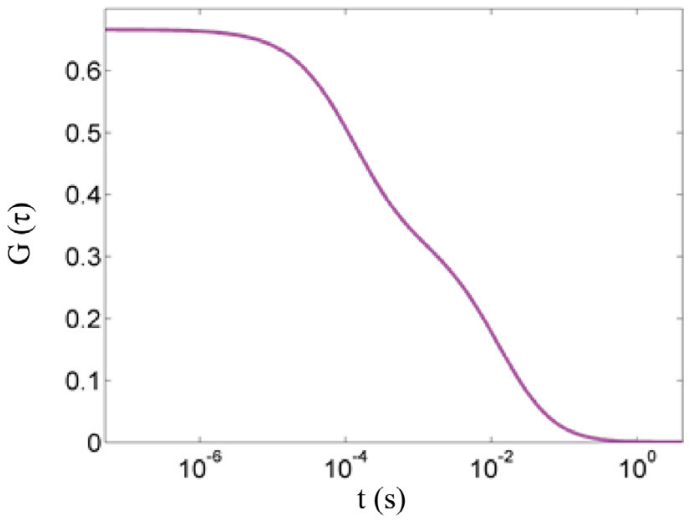

(c)

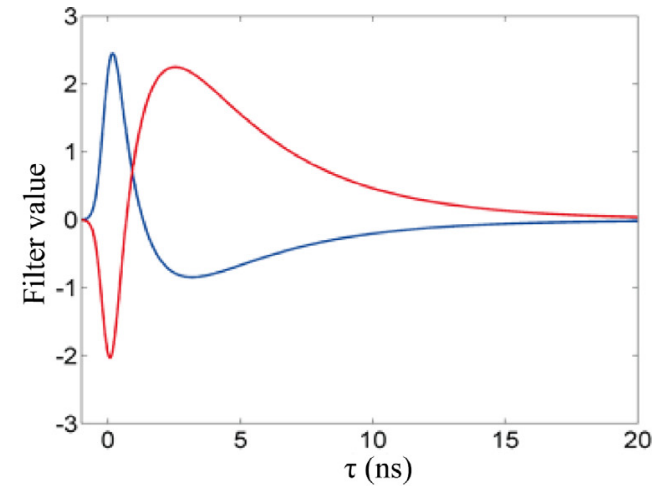

(b)

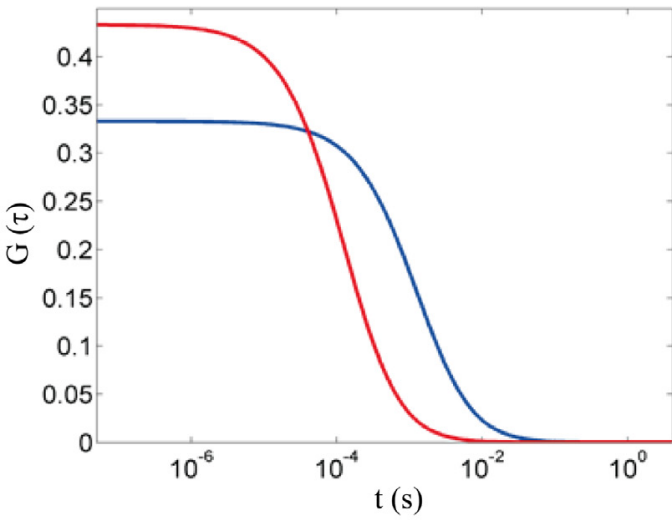

(d)

Fig. 1. Working principle of FLCS. (a) Total TCSPC curve of a mixture of species A and B (green), and individual TCSPC curves of each component A (blue) and B (red). (b) Lifetime-specific filter functions calculated from the component-specific TCSPC histograms shown in (a). Note that the filter function is positive for the shorter lifetime component for the first few nanoseconds, while it is positive for the longer component at longer decay times. (c) ACF obtained with conventional FCS containing contributions from both the components A and B. (d) Separate ACFs calculated for each component A and B by weighing the photons based on their arrival times with the filter functions shown in (b). (For interpretation of the references to colour in this figure caption, the reader is referred to the web version of this article.)

a uniform probability distribution across the TCSPC channels in order to eliminate background contributions (e.g. dark counts, detector afterpulsing) from the final fluorescence auto- and cross-correlations. The presented theory is generic and has been applied in a variety of works other than FLCS such as fluorescence spectral correlation spectroscopy [20], or filtered FCS [21] (Fig. 1).

\section{Instrumentation for FLCS experiments}

FLCS experiments are usually performed with a confocal microscope equipped with fluorescence lifetime and FCS measurement capability (e.g. MicroTime 200, PicoQuant GmbH, Berlin, Germany). Fig. 2 shows a schematic of such a setup. In the following, we will give the details of our existing MicroTime 200 system which is routinely used for FLCS measurements. A linearly polarized pulsed diode laser ( $\lambda_{\text {exc }}=485 \mathrm{~nm}$, pulse duration $100 \mathrm{ps}$ FWHM, LDH-P-C-485B, PicoQuant) equipped with a clean-up filter (BrightLine FF01-480/17, Semrock) is passed through a quarterwave plate (AQWP05M-600, Thorlabs GmbH, Dachau/Munich, Germany) to generate a circularly polarized laser beam. The laser is pulsed at a repetition rate of $40 \mathrm{MHz}$ by using a multichannel picosecond laser driver (PDL 828 "Sepia II", PicoQuant). This beam is then coupled into a polarization-maintaining single-mode fiber (PMC-400-4.2-NA010-3-APC- 250 V, Schäfter und Kirchhoff GmbH, Hamburg, Germany). At the fiber output, the light is collimated and reflected by a dichroic mirror (FITC/TRITC Chroma Technology, Rockingham, VT, USA) into the microscope's objective (UPLSAPO $100 \times$ oil, 1.2 N.A., Olympus Deutschland, Hamburg, Germany). The same water immersion objective is used for collecting the

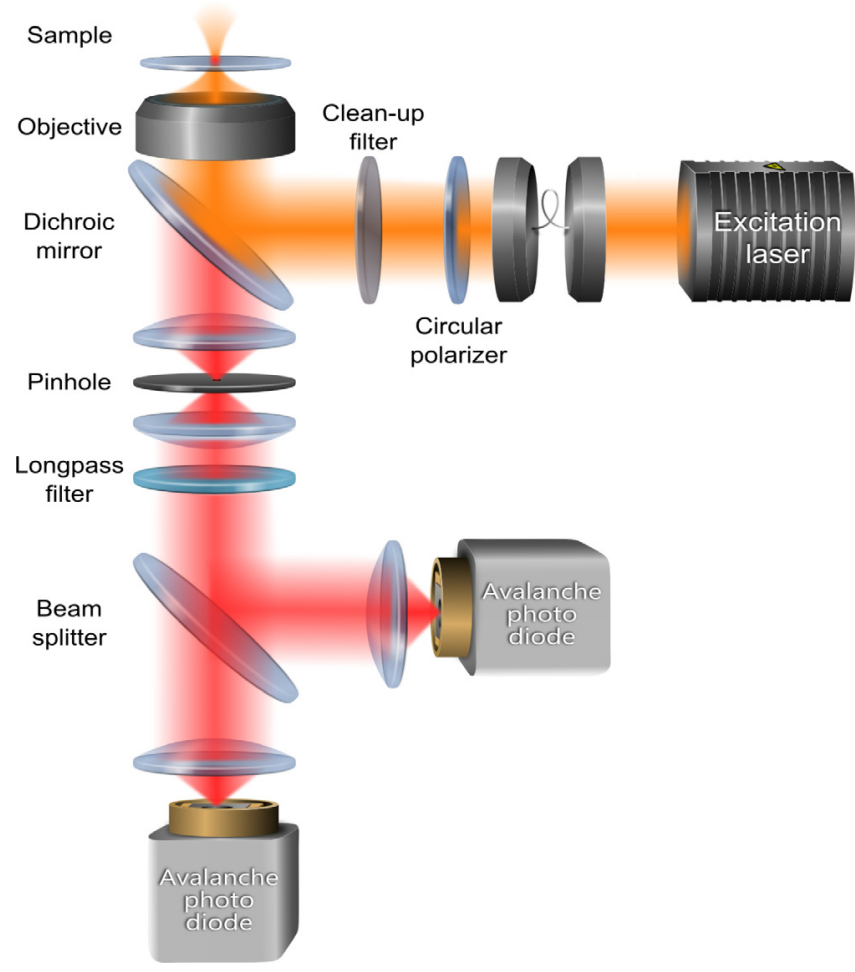

Fig. 2. Schematic of FLCS setup. For explanation see main text. 
fluorescence from the sample. Back-scattered light from the laser is blocked using a longpass filter (BLP01-488R-25, Semrock). After passing through a $100 \mu \mathrm{m}$ pinhole, the fluorescence beam is collimated again and focused onto two single-photon avalanche diodes (SPCM-CD 3516 H, Excelitas Technologies GmbH \& Co. KG, Wiesbaden, Germany). A multichannel picosecond event timer (HydraHarp 400, PicoQuant) is used to record the detected photons from both detectors independently with an absolute temporal resolution of $2 \mathrm{ps}$ on a common time frame. For measurements in solution, a droplet $(\sim 30 \mu \mathrm{L})$ of sample is placed on top of a clean glass coverslip, and the objective is focused approximately $30 \mu \mathrm{m}$ into the solution.

\subsection{Time-tagged time-resolved (TTTR) photon counting}

In our FLCS measurements, a HydraHarp 400 (PicoQuant, Berlin) event timer electronics is used for recording single photon detection events. A detailed description of this instrumentation and its working can be found in Ref. [22] (see also [23,24]). Briefly, the event timing module has the capability to process up to 64 input channels, while using one channel for synchronization with the laser pulses. Each input channel is processed by a separate timing circuit, called a time-to-digital converter (TDC), while all of these TDCs are connected to a single internal master clock. The internal clock serves as the time base for all the individual timers of each detection channel and synchronizes their operation. Picosecond timing resolution for each channel is achieved by interpolation based on a phase-locked loop system in each TDC [25]. In this way, each event in all channels can be timed with a few picoseconds resolution. In the end, each detected photon is assigned two time tags, one with respect to the last laser pulse sync, called the micro-time $\tau$, and the other with respect to the start of the experiment, called the macro-time $t$ which is usually counted as the number of syncs preceding the detection event [22] (see Fig. 3). Together with these time-tags, also the detection channel is recorded for each photon. Using the micro-times, one can calculate the TCSPC histogram for each detector channel, whereas from the macro-times of the detected photons, correlation curves are calculated, using a dedicated correlation algorithm for asynchronous photon detection data [26].

\section{Spectroscopic applications of FLCS}

There are two core advantages of FLCS over dual-color FCS and FCCS: (a) absence of spectral cross-talk and (b) absence of artefacts arising from a non-perfect overlap of detection volumes. Naturally, FLCS attracted early applications analogous to dual-color FCCS for investigating bio-molecular interactions. One such example is the quantification of protein-protein (EGFP-mCherry) interaction in live cells by Padillapara et al. [27]. In their work, they simultaneously applied pulsed excitation for GFP along with continuous

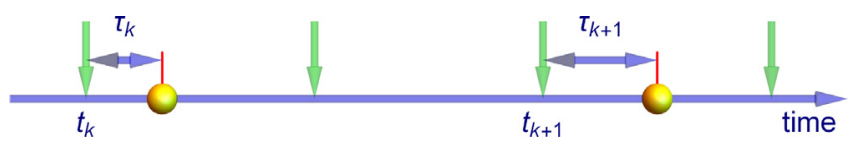

Fig. 3. TTTR counting scheme: single-photon detection events are timed on two different time scales. On a pico- to nanosecond time-scale, the so-called micro-time $\left(\tau_{k}\right)$ records the detection time of each photon $k$ (yellow balls) with respect to the last preceding laser pulse (green arrows). If one histograms these micro-times, one obtains the TCSPC fluorescence decay histogram, the bin width of which is determined by the discrete temporal resolution of the micro-times. On a time scale slower than the laser pulse repetition period, absolute arrival times of photons are recorded with a temporal resolution of one laser period (macro-time $t_{k}$ ). From both time tags, the absolute detection time with an overall temporal resolution of picoseconds can be reconstructed. (For interpretation of the references to colour in this figure caption, the reader is referred to the web version of this article.) wave (cw) excitation for mCherry, and FLCS filtering was used to eliminate spectral cross-talk between the two spectral detection channels. A similar live-cell study used FLCS for obtaining crosscorrelation curves for GFP and Alexa-488 labeled molecules [28]. A number of other works used the unique capability of FLCS of obtaining lifetime-specific ACFs for watching molecular state transitions upon changes of the molecular environment. One such study looked at the fluorescence lifetime reduction of a fluorophore close to the surface of silver nanoparticles [29]. In particular, the authors used FLCS for probing metal-fluorophore interactions in solution at the single-molecule level. For doing this, ss-oligonucleotides were covalently bound to silver nanoparticles and then hybridized with complementary strands labeled with Cy5. This resulted in substantial reduction of the dye's lifetime in the bound state. In another study, Benda et al. reported FLCS measurements in conjunction with lifetime tuning [15] to study molecular diffusion in Supported Lipid Bilayers (SLBs), while having a considerable amount of unbound dye in the aqueous phase above the bilayer. The authors used Indium Tin Oxide (ITO) covered glass slides as the bilayer support, using the fact that ITO shows similar distance dependent quenching (and thus lifetime-reducing) properties as metals. Thus, calculating FCS curves in a lifetimespecific manner, they were able to successfully separate correlation contributions stemming from free dyes in solution from correlation contributions of bilayer-associated molecules. In a series of other studies, FLCS has been extensively used for suppressing ACF contributions from scattered light or other background sources such as detector afterpulsing and electronics dark counts $[13,17,30]$. This is particularly important when using FCS for estimating local molecular concentrations, where any unaccounted background contribution in the FCS curve will lead to gross overestimates of the actual concentration. In this context, FLCS has been widely used for background suppression without explicit reference to the technique itself [31-33].

Application of FLCS in biology was first reported in 2008 (see [34-36]) where the method was utilized for gaining detailed insight into DNA condensation by spermine, a cationic compound. This is an important topic in the context of improving non-viral gene therapy via delivery of large DNA molecules across biomembranes. In their study, the intercalating dye Picogreen (Invitrogen, Carlsbad, CA, US) was used for labeling DNA, and it's lifetime change during DNA condensation (from $\sim 4.5 \mathrm{~ns}$ in the extended state to $\sim 3.5 \mathrm{~ns}$ in the condensed state) was used to calculate ACFs for the different DNA condensation states. The cross-correlation functions (CCFs) between the two states at spermin titration midpoint revealed a dynamic switching between the extended and condensed phase on a millisecond timescale. This work demonstrated for the first time the usefulness of FLCS in cases where the transition rate between states with different fluorescence lifetime is too fast for being captured in a conventional TCSPC-based lifetime measurement. Additionally, this study demonstrated the possibility of using lifetime fluctuations as a reporter for fast alterations in the local environment. Paredes et al. probed protontransfer reactions of Tokyo-Green-II (TG-II) under varying local environments [37,38], and the same group used TG-II also to study reverse micelles of the surfactant sodium bis(2-ethylhexyl) sulfosuccinate in organic solvents [39]. In another publication, FLCS together with Förster Resonance Energy Transfer (FRET) was exploited for extracting kinetic rates of the inter-conversion of Syntaxin 1 between it's two conformers [21].

\section{Quantifying fast transition times using FLCS}

One of the most exciting applications of FLCS is to determine fast transition rates between states of different fluorescent lifetimes in a molecule. As an example, we recently studied with FLCS 


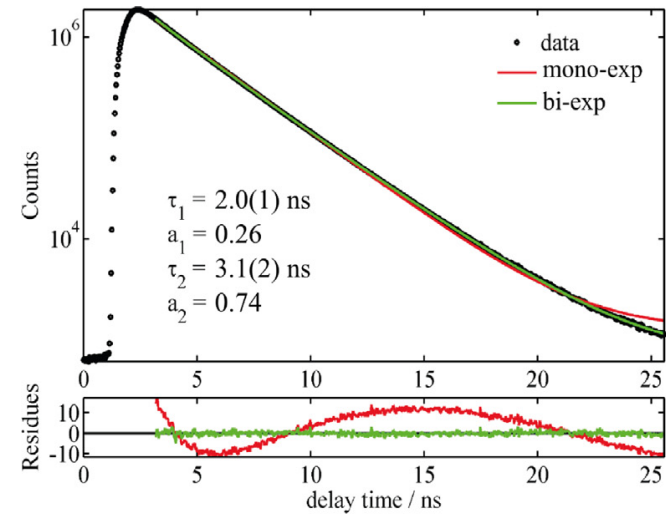

(a)

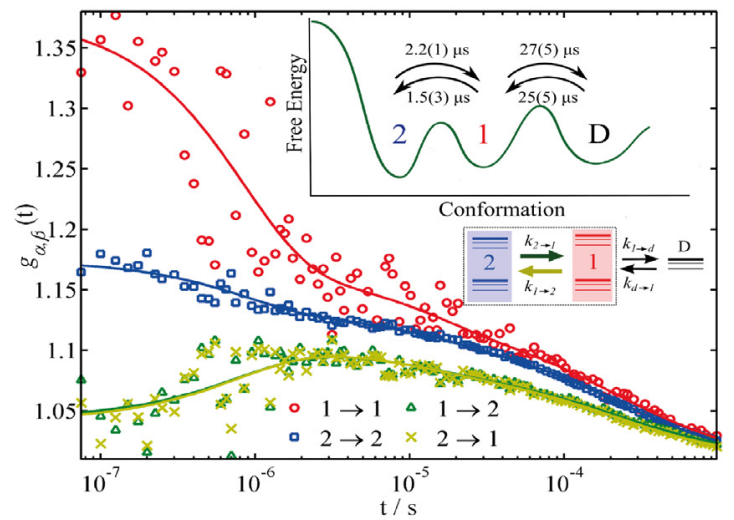

(b)

Fig. 4. (a) TCSPC histogram of EGFP fluorescence decay. The data was fitted using a mono-exponential (red) and a bi-exponential decay model (green). As can be clearly seen from the residues, the data can only be satisfactorily fitted using the bi-exponential model. The associated two decay times are $2.0(1)$ ns and $3.1(2)$ ns with amplitudes of 0.26 and 0.74 , respectively. (b) FLCS auto- and cross-correlation curves for EGFP at pH 6.0 at an excitation power of $\sim 200 \mathrm{~kW} / \mathrm{cm}^{-2}$. $1 \rightarrow 1$ and $2 \rightarrow 2$ denote the auto-correlations corresponding to state 1 and 2 with $\tau_{1}=2.0(1)$ ns and $\tau_{2}=3.1(2)$ ns, respectively; whereas $1 \rightarrow 2$ and $2 \rightarrow 1$ represent the cross-correlations between the two states. The curves are normalized with respect to their amplitudes at time $0.1 \mathrm{~s}$ when all the correlations have decayed completely. Solid lines are fits of the three-state model which is schematically shown in the inset, which also displays the fitted transition rate values (figure reprinted from Ref. [40] with permission of publisher). (For interpretation of the references to colour in this figure caption, the reader is referred to the web version of this article.)

the complex photophysics of the fluorescent protein EGFP [40]. The chromophore of EGFP exhibits two fluorescent states with lifetimes of 2.0(1) ns and 3.1(2) ns (Fig. 4(a)). Thus, we used FLCS for determining the transition rates between these two states, which occur to be on the microsecond time scale. The transition rates were found to be independent of $\mathrm{pH}$ or excitation power, and we could relate them to an Ångström-scale rotational isomerization of a glutamic acid residue (E222) that is adjacent to the fluorescent center. It should be noted that the excitation and emission spectra of the two fluorescent states are highly overlapping and basically inseparable at room temperature, so that the transition between them can only be resolved by using FLCS. Moreover, this fast transition between the two fluorescent states is coupled to a further transition between one of them and a dark state, which takes place on a much slower timescale. This bright-to-dark state transition is well-known to be associated with a protonation and deprotonation of the chromophore [41]. Thus, the full chromophore photophysics of EGFP is described by a three-state coupled reaction system in which the dark state can only be reached via an intermediate fluorescent state, see inset in Fig. 4(b). This is very similar to what has been observed earlier in wild-type GFP [42-45]. However, FLCS finds faster switching rates compared to those found by conventional FCS. This discrepancy is related to the two non-zero eigenvalues of the three-state consecutive reaction model. Denoting the bright states as $\mathbf{1}$ and $\mathbf{2}$ and the dark state as $\mathbf{D}$, and by introducing the relations $k_{\text {fast }}=k_{1 \rightarrow 2}+k_{2 \rightarrow 1}$ and $k_{\text {slow }}=k_{1 \rightarrow \mathrm{D}}+k_{\mathrm{D} \rightarrow 1}$, (with the simplifying assumptions that $k_{1 \rightarrow 2} \approx k_{2 \rightarrow 1}, k_{1 \rightarrow \mathrm{D}} \approx k_{\mathrm{D} \rightarrow 1}$ ), these eigenvalues can be written as

$\lambda_{2,3}=-\frac{1}{2}\left(k_{\text {fast }}+k_{\text {slow }}\right)\left[1 \mp \sqrt{1-\frac{3 k_{\text {fast }} k_{\text {slow }}}{\left(k_{\text {fast }}+k_{\text {slow }}\right)^{2}}}\right]$

For the case that the fast transition rate, $k_{\text {fast }}$, is significantly larger than the slow transition rate, $k_{\text {slow }}$, this expression leads to the following approximate eigenvalues

$\lambda_{2} \approx-k_{\text {fast }}, \quad \lambda_{3} \approx-\frac{3}{4} k_{\text {slow }}$

Thus, a conventional FCS experiment will also observe a fast and a slow transition rate, but it will underestimate the slow rate by $\sim 25 \%$ ! This discrepancy becomes even more pronounced if the protonation is faster than the deprotonation $\left(k_{1 \rightarrow \mathrm{D}}>k_{\mathrm{D} \rightarrow 1}\right)$, i.e. for $\mathrm{pH}$

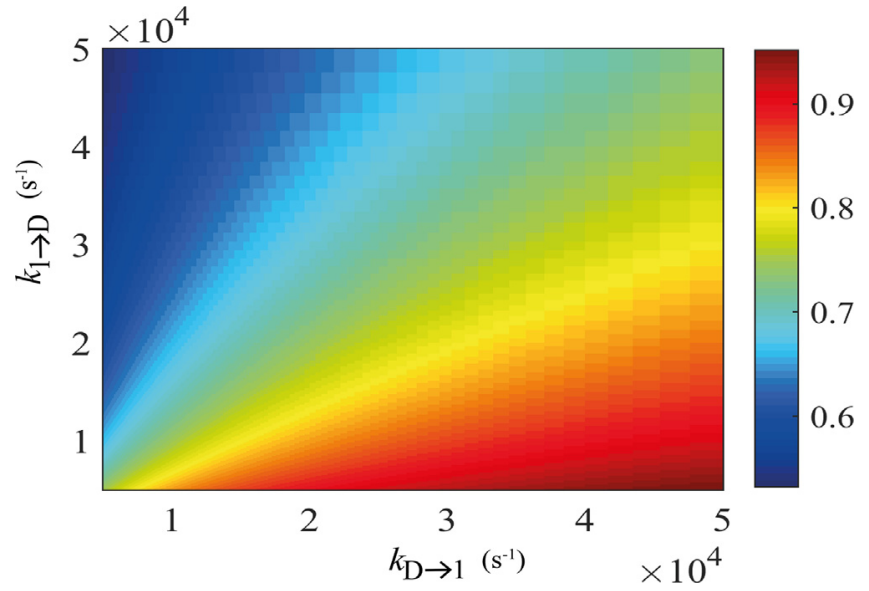

Fig. 5. The ratio of the relaxation rate between the states $\mathbf{1}$ and $\mathbf{D}$ estimated by FLCS (using a three-state-consecutive reversible reaction model) versus FCS as a function of the forward $k_{\mathrm{D} \rightarrow 1}$ and backward $k_{1 \rightarrow \mathrm{D}}$ rates is shown. When $k_{\mathrm{D} \rightarrow 1}=k_{1 \rightarrow \mathrm{D}}$, conventional FCS underestimates the relaxation rate by $25 \%$.

values lower than the pKa of EGFP ( 5.7) [46]. Fig. 5 shows a detailed map of the difference in fast-rate determination between conventional FCS and FLCS (which always yields the correct value) as a function of the slow rates $k_{1 \rightarrow \mathrm{D}}$ and $k_{\mathrm{D} \rightarrow 1}$.

\section{FLCS and super-resolution}

\subsection{STED-FLCS}

In Ref. [47], FLCS was combined with STED microscopy to improve imaging contrast and resolution. The authors applied it to unveil the spatio-temporal heterogeneity of molecular diffusion of fluorescent lipid analogues in membranes of mammalian cells. FLCS-STED is an improved version of the combination of gateddetection STED (gSTED) [48] with FCS. The core idea is that the STED laser induces a gradual de-excitation of excited molecules from low to high intensity regions in the STED intensity distribution, which gradually shortens the excited state lifetime in a position-dependent manner. Thus, different exponential 
components of the observed fluorescence decay curve correspond to different positions of a fluorophore within the STED intensity distribution. By filtering out different exponential decay components within the observed fluorescence lifetime decay, one selects different spatial positions within the excitation/detection volume. In gSTED, this is done by selecting photons within a small time interval (time gate), whereas FLCS filters photons for a specific decay time. In this way, one can tune the detection volume/area from which fluorescence is efficiently detected, and thus tune the spatial resolution. In [49], the authors call this method also Separation of Photons by LIfetime Tuning or SPLIT. They have applied SPLIT-FLCS to probe the three-dimensional diffusion of Enhanced Green Fluorescent Protein (EGFP) at a spatial resolution of $\sim 80$ $\mathrm{nm}$ [50]. The study reveals the spatial inhomogeneity in EGFP and tubulin-EGFP diffusion in the cytoplasm of living cells.

\subsection{Two-dimensional FLCS}

An interesting extension of the concept of FLCS was presented by Tahara and colleagues [51,52]. Termed as Two-dimensional FLCS (2dFLCS), the method generates a two-dimensional photon pair correlation plot $g\left(t, \tau_{1}, \tau_{2}\right)$ which measures the timecorrelation (lag time $t$ ) of photons with fluorescence decay time $\tau_{1}$ against photons with fluorescence decay time $\tau_{2}$. It is thus a continuum extension of the original idea of FLCS, which was initially worked out for correlating photon pairs belonging to discrete sets of fluorescence decay patterns. The hardware requirements of 2dFLCS are identical to those of conventional FLCS, but data analysis is much more involved. From the raw photon arrival (TTTR) data, one calculates a three-dimensional correlogram, where the first two dimensions correspond to the TCSPC detection channels of the first and second photon, and the third dimension is the correlation lag time. Then, by using a regularized inverse Laplace transform, one obtains the desired 2dFLCS correlogram which shows the correlation between photons belonging to different fluorescence decay times. For the theoretical details, see [51]. The core advantage of this approach is that it does not require any prior knowledge of the expected fluorescence decay patterns of a sample. The challenge of $2 \mathrm{dFLCS}$ is that due to correlating photons over a two-dimensional lifetime continuum instead of using a discrete set of lifetime patterns, one needs exceptionally good signal-tonoise ration and thus large measurement times. Moreover, the ill-defined inverse Laplace transform may be numerically unstable and requires some skills for finding the best regularization.

2dFLCS has been successfully applied to probe the equilibrium conformational and reaction dynamics in complex biomolecules with single-molecule sensitivity, in particular fast ( $\mu$ s time range) loop formation kinetics in DNA hairpins [52]. In these studies, DNA hairpin molecules were labeled with a FRET pair, and 2dFLCS was used to generate 2D correlation maps of the donor lifetime. This correlation map was then used to analyze the hairpin dynamics. In another study, 2dFLCS was applied to address the prototypical question of the conformation-function relationship of proteins [53]. In particular, this study investigated the conformational dynamics of cytochrome $c$ and revealed a microsecond transition kinetics between different conformers, and thus elucidated the free energy landscape of protein folding [53].

\section{Conclusion}

As we have shown, FLCS can be a useful extension of FCS for all cases where a state or process of interest is connected with a characteristic fluorescence lifetime or lifetime change, respectively. In particular, the cross-correlation between different lifetime states can provide valuable information about rapid (sub-microsecond to millisecond) transitions between these states which are usually difficult to access by other methods. This can be particularly useful in complex luminescent systems where a multi-state reaction kinetics is observed. As an example, many intrinsically fluorescent proteins exhibit bi- or multi-exponential fluorescence decay patterns [55-57]. Such a multi-exponential decay pattern is often due to fast inter-conversion between states having monoexponential lifetimes. However, such transitions cannot be disentangled by using conventional FCS, which can see the overall intensity fluctuations connected with these transitions, but cannot resolve the transition kinetics between them. Here, FLCS can be the method of choice for resolving individual state transitions and for measuring their transition rates as was shown here for the photophysical three-state system in EGFP (Section 5).

In a similar vain, we see a huge potential of FLCS for the investigation of fast conformational dynamics in biomolecules such as intrinsically disordered or unfolded proteins [59-60]. When applying single-molecule fluorescence spectroscopy, the method of choice is usually single-molecule FRET (smFRET) [61,62] in conjunction with advanced fluctuation analysis [63-67]. Here, FLCS can be a valuable complementary method by quantifying correlations of the donor's lifetime, especially since the theoretical details for this application are well worked out [68]. A first proof-ofprinciple of this idea was presented in [16], where the authors used FLCS to resolve and quantify the rapid transition between two conformational states in a dye-protein construct.

Another emerging and fascinating application of FLCS is its combination with the recently developed technique of MetalInduced Energy Transfer (MIET) [69]. In MIET, one uses the effect that the fluorescence lifetime and intensity dramatically change if an emitter is brought close to a metal, due to energy transfer form the emitter's excited state to metal plasmons (similar to the quenching of acceptor fluorescence by a donor in FRET). This can be i.a. used to measure the distance of a fluorescent emitter from a metallized surface with nanometer accuracy [70,71]. In conjunction with FLCS, MIET can be used for investigating rapid molecular dynamics that is connected with a positional change of a fluorescence label with respect to a fixed metallized surface. The advantage of such a FLCS-MIET approach, as compared to conventional SMFRET, would be that it requires only the specific fluorescent labeling of one site on the molecule of interest (instead of two), and that it avoids the numerous technical complications usually associated with precisely quantifying smFRET measurements [72].

In conclusion, it can be expected that FLCS will find broad application in all cases where systems or processes of interest are reported by specific fluorescence lifetime patterns or fluorescence lifetime changes, and where one is interested in single-molecule sensitivity.

\section{Acknowledgments}

We thank the Deutsche Forschungsgemeinschaft (DFG) for its financial support (project A06 of SFB860, and project A07 of SFB937).

\section{References}

[1] D. Magde, E. Elson, W.W. Webb, Thermodynamic fluctuations in a reacting system-measurement by fluorescence correlation spectroscopy, Phys. Rev. Lett. 29 (11) (1972) 705.

[2] E.L. Elson, D. Magde, Fluorescence correlation spectroscopy. I. Conceptual basis and theory, Biopolymers 13 (1) (1974) 1-27.

[3] D. Magde, E.L. Elson, W.W. Webb, Fluorescence correlation spectroscopy. II. An experimental realization, Biopolymers 13 (1) (1974) 29-61.

[4] N.L. Thompson, Fluorescence correlation spectroscopy, in: Topics in Fluorescence Spectroscopy, Springer, 2002, pp. 337-378.

[5] O. Krichevsky, G. Bonnet, Fluorescence correlation spectroscopy: the technique and its applications, Rep. Prog. Phys. 65 (2) (2002) 251 
[6] P. Schwille, Fluorescence correlation spectroscopy, in: Encyclopedic Reference of Genomics and Proteomics in Molecular Medicine, Springer, 2005, pp. 576578 .

[7] R. Rigler, E.S. Elson, Fluorescence correlation spectroscopy: theory and applications, vol. 65, Springer Science \& Business Media, 2012

[8] E. Petrov, P. Schwille, State of the art and novel trends in fluorescence correlation spectroscopy, in: Standardization and Quality Assurance in Fluorescence Measurements II, Springer, 2008, pp. 145-197.

[9] B. Valeur, J.-C. Brochon, New Trends in Fluorescence Spectroscopy: Applications to Chemical and Life Sciences, vol. 1, Springer Science \& Business Media, 2012

[10] M. Böhmer, M. Wahl, H.-J. Rahn, R. Erdmann, J. Enderlein, Time-resolved fluorescence correlation spectroscopy, Chem. Phys. Lett. 353 (5) (2002) 439445.

[11] W. Becker, Advanced Time-correlated Single Photon Counting Techniques, vol. 81, Springer Science \& Business Media, 2005.

[12] D. O'Connor, Time-correlated Single Photon Counting, Academic Press, 2012.

[13] J. Enderlein, I. Gregor, Using fluorescence lifetime for discriminating detector afterpulsing in fluorescence-correlation spectroscopy, Rev. Sci. Instrum. 76 (3) (2005). 033102.

[14] A. Benda, M. Hof, M. Wahl, M. Patting, R. Erdmann, P. Kapusta, TCSPC upgrade of a confocal FCS microscope, Rev. Sci. Instrum. 76 (3) (2005). 033106.

[15] A. Benda, V. Fagul'ová, A. Deyneka, J. Enderlein, M. Hof, Fluorescence lifetime correlation spectroscopy combined with lifetime tuning: new perspectives in supported phospholipid bilayer research, Langmuir 22 (23) (2006) 9580-9585.

[16] I. Gregor, J. Enderlein, Time-resolved methods in biophysics. 3. Fluorescence lifetime correlation spectroscopy, Photochem. Photobiol. Sci. 6 (1) (2007) 13 18.

[17] P. Kapusta, M. Wahl, A. Benda, M. Hof, J. Enderlein, Fluorescence lifetime correlation spectroscopy, J. Fluoresc. 17 (1) (2007) 43-48.

[18] P. Kapusta, R. Macháň, A. Benda, M. Hof, Fluorescence lifetime correlation spectroscopy (FLCS): concepts, applications and outlook, Int. J. Mol. Sci. 13 (10) (2012) 12890-12910.

[19] J. Enderlein, R. Erdmann, Fast fitting of multi-exponential decay curves, Opt. Commun. 134 (1) (1997) 371-378.

[20] A. Benda, P. Kapusta, M. Hof, K. Gaus, Fluorescence spectral correlation spectroscopy (FSCS) for probes with highly overlapping emission spectra, Opt. Express 22 (3) (2014) 2973-2988.

[21] S. Felekyan, S. Kalinin, H. Sanabria, A. Valeri, C.A. Seidel, Filtered FCS: species auto-and cross-correlation functions highlight binding and dynamics in biomolecules, ChemPhysChem 13 (4) (2012) 1036-1053.

[22] M. Wahl, H.-J. Rahn, I. Gregor, R. Erdmann, J. Enderlein, Dead-time optimized time-correlated photon counting instrument with synchronized, independent timing channels, Rev. Sci. Instrum. 78 (3) (2007). 033106.

[23] M. Wahl, T. Röhlicke, H.-J. Rahn, R. Erdmann, G. Kell, A. Ahlrichs, M. Kernbach, A.W. Schell, O. Benson, Integrated multichannel photon timing instrument with very short dead time and high throughput, Rev. Sci. Instrum. 84 (4) (2013). 043102.

[24] M. Wahl, H.-J. Rahn, T. Röhlicke, G. Kell, D. Nettels, F. Hillger, B. Schuler, R. Erdmann, Scalable time-correlated photon counting system with multiple independent input channels, Rev. Sci. Instrum. 79 (12) (2008) 123113.

[25] J. Kalisz, Review of methods for time interval measurements with picosecond resolution, Metrologia 41 (1) (2003) 17.

[26] M. Wahl, I. Gregor, M. Patting, J. Enderlein, Fast calculation of fluorescence correlation data with asynchronous time-correlated single-photon counting, Opt. Express 11 (26) (2003) 3583-3591.

[27] S. Padilla-Parra, N. Audugé, M. Coppey-Moisan, M. Tramier, Dual-colo fluorescence lifetime correlation spectroscopy to quantify protein-protein interactions in live cell, Microsc. Res. Tech. 74 (8) (2011) 788-793.

[28] J. Chen, J. Irudayaraj, Fluorescence lifetime cross correlation spectroscopy resolves egfr and antagonist interaction in live cells, Anal. Chem. 82 (15) (2010) 6415-6421.

[29] K. Ray, J. Zhang, J.R. Lakowicz, Fluorescence lifetime correlation spectroscopic study of fluorophore-labeled silver nanoparticles, Anal. Chem. 80 (19) (2008) 7313-7318.

[30] S. Rüttinger, P. Kapusta, M. Patting M. Wahl, R. Macdonald, On the resolution capabilities and limits of fluorescence lifetime correlation spectroscopy (FLCS) measurements, J. Fluoresc. 20 (1) (2010) 105-114.

[31] R. Macháň, M. Hof, T. Chernovets, M.N. Zhmak, T.V. Ovchinnikova, J. Sýkora Formation of arenicin-1 microdomains in bilayers and their specific lipid interaction revealed by z-scan FCS, Anal. Bioanal. Chem. 399 (10)(2011) 3547 3554 .

[32] R. Macháň, A. Miszta, W. Hermens, M. Hof, Real-time monitoring of melittininduced pore and tubule formation from supported lipid bilayers and its physiological relevance, Chem. Phys. Lipids 163 (2) (2010) 200-206.

[33] G. Pembouong, N. Morellet, T. Kral, M. Hof, D. Scherman, M.-F. Bureau, N. Mignet, A comprehensive study in triblock copolymer membrane interaction, J. Controlled Release 151 (1) (2011) 57-64.

[34] J. Humpolíčková, L. Beranová, M. Štěpánek, A. Benda, K. Procházka, M. Hof, Fluorescence lifetime correlation spectroscopy reveals compaction mechanism of 10 and $49 \mathrm{kbp}$ DNA and differences between polycation and cationic surfactant, J. Phys. Chem. B 112 (51) (2008) 16823-16829.

[35] J. Humpolíčková, A. Benda, J. Sýkora, R. Macháň, T. Kral, B. Gasinska, J. Enderlein, M. Hof, Equilibrium dynamics of spermine-induced plasmid DNA condensation revealed by fluorescence lifetime correlation spectroscopy, Biophys. J. 94 (3) (2008) L17-L19.
[36] J. Humpolíčková, M. Štěpánek, T. Kral, A. Benda, K. Procházka, M. Hof, On mechanism of intermediate-sized circular DNA compaction mediated by spermine: contribution of fluorescence lifetime correlation spectroscopy, J. Fluoresc. 18 (3-4) (2008) 679-684.

[37] J.M. Paredes, L. Crovetto, A. Orte, J.M. Alvarez-Pez, E.M. Talavera, Influence of the solvent on the ground-and excited-state buffer-mediated proton-transfer reactions of a xanthenic dye, РCCP 13 (4) (2011) 1685-1694.

[38] J.M. Paredes, L. Crovetto, A. Orte, S.G. Lopez, E.M. Talavera, J.M. Alvarez-Pez, Photophysics of the interaction between a fluorescein derivative and ficoll, J. Phys. Chem. A 115 (46) (2011) 13242-13250.

[39] J.M. Paredes, A. Garzon, L. Crovetto, A. Orte, S.G. Lopez, J.M. Alvarez-Pez, Effects of the anion salt nature on the rate constants of the aqueous proton exchange reactions, PCCP 14 (16) (2012) 5795-5800.

[40] A. Ghosh, S. Isbaner, M. Veiga-Gutiérrez, I. Gregor, J. Enderlein, N. Karedla, Quantifying microsecond transition times using fluorescence lifetime correlation spectroscopy, J. Phys. Chem. Lett. 8 (24) (2017) 6022-6028.

[41] U. Haupts, S. Maiti, P. Schwille, W.W. Webb, Dynamics of fluorescence fluctuations in green fluorescent protein observed by fluorescence correlation spectroscopy, Proc. Nat. Acad. Sci. 95 (23) (1998) 13573-13578.

[42] K. Brejc, T.K. Sixma, P.A. Kitts, S.R. Kain, R.Y. Tsien, M. Ormö, S.J. Remington, Structural basis for dual excitation and photoisomerization of the Aequorea victoria green fluorescent protein, Proc. Nat. Acad. Sci. 94 (6) (1997) 2306 2311.

[43] G.J. Palm, A. Zdanov, G.A. Gaitanaris, R. Stauber, G.N. Pavlakis, A. Wlodawer, The structural basis for spectral variations in green fluorescent protein, Nat. Struct. Mol. Biol. 4 (5) (1997) 361-365.

[44] M. Chattoraj, B.A. King, G.U. Bublitz, S.G. Boxer, Ultra-fast excited state dynamics in green fluorescent protein: multiple states and proton transfer, Proc. Nat. Acad. Sci. 93 (16) (1996) 8362-8367.

[45] T. Creemers, A. Lock, V. Subramaniam, T. Jovin, S. Völker, Three photoconvertible forms of green fluorescent protein identified by spectral hole-burning, Nat. Struct. Mol. Biol. 6 (6) (1999) 557.

[46] E.K. Bomati, J.E. Haley, J.P. Noel, D.D. Deheyn, Spectral and structural comparison between bright and dim green fluorescent proteins in Amphioxus, Sci. Rep. 4 (2014) 5469.

[47] G. Vicidomini, H. Ta, A. Honigmann, V. Mueller, M.P. Clausen, D. Waithe, S. Galiani, E. Sezgin, A. Diaspro, S.W. Hell, et al., STED-FLCS: an advanced tool to reveal spatiotemporal heterogeneity of molecular membrane dynamics, Nano Lett. 15 (9) (2015) 5912-5918.

[48] C. Eggeling, A. Honigmann, M. Schulze, gSTED microscopy with an OPSL: cutting edge super-resolution, Optik Photonik 7 (2) (2012) 44-46.

[49] L. Lanzanò, L. Scipioni, M. Di Bona, P. Bianchini, R. Bizzarri, F. Cardarelli, G. Vicidomini, A. Diaspro, Application of the SPLIT-FLCS method to the detection of nanoscale diffusion in 3d in live cells, Biophys. J. 110 (3) (2016) 195a.

[50] L. Lanzanò, L. Scipioni, M. Di Bona, P. Bianchini, R. Bizzarri, F. Cardarelli, A. Diaspro, G. Vicidomini, Measurement of nanoscale three-dimensional diffusion in the interior of living cells by STED-FCS, Nat. Commun. 8 (1) (2017) 65.

[51] K. Ishii, T. Tahara, Two-dimensional fluorescence lifetime correlation spectroscopy. 1. Principle, J. Phys. Chem. B 117 (39) (2013) 11414-11422.

[52] K. Ishii, T. Tahara, Two-dimensional fluorescence lifetime correlation spectroscopy. 2. Application, J. Phys. Chem. B 117 (39) (2013) 11423-11432.

[53] T. Otosu, K. Ishii, T. Tahara, Microsecond protein dynamics observed at the single-molecule level, Nat. Commun. 6 (2015).

[54] M. Lelimousin, M. Noirclerc-Savoye, C. Lazareno-Saez, B. Paetzold, S. Le Vot, R. Chazal, P. Macheboeuf, M.J. Field, D. Bourgeois, A. Royant, Intrinsic dynamics in ECFP and cerulean control fluorescence quantum yield, Biochemistry 48 (42) (2009) 10038-10046.

[55] S.T. Hess, E.D. Sheets, A. Wagenknecht-Wiesner, A.A. Heikal, Quantitative analysis of the fluorescence properties of intrinsically fluorescent proteins in living cells, Biophys. J. 85 (4) (2003) 2566-2580.

[56] J.W. Borst, M. Willemse, R. Slijkhuis, G. Van Der Krogt, S.P. Laptenok, K. Jalink, B. Wieringa, J.A. Fransen, ATP changes the fluorescence lifetime of cyan fluorescent protein via an interaction with His148, PLoS One 5 (11) (2010) e13862.

[57] A.A. Heikal, S.T. Hess, G.S. Baird, R.Y. Tsien, W.W. Webb, Molecular spectroscopy and dynamics of intrinsically fluorescent proteins: coral red (dsred) and yellow (citrine), Proc. Nat. Acad. Sci. 97 (22) (2000) 11996-12001.

[58] B. Schuler, E.A. Lipman, W.A. Eaton, Probing the free-energy surface for protein folding with single-molecule fluorescence spectroscopy, Nature 419 (6908) (2002) 743-747.

[59] E.A. Lipman, B. Schuler, O. Bakajin, W.A. Eaton, Single-molecule measurement of protein folding kinetics, Science 301 (5637) (2003) 1233-1235.

[60] B. Schuler, W.A. Eaton, Protein folding studied by single-molecule FRET, Curr. Opin. Struct. Biol. 18 (1) (2008) 16-26.

[61] S. Weiss, Measuring conformational dynamics of biomolecules by single molecule fluorescence spectroscopy, Nat. Struct. Biol. 7 (9) (2000).

[62] R. Roy, S. Hohng, T. Ha, A practical guide to single-molecule FRET, Nat. Methods 5 (6) (2008) 507-516.

[63] I. Gopich, A. Szabo, Theory of photon statistics in single-molecule Förster resonance energy transfer, J. Chem. Phys. 122 (1) (2005). 014707.

[64] I.V. Gopich, A. Szabo, Theory of the statistics of kinetic transitions with application to single-molecule enzyme catalysis, J. Chem. Phys. 124 (15) (2006) 154712

[65] S.A. McKinney, C. Joo, T. Ha, Analysis of single-molecule FRET trajectories using hidden Markov modeling, Biophys. J. 91 (5) (2006) 1941-1951. 
[66] I.V. Gopich, D. Nettels, B. Schuler, A. Szabo, Protein dynamics from singlemolecule fluorescence intensity correlation functions, J. Chem. Phys. 131 (9) (2009). 09B603.

[67] M. Pirchi, R. Tsukanov, R. Khamis, T.E. Tomov, Y. Berger, D.C. Khara, H. Volkov, G. Haran, E Nir Photon-by-photon hidden Markov model analysis for microsecond single-molecule FRET kinetics, J. Phys. Chem. B 120 (51) (2016) 13065-13075.

[68] I.V. Gopich, A. Szabo, Theory of the energy transfer efficiency and fluorescence lifetime distribution in single-molecule FRET, Proc. Nat. Acad. Sci. 109 (20) (2012) 7747-7752.

[69] A.I. Chizhik, J. Rother, I. Gregor, A. Janshoff, J. Enderlein, Metal-induced energy transfer for live cell nanoscopy, Nat. Photonics 8 (2) (2014) 124-127.
[70] N. Karedla, A.I. Chizhik, I. Gregor, A.M. Chizhik, O. Schulz, J. Enderlein, Singlemolecule metal-induced energy transfer (smMIET): resolving nanometer distances at the single-molecule level, ChemPhysChem 15 (4) (2014) 705-711.

[71] A.M. Chizhik, D. Ruhlandt, J. Pfaff, N. Karedla, A.I. Chizhik, I. Gregor, R.H. Kehlenbach, J. Enderlein, Three-dimensional reconstruction of nuclear envelope architecture using dual-color metal-induced energy transfer imaging, ACS Nano (2017).

[72] B. Hellenkamp, S. Schmid, O. Doroshenko, O. Opanasyuk, R. Kühnemuth, S.R. Adariani, A. Barth, V. Birkedal, M.E. Bowen, H. Chen, et al., Precision and accuracy of single-molecule FRET measurements - a worldwide benchmark study, arXiv preprint arXiv: 1710.03807, 2017. 


\section{Chapter 4}

\section{Metal- and Graphene-Induced Energy Transfer (MIET and GIET)}

\subsection{Manuscript: Graphene-based metal-induced en- ergy transfer for sub-nanometer optical localiza- tion}

A recent powerful extension of MIET is graphene-induced energy transfer or GIET where the metal layer is replaced by a single graphene sheet as the quenching layer. The working range of GIET is only $\sim 25 \mathrm{~nm}$, but its spatial resolution is improved almost tenfold as compared to MIET. The physical working principle of GIET is similar to MIET. Graphene is a nearly transparent semi-metal composed of a monoatomic crystalline sheet of carbon with conjugated $\pi$ electrons. Fluorescent molecules in close vicinity to such a system are efficiently quenched via resonant energy transfer from their excited states to excitations of electron-hole pairs in the graphene [172]. In my work ref. [24], we exploited this quenching property of graphene to localize single fluorophores along the optical axial. Furthermore, I used GIET to measured absolute distances of fluorophores residing in the two leaflets of supported lipid bilayers (SLBs). I could also measure the distance of the bottom leaflet from the support, which was found to be surprisingly large $(\sim 1 \mathrm{~nm})$.

The original research article published as "Arindam Ghosh, Akshita Sharma, Alexey I. Chizhik, Sebastian Isbaner, Daja Ruhlandt, Roman Tsukanov, Ingo Gregor, Narain Karedla and Jörg Enderlein. Graphene-based metal-induced energy transfer for subnanometre optical localization. Nature Photonics 13(12):860-865, 2019." is presented. 
A.G. was involved in conceiving the project, performed lifetime and defocused imaging measurements, carried out data analysis and co-wrote the manuscript.

Reproduced with permission, Copyright 2019, Springer Nature.

The published research article is currently available at https://www . nature.com/articles/ s41566-019-0510-7 


\title{
Graphene-based metal-induced energy transfer for sub-nanometre optical localization
}

\author{
Arindam Ghosh', Akshita Sharma1, Alexey I. Chizhik', Sebastian Isbaner 다', Daja Ruhlandt ${ }^{1}$, \\ Roman Tsukanov (1) ${ }^{1}$, Ingo Gregor', Narain Karedla ${ }^{1,2 \star}$ and Jörg Enderlein (1) ${ }^{1,3 \star}$
}

\begin{abstract}
Single-molecule fluorescence imaging has become an indispensable tool for almost all fields of research, from fundamental physics to the life sciences. Among its most important applications is single-molecule localization super-resolution microscopy (SMLM) (for example, photoactivated localization microscopy (PALM)', stochastic optical reconstruction microscopy (STORM) ${ }^{2}$, fluorescent PALM (fPALM) ${ }^{3}$, direct STORM (dSTORM) ${ }^{4}$ and point accumulation for imaging in nanoscale topography (PAINT) ${ }^{5}$ ), which uses the fact that the centre position of a single molecule's image can be determined with much higher accuracy than the size of that image itself. However, a big challenge of SMLM is to achieve super-resolution along the third dimension as well. Recently, metal-induced energy transfer (MIET) was introduced to axially localize fluorescent emitters ${ }^{6-9}$. This exploits the energy transfer from an excited fluorophore to plasmons in a thin metal film. Here, we show that by using graphene as the 'metal' layer, one can increase the localization accuracy of MIET by nearly tenfold. We demonstrate this by axially localizing single emitters and by measuring the thickness of lipid bilayers with ångström accuracy.
\end{abstract}

For the localization of a single molecule not only laterally, but also axially, various techniques have been invented, including astigmatic imaging $^{10}$, bi-plane imaging ${ }^{11}$ and wavefront shaping ${ }^{12}$. However, all these methods yield an axial localization accuracy that is typically three to five times worse than the lateral localization accuracy The only exceptions are interference-based methods ${ }^{13,14}$, but this is at the cost of elevated technical complexity. However, it should be mentioned that recently developed methods such as direct optical nanoscopy with axially localized detection (DONALD) ${ }^{15}$ or self-interference (SELFI) microscopy ${ }^{16}$ allow one to achieve a spatial resolution on the order of several tens of nanometres along all three dimensions. As mentioned above, metal-induced energy transfer (MIET) uses the fluorescence modulation caused by the electromagnetic coupling of an emitter's excited state to surface plasmons in a metal layer to calculate the emitter's position in relation to the metal surface. Similar to Förster resonance energy transfer (FRET), this coupling shows a strong distance dependence, but over a much wider range (up to $150 \mathrm{~nm}$ ) than FRET, which enables high-precision axial localization of fluorescent emitters over a similar axial range as covered in totalinternal reflection fluorescence (TIRF) microscopy. If $L$ denotes the range of this interaction, then the achievable localization accuracy scales with the number $N$ of detected photons as $L / \sqrt{N}$. This is similar to the lateral localization accuracy in SMLM, where $L$ would be the size of the point spread function. For metal films of gold or silver,
$L$ ranges from $\sim 150 \mathrm{~nm}$ to $200 \mathrm{~nm}$, which allows us to localize single molecules to $\sim 5 \mathrm{~nm}$ to $6 \mathrm{~nm}$ at a photon budget of 1,000 photons $^{6-9}$.

This situation changes significantly when replacing the metal with graphene. Graphene is a two-dimensional (2D) material composed of a monoatomic crystalline sheet of carbon with conjugated $\pi$ electrons. Its electronic energy spectrum corresponds to a zerogap semiconductor ${ }^{17,18}$. The peculiar optical properties of graphene monolayers have been thoroughly characterized and described ${ }^{19}$ Similar to metal-based MIET, a fluorescent emitter can efficiently couple to graphene via a distance-dependent electromagnetic nearfield coupling. However, this coupling is very similar to classical FRET, but here the acceptor is replaced by a planar layer of graphene (which plays the role of a 'dark quencher'). Similar to FRET, the coupling range is smaller by approximately one order of magnitude than that for MIET with a metal (Fig. 1b). This leads to a tenfold better axial resolution and thus provides sub-nanometre localization accuracy at photon budgets as low as 1,000 photons.

Next, we give a brief theoretical description of graphene-based MIET (gMIET - we will keep the acronym MIET also for graphene) which will also be needed for data evaluation, and then present experimental results for the axial localization of immobilized molecules and for distance measurements across a fluorescently labelled supported lipid bilayer.

The geometry of a gMIET experiment is shown in Fig. 1a. A fluorescent molecule is located at a distance $z_{0}$ above a substrate that consists of a single sheet of graphene sandwiched between a glass cover slide and a thin silicon dioxide spacer layer. Fluorescence excitation and detection are performed through that substrate, from below. The electrodynamic coupling of the excited state of the molecule to graphene modulates its transition from the excited state to the ground state. We model this interaction by treating the excited molecule as an ideal electric dipole emitter and the graphene as a layer of matter with specific thickness and (complex-valued) bulk refractive index. Solving Maxwell's equations for such a system then leads to an expression for the emission power, $S\left(\theta, z_{0}\right)$, of the electric dipole emitter as a function of dipole distance $z_{0}$ and orientation (described by the angle $\theta$ between the dipole axis and the vertical axis $)^{20-22}$. The emission power $S\left(\theta, z_{0}\right)$ itself is inversely proportional to the radiative transition rate of the molecule's excited state to its ground state, and together with the non-radiative rate due, for example, to collisions with surrounding molecules, defines the measurable excited-state (fluorescence) lifetime $\tau_{\dot{f}}$.

$$
\frac{\tau_{\mathrm{f}}\left(\theta, z_{0}\right)}{\tau_{0}}=\frac{S_{0}}{\phi S\left(\theta, z_{0}\right)+(1-\phi) S_{0}}
$$

'Third Institute of Physics - Biophysics, Georg August University, Göttingen, Germany. ${ }^{2}$ Physical and Theoretical Chemistry, University of Oxford, Oxford, UK. ${ }^{3}$ Cluster of Excellence 'Multiscale Bioimaging: from Molecular Machines to Networks of Excitable Cells' (MBExC), Georg August University, Göttingen, Germany. *e-mail: narain.karedla@chem.ox.ac.uk; jenderl@gwdg.de 


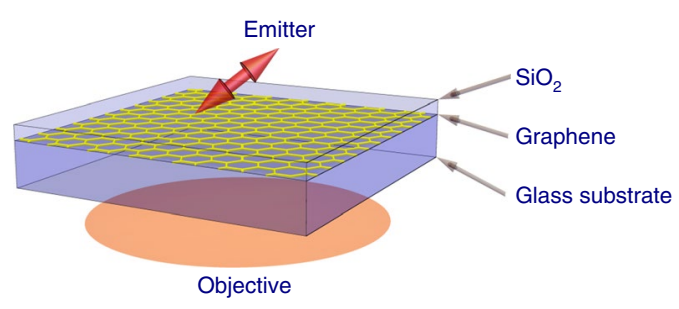

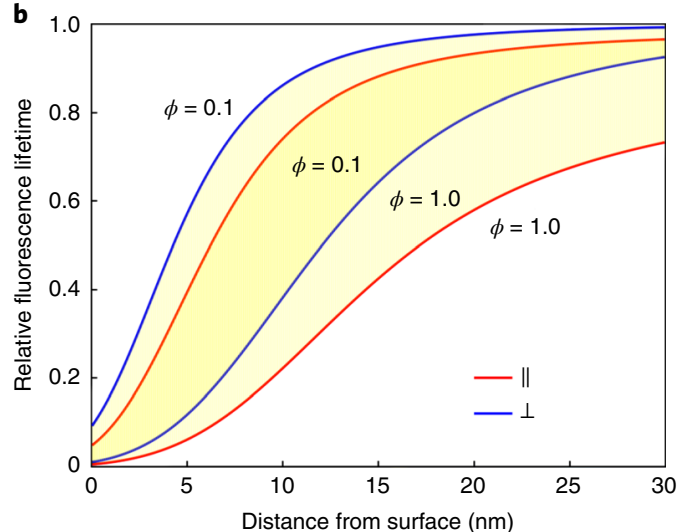

Fig. 1 | Graphene-based MIET. a, Schematic of a gMIET experiment. b, Relative fluorescence lifetime $\tau / \tau_{0}$ as a function of distance $z_{0}$ of a molecule from the surface of a silica spacer of $5 \mathrm{~nm}$ thickness. The blue and red curves refer to molecules oriented perpendicular and parallel to the surface, respectively, and the shaded region covers quantum yield values between $\phi=0.1$ and $\phi=1.0$. Calculations were performed for a single emission wavelength of $680 \mathrm{~nm}$. The refractive index of the upper medium was set to 1 and that of glass to 1.52 .

Here, $\phi$ is the quantum yield (QY), $\tau_{0}$ is the free-space lifetime in the absence of MIET, $S_{0}$ is the free-space emission power of an ideal electric dipole emitter, $S_{0}=c n k_{0}^{4} p^{2} / 3$, with $c$ being the speed of light, $k_{0}$ the wavevector in vacuum, $n$ the refractive index of water and $p$ the amplitude of the dipole moment vector. The radiative emission rate $S\left(\theta, z_{0}\right)$ itself can be decomposed as

$$
S\left(\theta, z_{0}\right)=S_{\perp}\left(z_{0}\right) \cos ^{2} \theta+S_{\|}\left(z_{0}\right) \sin ^{2} \theta
$$

where $S_{\perp}\left(z_{0}\right)$ and $S_{\|}\left(z_{0}\right)$ are the radiative emission rates of emitters oriented perfectly perpendicular and parallel to the substrate, respectively.

Figure $1 \mathrm{~b}$ shows the calculated relative lifetime $\left(\tau_{\mathrm{f}} / \tau_{0}\right)$ as a function of distance $z_{0}$ for the limiting cases of emitters oriented either perfectly parallel or orthogonal to the surface, and for quantum yield values between 0.1 and 1.0. For these and all subsequent calculations, we adopted a graphene layer thickness of $0.34 \mathrm{~nm}$ and a refractive index of $n_{\text {graphene }}=2.77+1.41 i$ (corresponding to an emission wavelength of $680 \mathrm{~nm})^{19}$. The thickness of the silica layer (refractive index $n_{\mathrm{SiO}_{2}}=1.46$ ) above the graphene was set to $5 \mathrm{~nm}$ and the half space above is water (refractive index $n_{\mathrm{H}_{2} \mathrm{O}}=1.33$ ).

As can be seen, graphene-induced fluorescence quenching/ modulation takes place within the first $\sim 25 \mathrm{~nm}$, on a much shorter length scale than the typical interaction range of $\sim 150 \mathrm{~nm}$ for gold or silver layers. The core idea of MIET is to use this strong lifetimeon-distance dependence for converting a measured lifetime into a distance value by using the model curve as shown in Fig. 1b. This requires knowledge of the intrinsic QY of the used dye, which has to be measured a priori, for example with a nanocavity resonator $\operatorname{method}^{23}$. To calculate gMIET curves for the data evaluation of experimental measurements, one has also to take into account that real dyes exhibit broad emission spectra. In that case, one has to calculate $S\left(\theta, z_{0}\right)$ also as a function of wavelength using the known dispersion relation for graphene ${ }^{19}$ and to weigh the final result with the emission spectrum of the dye (see Supplementary Section 'Conversion of lifetime values to distance values').

As a proof of principle, we checked gMIET by measuring the absolute distances of single molecules from a surface for samples with a well-known sample geometry. We spin-coated fluorescent dye molecules (Atto 655) on top of three different substrates with silica spacer thickness values of $10 \mathrm{~nm}, 15 \mathrm{~nm}$ and $20 \mathrm{~nm}$, defining the distance of the molecules from the graphene layer (see Supplementary Sections 'Substrate preparation' and 'Sample preparation'). Fluorescence intensity and lifetime images of the samples were taken with a confocal laser scanning microscope, using pulsed laser excitation and time-correlated single-photon counting (TCSPC) for fluorescence lifetime measurements (laser focus diameter of $\sim 220 \mathrm{~nm}$, total excitation power of $20 \mu \mathrm{W}$, laser pulse width and repetition rate of $50 \mathrm{ps}$ and $40 \mathrm{MHz}$, respectively, scan speed of $1 \mu \mathrm{m} / 40 \mathrm{~ms}$ ) (see Supplementary Section 'Fluorescence lifetime measurements'). In each scan image (Supplementary Fig. 1), individual molecules were identified and their corresponding photons pooled to obtain single-molecule fluorescence decay curves. The average numbers of detected photons from one molecule were $1,489,4,786$ and 6,033 , respectively, showing the increasing fluorescence quenching by graphene with decreasing distance.

The single-molecule fluorescence decay curves were tail-fitted with a single-exponential decay function (discarding all photons that where detected faster than half a nanosecond after the laser excitation pulse), thus yielding a fluorescence decay time for each identified molecule (see Supplementary Section 'Lifetime fitting' and Supplementary Fig. 2). Typical decay curves and fits for single molecules are shown in Fig. 2a. Histograms of all determined lifetime values for all three samples are shown in Fig. 2b. In the theoretical discussion above, when calculating the lifetime-versus-distance curves, we assumed that the emitters are rapidly rotating on a much faster timescale than the fluorescence decay time, which is the typical case for most samples where dye molecules are attached to structures of interest (proteins, DNA and so on) via short highly flexible carbon linkers. For the current sample of immobilized molecules, this assumption is no longer correct. To determine the orientation distribution of the immobilized molecules, we used defocused imaging $^{24,25}$. In Fig. 2c, we present defocused images of the molecules on the graphene/silica substrate (see Supplementary Section 'Defocused imaging'), from which we can extract the distribution $\rho(\theta)$ of emission dipole inclination angles $\theta$ with respect to the optical axis as shown in Fig. 2d. Knowing this distribution, equation (1) can be used to find an orientation-averaged gMIET curve as

$$
\left\langle\tau_{\mathrm{f}}\left(z_{0}\right)\right\rangle=\int_{0}^{\pi / 2} \mathrm{~d} \theta \sin \theta \rho(\theta) \tau_{\mathrm{f}}\left(z_{0}, \theta\right)
$$

To calculate this curve, we have to know the quantum yield and the free-space lifetime for the immobilized molecules, which we measured using our recently developed nanocavity method ${ }^{23}$ and employing the known orientation distribution from Fig. $2 \mathrm{~d}$. The determined values are $\tau_{0}=2.9 \mathrm{~ns}$ for the free-space lifetime and $\phi=0.2$ for the quantum yield. The resulting gMIET curve is shown 

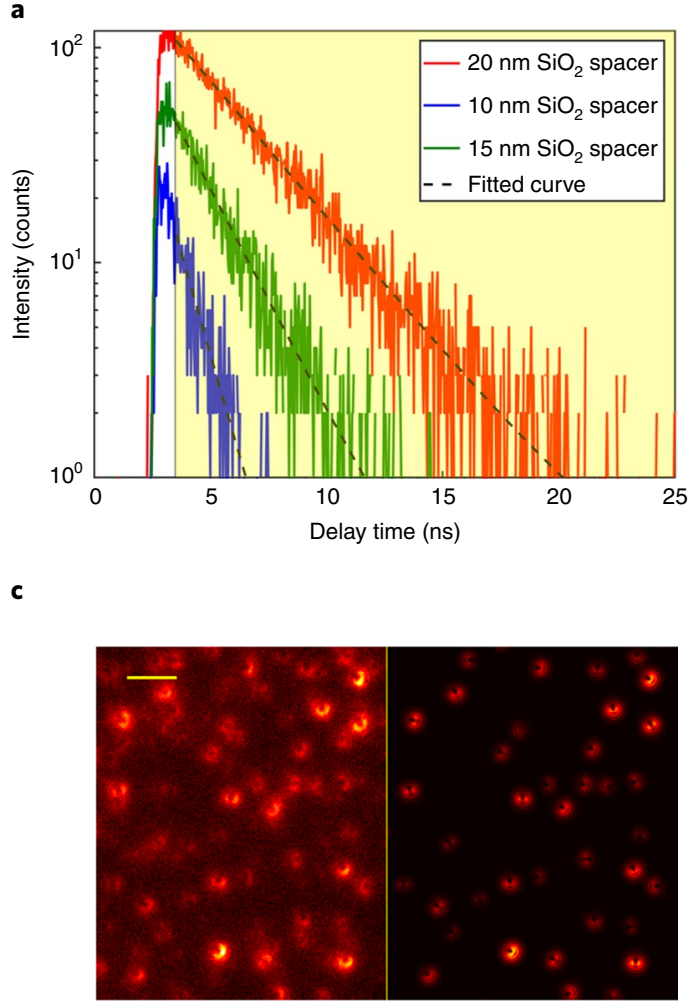

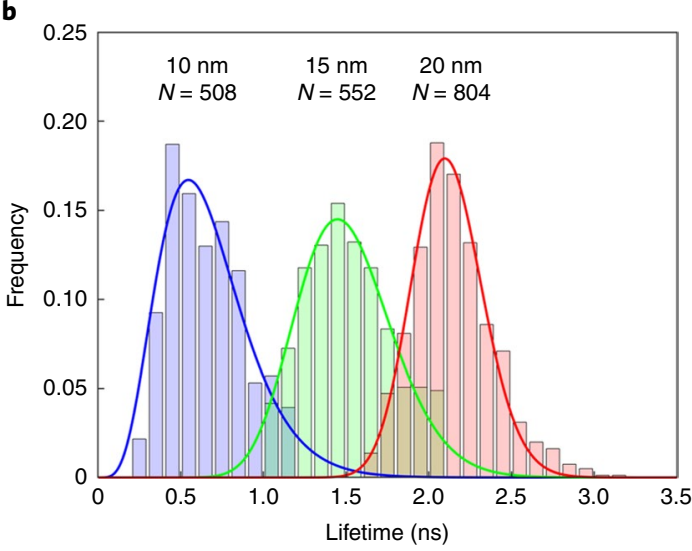

d

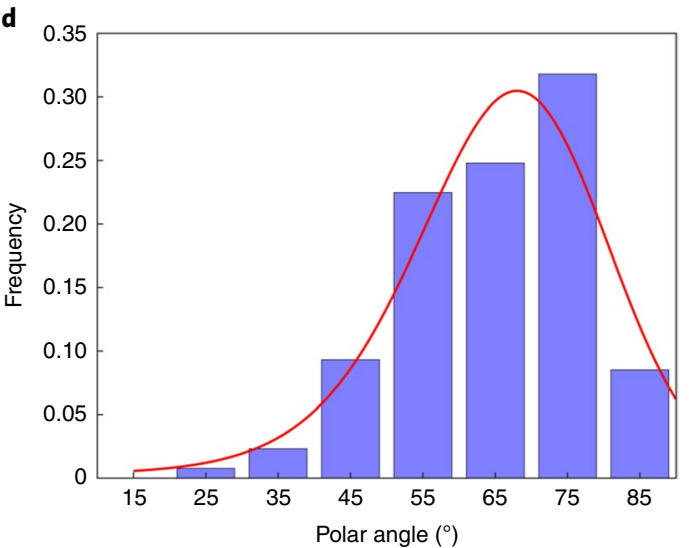

e

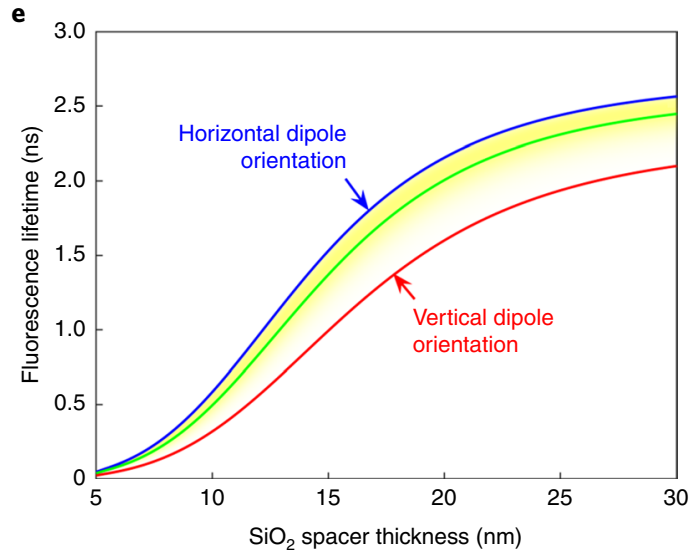

f

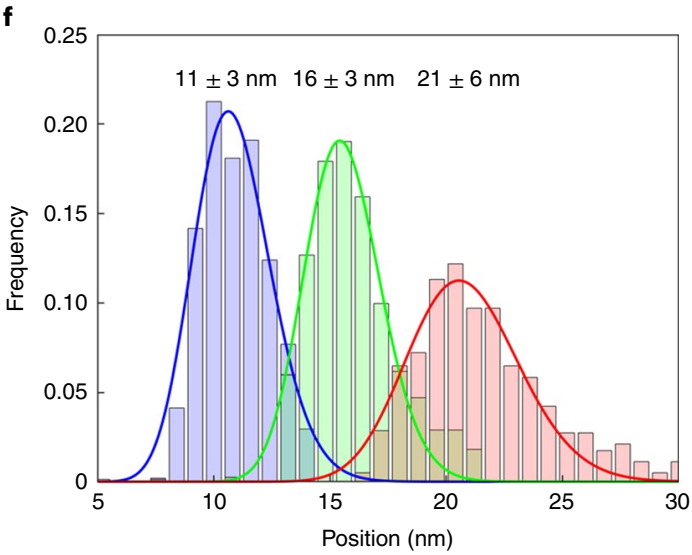

Fig. 2 | Axial localization of single molecules with graphene-based MIET. a, Example fluorescence decay curves for three different molecules on the three studied substrates with a $10 \mathrm{~nm}, 15 \mathrm{~nm}$ and $20 \mathrm{~nm} \mathrm{SiO}$ spacer on top of the graphene layer. The yellow shaded region is used for a maximum likelihood mono-exponential tail fit of the fluorescence decay time. $\mathbf{b}$, Lifetime distributions as obtained by tail-fitting the decay curves of individual molecules. Numbers above the distributions indicate how many molecules contributed to the corresponding distribution. The mean numbers of detected photons per molecule that were used for tail-fitting the lifetime were 1,489, 4,786 and 6,033 for the $10 \mathrm{~nm}, 15 \mathrm{~nm}$ and $20 \mathrm{~nm}$ samples, respectively. Solid lines represent fits of a general gamma distribution to the histograms. c, Measured defocused wide-field image of single molecules (left) of Atto 655 deposited on a glass $/ \mathrm{SiO}_{2}$ sample and theoretically fitted defocused image (right). Scale bar, $1 \mu \mathrm{m}$. d, Bar histogram of molecular orientation distribution (angle towards the vertical axes) as extracted from the defocused images shown in c. e, Calculated gMIET calibration curves: blue and red curves show the gMIET calibration curves that one would have for purely horizontally or vertically oriented molecules. The yellow density plot shows a distribution of gMIET curves where the shading reflects the weight proportional to the orientation distribution from $\mathbf{d}$. The green curve is the corresponding orientationaveraged gMIET calibration curve as used for subsequently converting the experimental lifetime to distance values. $\mathbf{f}$, Distance distributions as obtained by converting the lifetimes from $\mathbf{b}$ into distance values by using the green gMIET calibration curve from $\mathbf{e}$.

in Fig. 2e together with the curves for both horizontal and vertical dipole orientation. In this figure, the yellow shading represents a superposition of gMIET curves for all possible orientation angles $\theta$ where the shading intensity corresponds to the value of $\rho(\theta)$. Using the orientation-averaged gMIET curve, we converted the measured lifetime of each molecule into a distance value. The resulting histograms are shown in Fig. 2f, where above each distribution its mean distance value is indicated (see Supplementary Section 'Conversion 

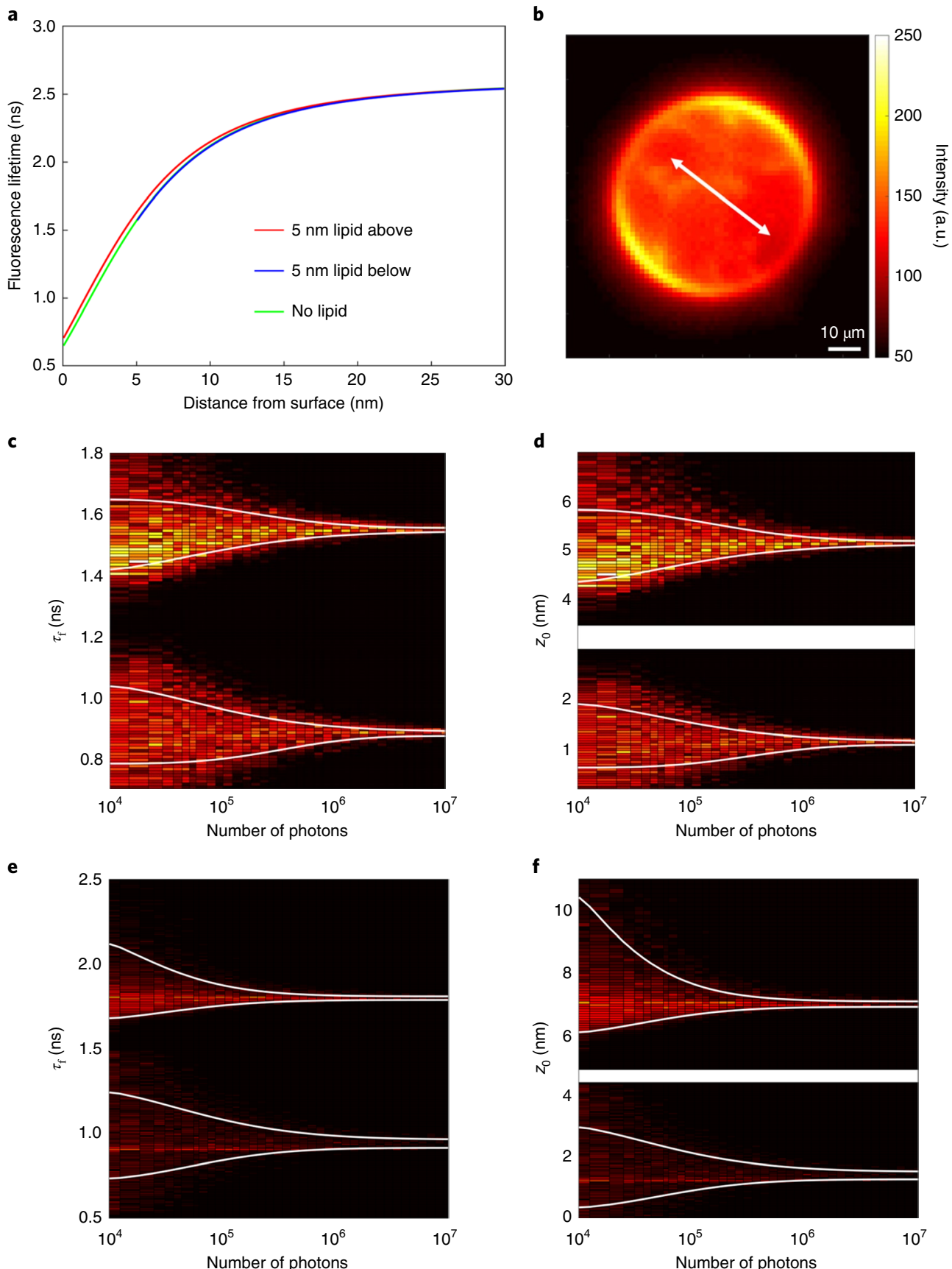

Fig. 3 | Graphene-based MIET measurement of the thickness of SLBs. a, MIET curves for the bilayer experiment. To estimate the impact of an $\sim 5$-nmthick bilayer of refractive index 1.46, we calculated MIET curves assuming no bilayer was present, with a bilayer above and with a bilayer below the fluorophore's position. b, Image of a GUV under linearly polarized excitation (the double-headed arrow indicates the excitation polarization). c, Lifetime distributions for DLPC as a function of photons per TCSPC curve. The solid white lines show the mean value \pm the square root of the variance of the lifetime distributions as a function of photon number. d, Distance distribution corresponding to $\mathbf{c}$. For the conversion from lifetime to distance, we used the blue curve from a for all lifetime values larger than $1.25 \mathrm{~ns}$ and the red curve for all lifetime values smaller than $1.25 \mathrm{~ns}$. The white gap reflects the difference between the red and blue curve in a. e,f, Distributions similar to those in $\mathbf{c}, \mathbf{d}$ but for DOPC. To convert the lifetime values of $\mathbf{e}$ into the distance values in $\mathbf{f}$, we used the blue curve from a for all lifetime values larger than $1.5 \mathrm{~ns}$ and the red curve for all lifetime values smaller than $1.5 \mathrm{~ns}$.

of lifetime values to distance values'). As can be seen, there exists a small systematic bias of $\sim 1 \mathrm{~nm}$ towards larger distance values than those expected from the sample preparation. We identify two possible origins of this deviation. One origin can be the surface roughness of the samples. We performed atomic force microscopy measurements of the silica layer surface of our samples and found a rather large root-mean-square value for the surface roughness ranging from $1.5 \mathrm{~nm}$ to $2.1 \mathrm{~nm}$ (Supplementary Fig. 5). This may also explain why we observe such a broad dipole orientation distribution, in contrast to similar experiments for molecules deposited on flatter substrates ${ }^{8}$. Another origin of the observed deviation may be the surface-induced heterogeneity of the photophysical parameters of the immobilized molecules. Previous experiments have shown that surface-immobilized molecules can exhibit a rather broad distribution of quantum yield values ${ }^{26}$. In our case, this leads to a broadening of the distance value distributions in Fig. 2f, but may 
also introduce a slight systematic bias of the average distance values with respect to the actual ones. However, it should be emphasized that the relative differences of vertical positions are reproduced correctly, showing a $5 \mathrm{~nm}$ increase from sample to sample. As another validation of gMIET, we performed axial localizations of two emitters on top of a DNA origami platform. Using a custom-designed DNA origami, we positioned Atto 647N fluorophores at two specific heights and used gMIET to localize them axially with nanometre accuracy (see Supplementary Section 'Multi-emitter localization on top of DNA origami using gMIET' and Supplementary Fig. 3). To demonstrate that gMIET works across the full visible spectrum, we also performed gMIET measurements for the blue-green dye Atto 488. As in the case of Atto 655, we sparsely deposited single Atto 488 molecules on graphene samples coated with an $\mathrm{SiO}_{2}$ spacer of $10 \mathrm{~nm}$ thickness and determined their distance from the graphene layer (see Supplementary Section 'Axial localization of single Atto 488 molecules using gMIET' and Supplementary Fig. 4).

An important application of gMIET could be the study of lipid bilayers and cellular membranes, which have typical thicknesses of $\sim 5 \mathrm{~nm}$ that cannot easily be resolved by current fluorescence imaging methods. Here, we applied gMIET to estimate the thickness of supported lipid bilayers (SLBs) by localizing fluorescent dyes attached to lipid head groups in the bottom and top leaflets of the SLB. Using vesicle fusion, we prepared pure dioleoylphosphatidylcholine (DOPC) and dilauroylphosphatidylcholine (DLPC) SLBs on our graphene substrates with a low percentage of lipids that were fluorescently labelled with the dye Atto 655 (see Supplementary Section 'Sample preparation' for the SLB preparation protocol). We recorded fluorescence decay curves from these samples at $20^{\circ} \mathrm{C}$ by scanning an area of $5 \mu \mathrm{m} \times 5 \mu \mathrm{m}$, and we fitted these decay curves with a bi-exponential function. Distributions of these fitted fluorescence decay times as a function of the number $N$ of photons per TCSPC curve are shown in Fig. $3 c$ for DOPC and in Fig. $3 e$ for DLPC (the total number of recorded photons was $4.3 \times 10^{7}$ for the DOPC sample and $1.6 \times 10^{8}$ for the DLPC sample). To generate these distributions, we chopped the full measurement into bunches of $N$ photons and fitted the corresponding TCSPC curves.

As discussed above, to convert these lifetime values into correct distance values, additional information on dye orientation is required. For this purpose, we prepared giant unilamellar vesicles (GUVs) using the same lipids and labelling, and imaged them under polarized excitation light (see Supplementary Section 'Dye orientation in GUVs' $)^{27}$. The observed intensity distribution shown in Fig. $3 \mathrm{~b}$ is consistent with a dye orientation parallel to the bilayer surface Furthermore, we again used the nanocavity method to measure the free-space lifetime and QY of the lipid-bound Atto $655^{28}$. For the DOPC-bound Atto 655, we found values of $\tau_{0}=2.6 \mathrm{~ns}$ for the freespace lifetime and $\phi=0.36$ for the QY, and we used these values also for the DLPC SLBs. It should be noted that coupling of fluorophores to a lipid changes its local environment, and this does impact its non-radiative decay rate and thus QY. As a result, QY values for molecules in aqueous solution and attached to a lipid can be quite different. In the model calculation, we took into account the presence of the bilayer, assuming a bilayer refractive index of 1.46 and a rough estimate of the bilayer thickness of $5 \mathrm{~nm}$.

Using the resulting gMIET calibration curve as shown in Fig. 3a, we converted the lifetime values into distance values, as shown in Fig. $3 \mathrm{~d}$ for the DLPC SLB and in Fig. $3 \mathrm{f}$ for the DOPC SLB. For this conversion, we used the gMIET curve labelled as ' $5 \mathrm{~nm}$ lipid above' in Fig. 3a for the short lifetime component (bottom leaflet) and the curve labelled as ' $5 \mathrm{~nm}$ lipid below' for the long lifetime component (top leaflet). As can be seen from Fig. 3d,f, the relative width of the distribution becomes very small for a large number of photon counts per TCSPC measurement. In the limit of very large numbers (right edge of figures at $10^{7}$ photons), we determined the following values for dye positions: for the bottom and top leaflets of DLPC we find
$1.09 \pm 0.04 \mathrm{~nm}$ and $5.13 \pm 0.04 \mathrm{~nm}$, respectively, and for the bottom and top leaflets of DOPC we find $1.4 \pm 0.1 \mathrm{~nm}$ and $6.9 \pm 0.1 \mathrm{~nm}$. Thus, the distances between top and bottom leaflets are $4.04 \pm 0.06 \mathrm{~nm}$ for the DLPC SLB and $5.5 \pm 0.2 \mathrm{~nm}$ for the DOPC SLB. These values are in excellent agreement with literature values ${ }^{29,30}$, which report head-group-to-head-group distances of $3.3 \pm 0.07 \mathrm{~nm}$ for DLPC and $4.6 \pm 0.2 \mathrm{~nm}$ for DOPC. In our measurements, the dye distance values are larger due to the finite linker length and the size of the dyes themselves. Amazingly, for DOPC the reported thickness value for the hydration layer between substrate and bottom leaflet of $1.79 \mathrm{~nm}$ (ref. ${ }^{31}$ ) also agrees surprisingly well with our finding of $1.4 \pm 0.1 \mathrm{~nm}$ for the bottom-leaflet-dye-to-substrate distance.

In summary, we have demonstrated that gMIET, with its steep lifetime-on-distance dependence, allows us to enhance the axial resolution of MIET from a few nanometres to the ångström level. The excellent optical transparency of graphene compared to gold films makes gMIET an extremely sensitive tool for single-molecule studies where the photon budget is limited. Moreover, any fluorescent dye can be exploited for gMIET as long as its fluorescence decay behaviour and QY are well known a priori. We believe that the technical simplicity of MIET that has led to many applications in the life sciences ${ }^{6,32-35}$, along with the ample availability of commercial graphene substrates, will make gMIET a powerful tool for numerous applications where one is interested in resolving distances with sub-nanometre accuracy, such as in artificial bilayers, cellular membranes or macromolecular complexes. An attractive extension of gMIET will be its combination with the recently introduced MINFLUX ${ }^{36}$, which would add similarly high lateral resolution at comparable photon budgets, thus realizing 3D localization with sub-nanometre isotropic resolution ${ }^{9}$.

\section{Online content}

Any methods, additional references, Nature Research reporting summaries, source data, statements of code and data availability and associated accession codes are available at https://doi.org/10.1038/ s41566-019-0510-7.

\section{Data availability}

The data that support the plots within this paper and other findings of this study are available from the corresponding authors upon reasonable request.

\section{Code availability}

All Matlab routines and codes used for data analysis of this study are available from the corresponding authors upon request.

Received: 8 March 2019; Accepted: 16 July 2019;

Published online: 2 September 2019

\section{References}

1. Betzig, E. et al. Imaging intracellular fluorescent proteins at nanometer resolution. Science 313, 1642-1645 (2006)

2. Rust, M. J., Bates, M. \& Zhuang, X. Sub-diffraction-limit imaging by stochastic optical reconstruction microscopy (STORM). Nat. Methods 3, 793-796 (2006).

3. Hess, S. T., Girirajan, T. P. \& Mason, M. D. Ultra-high resolution imaging by fluorescence photoactivation localization microscopy. Biophys. J. 91, 4258-4272 (2006).

4. Heilemann, M. et al. Subdiffraction-resolution fluorescence imaging with conventional fluorescent probes. Angew. Chem. Int. Ed. 47, 6172-6176 (2008).

5. Sharonov, A. \& Hochstrasser, R. M. Wide-field subdiffraction imaging by accumulated binding of diffusing probes. Proc. Natl Acad. Sci. USA 103, 18911-18916 (2006)

6. Chizhik, A. I., Rother, J., Gregor, I., Janshoff, A. \& Enderlein, J. Metal-induced energy transfer for live cell nanoscopy. Nat. Photon. 8, 124-127 (2014).

7. Isbaner, S. et al. Axial colocalization of single molecules with nanometer accuracy using metal-induced energy transfer. Nano Lett. 18, 2616-2622 (2018).

8. Karedla, N. et al. Single-molecule metal-induced energy transfer (smMIET): resolving nanometer distances at the single-molecule level. ChemPhysChem 15, 705-711 (2014). 
9. Karedla, N. et al. Three-dimensional single-molecule localization with nanometer accuracy using metal-induced energy transfer (MIET) imaging. J. Chem. Phys. 148, 204201 (2018).

10. Huang, B., Wang, W., Bates, M. \& Zhuang, X. Three-dimensional superresolution imaging by stochastic optical reconstruction microscopy. Science 319, 810-813 (2008)

11. Juette, M. F. et al. Three-dimensional sub- $100 \mathrm{~nm}$ resolution fluorescence microscopy of thick samples. Nat. Methods 5, 527-529 (2008).

12. Backlund, M. P. et al. Simultaneous, accurate measurement of the $3 \mathrm{D}$ position and orientation of single molecules. Proc. Natl Acad. Sci. USA 109, 19087-19092 (2012).

13. Shtengel, G. et al. Interferometric fluorescent super-resolution microscopy resolves 3D cellular ultrastructure. Proc. Natl Acad. Sci. USA 106, 3125-3130 (2009).

14. Aquino, D. et al. Two-color nanoscopy of three-dimensional volumes by $4 \mathrm{Pi}$ detection of stochastically switched fluorophores. Nat. Methods $\mathbf{8}$, 353-359 (2011).

15. Bourg, N. et al. Direct optical nanoscopy with axially localized detection. Nat. Photon. 9, 587-593 (2015).

16. Bon, P. et al. Self-interference 3D super-resolution microscopy for deep tissue investigations. Nat. Methods 15, 449-454 (2018).

17. Novoselov, K. S. et al. Electric field effect in atomically thin carbon films. Science 306, 666-669 (2004)

18. Wallace, P. R. The band theory of graphite. Phys. Rev. 71, 622 (1947)

19. Weber, J., Calado, V. \& Van De Sanden, M. Optical constants of graphene measured by spectroscopic ellipsometry. Appl. Phys. Lett. 97, 091904 (2010)

20. Chance, R., Prock, A. \& Silbey, R. Molecular fluorescence and energy transfer near interfaces. Adv. Chem Phys. 37, 1-65 (1978).

21. Enderlein, J. Single-molecule fluorescence near a metal layer. Chem. Phys. 247, 1-9 (1999).

22. Chizhik, A. I. et al. Electrodynamic coupling of electric dipole emitters to a fluctuating mode density within a nanocavity. Phys. Rev. Lett. 108, 163002 (2012)

23. Chizhik, A. I., Gregor, I., Ernst, B. \& Enderlein, J. Nanocavity-based determination of absolute values of photoluminescence quantum yields ChemPhysChem 14, 505-513 (2013).

24. Böhmer, M. \& Enderlein, J. Orientation imaging of single molecules by wide-field epifluorescence microscopy. J. Opt. Soc. Am. B 20, 554-559 (2003).

25. Patra, D., Gregor, I. \& Enderlein, J. Image analysis of defocused singlemolecule images for three-dimensional molecule orientation studies. J. Phys. Chem. A 108, 6836 (2004).

26. Chizhik, A. I. et al. Probing the radiative transition of single molecules with tunable microresonator. Nano Lett. 11, 1700-1703 (2011).

27. Bagatolli, L. A. To see or not to see: lateral organization of biological membranes and fluorescence microscopy. Biochim. Biophys. Acta 1758 1541-1556 (2006).

28. Schneider, F., Ruhlandt, D., Gregor, I., Enderlein, J. \& Chizhik, A. I. Quantum yield measurements of fluorophores in lipid bilayers using a plasmonic nanocavity. J. Phys. Chem. Lett. 8, 1472-1475 (2017).

29. Kučerka, N., Nieh, M.-P. \& Katsaras, J. Fluid phase lipid areas and bilayer thicknesses of commonly used phosphatidylcholines as a function of temperature. Biochim. Biophys. Acta 1808, 2761-2771 (2011).
30. Attwood, S. J., Choi, Y. \& Leonenko, Z. Preparation of DOPC and DPPC supported planar lipid bilayers for atomic force microscopy and atomic force spectroscopy. Int. J. Mol. Sci. 14, 3514-3539 (2013).

31. Tristram-Nagle, S., Petrache, H. I. \& Nagle, J. F. Structure and interactions of fully hydrated dioleoylphosphatidylcholine bilayers. Biophys. J. 75, 917-925 (1998)

32. Chizhik, A. M. et al. Three-dimensional reconstruction of nuclear envelope architecture using dual-color metal-induced energy transfer imaging. ACS Nano 11, 11839-11846 (2017)

33. Baronsky, T. et al. Cell-substrate dynamics of the epithelial-to-mesenchymal transition. Nano Lett. 17, 3320-3326 (2017).

34. Simoncelli, S., Makarova, M., Wardley, W. \& Owen, D. M. Toward an axial nanoscale ruler for fluorescence microscopy. ACS Nano 11, 11762-11767 (2017).

35. Chizhik, A. M. et al. Dual-color metal-induced and Förster resonance energy transfer for cell nanoscopy. Mol. Biol. Cell 29, 846-851 (2018).

36. Balzarotti, F. et al. Nanometer resolution imaging and tracking of fluorescent molecules with minimal photon fluxes. Science 355, 606-612 (2016).

\section{Acknowledgements}

We are grateful to the Deutsche Forschungsgemeinschaft (DFG, German Research Foundation) for financial support through projects A06 of SFB 803, A06 of SFB 860, A05 of SFB 937 and through Germany's Excellence Strategy EXC 2067/1-390729940. We thank the Leibniz Association for financial support through project K76/2017. We also thank B. R. Brueckner for AFM measurements.

\section{Author contributions}

A.G. and N.K. co-designed the project. A.G., A.S. and I.G. performed all the lifetime and defocused imaging measurements. A.G. generated Figs. 2a,c and $3 \mathrm{~b}$ in the main text. N.K. performed analysis of the defocused imaging data and lifetime data from SLB. A.I.C. carried out the quantum yield measurements with the nanocavity and D.R. performed the corresponding data analysis. S.I. conducted lifetime fitting of singlemolecule data and wrote the corresponding section in the Supplementary Information. D.R. helped with writing the MIET routines in Matlab. R.T. designed the DNA origami sample. A.G., N.K. and J.E. carried out the final data analysis, generated all figures (except Figs. 2a,c and 3b) in the main text and wrote the main manuscript. All coworkers were involved with improving the manuscript and writing the Supplementary Information.

\section{Competing interests}

The authors declare no competing interests.

\section{Additional information}

Supplementary information is available for this paper at https://doi.org/10.1038/ s41566-019-0510-7.

Reprints and permissions information is available at www.nature.com/reprints. Correspondence and requests for materials should be addressed to N.K. or J.E. Publisher's note: Springer Nature remains neutral with regard to jurisdictional claims in published maps and institutional affiliations.

(C) The Author(s), under exclusive licence to Springer Nature Limited 2019 
In the format provided by the authors and unedited.

\title{
Graphene-based metal-induced energy transfer for sub-nanometre optical localization
}

\author{
Arindam Ghosh', Akshita Sharma', Alexey I. Chizhik', Sebastian Isbaner (1)', Daja Ruhlandt (1)',
} Roman Tsukanov ${ }^{\circledR}{ }^{1}$, Ingo Gregor ${ }^{1}$, Narain Karedla ${ }^{1,2 \star}$ and Jörg Enderlein ${ }^{1,3 \star}$

'Third Institute of Physics - Biophysics, Georg August University, Göttingen, Germany. ${ }^{2}$ Physical and Theoretical Chemistry, University of Oxford, Oxford, UK. ${ }^{3}$ Cluster of Excellence 'Multiscale Bioimaging: from Molecular Machines to Networks of Excitable Cells' (MBExC), Georg August University, Göttingen, Germany. *e-mail: narain.karedla@chem.ox.ac.uk; jenderl@gwdg.de 


\title{
Graphene-based metal-induced energy transfer for sub- nanometre optical localization
}

\author{
Arindam Ghosh ${ }^{1}$, Akshita Sharma ${ }^{1}$, Alexey I. Chizhik ${ }^{1}$, Sebastian Isbaner ${ }^{1}$, Daja Ruhlandt ${ }^{1}$, Roman \\ Tsukanov $^{1}$, Ingo Gregor ${ }^{1}$, Narain Karedla ${ }^{1,2 *} \&$ Jörg Enderlein ${ }^{1,3 *}$ \\ ${ }^{1}$ III. Institute of Physics - Biophysics, Georg August University, Friedrich-Hund-Platz 1, \\ 37077 Göttingen, Germany. \\ ${ }^{2}$ Physical and Theoretical Chemistry, University of Oxford, 12 Mansfield Road, Oxford OX1 3TA, \\ $U K$.
}

\section{Substrate preparation}

Plasma cleaned glass coverslips $(24 \mathrm{~mm} \times 24 \mathrm{~mm}$, thickness $170 \mu \mathrm{m})$ coated with a graphene monolayer (0.34 nm thickness) were purchased from Graphene Supermarket, New York, USA. These coverslips were coated in-house with $\mathrm{SiO}_{2}$ spacers of $10 \mathrm{~nm}, 15 \mathrm{~nm}$, and $20 \mathrm{~nm}$ thickness by evaporation using an electron beam source (Univex 350, Leybold) under high vacuum conditions $\left(10 \times 10^{-6} \mathrm{mbar}\right)$. Slowest rate of deposition was maintained $\left(1 \AA^{-1} \mathrm{~s}^{-1}\right)$ to ensure maximal homogeneity. The spacer thickness was continuously monitored during evaporation with an oscillating quartz unit. For supported lipid bilayer (SLB) measurements, a silica spacer of $10 \mathrm{~nm}$ was deposited on top of the graphene monolayer. 


\section{Sample preparation}

For single-molecule FLIM imaging, Atto 655 (ATTO-TEC GmbH, Siegen, Germany) molecules were used. Briefly, $1 \mathrm{nM}$ stock solution of Atto 655 was diluted to $100 \mathrm{pM}$ in Millipore water $\left(18.2 \mathrm{M} \Omega \mathrm{cm}\right.$ at $\left.25^{\circ} \mathrm{C}\right)$ and a $10 \mu \mathrm{l}$ aliquot of the same solution was spin-coated onto the substrates at $8000 \mathrm{rpm}$ for $50 \mathrm{~s}$. The fluorophore was particularly chosen for its well-known good photo-stability in air.

Supported lipid bilayers (SLBs) were prepared by vesicle fusion to the substrate as described in Ref. ${ }^{1}$ Small unilamellar vesicles (SUVs, diameter $\sim 50 \mathrm{~nm}$ to $100 \mathrm{~nm}$ ) composed of 1,2-dioleoyl-sn-glycero-3-phosphocholine (DOPC) or 1,2-dilauroyl-sn-glycero-3-phosphocholine (DLPC) were used (Avanti Polar Lipids, Alabama, USA). Lipids with fluorescently labelled head groups (Atto 655), namely 1,2-dipalmitoyl-sn-glycero-3-phosphoethanolamine (DPPE) and 1,2dilauroyl-sn-glycero-3-phosphoethanolamine (DLPE) were purchased (ATTO-TEC GmbH, Siegen, Germany). Unlabelled lipids were dissolved in chloroform at a concentration of $10 \mathrm{mg} / \mathrm{ml}$ whereas the fluorescently labelled lipids were diluted to $0.01 \mathrm{mg} / \mathrm{ml}$. For preparation of Atto 655-DPPEDOPC SLB, $1 \mu$ l of Atto 655-DPPE was mixed to $80 \mu$ of DOPC. The solution was dried in vacuum at $30^{\circ} \mathrm{C}$ for $1 \mathrm{~h}$ for evaporating the chloroform and obtaining a thin film. The film was then re-suspended with buffer solution (20 mM Tris-Cl, $100 \mathrm{mM} \mathrm{NaCl}, 10 \mathrm{mM} \mathrm{CaCl}_{2}, \mathrm{pH}$ 7.4), followed by thermomixing (Thermomixer Comfort, Eppendorf) for $1 \mathrm{~h}$ at $30^{\circ} \mathrm{C}$. After mixing, the solution was passed through a polycarbonate membrane with a pore diameter of $50 \mathrm{~nm}$ for extruding SUVs. The SUVs obtained were then deposited on the substrate and allowed to fuse for 1 hour to form a 

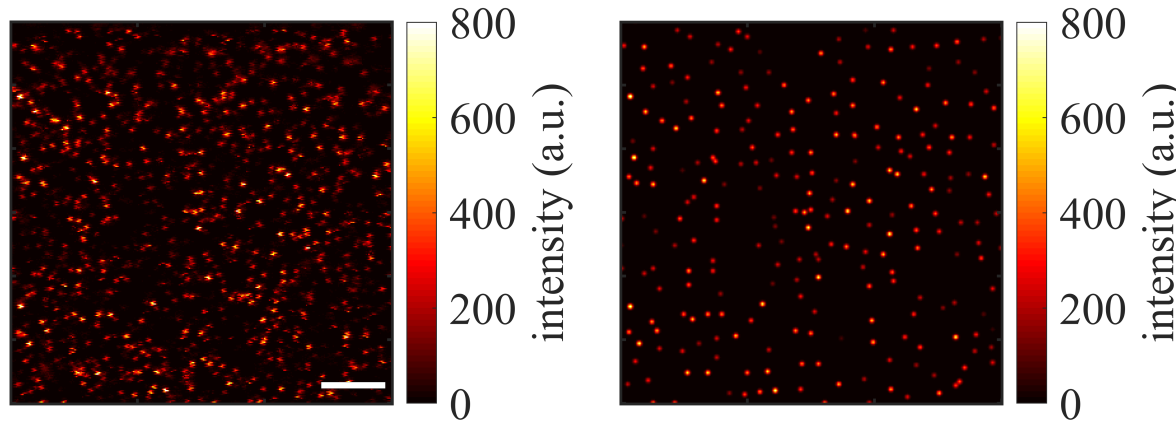

Figure S1: Confocal scan of spin-coated Atto 655 molecules on a $20 \mathrm{~nm}$ silica coated graphene surface. The left panel shows a $40 \mu \mathrm{m} \times 40 \mu \mathrm{m}$ raw area scan image obtained from the experiment. Right panel shows the same intensity image reconstructed after identifying single emitter pixels using a pattern matching algorithm. Scale bar corresponds to $7 \mu \mathrm{m}$.

uniform bilayer. The incubation was followed by a wash with the buffer solution ( $20 \mathrm{nM}$ Tris-Cl, $100 \mathrm{mM} \mathrm{NaCl}, 10 \mathrm{nM} \mathrm{CaCl}_{2}, \mathrm{pH} 7.4$ ) at least 20 times to remove unbound vesicles. The same protocol was followed for preparation of Atto 655-DLPE-DLPC bilayer.

Giant unilamellar vesicles (GUVs) were prepared by electro-formation in a custom built chamber. DOPC and Atto 655-DPPE were used to prepare GUVs. A lipid mixture of $98 \mu$ of DOPC and $2 \mu$ of Atto 655-DPPE in chloroform was deposited on the lower electrode plate and evaporated for 30 min under vacuum. The chamber was filled with $500 \mu \mathrm{l}$ of $300 \mathrm{mM}$ sucrose solution. Electroformation was performed for $3 \mathrm{~h}$ at $15 \mathrm{~Hz}$ alternating electric current (AC) and a peak-to-peak voltage of $1.6 \mathrm{~V}$ followed by $8 \mathrm{~Hz} \mathrm{AC}$ for $30 \mathrm{~min} .500 \mu \mathrm{l}$ of $300 \mathrm{mM}$ glucose was added to the chamber after the electroformation with simultaneously rinsing the electrode surface, the final suspension of GUVs were collected. 


\section{Fluorescence Lifetime Measurements}

Fluorescence lifetime measurements on surface immobilised dye molecules and supported lipid bilayers was performed using the commercial confocal setup Microtime 200 (PicoQuant GmbH, Berlin, Germany). ${ }^{2}$ The system is based on an Olympus IX-71 inverted microscope (Olympus Deutschland, Hamburg, Germany) amended with a side-port on the right side. For single-molecule FLIM measurements, areas of $40 \mu \mathrm{m} \times 40 \mu \mathrm{m}$ on the sample were scanned with a pixel dwelltime of $4 \mathrm{~ms}(100 \mathrm{~nm}$ effective pixel-size) with the aid of a three-axis piezo stage (P-562.3CD, Physik Instrumente, Karlsruhe, Germany). The excitation unit consists of a pulsed diode laser ( $\lambda_{\text {exc }}=640 \mathrm{~nm}$, LDH-D-C 640, PicoQuant) having a pulse width of $50 \mathrm{ps}$ FWHM and repetition rate of $40 \mathrm{MHz}$. Additionally, a clean-up filter (Z640/10, Chroma Technology) was used in the excitation path. A polarisation-maintaining single-mode optical fibre (PMC-400-4.2-NA010-3-APC250 V, Schäfter and Kirchhoff, Hamburg, Germany) was used to guide the laser light towards the microscope. The main optical unit consists of a fibre output that collimates the light into a beam of $12 \mathrm{~mm}$ diameter via an infinity-corrected $4 \mathrm{x}$ objective (UPISApo $4 \mathrm{X}$, Olympus). This beam was reflected by a quad-band dichroic mirror (Di01-R405/488/561/635, Semrock) towards the objective lens through the side port of the microscope. The laser power was maintained at $\sim 20 \mu \mathrm{W}$ at the back-focal plane of the objective lens. A high numerical aperture objective (UApoN 100X oil, 1.49 N.A., Olympus) was used to focus the light into the sample and was also used for collecting the fluorescence emission. Emission light was passed through the dichroic mirror and focused into a pinhole (diameter $100 \mu \mathrm{m}$ ) for confocal detection. After the pinhole, the light was refocused onto an avalanche photo diode ( $\tau$-SPAD, PicoQuant) using two achromatic lens doublets. In order to block 

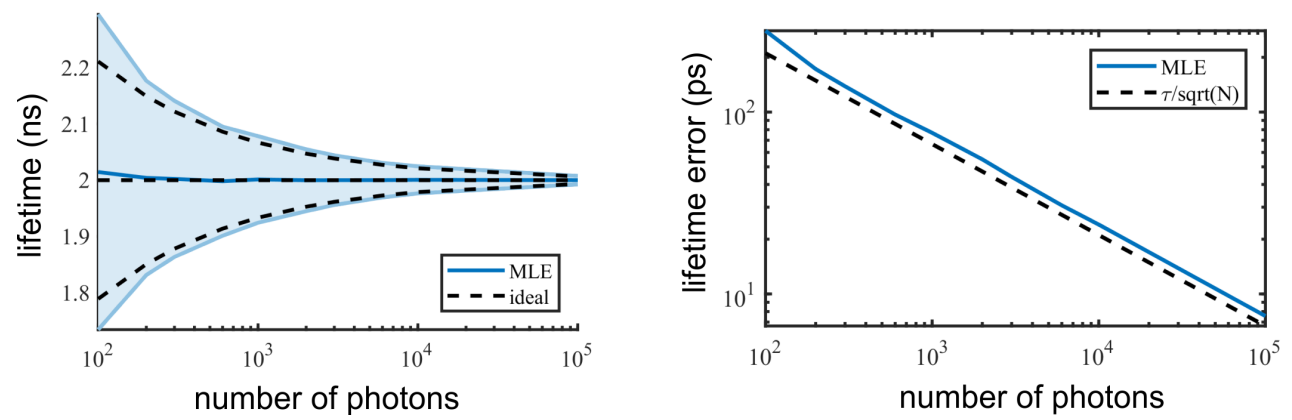

Figure S2: Fitted lifetime and its error. In the left panel, the lifetime is calculated from the average fitted lifetime (blue line) and displayed with its error calculated from the standard deviations (shaded area). The dashed lines mark the true lifetime and the ideal shot noise limit of $\tau / \sqrt{N}$. In the right panel, only the lifetime error is shown in a double logarithmic scale. The deviation from the ideal error is around $\sim 15 \%$.

back-scattered excitation light, a long-pass filter (BLP01-635R, Semrock) was used. Additionally, we used a band-pass filter (Brightline HC692/40, Semrock) before the detector. Dark count rate of the detector was less than 150 counts per second. Signals from the detector were processed by a multi-channel picosecond event timer (Hydraharp 400, PicoQuant) with 16 ps time resolution. ${ }^{3}$ Time-correlated single-photon counting histograms (TCSPC) were calculated from the timed photon signals. Figure S1 shows a typical scan image of fluorophores spin-coated on a $20 \mathrm{~nm}$ silica spacer on graphene.

\section{Lifetime Fitting}

For each molecule, a TCSPC histogram was computed from the recorded photons. A monoexponential decay function was fitted to the tail of the histogram ( $0.5 \mathrm{~ns}$ after the maximum) using a maximum likelihood procedure. The fitting function was

$$
I(t)=\frac{A}{\tau} \exp \left(-\frac{t}{\tau}\right)+b
$$


where $\tau$ is the fluorescence lifetime, $A$ the amplitude, and $b$ the background. The negative loglikelihood $\sum_{i} h\left(t_{i}\right) \log \left(I\left(t_{i}\right)\right)-I\left(t_{i}\right)$, where $h_{i}$ is the recorded TCSPC histogram and the sum runs over all TCSPC channels, was minimised using a Nelder-Mead simplex algorithm. Initial parameters for the optimisation were obtained by choosing the parameter set that minimises the least-squares error from a collection of exponential functions. We determined the fitting accuracy for our experiment with a Monte Carlo simulation, see Figure S2. We simulated TCSPC histograms with a signal-to-background ratio of 10 and a lifetime of $2 \mathrm{~ns}$. The exponential decay was convoluted with a Gaussian function as the instrumental response function (IRF) and the same cutoff was applied as for the data. Poissonian noise was applied to the histogram and the histogram was fitted as described above. This was repeated 1000 times. The mean of the fitted lifetimes is shown together with the standard deviation in Figure S2. We found no significant bias for our fitting procedure, even for as few as 100 photons. In the ideal case of a mono-exponential decay and no background, the lifetime can be estimated to a precision of $\tau / \sqrt{N}$ where $N$ is the number of recorded photons. Because our model estimates the amplitude and the background additionally, the lifetime error is $\sim 15 \%$ larger than for the ideal case. For example, the precision for fitting 1000 photons is $77 \mathrm{ps}$, which would allow for a localization precision of $<1 \AA$ (if the lifetime fitting would be the only source of error).

\section{Defocused imaging}

For determining the orientation of single molecules on a surface, we used defocused imaging. For that purpose, we spin-coated a $100 \mathrm{pM}$ solution of Atto 655 molecules on a substrate with $20 \mathrm{~nm}$ 
silica spacer on graphene. An emCCD camera (iXon DU-885K, Andor Technology, Ireland) was used for recording wide-field fluorescence images. A linearly polarised diode (PhoxX 647, 140 $\mathrm{mW}$, Omicron Laserage, Germany) with $\lambda_{\text {exc }}=647 \mathrm{~nm}$ was used for excitation. The laser beam was focused on the back aperture of objective lens (UApoN 100XOTIRF, 1.49 N.A., Olympus). The focused beam was shifted across the back aperture using a movable mirror in order to create a TIR illumination. Average laser intensity was $200 \mathrm{~W} / \mathrm{cm}^{2}$. Emission was collected by the same objective and then passed through the dichroic mirror (Di01-R405/488/561/635, Semrock) before being refocused onto the camera chip. The camera was cooled down to $-80^{\circ} \mathrm{C}$, preamp-gain was set to 3.7, and an em-gain of 20 was chosen. Experiments were done with an exposure time of ten seconds. In order to acquire defocused images, the objective was moved about $\sim 1 \mu \mathrm{m}$ towards the sample. Figure $2 \mathrm{c}$ in the main text shows defocused intensity patterns of Atto 655 molecules having an effective pixel size of $130 \mathrm{~nm}$. Calculation of model patterns for various orientations and defocus values, and data fitting with the computed patterns were done using the theory provided in Ref. ${ }^{4}$

\section{Dye orientation in GUVs}

Fluorescently labelled giant unilamellar vesicles (GUVs) were imaged on a home-built wide-field microscope. A linearly polarised laser beam $\left(\lambda_{\mathrm{exc}}=637 \mathrm{~nm}\right.$, OEM-SD-637-500) was used for excitation. Emission was imaged with an emCCD camera (Ixon Ultra 897, Andor Technology, Ireland). Experiments were performed while cooling the camera to $-70^{\circ} \mathrm{C}$, with an acquisition time of 1 second and an em-gain of 30. The underlying idea of this experiment is to identify high emission intensity areas in the image, which provides information about dye orientation with respect 
to the bilayer of the GUVs (see ${ }^{5}$ for details). Figure $3 b$ in the main text shows a typical GUV image, which shows that the dye molecules are oriented parallel to the bilayer surface.

\section{Conversion of lifetime values to distance values}

The theoretical background of calculating Metal-Induced Energy Transfer (MIET) curves for converting measured lifetime values into substrate-fluorophore distances is indicated in the main text, and all the technical details have already been published elsewhere. ${ }^{6,7}$ A MATLAB-based software package for the calculation of MIET lifetime-versus-distance curves as well as the conversion of lifetime data to distance maps, equipped with a graphical user interface, has been published free of charge at https://projects.gwdg.de/projects/miet.

While the published version of the software assumes that the dipole molecules are rotating quickly compared to their excited state lifetime, this was not the case for the measurements of the present work. For the measurements of single molecules spin-coated on graphene substrates with varying silica spacer thickness, the orientation distribution of immobilised molecules was taken into account as detailed in the main text. For evaluating the lifetimes of dyes in SLBs, a dye orientation parallel to the bilayer (and thus to the substrate) was assumed as described in the previous section.

\section{Multi-emitter localization on top of DNA origami using gMIET}

We used gMIET to co-localize axially two emitters attached to DNA origami at two different design heights. We designed a two-dimensional DNA origami structure ${ }^{8}$ with two DNA double-strands perpendicular to its surface. A further DNA strand, labelled with an Atto 647N fluorophore, was 


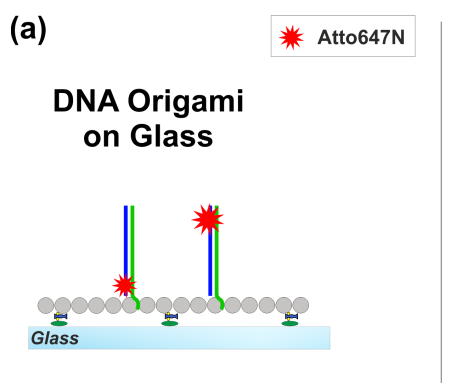

(b1)

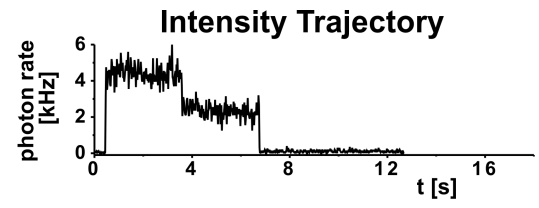

(b2)
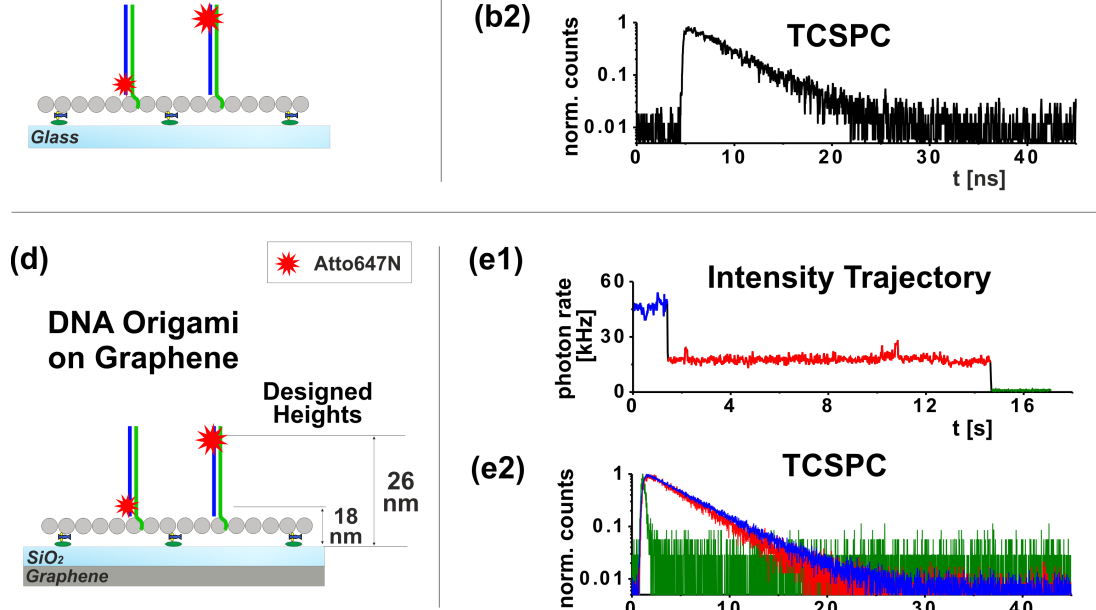

(e1)

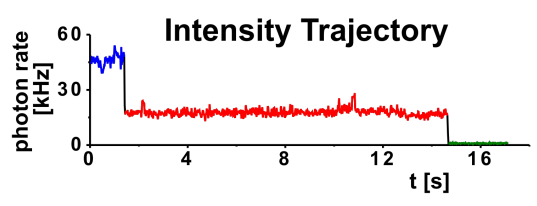

(e2)

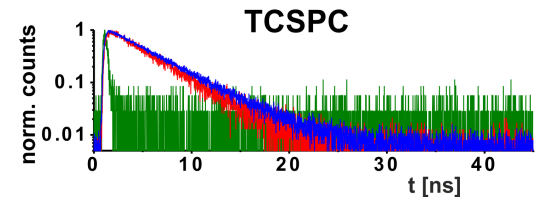

(c)
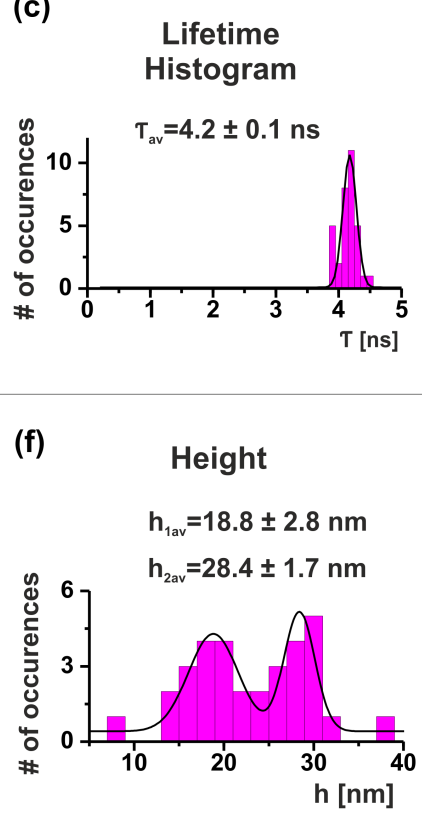

Figure S3: Co-localization measurement of two Atto 647N fluorophores on DNA origami. (a-c) Reference measurement of DNA Origami structures on top of a glass surface. (a) DNA origami design: two Atto 647N molecules are attached to the structure at different heights. (b1) Representative single-molecule intensity time trace. (b2) Normalised TCSPC histogram of the same molecule. (c) Lifetime histogram of 33 doubly-labelled DNA origami structures. (d-f) DNA origami structures measured on top of graphene $\left(\mathrm{SiO}_{2}\right.$ spacer thickness is $\left.5 \mathrm{~nm}\right)$. (e1) Representative single-molecule intensity time. (e2) TCSPC histograms of the emitters. The colours correspond to the bleaching steps in time trace. (f) Height histogram of 29 doubly-labelled DNA origami structures. 
designed to hybridise against two elongated DNA origami staples, in the opposite directions, as shown on Figure S3a. In this way we placed the emitter at a height difference of about $8 \mathrm{~nm}$ from each other. The DNA origami was attached to the surface using biotin-avidin. During the experiment, a low coverage of molecules on the surface was used to be able to distinguish between individual origami constructs. Data for individual origami constructs were acquired manually point-by-point, until total photo-bleaching of the attached fluorophores. For this purpose, areas of $40 \mu \mathrm{m} \times 40 \mu \mathrm{m}$ were initially scanned for identifying and localizing single structures, and then point-by-point lifetime and intensity measurements were performed. Blinking of DNA-bound Atto $647 \mathrm{~N}$ fluorophores was suppressed by addition of Trolox $2 \mathrm{mM}$ into the imaging buffer (Tris $10 \mathrm{mM}$, EDTA $1 \mathrm{mM}, \mathrm{NaCl} 40 \mathrm{mM}$ ). Two-step bleaching was observed in at least 80 percent of the measured structures. A typical time trace and TCSPC histogram are shown in Figure S3b. For reference, similar measurements were done on glass cover-slides with no graphene/ $/ \mathrm{SiO}_{2}$. In the lifetime histogram built from many individual measurements, a single peak is found at $(4.2 \pm 0.1) \mathrm{ns}$, Figure 3c. Then, DNA origamis were immobilised using an identical procedure on top of $5 \mathrm{~nm}$ silica coated graphene, Figure S3d. As expected, the intensity of the lower emitter was more quenched then that of the upper one. A typical time trace is shown on Figure S3e1. Step-wise bleaching analysis of the lifetimes of two emitters was performed as described previously ${ }^{9}$. Briefly, bleaching steps were identified and the corresponding TCSPC histograms were calculated, as shown in Figure S3e1-2. The colours of the TCSPC histograms in Figure S3e 2 correspond to the colours of the time trace patches between bleaching steps as shown in Figure S3e1. In order to extract lifetime values, we first fit the TCSPC histogram for one single emitter (red curve), and afterwords perform a bi-exponential 
fit for the TCSPC histogram of two emitters (blue curve), while we fixed the obtained lifetime value from the single emitter fit. All the fitted lifetime values were then converted into height values, see Figure S3f. Two peaks are visible at $(18.8 \pm 2.8) \mathrm{nm}$ and $(28.4 \pm 1.7) \mathrm{nm}$. The height for the lower emitter agrees with the design value, while the height of the upper emitter slightly deviates from its design value. This is possibly due to a tilt of the DNA origami structure, as seen before for similar structure which were attached to a surface ${ }^{9}$.

\section{Axial localization of single Atto 488 molecules using gMIET}

To demonstrate that gMIET works also well in the blue-green spectral region, we localize single Atto 488 molecules spin-coated on graphene coated with $10 \mathrm{~nm}$ thick $\mathrm{SiO}_{2}$. We measured the fluorescence lifetime of the fluorophores following the same procedure as described for Atto 655 . Again, we used defocused imaging for determining emission dipole inclination angles of the molecules. The gMIET calibration curve for Atto 488 was then calculated by using the free-space lifetime, quantum yield, and orientation information. Free space lifetime $\tau_{0}=3.6 \mathrm{~ns}$ and quantum yield $\phi=0.74$ were taken from a previously published report ${ }^{10}$. We obtain an axial distance of $12 \pm 2 \mathrm{~nm}$, where the bias of $\sim 2 \mathrm{~nm}$ originates most likely from surface roughness and quantum yield variations of adsorbed molecules. Lifetime and orientation distributions along with the gMIET calibration curve and height distribution are presented in figure S4. 
(a)

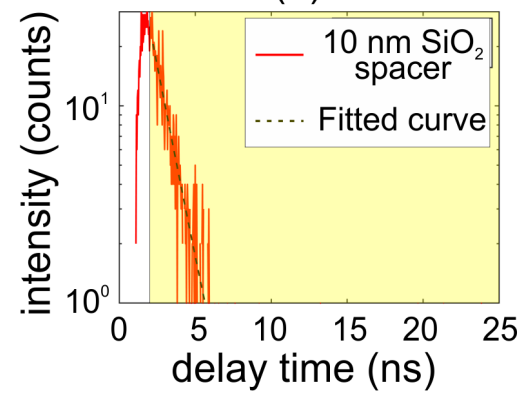

(c)

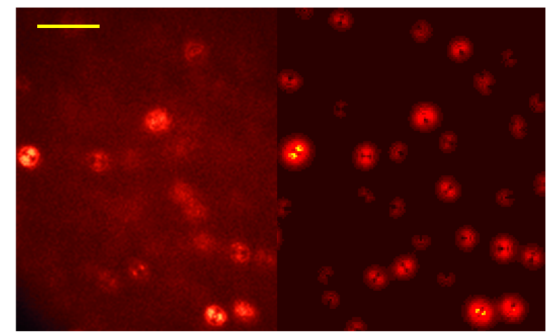

(e)

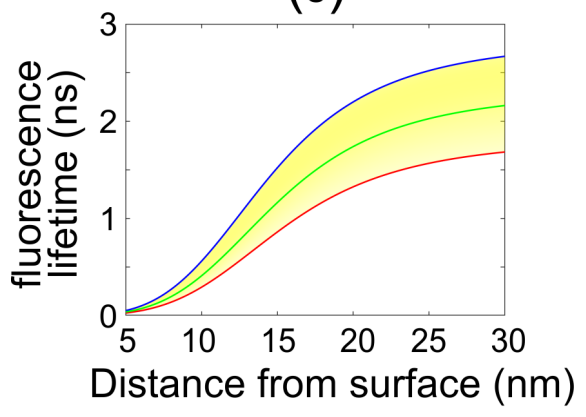

(b)

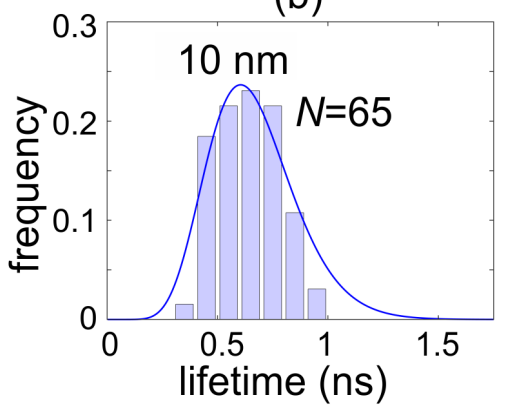

(d)

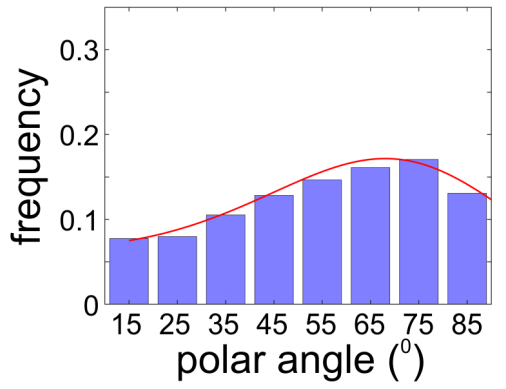

(f)

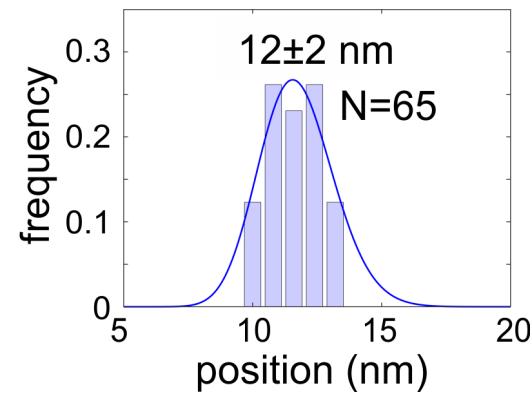

Figure S4: Axial localization of single Atto 488 molecules using gMIET (a) TCSPC histogram for a single Atto 488 emitter. The shaded region is used for a maximum likelihood mono-exponential tail fit of the fluorescence decay time. (b) Lifetime distributions as obtained by tail-fitting the decay curves of individual molecules. Numbers above a distribution indicate how many molecules contributed to the corresponding distribution. Solid lines represent fits of a general Gamma distribution to the histograms. (c) Measured defocused single molecule images (left) for Atto 488 molecules deposited on $\mathrm{SiO}_{2}$, and theoretically fitted defocused images (right). Scale bar is $1 \mu \mathrm{m}$. (d) Bar histogram of molecular orientation distribution (angle towards the vertical axes) as extracted from the defocused images shown in (c). (e) Calculated gMIET calibration curves: Blue and red curves show the gMIET calibration curves which one would have for purely horizontally or vertically oriented molecules. The yellow density plot shows a distribution of gMIET curves where the shading reflects the weight proportional to the orientation distribution from panel (d). The green curve is the corresponding orientation-averaged gMIET calibration curve used for subsequently converting experimental lifetime to distance values. (f) Distance distributions as obtained by converting the lifetime values from panel (b) into distance values by using the green gMIET calibration curve from panel (e). 


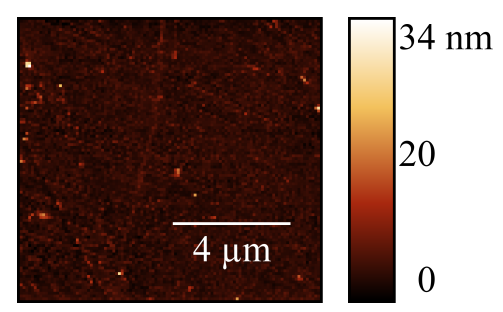

Figure S5: AFM image of a graphene surface coated with $15 \mathrm{~nm} \mathrm{SiO}_{2}$. In this case, we obtain a root-mean-square value of roughness as $1.8 \mathrm{~nm}$. AFM scans were performed on the same substrate on which single molecule fluorescence lifetime measurements on Atto 655 were done. 
1. Richter, R. P., Bérat, R. \& Brisson, A. R. Formation of solid-supported lipid bilayers: an integrated view. Langmuir 22, 3497-3505 (2006).

2. Buschmann, V., Krämer, B., Koberling, F., Macdonald, R. \& Rüttinger, S. Quantitative fcs: determination of the confocal volume by fcs and bead scanning with the microtime 200 . Application Note PicoQuant GmbH, Berlin (2009).

3. Wahl, M. et al. Scalable time-correlated photon counting system with multiple independent input channels. Review of Scientific Instruments 79, 123113 (2008).

4. Patra, D., Gregor, I. \& Enderlein, J. Image analysis of defocused single-molecule images for three-dimensional molecule orientation studies. The Journal of Physical Chemistry A 108, 6836 (2004).

5. Bagatolli, L. A. To see or not to see: lateral organization of biological membranes and fluorescence microscopy. Biochimica et Biophysica Acta (BBA)-Biomembranes 1758, 15411556 (2006).

6. Karedla, N., Ruhlandt, D., Chizhik, A. M., Enderlein, J. \& Chizhik, A. I. Metal-induced energy transfer. In Advanced Photon Counting, 265-281 (Springer, 2014).

7. Karedla, N. Single-Molecule Metal-Induced Energy Transfer: From Basics to Applications (Springer, 2017).

8. Tomov, T. E. et al. Dna bipedal motor achieves a large number of steps due to operation using microfluidics-based interface. ACS Nano 11, 4002-4008 (2017). 
9. Isbaner, S. et al. Axial colocalization of single molecules with nanometer accuracy using Metal-Induced Energy Transfer. Nano Letters 18, 2616-2622 (2018).

10. Chizhik, A. I., Gregor, I., Ernst, B. \& Enderlein, J. Nanocavity-based determination of absolute values of photoluminescence quantum yields. ChemPhysChem 14, 505-513 (2013). 


\subsection{Manuscript: Graphene- and metal-induced en- ergy transfer : from single-molecule imaging to live cell nanoscopy with (sub)-nanometer axial resolution}

In this protocol, I present a detailed workflow for GIET and MIET imaging. We discuss previously published experimental results of the methods $[22,24]$ and present a detailed description of the instrumentation, sample preparation, data acquisition and analysis, in a step-by-step manner. We believe that this protocol will provide a an easy-to use recipe for performing MIET and GIET experiments, which will make the techniques much more accessible to the scientific community.

This invited protocol is currently under revision. The protocol will be published as "Arindam Ghosh, Alexey I. Chizhik, Narain Karedla and Jörg Enderlein. Grapheneand metal-induced energy transfer : From single-molecule imaging to live cell nanoscopy with (sub)-nanometer axial resolution. Nature Protocols". A.G. co-wrote the manuscript with inputs from other authors. A.G. generated figures 1,2 and 3. Figure 4 and 5 were taken and reproduced with permission from the previously published original report on GIET [24]. Figures 6, 7 and 8 were adapted and reproduced with permission from the original research article on MIET imaging [22]. 


\title{
Graphene- and Metal-Induced Energy Transfer:
}

From single-molecule imaging to live cell nanoscopy with (sub)-nanometre axial resolution Arindam Ghosh $^{1}$, Alexey I. Chizhik ${ }^{1}$, Narain Karedla ${ }^{3}$, and Jörg Enderlein ${ }^{1,2^{*}}$

\footnotetext{
${ }^{1}$ Third Institute of Physics - Biophysics, Georg August University, Friedrich-Hund-Platz 1, 37077 Göttingen, Germany.

${ }^{2}$ Cluster of Excellence "Multiscale Bioimaging: from Molecular Machines to Networks of Excitable Cells” (MBExC), Georg August University, Göttingen, Germany.

${ }^{3}$ Physical and Theoretical Chemistry, University of Oxford, Oxford, UK

${ }^{*}$ Correspondence should be addressed to J.E. (jenderl@gwdg.de)
}

\begin{abstract}
Super-resolution fluorescence imaging that surpasses the classical optical resolution limit is widely utilized for resolving the spatial organization of biological structures at molecular length scales. Among the most commonly used super-resolution methods are STED [1] and singlemolecule localization based super-resolution microscopy (SMLM) techniques such as photoactivated localization microscopy (PALM) [2], stochastic optical reconstruction microscopy (STORM) [3], fluorescent PALM (fPALM) [4], direct STORM (dSTORM) [5], or point accumulation for imaging in nanoscale topography (PAINT) [6, 7]. For achieving superresolution in SMLM also along the optical axis, several schemes such as bi-plane imaging [8], astigmatic imaging [9], or wavefront shaping [10] have been developed and successfully applied for the study of 3D biological structures. Nonetheless, the achievable axial localization accuracy is typically three to five times worse than the lateral localization accuracy. The only exceptions are interferometric methods such as iPALM [11, 12] or isoSTED [13, 14], but for the price of enormous technical complexity. Recently, metal-induced energy transfer (MIET) imaging was developed for localizing fluorophores along the axial direction with nanometre accuracy, using only a conventional fluorescence lifetime imaging microscope (FLIM) [15]. Recently, we demonstrated that replacing the metal layer with graphene (graphene-induced energy transfer or GIET) [16] enables sub-nanometre optical localization. Here, we provide a step-by-step protocol for MIET and GIET imaging in single molecules, supported lipid bilayer and live cell membranes.
\end{abstract}




\section{Introduction}

Most of the SMLM methods exploit switching of fluorescent emitters between fluorescent on-states and non-fluorescent off-states. One records consecutive images with different sub-set of molecules switched into a fluorescent on-state such that in each image the fluorescing molecules are spatially separated. In a subsequent step, images of individual molecules are identified and localized with high precision. By repeating this for many different subsets of molecules, on consecutively accumulates a final image of single molecule localizations, representing the sample with a spatial resolution much superior to the classical resolution limit. The physical basis of this approach is the fact that the centre position of a single molecule's image can be determined with a higher accuracy than the size of the image itself (latter is determined by the optical resolution of the used microscope). An alternative method, MINFLUX [17] was developed recently where the position of a fluorophore is probed by a scanning focus with zero intensity at the centre. For that purpose, MINFLUX uses a donut-shaped excitation beam profile (similar to the donut beam used in stimulated emission depletion (STED) microscopy [1]) and determines the position of the emitter with respect to the centre of the donut where it will not be excited. MINFLUX achieves a localization precision of $\sim 1 \mathrm{~nm}$ at much lower photon budget than usually required by all conventional SMLM methods. Recently, 3D MINFLUX demonstrated such a resolution even along the optical axis [18]. However, similar to iPALM or isoSTED, MINFLUX is a technically complex method that currently limits its wider application.

We introduced MIET imaging as a rather simple alternative for optical localization of fluorophores along the third dimension. The underlying principle of MIET is based on an electromagnetic-fieldmediated energy transfer from an optically excited fluorescent emitter (donor) to a thin planar metal film (acceptor). The effect of the energy transfer is manifested by a distance-dependent modulation of an emitter's fluorescence lifetime. The energy transfer from the excited state to the planar metal film is a predominantly a near-field effect and very similar to Förster resonance energy transfer (FRET). However, unlike FRET, the planar geometry of the acceptor leads to a monotonous relation between lifetime and distance up to $\sim 150 \mathrm{~nm}$ from the metal layer, i.e. over an interaction range that is ca. ten times larger than that of typical FRET. Since the energy transfer rate depends on the distance of an emitter from the metal layer, fluorescence lifetime values can be directly converted into axial distance values with nanometre accuracy $[19,20,21,22,23,24,25,26]$. Recent studies have shown that using materials such as indium-tin oxide (ITO) or graphene as the quenching layer reduces the interaction range, while increasing proportionally the axial resolution [27]. We have shown that by replacing the 
metal with a graphene monolayer, the interaction range comes down to $\sim 25 \mathrm{~nm}$, and consequently the localization accuracy of MIET is improved by nearly an order of magnitude [16]. In this Protocol, we provide a detailed description of the workflow of MIET measurements both with metal and graphene as quenchers. Firstly, we provide a theoretical basis for the quantitative understanding of MIET and GIET. Secondly, we present a detailed step-by-step protocol for exemplary MIET and GIET experiments and data analysis.

\section{Principle of MIET and GIET}

Depending on the desired maximal resolution and required dynamic distance range, one selects either graphene (GIET) or metal (MIET) coating for a substrate. The general sample geometry of (g)MIET experiments is depicted in Fig. 1A. A substrate consists of a thin semi-transparent metal film or a graphene layer deposited on top of a cleaned glass coverslip. An additional thin transparent silica substrate is deposited on top of the quenching layer which prevents direct contact with fluorescent molecules and protects the layers beneath from chemical or physical damage. For the current work, we used for MIET a $10 \mathrm{~nm}$ gold film sandwiched between $2 \mathrm{~nm}$ and $1 \mathrm{~nm}$ titanium layers above and below, evaporated on top of a standard glass coverslip (thickness $=170 \mu \mathrm{m}$, refractive index $=1.52$ ). For GIET, a graphene monolayer (thickness of $0.34 \mathrm{~nm}$ ) is used instead of the metal layers. The thickness of the silica spacer on top depends on the desired application, as we will be demonstrated on several examples below. We will refer to this layered substrate as MIET/GIET substrate for the remainder of this article. Excitation and detection of fluorescence is done through this substrate from beneath with a high numerical aperture objective. The near-field coupling of a fluorescing molecule with the MIET substrate has been modelled and described in detail in several publications [25, 26]. The fluorescing molecule is modelled as an ideal electric dipole emitter positioned at a distance $z_{0}$ from the substrate. Its electromagnetic field can be mathematically represented by a superposition of plane electromagnetic waves. The interaction of each of these waves with the substrate is described by Fresnel's reflection and transmission laws, thus allowing for calculating the full field as a superposition of direct, reflected and transmitted plane waves. The full emission rate of the molecule is then calculated by integrating the Poynting vector of the full field over a closed surface enclosing the molecule. This leads to an expression for the radiative emission power, 
$S\left(\theta, z_{0}\right)$ of the molecule as a function of its distance $z_{0}$ and orientation described by the angle $\theta$ between its emission dipole axis and the vertical axis:

$$
S\left(\theta, z_{0}\right)=S_{\perp}\left(z_{0}\right) \cos ^{2} \theta+S_{\|}\left(z_{0}\right) \sin ^{2} \theta
$$
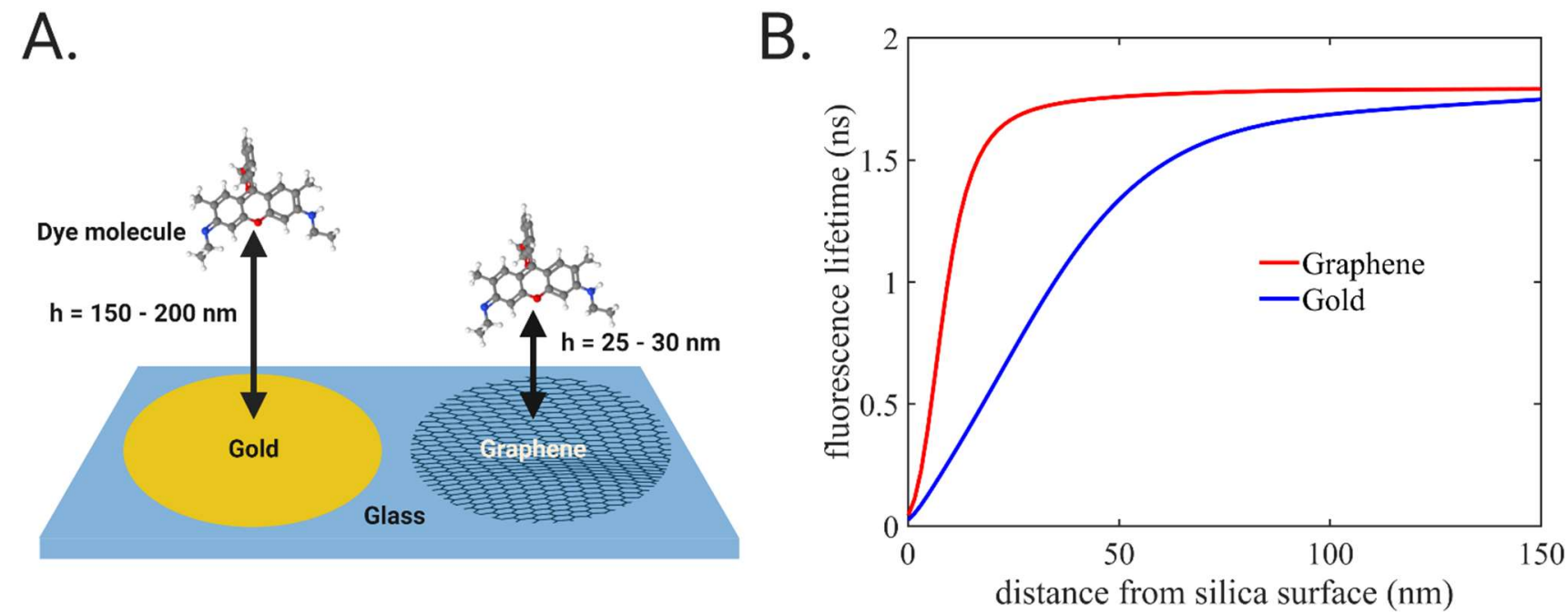

Figure 1: Working principle of MIET and GIET. A. The substrate consists of titan/gold/titan multilayer or a single graphene sheet on a glass coverslip, covered by a protecting silica layer (not shown). A fluorescent molecule within a range of up to ca. $150-200 \mathrm{~nm}$ (for metal) or 25 $30 \mathrm{~nm}$ (for graphene) can electromagnetically couple to the layers which leads to a distancedependent quenching of its fluorescence in the visible spectral range. B. Fluorescence lifetime as a function of distance of a molecule from the surface of a silica spacer of $5 \mathrm{~nm}$ thickness deposited on gold (blue) or graphene (red) layer. Calculations were performed for Atto655 for a single emission wavelength of $680 \mathrm{~nm}$. The refractive index of the upper medium was set to 1.33 (water), and the refractive index of glass was set to 1.52 .

where $S_{\perp}\left(z_{0}\right)$ and $S_{\|}\left(z_{0}\right)$ are the radiative emission rates of a dipole emitter oriented perpendicular and parallel to the substrate, respectively. This emission power $S\left(\theta, z_{0}\right)$ is directly related to the molecule's radiative transition rate $k_{r}$ from its excited to its ground state. However, most fluorescent molecule can also return to their ground state non-radiatively with rate $k_{n r}$, which is determined by their quantum yield (QY) $\varphi=k_{r} /\left(k_{r}+k_{n r}\right)$ that gives the ratio between emitted photons to number of excitations. Taking into account this non-radiative transitions, the final expression for the excitedstate fluorescence lifetime $\tau_{f}\left(\theta, z_{0}\right)$ reads:

$$
\frac{\tau_{f}\left(\theta, z_{0}\right)}{\tau_{0}}=\frac{S_{0}}{\varphi S\left(\theta, z_{0}\right)+(1-\varphi) S_{0}}
$$


Here, $\tau_{0}$ is the free-space lifetime in the absence of MIET, and $S_{0}$ is the free-space emission power of an ideal electric dipole emitter that is given by $S_{0}=c n k_{0}^{4} p^{2} / 3$, with $c$ being the vacuum speed of light, $k_{0}$ the wave vector in vacuum, $n$ the refractive index of the solvent (water), and $p$ the amplitude of the emission dipole moment vector. The model takes into account all the optical properties of the metal/graphene substrate (thickness and wavelength-dependent complex-valued refractive index; refractive index of the cover slide), and also the photophysics of the fluorophore (emission spectrum, free excited state lifetime, free quantum yield, but also orientation with respect to the substrate). Figure $1 \mathrm{~B}$ shows the calculated relative lifetime $\tau_{f} / \tau_{0}$ of as a function of distance $z_{0}$. Here, we have assumed that the emitter is free to rotate on a time scale much faster than the excited state lifetime so that the emission power $S\left(\theta, z_{0}\right)$ has to be averaged over all possible orientations. The thickness of the silica layer above the MIET substrate was set to $5 \mathrm{~nm}$ and the quantum yield values were taken from the product sheet of the supplier (ATTO-TEC GmbH).

\section{Characterization of fluorophores:}

\section{Orientation of fluorophores}

The assumption of rapidly rotating dipole emitters is only correct if the fluorophore is rotating on a timescale much shorter than its excited state decay time. This is mostly the case when dyes are tagged to biomolecules of interest via long flexible linkers. However, for immobilized molecules one needs to determine their dipole orientation for correctly converting measured lifetime values into a distance of a molecule from the substrate. Fig. 2B presents the distance-to-lifetime dependence for an Atto655 dye molecule with fixed orientation (either parallel or orthogonal with respect to the surface). Curves for all other possible orientations will fall into the shaded region in between these two extreme cases. One efficient way to experimentally determine the three-dimensional orientation of immobilized molecules is defocused imaging $[\mathbf{2 8}, \mathbf{2 9}, \mathbf{3 0}]$. Using the distribution of emission dipole inclination angles $\theta$ as obtained from such defocused imaging, we calculate the orientation-averaged MIET calibration curve as

$$
\left\langle\tau_{f}\left(z_{0}\right)\right\rangle=\int_{0}^{\pi / 2} d \theta \sin \theta \rho(\theta) \tau_{f}\left(z_{0}, \theta\right)
$$


A.

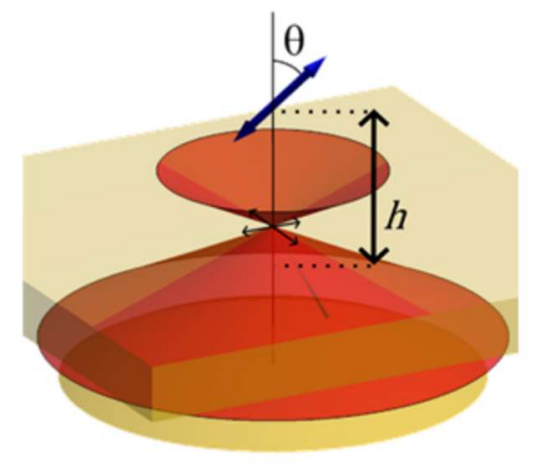

B.

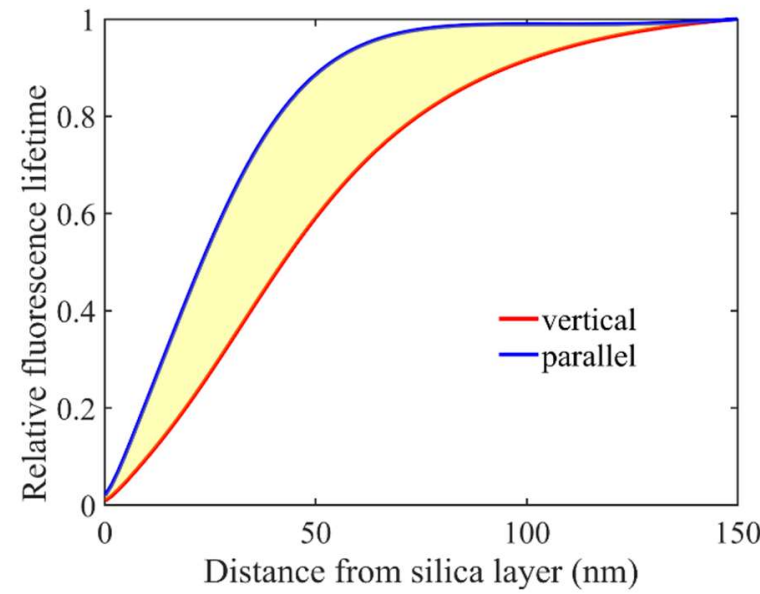

C.

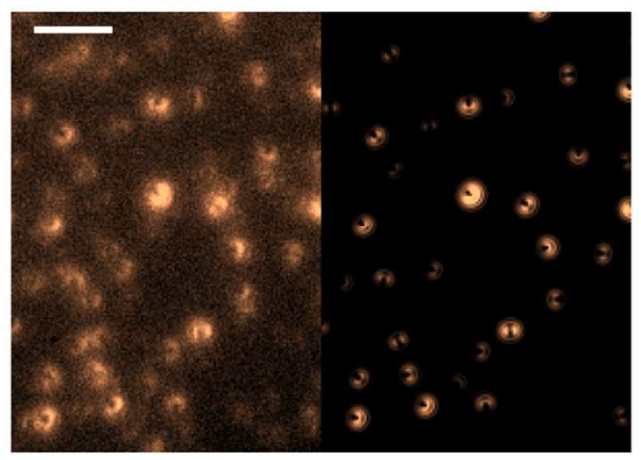

D.

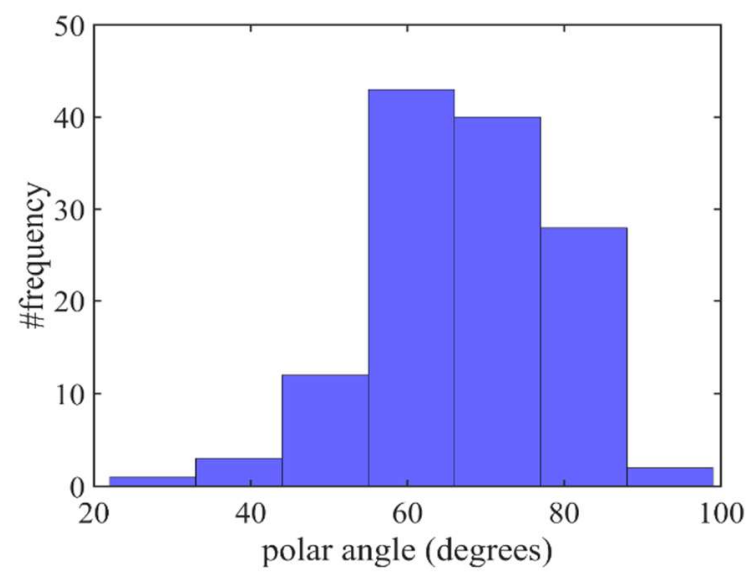

Figure 2: Orientation of fluorescent emitters. A. Detection scheme of a dipole emitter through a gold film at height $\boldsymbol{h}$ from the surface and with orientation angle $\boldsymbol{\theta}$ to the normal. B. Calculated MIET calibration curve for Atto655 for two extreme orientations, orthogonal (red) and parallel (blue) to the normal. Calculations were done at an emission wavelength of $680 \mathrm{~nm}$ and setting the refractive index of the upper medium to 1 (air) and that of glass to 1.52. Fluorescent molecules were immobilized on top of the $5 \mathrm{~nm}$ silica spacer deposited on a $10 \mathrm{~nm}$ gold layer. $C$. Defocused images of single Atto655 molecules that were spin-coated on the MIET/GIET substrate. Left panel shows a recorded image, and right panel shows the theoretical fit. Scale bar $1 \mu \mathrm{m}$. D. Distribution of polar angles as obtained from $\mathrm{C}$. This orientation distribution is used to calculate an orientation-averaged MIET/GIET curve.

where $\rho(\theta)$ denotes the orientation probability distribution of the dipoles. 


\section{Quantum yield of fluorophores}

In order to calculate a MIET calibration curve, one requires a priori knowledge of the quantum yield (QY) of the used fluorophores. Absolute QY values can be measured using any existing method, such as comparison to a reference sample, and integrating sphere, or a nanocavity-based method [31]. The advantage of the nanocavity method is that it is insensitive to the presence of non-luminescent impurities within a sample, that it is reference-free, and that it requires only minute amounts of sample (few microliters of low-concentration fluorophore solution). Similar to MIET, it uses the electromagnetic coupling of a fluorophores excited state to the electromagnetic modes in a cavity of variable size. By recording the fluorescence lifetime as a function of cavity size, one obtains a characteristic lifetime modulation curve which modulation depth is determined by the fluorophore's quantum yield. Thus, such a measurement can be used to directly calculate an unknown QY value. Details of the method has been described in many publications, see e.g. [31, 32]. Table 1 provides the quantum yields of some fluorophores that have been used for MIET experiments. Many dye providers report about the quantum yields of their probes. However, one should keep in mind that labelling biomolecules such as proteins or lipids can alter the fluorescent lifetime and quantum yields of a fluorescent molecule. In that case one is advised to independently measure the QY under conditions as close as possible to those used in the final MIET/GIET experiments

\section{Choice of fluorescent labels}

For MIET/GIET experiments, one can use a wide range of fluorescent emitters. In principle, any bright, photostable fluorescent label with a mono-exponential fluorescence decay and a reasonably long fluorescence lifetime (more than $\sim 1 \mathrm{~ns}$ ) can be used. We recommend longer lifetime fluorescent dyes due to the simplicity of tail-fitting of TCSPC histograms for determining fluorescence lifetime values, as compared to a full deconvolution of TCSPC histograms that requires the measurement of an instrumental response function (IRF). A list of fluorophores which have already been utilized for MIET is provided in Table 1. However, when a dye is tagged to a protein, a nucleic acid, or a lipid, it is recommended to re-measure the quantum yield and free-space fluorescence lifetime. For single molecule experiments, Atto655 NHS ester was used. GIET experiments on SLBs were performed using Atto655 labelled lipid head groups [16]. Cell mask deep red plasma membrane stain was used for profiling the basal membrane of living cells [15]. 


\begin{tabular}{|c|c|c|c|c|}
\hline Fluorophore & $\begin{array}{c}\text { Excitation } \\
\text { maximum (nm) }\end{array}$ & $\begin{array}{c}\text { Emission } \\
\text { maximum (nm) }\end{array}$ & $\begin{array}{c}\text { Fluorescence } \\
\text { lifetime in water } \\
\text { (ns) }\end{array}$ & Quantum yield \\
\hline Atto 488 & 498 & 526 & 4.1 & 0.80 \\
\hline Alexa Fluor 488 & 490 & 525 & 4.1 & 0.92 \\
\hline Rhodamine 6G & 532 & 550 & 4.1 & 0.99 \\
\hline Alexa Fluor 546 & 556 & 573 & 4.1 & 0.79 \\
\hline Alexa Fluor 633 & 621 & 637 & 3.2 & 0.59 \\
\hline Atto 647N & 646 & 664 & 1.8 & 0.30 \\
\hline Atto655 & 663 & 680 & 1.4 & 0.28 \\
\hline Cell mask deep & 650 & 668 & & \\
\hline $\begin{array}{c}\text { red plasma } \\
\text { membrane stain }\end{array}$ & & & & \\
\hline
\end{tabular}

\section{Choice of substrate and wavelength dependence}

A comparison of MIET and GIET quenching is presented in Fig. 1B, which shows the distancedependent lifetime modulation of Atto655 in air near graphene and near a gold layer, with a $5 \mathrm{~nm}$ silica spacer on top in both cases. The exact modification of the emission rate of a dye molecule depends on several factors such as the refractive index and thickness of the metal film, the layers and thicknesses the media above and below, the emission wavelength, and the quantum yield of the dye. Depending on the requirements of an experiment (axial resolution desired, maximum height range, refractive index of the medium) and dye characteristics one needs to calculate a MIET calibration curve in order to find the most suitable wavelength range and metal film thickness. In general, the thicker the metal film, the larger are photon losses due to increased absorption in the metal layer. Although metals such as aluminium, copper etc. can be used, we generally restrict ourselves to gold or silver due to their ability to quench up to $\sim 200 \mathrm{~nm}$ for fluorophores in the red spectral region (emission $\sim 690 \mathrm{~nm}$ ) and lower absorption losses. The choice of wavelength also plays an important role while designing a MIET/GIET experiment. The lifetime-distance dependence is steeper at shorter wavelengths than at longer wavelengths. For example, for a dye with a QY in the range between 0.3 and 1, the MIET calibration curve shows a monotonous increase in lifetime up to a distance of about $60 \mathrm{~nm}$ at green 
wavelengths $(\sim 520 \mathrm{~nm})$, whereas for far red wavelengths $(\sim 700 \mathrm{~nm})$, efficient quenching is observed up to 150 to $200 \mathrm{~nm}$. For single-molecule MIET (smMIET), the range of quenching and localization accuracy are inversely related. Most fluorescent dyes have an excited state lifetime $\tau_{f}$ between 1 and $5 \mathrm{~ns}$. The precision of lifetime measurements depends on the number of collected photons. Assuming a pure Poissonian statistics, the error of lifetime estimation is given by

$$
\Delta \tau \geq \frac{\tau_{f}}{\sqrt{N}}
$$

where $N$ is the total number of photons collected from a molecule before photobleaching or blinking. Equality occurs only for ideal background-free conditions. For example, if one collects $\sim 1000$ photons, the relative error of a determined lifetime value cannot be smaller than 0.03 . Using the MIET curves, this error can be translated into an axial error of distance determination. In smMIET, one collects fewer photons than in other single-molecule imaging experiments, due to the absorption and reflective loses arising from the thin metal film. Assuming that the relative brightness of a molecule is proportional to its fluorescence lifetime, the axial localization error is larger if a molecule is closer to the metal surface. In contrast to all metal substrates, a graphene monolayer has unique optical properties that lead to an efficient quenching range of only $\sim 25-30 \mathrm{~nm}$. Due to this short interaction range, GIET can achieve an axial localization accuracy below one nanometre.

In the next section, we explain in detail each step of a MIET/GIET measurement workflow. This includes the experimental requirements for MIET/GIET, in particular, the description of the fluorescence microscope, MIET/GIET substrate preparation, choice of fluorophores, fluorescent labelling strategies, and sample preparation. We will discuss this all for three exemplary applications: (a) single molecule localization with GIET; (b) GIET measurements of supported lipid bilayers (SLBs); and (c) MIET imaging of live cells for mapping their basal membrane.

\section{Experimental design}

\section{Fluorescence microscope setup}


All MIET experiments presented here were done with a homebuilt confocal microscope equipped with a multichannel picosecond event timer (HydraHarp 400, PicoQuant $\mathrm{GmbH}$ ) for time-correlated singlephoton counting (TCSPC). Fig. 3B illustrates the experimental setup where a supported lipid bilayer (SLB) with fluorophore-tagged head groups is placed on a GIET substrate. A high numerical aperture objective (Apo N, 100×/1.49 NA oil immersion, Olympus) is used for both focusing excitation light through the substrate and collecting the fluorescence light (epi-fluorescence microscope). For excitation, a pulsed white-light laser (SC400-4-20, Fianium) with an acousto-optical tunable filter (AOTFnC-400.650-TN, AA Optic) is employed. The tunable filter is used to select the desired wavelength for excitation.

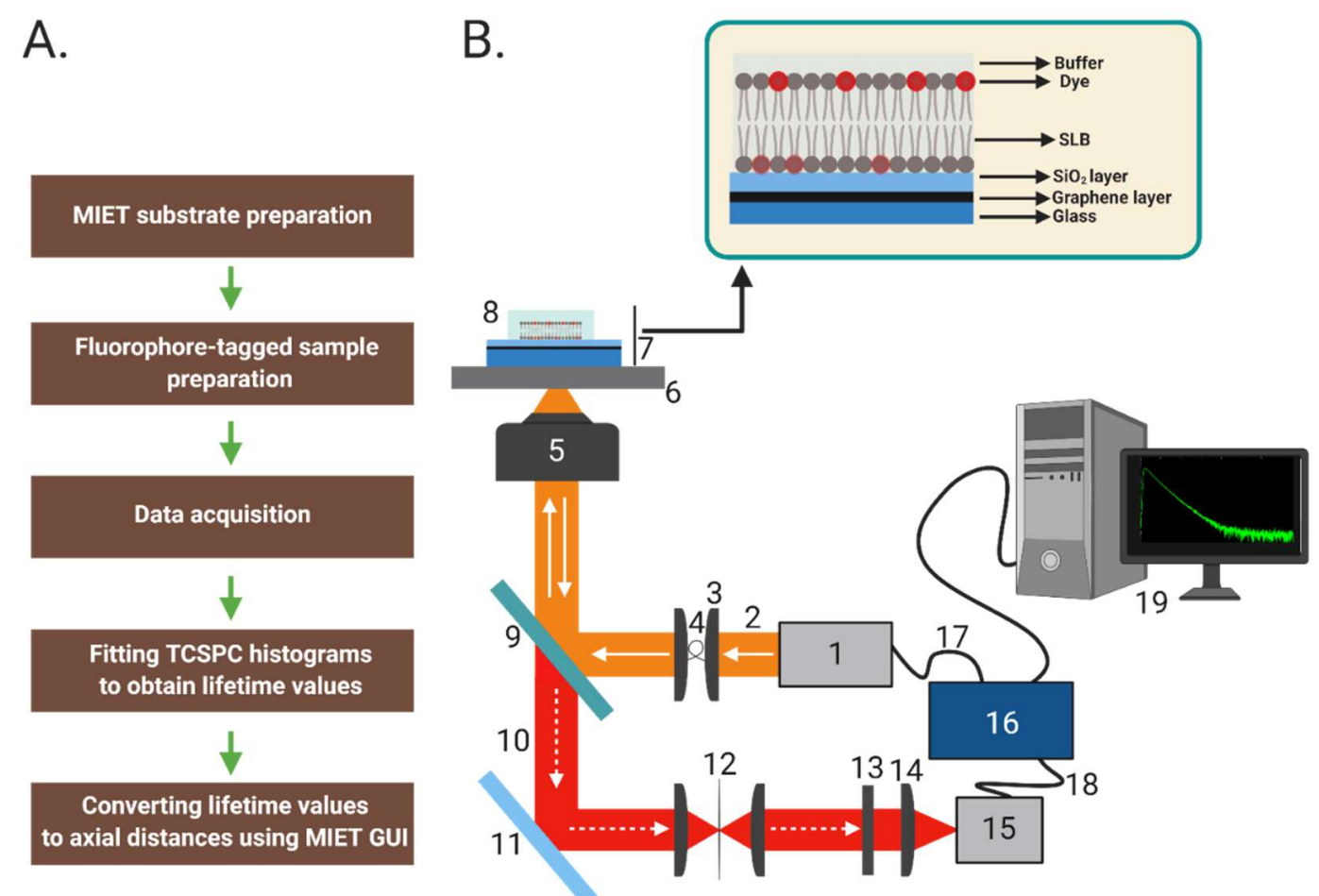


Figure 3: A. Workflow of MIET from substrate preparation to data analysis. B. FLIM setup used for MIET. 1. pulsed excitation laser source 2. excitation beam (solid arrow showing propagation direction) 3. collimating lens 4. optical fibre for coupling 5. objective lens 6. scanning stage 7. GIET substrate 8. sample - here supported lipid bilayer (SLB) on GIET substrate. 9. dichroic mirror 10. emission beam (white dashed arrow showing propagation direction) 11. mirror 12. pinhole 13. band pass filter 14. lens 15. single photon counting module 16. timing electronics 17. laser sync 18. detector sync. 19. computer. The inset shows a magnified view of the sample with the GIET substrate.

Throughout this work, we use $\lambda_{\mathrm{exc}}=645 \mathrm{~nm}$. The excitation beam is reflected by a dichroic mirror (Di01-R405/488/561/635, Semrock) towards the objective. Back-scattered excitation light is blocked by a long-pass filter (BLP01-635R, Semrock). Collected fluorescence is focused through a pinhole of $100 \mu \mathrm{m}$ diameter for rejecting out-of-focus light. After the pinhole, the fluorescence light is recollimated and then refocused onto the active area of a single-photon avalanche photo diode after passing through a suitable band-pass filter (692/40 BrightLine ${ }^{\circledR}$ single-band bandpass filter, Semrock). For measurements with living cells under physiological conditions, the microscope is equipped with an incubator (LIS, CB02A).

\section{Substrate preparation}

For MIET measurements on living cells, glass-bottom petri-dishes (ibidi $\mathrm{GmbH}$ ) were coated with the following multilayer structure: $2 \mathrm{~nm}$ Ti below, then $20 \mathrm{~nm}$ Au and $1 \mathrm{~nm}$ Ti on top. The metal films were prepared by vapour deposition onto a cleaned glass cover slide (thickness $170 \mu \mathrm{m}$ ) using an electron beam source (Univex 350; Leybold) under high-vacuum conditions $\left(\sim 10^{-6} \mathrm{mbar}\right)$. Film thickness was monitored using an oscillating quartz unit during vapour deposition, and afterwards verified by atomic force microscopy. For GIET, glass slides that were already coated with one monolayer of graphene were commercially purchased (Graphene Supermarket, New York, USA). For experiments, silica layers of various thicknesses, namely 10,15 and $20 \mathrm{~nm}$ were evaporated on top of the graphene using the same vapour deposition procedure.

\section{Sample preparation}

Single molecule measurements For single-molecule GIET measurements, we used silicon dioxide spacers of three different thickness values: 10, 15 and $20 \mathrm{~nm}$. As mentioned above, these spacers were evaporated directly on the graphene coated glass coverslips. For single-molecule FLIM, Atto655 (ATTO-TEC GmbH, Siegen, Germany) molecules were spin-coated on top of these substrate. This dye was chosen for its well-known high photostability in air. Briefly, $1 \mathrm{nM}$ stock solution of Atto655 
was diluted to $100 \mathrm{pM}$ in Millipore water $\left(18.2 \mathrm{M} \Omega \mathrm{cm}\right.$ at $\left.25^{\circ} \mathrm{C}\right)$, and a $10 \mu \mathrm{l}$ aliquot of this solution was spin-coated onto substrates at $8000 \mathrm{rpm}$ for $50 \mathrm{~s}$ (see Figure 4A).

Supported lipid bilayer (SLB) preparation. Supported lipid bilayers (SLBs) were prepared by vesicle fusion to a substrate as described in [33]. 1,2-dioleoyl-sn-glycero-3-phosphocholine (DOPC) and 1,2dilauroyl-sn-glycero-3-phosphocholine (DLPC) lipids were purchased from Avanti Polar Lipids, Alabama, USA. Lipids with Atto655-tagged head groups, namely 1,2-dipalmitoyl-sn-glycero-3phosphoethanolamine (DPPE) and 1,2-dilauroyl-sn-glycero-3 phospho-ethanolamine (DLPE) were purchased from ATTO-TEC GmbH, Siegen, Germany. Unlabeled lipids were dissolved in chloroform at a concentration of $10 \mathrm{mg} / \mathrm{ml}$, whereas fluorescently labeled lipids were diluted to $0.01 \mathrm{mg} / \mathrm{ml}$. For preparation of Atto655-DLPE-DLPC bilayers, $1 \mu 1$ of Atto655-DLPE was mixed with $80 \mu$ of DLPC. The solution was dried in vacuum at $30^{\circ} \mathrm{C}$ for $1 \mathrm{~h}$ to evaporate the chloroform and for obtaining a thin film. This film was re-suspended with buffer solution $\left(20 \mathrm{mM}\right.$ Tris- $\mathrm{Cl}, 100 \mathrm{mM} \mathrm{NaCl}, 10 \mathrm{mM} \mathrm{CaCl}_{2}$, $\mathrm{pH}$ 7.4), followed by thermos-mixing (Thermomixer Comfort, Eppendorf) for $1 \mathrm{~h}$ at $30^{\circ} \mathrm{C}$. After mixing, the solution was passed through a polycarbonate membrane with pore diameter of $50 \mathrm{~nm}$ for extruding small unilamellar vesicles (SUVs). These SUVs were then deposited on the substrate and allowed to fuse for 1 hour to form a uniform bilayer. This incubation was followed by washing with buffer solution (20mM Tris-Cl, $100 \mathrm{mM} \mathrm{NaCl}, 10 \mathrm{mM} \mathrm{CaCl} 2, \mathrm{pH} 7.4)$ for at least 20 times to remove unbound vesicles.

Giant unilamellar vesicles (GUV) preparation. Giant unilamellar vesicles (GUVs) were prepared by electro-formation in a custom-built chamber as described elsewhere [34]. DOPC lipids and Atto655DPPE lipid labels were used to prepare GUVs [35]. A lipid mixture of $98 \mu 1$ of DOPC and $2 \mu 1$ of Atto655-DPPE in chloroform was deposited on the lower electrode plate and evaporated for $30 \mathrm{~min}$ under vacuum. The chamber was filled with $500 \mu \mathrm{l}$ of $300 \mathrm{mM}$ sucrose solution. Electro-formation was performed for $3 \mathrm{~h}$ at $15 \mathrm{~Hz}$ alternating current (AC) and a peak-to-peak voltage of $1.6 \mathrm{~V}$ followed by $8 \mathrm{~Hz}$ AC for $30 \mathrm{~min}$. $500 \mu \mathrm{l}$ of $300 \mathrm{mM}$ glucose was added to the chamber after electro-formation while simultaneously rinsing the electrode surface. The final suspension of GUVs was then collected from the chamber.

Cell membrane staining. MIET height profiling of the basal membrane of living cells was done for three different cell lines, MDA-MB-231, A549, and MDCK-II cells. MDA-MB-231 and A549 cells were cultured in Dulbecco's Modified Eagle Medium (DMEM, Lonza, Basel, Switzerland) with 4 mM L-Glutamine and 10\% FCS under standard conditions $\left(37^{\circ} \mathrm{C}\right.$ and $\left.5 \% \mathrm{CO}_{2}\right)$. MDCK-II cells were cultured using minimal essential medium (MEM, Biochrom, Berlin, Germany) with Earle's salts under 
the same conditions. For MIET experiments, approximately 20,000 MDA-MB-231 cells were seeded into a MIET substrate. Measurements were done within two days after seeding. A549 and MDCKII cells were seeded with the same density on gold-coated glass bottom petri dishes (MatTek, Ashland, MA, USA). Before measurements, the plasma membrane of cells was stained by incubating them with HEPES-buffered cell culture medium containing $5 \mu \mathrm{g} / \mathrm{ml}$ Cell Mask ${ }^{\mathrm{TM}}$ Deep Red Plasma Membrane Stain (Life Technologies, Darmstadt, Germany) for 5 minutes at $37^{\circ} \mathrm{C}$. The staining medium was replaced by HEPES-buffered cell medium during MIET measurements, which allowed for imaging living cells up to one hour after staining at $37^{\circ} \mathrm{C}$. In order to investigate spreading of MDCK-II cells, they were released from a culture flask by trypsinization for 5 minutes (trypsin/EDTA $0.5 \% / 0.2 \%$, Biochrom) and kept in suspension at a density of $10^{6}$ cells $/ \mathrm{ml}$. The cells were used for up to 1 hour. Gold-coated glass bottom petri dishes were incubated for 30 minutes with HEPES buffered cell medium for adhesion experiments. Cell medium was replaced by $400 \mu \mathrm{l}$ of staining solution (cell culture medium containing $5 \mu \mathrm{g} / \mathrm{ml}$ Cell Mask ${ }^{\mathrm{TM}}$ Deep Red Plasma Membrane Stain) and 50.000 cells were added. After 5 minutes at $37^{\circ} \mathrm{C}$, the staining solution was carefully replaced by HEPES-buffered cell culture medium, after which MIET experiments were started.

\section{Measurements and data analysis}

Single molecule localization. FLIM on surface-immobilized Atto655 molecules was done by scanning surface areas of $\sim 40 \mu \mathrm{m} \times 40 \mu \mathrm{m}$ with a pixel dwell time of $4 \mathrm{~ms}$ (100 nm effective pixel-size) with the aid of a three-axis piezo-nanopositioning stage (P-562.3CD, Physik Instrumente) and a dedicated piezo driver (E-710 Physik Instrumente). Fig. 4A illustrates the experimental design. A TCSPC histogram of photon arrival times with respect to excitation laser pulses was computed for each molecule by collecting photons from all pixels associated with one identified molecule. Next, a monoexponential decay function was fitted to the tail part of the decay histogram (cutoff $0.5 \mathrm{~ns}$ after maximum). Note that the cutoff value has to be larger than the width of the system's instrumental response function (IRF). Fluorescence lifetime fitting can be done by either of the two standard ways, (i) fitting the tail of the TCSPC histogram with a cutoff of usually 0.1-0.5 ns after the maximum of the histogram or (ii) full deconvolution using an IRF which can be separately recorded or extracted from background-only signal as described previously [36, 37]. 
A.

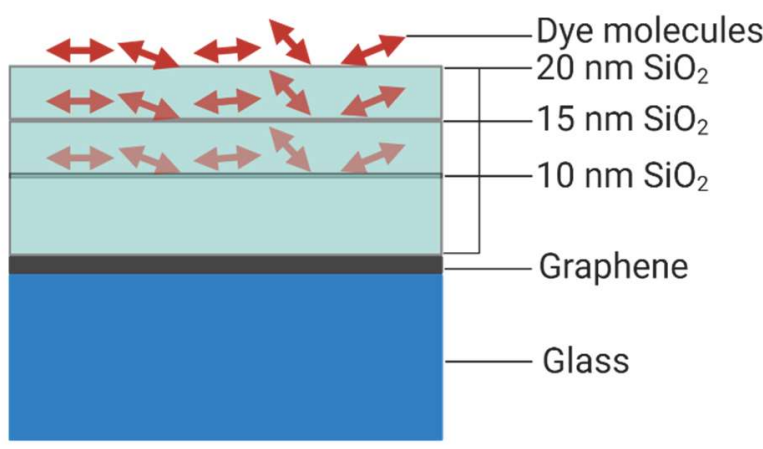

C.

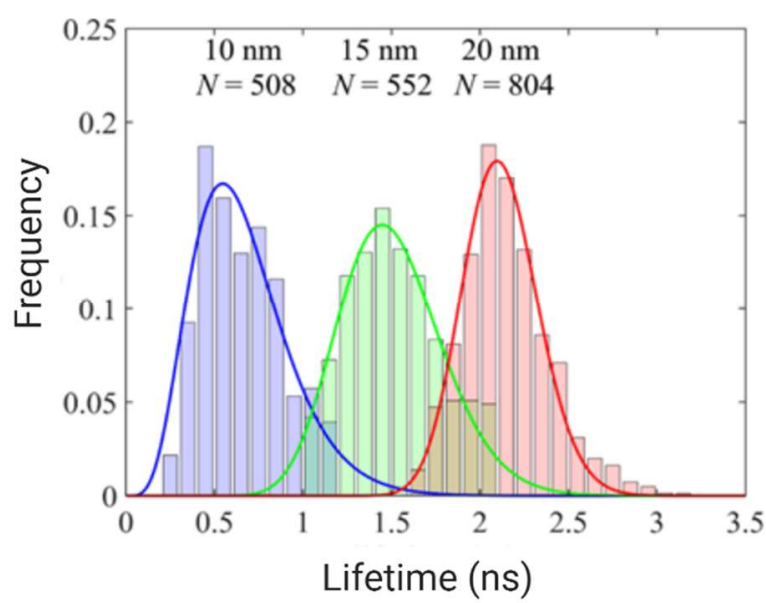

B.

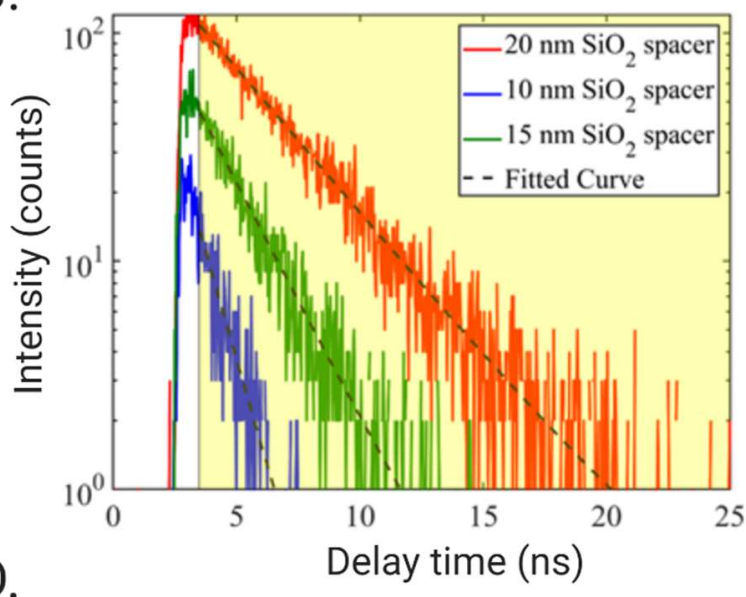

D.

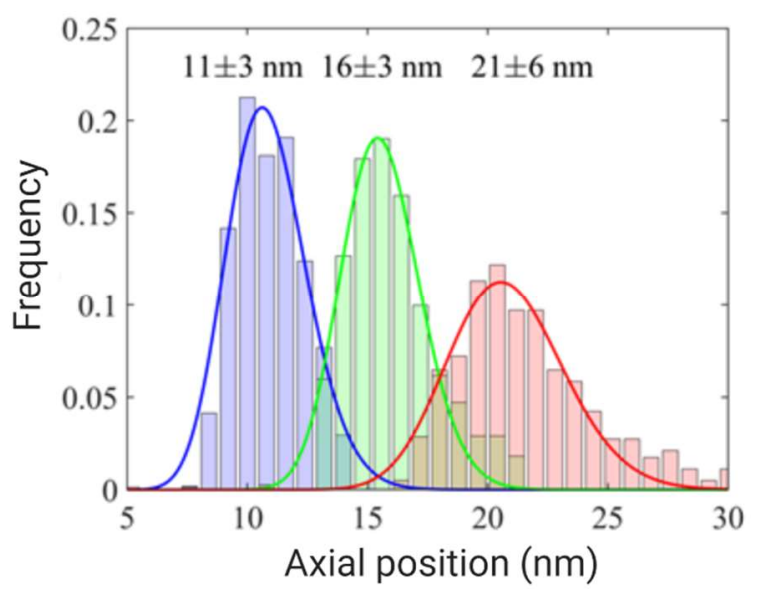

Figure 4: Single-molecule localization by GIET. A. Experimental design showing Atto655 molecules spin-coated on a GIET substrate with 10, 15 or $20 \mathrm{~nm}$ of $\mathrm{SiO}_{2}$ spacer layer evaporated on top of the graphene. B. Example fluorescence decay curves for three different molecules on the three studied substrates. The yellow shaded region is used for a maximum likelihood monoexponential tail fit of the fluorescence decay time. C. Lifetime distributions as obtained by tailfitting the decay curves of individual molecules. Numbers above the distributions indicate how many molecules contributed to the corresponding distribution. D. Distance distributions as obtained by converting the lifetime values from $C$. Solid lines represent fits of a general gamma distribution to the histograms. Data and figures B, C and D were adapted from [16].

Fig. 4B shows three representative TCSPC histograms and their corresponding fits, each for one molecule spin-coated on a silica spacer of thickness 10, 15 and $20 \mathrm{~nm}$, respectively. Lifetime 
distributions obtained from tail-fitting single molecule decay curves are shown in 4C where the number $N$ of individual molecules contributing to each distribution are also given. The free-space lifetime value was measured to be $\tau_{0}=2.9 \mathrm{~ns}$, while the quantum yield of the molecule, measured with the nanocavity method, was found to be $\varphi=0.25$. The orientation distribution of the fluorophores on the substrates was determined in a separate experiment using defocused imaging. Using this orientation distribution, an orientation-averaged GIET calibration curve was computed which was then used to convert lifetime values (Fig. 4C) to axial distance values (Fig. 4D). In the presented measurements, the obtained height values were $11 \pm 3,16 \pm 3$ and $21 \pm 6 \mathrm{~nm}$ for the silica spacers of 10,15 and 20 $\mathrm{nm}$ thickness, respectively. A systematic bias of $1 \mathrm{~nm}$ towards larger distance values could be due to surface roughness or to surface-induced heterogeneity of the photophysical parameters (free-space lifetime and QY) of the fluorophores. Nevertheless, it should be emphasized that the relative differences of vertical positions are reproduced correctly, showing a $5 \mathrm{~nm}$ increase from sample to sample.

Experiments on model membranes. GIET measurement were performed on SLBs composed of Atto655-tagged lipid head groups. The goal of the measurement was to determine the thickness inter of these model membranes (or more correctly, the distance between fluorescent dyes on different side of a bilayer). Fluorescence decay curves were recorded on SLBs at $20^{\circ} \mathrm{C}$ by scanning areas of $\sim 5 \mathrm{~m}$ $\times 5 \mu \mathrm{m}$. Recorded TCSPC histograms were de-convolved with the a priori measured instrument response function (IRF) (see Figure 5A) and then fitted with a bi-exponential decay function. The used MATLAB software package for lifetime fitting is freely available at https:/www.joergenderlein.de/software/Fluorescence lifetime fitting. The right panel of Fig. 5A presents the obtained fluorescence decay time distributions as a function of the collected number $N$ of photons per TCSPC for a DLPC bilayer. The orientation of Atto655 molecules attached to lipid head groups was determined separately by fluorescence polarization imaging of giant unilamellar vesicles (GUVs) using the same lipid molecules. These measurements were done on a home-built wide-field microscope. A linearly polarized laser beam $\left(\lambda_{\text {exc }}=637 \mathrm{~nm}\right.$, OEM-SD-637-500) was used for excitation of the labelled GUVs, and fluorescence was imaged with an emCCD camera (Ixon Ultra 897, Andor Technology, Ireland). The seen intensity distribution around the circumference of a GUV is shown in Fig. 5B. The intensity maximum at angles orthogonal to the excitation polarization indicates that dyes are oriented tangentially to the bilayer's surface. The free-space lifetime and QY of lipid-bound Atto655 were measured to be $\tau_{0}=2.6 \mathrm{~ns}$ and $\mathrm{QY}=0.36$, respectively. We used these values to compute 
the GIET calibration curve for a lipid membranes as shown in Fig. 5C (left panel). The model calculation takes into account the presence of the SLB, assuming that it has a refractive index of 1.46

A.

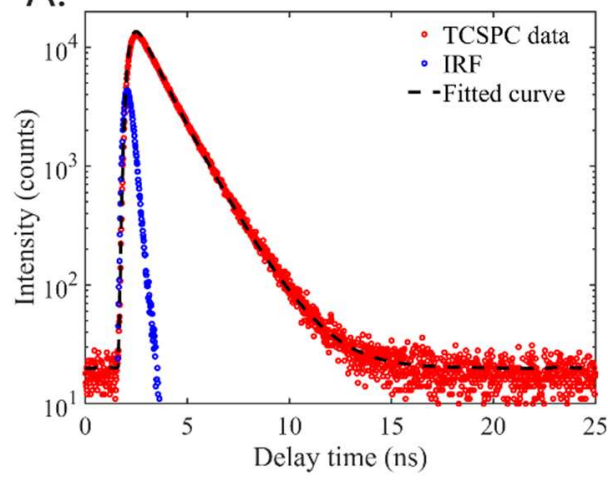

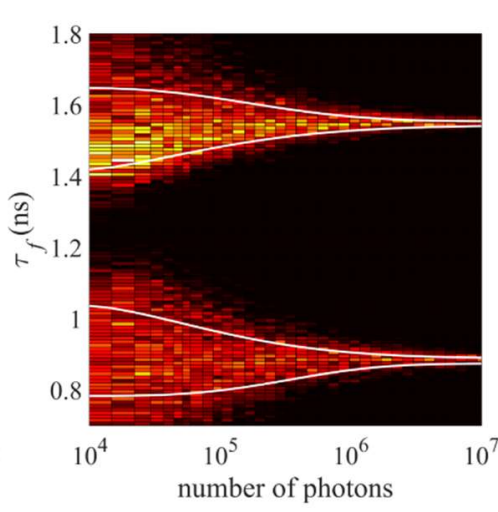

B.

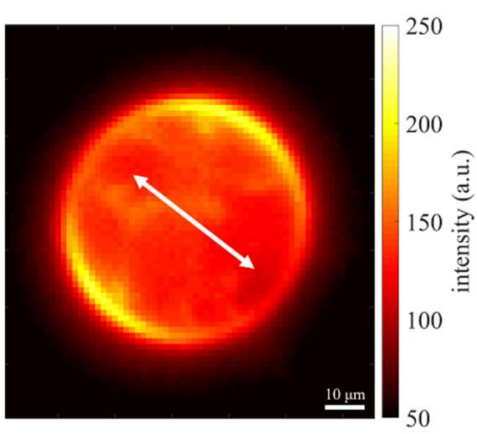

C.
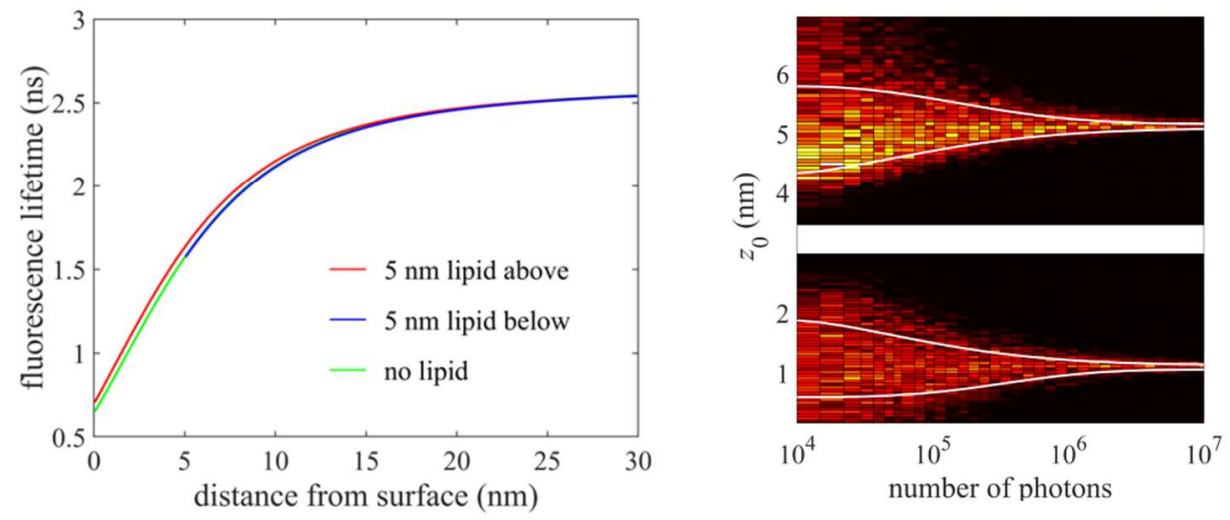

Figure 5: GIET experiments on supported lipid bilayer. A. Left panel demonstrates TCSPC decay and lifetime fit from DLPC SLB. The measured decay curve is red, instrument response function (IRF) is blue, and fitted curve is shown by a black dashed line. Right panel shows lifetime distributions for DLPC as a function of photons per TCSPC curve. The solid white lines show the mean value \pm the square root of the variance of the lifetime distributions as a function of photon number. B. Image of a GUV under linearly polarized excitation (the double-headed arrow indicates the excitation polarization). C. Left panel show MIET curves for the bilayer experiment. To estimate the impact of a $\sim 5 \mathrm{~nm}$ thick bilayer of refractive index 1.46, we calculated MIET curves assuming no bilayer was present (green), with a bilayer above (red) and with a bilayer below (blue) the fluorophore's position. Right panel depicts distance distribution corresponding to lifetime distributions as in A (right panel). For converting lifetime to distance, we used the blue curve in left panel for all lifetime values larger than $1.25 \mathrm{~ns}$ and the red curve for all lifetime values smaller than $1.25 \mathrm{~ns}$. The white gap reflects the difference between the red and blue curve. Data and figures $A$ (right panel), B and $C$ were adapted from [16]. 
and an approximate bilayer thickness of $5 \mathrm{~nm}$. For converting lifetime into distance values as illustrated in Fig. 5C (right panel) for DLPC SLB, we used the model curve labeled ' $5 \mathrm{~nm}$ lipid above' for the short lifetime component and the one labeled ' $5 \mathrm{~nm}$ lipid below' for the long lifetime component. As can be seen in Fig. 5C, the relative width of the distributions become narrower with increasing number $N$ of photons per TCSPC curve. In the limit large $N\left(\sim 10^{7}\right.$ photons $)$, we determined the position of the fluorophores with sub-nanometre accuracy. For DLPC SLB we obtain axial distance values for the bottom and top leaflets of $1.09 \pm 0.04 \mathrm{~nm}$ and $5.13 \pm 0.04 \mathrm{~nm}$, respectively, giving a bilayer thickness value of $4.04 \pm 0.06 \mathrm{~nm}$. Similarly, for DOPC SLB, we obtained dye positions of $1.4 \pm 0.1 \mathrm{~nm}$ and 6.9 $\pm 0.1 \mathrm{~nm}$ for the bottom and top leaflet, respectively, yielding a bilayer thickness value of $5.5 \pm 0.2$ $\mathrm{nm}$. Of course, these values refer to a bilayer thickness plus linker and dye. We measured also the height of the bottom leaflet above the silica surface, providing an estimate of the hydration layer between substrate and membrane. Our findings are in excellent agreement with previously reported values using SAXS and AFM [38, 39, 40]. The results presented and discussed in the current and previous sections have been adapted from Ref. [16].

Live cell nanoscopy. MIET imaging was used for time-lapse mapping of the basal membranes of living cells. We used MIET also for monitoring the spreading of adherent MDCK II cells, where we could monitor three different phases of interaction between cell membrane and surface. The first phase is dominated by the formation of initial bonds between adhesion molecules and the extracellular matrix (ECM). The initial tethering of a cell membrane is followed by a second phase that is mainly characterized by initial cell spreading driven by actin polymerization that forces a cell's surface area to increase by drawing membrane molecule from a reservoir of folded membrane regions and blebs. A third and final phase is governed by recruitment of additional plasma membrane from internally stored membrane buffers, and is characterized by extension of lamellipodia which leads to the occupation of a larger area by a cell. Experiments were done by recording time-lapse series of FLIM images with time interval of 5 minutes. Cells were scanned with a focused laser using a piezo-nanopositioning stage (P-562.3CD, Physik Instrumente $\mathrm{GmbH})$. Fluorescence photons were recorded in time-tagged, time-resolved mode, which allows for gathering all photons from a single pixel and sorting them into a histogram according to their arrival time after the last laser pulse. These TCSPC histograms were corrected for detector and electronics dead-time effects [41], and finally fitted with a multi-exponential decay function (fit of tail starting $0.1 \mathrm{~ns}$ after decay maximum) in each pixel. Conversion of lifetime values to axial distances and three-dimensional reconstruction of the membrane 
profile from the recorded FLIM images were done using a MATLAB-based GUI [21] which was developed for evaluating MIET measurements and which can be freely obtained at https://projects.gwdg.de/projects/miet. Fig. 6A illustrates the start window of this MIET GUI

A.

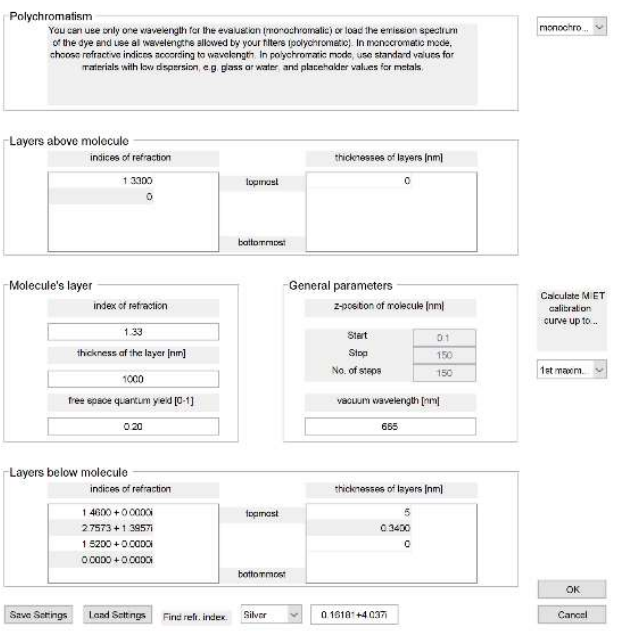

C.

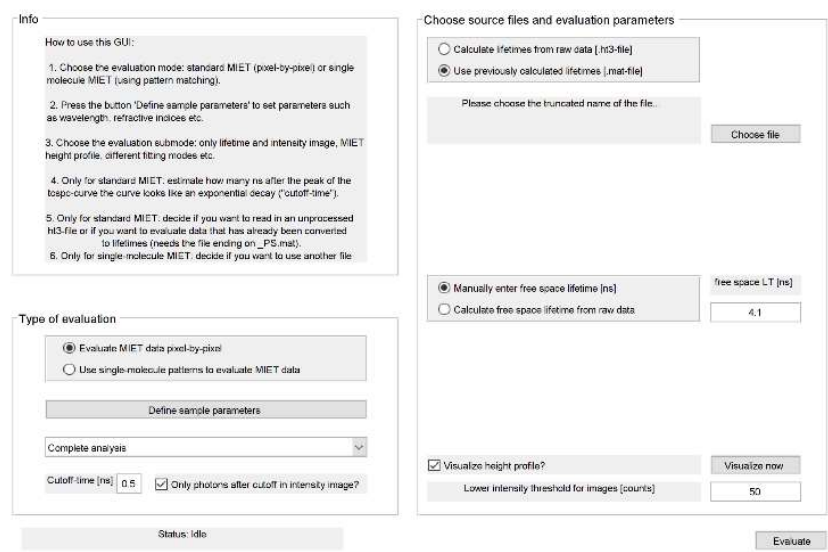

B.

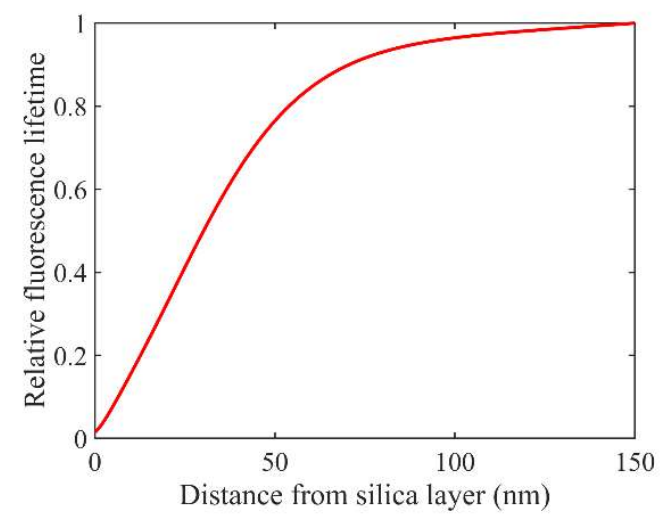

D.
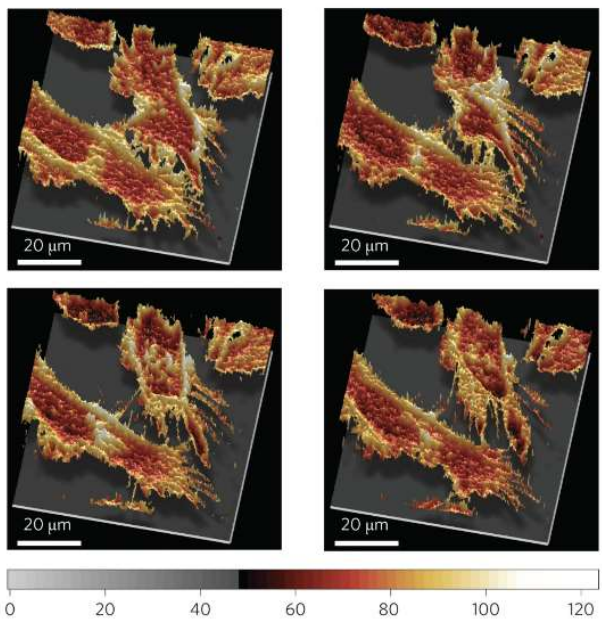

Figure 6: MIET GUI. A. Screenshot of the user interface of the MIET GUI which allows for supply values of thickness and refractive index of MIET substrate layers, and of the free-space lifetime and quantum yield of the used dye. B. Exemplary MIET curve as calculated by the MIET GUI. C. Screenshot showing GUI for loading lifetime values and fitting lifetimes from raw data as well as visualization options for displaying height profiles. D. Three-dimensional reconstruction of height images from lifetime images for the basal membrane of stained MDAMB-231 cells. Four images from a time-lapse series are shown. Data adapted and reprinted with permission from Ref. [15]. 
interface where one enters the different thickness and refractive index values for a MIET substrate layers, and the free-space lifetime and quantum yield of the used fluorophore. The app then calculates the MIET calibration curve (Fig. 6B) which is then used for the lifetime-to-distance conversion. In a second window of the GUI (Fig. 6C), the user then loads either a .mat file with already fitted fluorescence lifetime values, or recorded raw FLIM data (.ht3 format). In the latter case, lifetime fitting is done by the software itself. Next, the user can check the box of 'Visualize height profile'. Additionally, one can indicate the minimum threshold number of photons to be considered for lifetime fitting/conversion of lifetime values to axial distances. This allows for rejecting background and facilitates accurate filtering of FLIM images. Three-dimensional height profiles computed from fluorescence lifetime images that were recorded over 40 min on MDA-MB-231 cells are shown in Fig. 6D. We compared the mean membrane-metal surface distances of three different cell types and found that the benign epithelial cell line MDCK II is much closer to the surface with a height of $28 \pm 5 \mathrm{~nm}$ as compared to $54 \pm 8$ and $67 \pm 7 \mathrm{~nm}$ for MDA-MB-231 and A549 cell lines, respectively (Fig. 7). 

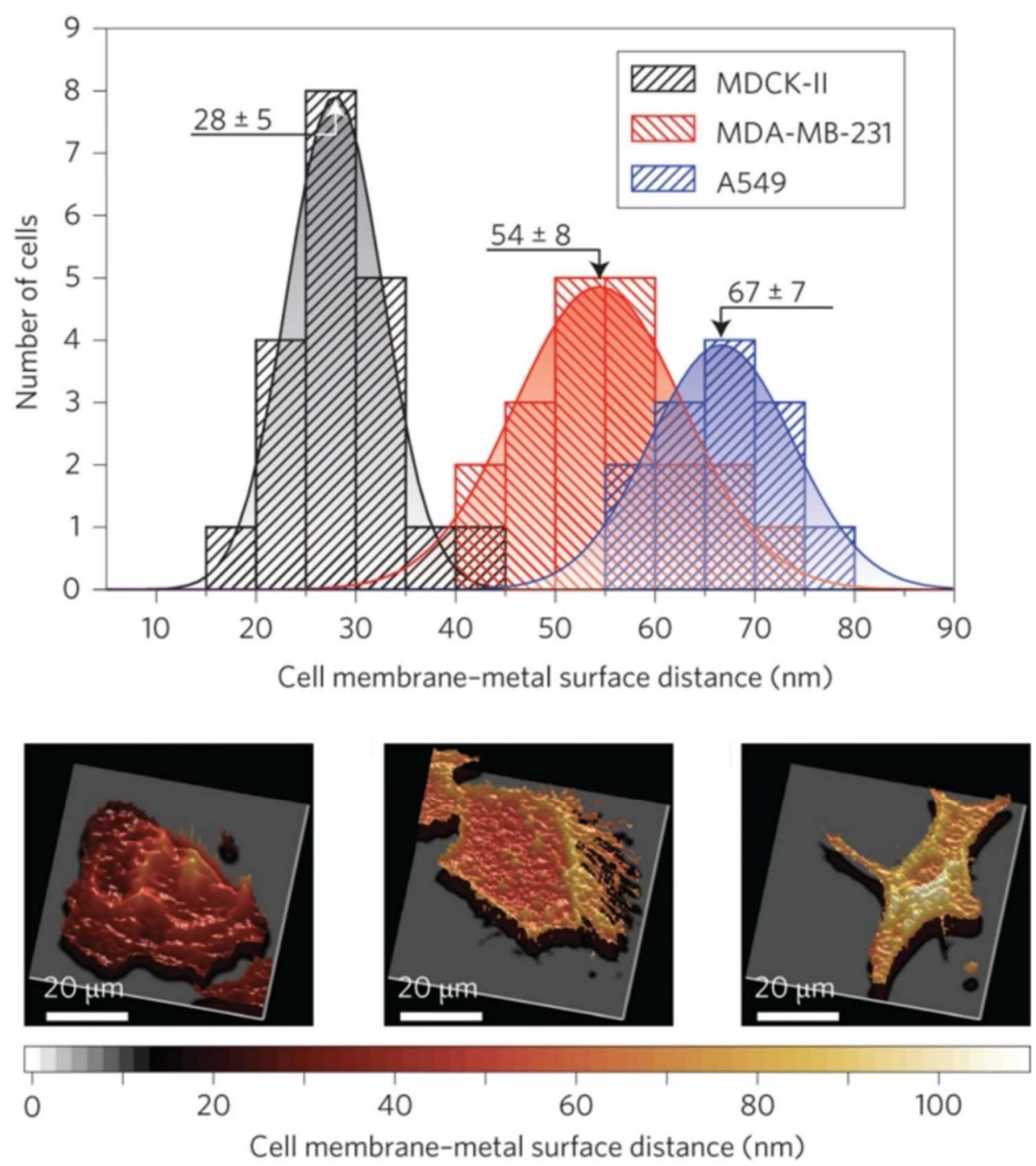

Figure 7: Comparison of basal membrane profiles of three different cell types. Top panel: Average distance of basal membrane from gold surface for MDA-MB-231, A549 and MDCK-II cell lines. Solid curves show Gaussian function fits to the experimental data. Bottom panel: Three-dimensional reconstructions of the basal membrane of a MDCK-II (left), an MDA-MB231 (centre) and a A549 (right) cell. For better comparison, all images are normalized to the same height scale. Figures adapted with permission from Ref. [15]. 


\section{MATERIALS}

\section{REAGENTS}

- Atto655-NHS (ATTO-TEC GmbH)

- 1,2-dioleoyl-sn-glycero-3-phosphocholine (DOPC); 1,2-dilauroyl-sn-glycero-3-phosphocholine (DLPC) (Avanti Polar Lipids)

- Atto655 tagged 1,2-dipalmitoyl-sn-glycero-3-phosphoethanolamine (DPPE); 1,2-

Dilauroyl-sn-glycero-3-phosphoethanolamine (DLPE) (ATTO-TEC GmbH)

- Chloroform (Sigma Aldrich)

- $\quad$ Tris-Cl buffer (20mM Tris-Cl, $\left.100 \mathrm{mM} \mathrm{NaCl}, 10 \mathrm{mM} \mathrm{CaCl}_{2}, \mathrm{pH} 7.4\right)$

- Glucose (Sigma Aldrich)

- Cell lines: MDA-MB-231, A549 and MDCK II

- Dulbecco's Modified Eagle Medium (DMEM, Lonza)

- Minimal Essential Medium (MEM, Biochrom)

- L-Glutamine (Sigma Aldrich)

- Fetal calf serum (Sigma Aldrich)

- Cell Mask ${ }^{\mathrm{TM}}$ Deep Red Plasma Membrane Stain (Life Technologies).

- Glass bottom petri dishes (MatTek)

- Trypsin/EDTA $0.5 \% / 0.2 \%$ (Biochrom)

\section{EQUIPMENT}

- Optical table, vibration isolated (Newport)

- Inverted fluorescence microscope IX 71 (Olympus)

- High numerical aperture objective lens (eg. 100× UPLSAPO, N.A. 1.4 (Olympus)or 1.49 NA)

- Objective for alignment (10×; Carl Zeiss, Olympus)

- Pulsed diode laser ( $\lambda_{\text {exc }}=640$ nm, LDH-D-C 640, PicoQuant GmbH)

- $\quad$ Three-axis piezo stage (P-562.3CD, Physik Instrumente)

- Lenses (Thorlabs)

- Laser clean-up filter 640/8 nm MaxDiode ${ }^{\mathrm{TM}}$ laser clean-up filter (Semrock).

- Neutral density filter to adjust laser intensity (Edmund Optics).

- 405/488/561/635 nm lasers BrightLine ${ }^{\circledR}$ quad-edge laser dichroic beamsplitter (Semrock). 
- Long-pass filter BLP01-635R (Semrock)

- Emission filter: 692/40 nm BrightLine ${ }^{\circledR}$ single-band bandpass filter (Semrock). Recommended for experiments using a gold layer.

- Detector: Single photon counting module SPCM-AQRH (Excelitas Technologies).

- Fiber coupler 60SMS-1-4-RGB11-47 (Schäfter and Kirchhoff) and polarisation-maintaining single-mode optical fiber (PMC-400-4.2-NA010-3-APC-250 V, (Schäfter and Kirchhoff).

- Multichannel picosecond diode laser driver: PDL 828 "Sepia II" (PicoQuant GmbH).

- Multichannel picosecond event timer \& TCSPC module: HydraHarp 400 (PicoQuant GmbH).

- System control devices: Computer (Windows 10 x64, Intel Core i5-4590, 16 GB RAM)

Software

- Relevant driver and application for each device listed in EQUIPMENT.

- Fluorescence lifetime imaging and correlation software: Symphotime 64 (PicoQuant GmbH).

\section{REAGENT SETUP}

Sample for calibration and drift control In order to verify the alignment of the microscope and single molecule detection with high SNR (signal to noise ratio), Atto655 molecules are spin coated on cleaned glass coverslips at single molecule concentration and FLIM images were recorded.

Cell culture In principle, any cell types are compatible for MIET experiments, but adherent cell lines offer additional advantage of minimal sample movement. Cells are grown) with $4 \mathrm{mM}$ L-Glutamine and $10 \%$ FCS under standard conditions $\left(37^{\circ} \mathrm{C}\right.$ and $\left.5 \% \mathrm{CO}_{2}\right)$.

Supported lipid bilayer For the preparation of SLB samples with adequate concentration of diffusing fluorophores in and out of the focus, a ratio of 1:80000 is maintained between fluorescently labeled and unlabeled lipids. For verification and quality check of the membrane prepared, it is preferred to do a FCS (fluorescence correlation spectroscopy) or a FRAP (fluorescence recovery after photobleaching) experiment before the actual measurement. Diffusion coefficient of lipid molecules as obtained from FCS is then compared with the previously reported values to ensure SLB formation. On the other hand, FRAP enables to detect whether the sample is composed of non-fused SUVs or a uniform membrane has formed. 


\section{PROCEDURE}

\section{FLIM microscope setup $\bullet T I M I N G 1 \mathrm{~h}$}

i. A properly aligned FLIM microscope with single molecule detection efficiency with high SNR is an essential prerequisite of $(\mathrm{g})$ MIET experiments.

ii. Laser intensity before and after the fibre coupler has to be monitored with a power meter to check the coupling efficiency and should be adjusted accordingly to reach the maximum coupling efficiency.

iii. Following this, laser power in the back focal plane of the objective lens should be measured to keep a track of effective laser power in the experiments.

iv. In the emission path, detector and the pinhole should be perfectly aligned to ensure maximum detection efficiency. ? TROUBLESHOOTING

v. Calibration measurement with a reference sample, as mentioned before is essential prior to the actual measurements.

\section{Preparation of single-molecule sample • TIMING $15 \mathrm{~m}$}

i. $\quad$ Prepare a dye solution (e.g. Atto655) of $100 \mathrm{pM}$ concentration by serial dilution from stock.

ii. Spin coat $10 \mu \mathrm{l}$ of the same solution on cleaned glass coverslip @8000 rpm for 40 seconds.

\section{Preparation of supported lipid bilayer $\bullet T I M I N G$ G-5 $\mathrm{h}$}

i. Prepare stock solution of unlabelled lipids at a concentration of $10 \mathrm{mg} / \mathrm{ml}$ and for labeled lipids at $0.01 \mathrm{mg} / \mathrm{ml}$. Mix $1 \mu \mathrm{l}$ of labelled lipid to $80 \mu 1$ of unlabelled lipid.

ii. Dry the mixture in vacuum at $30^{\circ} \mathrm{C}$ for 1 hour to get rid of any residual solvent and obtain a thin film. CRITICAL it is essential to ensure proper drying and get rid of any residual solvent for the formation of the desired thin film.

iii. Re-suspend the film with buffer solution (20mM Tris-Cl, $100 \mathrm{mM} \mathrm{NaCl}, 10 \mathrm{mM} \mathrm{CaCl}_{2}, \mathrm{pH}$ 7.4).

iv. Thermo-mix the re-suspended solution (Thermomixer Comfort, Eppendorf) for $1 \mathrm{~h}$ at $30^{\circ}$ C.

v. Extrude the SUVs from this solution by passing the same through a polycarbonate membrane with a pore diameter of $50 \mathrm{~nm}$.

vi. Deposit the SUVs on the substrate and allow it to fuse for 1 hour @ RT for the formation of a uniform lipid bilayer. 
vii. Wash with buffer solution multiple times following incubation for removing unbound vesicles. ? TROUBLESHOOTING

\section{Preparation of cell samples • TIMING $3 \mathrm{~d}$}

i. Culture MDA-MB-231 and A549 cells in Dulbecco's Modified Eagle Medium (DMEM, Lonza) with $4 \mathrm{mM}$ L-Glutamine and $10 \%$ FCS under standard conditions $\left(37^{\circ} \mathrm{C}\right.$ and $5 \%$ $\mathrm{CO}_{2}$ ).

ii. MDCK-II cells were cultured under the same conditions except for using minimal essential medium (MEM, Biochrom) with Earle's salts instead of DMEM.

iii. Seed 20000 cells of each cell type in a reservoir of diameter $1 \mathrm{~cm}$ on gold coated glass bottom petri dishes.

iv. Experiments should be done within two days of seeding.

v. Prior to MIET measurements, stain the plasma membrane of the cells by incubating living cells with HEPES-buffered cell culture medium containing $5 \mu \mathrm{g} / \mathrm{ml}$ Cell Mask ${ }^{\mathrm{TM}}$ Deep Red Plasma Membrane Stain (Life Technologies) for 5 minutes at $37^{\circ} \mathrm{C}$.

vi. For the actual measurement, replace the staining medium by HEPES-buffered cell medium, to allow the investigation of living cells up to one hour after staining at $37^{\circ} \mathrm{C}$.

\section{FLIM imaging of single molecules $\bullet T I M I N G 1 \mathrm{~h}$}

i. Set the scanning parameters in the SymPhoTime 'Measurement' mode. In particular, specify the area to be scanned on the sample for e.g. $40 \times 40 \mu \mathrm{m}^{2}$; effective virtual pixel size for e.g. $100 \mathrm{~nm}$; dwell time per pixel depending on photostability of the fluorophore used for e.g. $2 \mathrm{~ms} /$ pixel;

ii. Do a quick test area scan to check the background signal originating from MIET substrate or other sources. ? TROUBLESHOOTING

iii. Record FLIM images of immobilized dye molecules on glass for estimating the free-space lifetime.

iv. Continue with the area scans on MIET substrate with low laser power until the single molecules bleach.

\section{Measurements on SLB • TIMING $2 \mathrm{~h}$}


i. Scan several representative areas on the SLB sample for e.g. $5 \times 5 \mu \mathrm{m}^{2}$ prepared on a glass coverslip for estimation of the free-space lifetime of dye-tagged lipid head-group.

ii. Before the actual GIET experiment, record the instrument response function (IRF), preferably with the same laser intensity as for the GIET measurements. IMPORTANT it is essential to record of IRF and lifetime measurements on bilayer with the same laser intensity or to cross check independently that the shape of the IRF doesn't vary with laser intensity.

iii. Record the TCSPC histogram until the number of photons collected in the highest bin reaches one million or more.

iv. Take care to keep the count rates as such to avoid detector dead time artefacts.

v. Do a quick comparison in the software's 'Analysis' window between the fluorescence lifetimes obtained from GIET and glass measurements. Often at times you can end up in preparing a multilayer or a bilayer with lot of unbound vesicles showing up as a longer lifetime than expected. ? TROUBLESHOOTING

\section{Measurements on live cells $\bullet T I M I N G 1 \mathrm{~h}$}

i. For measuring living cells under physiological conditions, the microscope was equipped with an incubator (LIS, CB02A).

ii. Scan individual cells with the focused laser beam with optimized scanning parameters in order to collect $\sim 1000$ photons on an average per pixel.

iii. Take care to adjust the excitation intensity and count rates as such to avoid dead time artefacts? TROUBLESHOOTING

\section{Single-molecule data evaluation $\bullet$ TIMING $30 \mathrm{~min}$}

i. Launch the 'MIET GUI' in MATLAB. Select 'Use single-molecule patterns to evaluate MIET'

ii. Click 'Define sample parameters' and provide the parameters in the new pop up window that appears enlisting refractive indices of layers above, below and molecule's layer, numerical aperture of the objective lens used, excitation and emission wavelengths of the fluorophore used (maximum value of spectra). Click 'OK'. 
iii. Open the dropdown menu in original window below 'Define sample parameters' section. Select 'Complete analysis of single molecules using pattern detection' from the dropdown options.

iv. On the right hand side of the GUI window, either choose 'Calculate lifetimes from raw data [.ht3 file]' or you can also provide previously fitted lifetime values a .mat file from your computer using 'Choose file' option.

v. If you wish to fit lifetimes by full deconvolution using an IRF, either click 'Estimate IRF' which estimates the IRF from background signal or click 'Calculate IRF from extra data' and upload a raw file [.ht3] where you recorded the IRF separately.

vi. Enter the free space lifetime value if known previously or click on 'Calculate free space lifetime from raw data' and select the raw file [.ht3] containing lifetime measurement on glass.

vii. Finally, click on 'Evaluate'.

viii. For single molecule scans, indicate the minimum number of photons collected per individual molecule to get rid of photobleached, dim molecules during the scans.

ix. We use a 'Gaussian mask' and pattern matching to identify the pixels with single molecules and then generate a background-filtered intensity image showing identified single molecules.

x. For each molecule, a TCSPC histogram was computed from the photons collected from the identified individual pixels.

xi. A mono-exponential decay function was either fitted to the tail of the histogram ( $0.5 \mathrm{~ns}$ after the maximum) or full deconvolution using IRF

xii. Plot lifetime and height distributions as obtained from the GUI analysis algorithm. (shown in figure 4). ? TROUBLESHOOTING

xiii. Alternatively, for a fixed dipole orientation of single molecules unlike 'random' conformation, the GUI requires an update which will be integrated soon. So, the example of single-molecule localization described in this article earlier uses a custom-written MATLAB routine for the analysis in order to take into account the orientation values. This can be easily provided to any interested user upon request until the upgradation of the GUI.

9. Data analysis for SLB measurements - TIMING $30 \mathrm{~m}$ (only if the programs are provided)

i. Compute the TCSPC histogram with the recorded photons. 
ii. We sectioned the total number of photons into $N$ number of bunches (why does one need this step?) and fitted the TCSPC curve of each bunch with a bi-exponential model function using a MATLAB based IRF deconvolution algorithm. ? TROUBLESHOOTING

iii. The MATLAB based software package for fluorescence lifetime fitting is available free of charge at https://www.joerg-enderlein.de/software.

iv. Lifetime distributions were generated from the fitted decay times as a function of number of photons per bunch per TCSPC curve as shown in Figure 5A.

v. Compute the MIET curve for SLBs. The model calculation takes into account the presence of the SLB, assuming a refractive index of 1.46 and membrane thickness of $5 \mathrm{~nm}$ using the MIET GUI.

vi. As shown in Figure 6, we use the curve ' $5 \mathrm{~nm}$ lipid above' for determining the position of bottom leaflet and ' $5 \mathrm{~nm}$ lipid below' for the top leaflet.

vii. We take the lifetime values at the limit of the photon numbers ( $10^{7}$ photons) and determine the axial positions using the MIET curve. (See Figure 5C).

\section{Three dimensional height image reconstruction using MIET GUI • TIMING $15 \mathrm{~m}$}

i. FLIM images of live cells were used to obtain the three dimensional reconstructed height image using the MATLAB based project MIET_GUI (available free of charge at https://projects.gwdg.de/projects/miet).

ii. Launch the 'MIET GUI' in MATLAB. Select 'Evaluate MIET data pixel-by-pixel'

iii. Click 'Define sample parameters' and provide the relevant parameters in the new pop up window as described previously such as the complex-valued refractive index of each layer and corresponding layer thicknesses, the quantum yield of the dye in corresponding slots. Click ' $\mathrm{OK}$ ' to go back to the main menu or the original GUI window.

iv. Open the dropdown menu in original window below 'Define sample parameters' section. Select 'Complete analysis' from the dropdown options.

v. Provide the 'Cutoff-time [ns] after the maximum of the lifetime histogram from where the tail of the histogram will be considered for lifetime fitting. Usually the value ranges from 0.1-0.5 ns depending on width of the IRF.

vi. Check the box saying 'Only photons after cutoff in intensity image?' 
vii. On the right hand side of the GUI window, either choose 'Calculate lifetimes from raw data [.ht3 file]' or you can also provide previously fitted lifetime values a .mat file from your computer using 'Choose file' option.

viii. Enter the free space lifetime value if known previously or click on 'Calculate free space lifetime from raw data' and select the raw file [.ht3] containing lifetime measurement on glass.

ix. Check the box saying 'Visualize height profile?' for visualizing height profile in 3D.

ix. Click 'Evaluate'. ? TROUBLESHOOTING

\section{TROUBLESHOOTING}

Step Problem

1

Laser beam not

propagating through

the centre of the

pinhole.

\section{Possible reason}

a. $x-y$ controllers

of the pinhole not

at correct position.

b. Beam height and centre.

$z$ position of pinhole not at par.

Beam not hitting

the centre of the

active area of the

detector. a. Proceed in a stepwise manner, place a pinhole with larger diameter for e.g. $200 \mu \mathrm{m}$, adjust the $x-y$ controller screws to bring the beam to b. Re-measure the beam height, adjust the $z$ position accordingly.

Place a low concentration $(\sim \mathrm{nM})$ of dye solution as sample. Focus the laser beam into the solution $(\sim 10 \mu \mathrm{m}$ from surface). Unscrew the pinhole. Adjust with the $x-y$ controllers of the lens in front of the detector to reach the point where the highest count rate, maximum brightness in FCS curve is observed.
Laser pulse sync not detected.
Connection of

Hydraharp 400 and

PC not working.
Switch off and restart the Hydraharp 400. Restart the software, if necessary restart the PC. Check if the laser driver is also on and working properly. 


\section{Longer fluorescence}

lifetime than

expected.

5

High background

from substrate.
Unbound vesicles present in the SLB sample.

High scattering from gold layer or uncleaned substrate.
Re-wash the sample with the buffer at least ten more times to remove remaining vesicles. Incubate the sample for 30 minutes again and restart measurement.

Use appropriate long-pass filter, shift to lower excitation intensity if possible.
5

Heterogeneous
distribution of
lifetime values.

5 Longer lifetime than expected.

\section{Contamination in} the sample from uncleaned substrate or dye solution contaminated.

\section{Re-wash the remaining slides from the same} batch in $\mathrm{KOH}$ and distilled water followed by plasma cleaning. Use a fresh dye solution diluted from a new stock.
Silica spacer's

thickness not accurate.
6
Lifetime values of
dyes on membrane
close to free-space
lifetime.
Formation of multilayers instead of bilayer.

Confirmation of the silica layer's thickness by AFM can be done.
Dead-time artefacts appear in TCSPC curve.
Higher count-rates than tolerated due to use of high

Discard the sample. Re-suspend a new batch of SUVs. Deposit the solution carefully on substrate followed by a longer incubation time ( $\sim 1$ hour) and repeated washes after incubation. 
excitation intensity dead-time correction of the data as described for densely labelled here [35].

sample.

8

Height values

deviating from

design.

9 recorded IRF.
Surface roughness

of the substrate.
Perform AFM measurements on the substrate to estimate root mean square value of the roughness. A rough surface can be avoided by skipping the plasma-cleaning step during washing the coverslips. Also, deposition of $\mathrm{SiO}_{2}$ layer should be done in slowest possible rate.
Misfit of the TCSPC

histogram with the

a. IRF shifted by few TCSPC bins compared to actual data.

b. Shape of the IRF different than the TCSPC curve due to different excitation intensity.
False positive height
High background in determination in some pixels. those pixels. a. Shift back the IRF during analysis by corresponding number of bins and refit using the modified IRF.

b. Record a new IRF using exactly the same settings for laser power as used for the measurement.

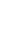


Step 8-10, Data evaluation, three-dimensional height reconstruction: 30 minutes -1 hour

\section{ANTICIPATED RESULTS}

a) Reference measurement

The reference measurement is a prerequisite before doing sample measurements for (g)MIET. Singlemolecule localization based experiments presented here serve as an ideal calibration experiment. It provides the proof of principle of distance dependent fluorescence lifetime modulation near a metal or graphene layer, at the same time it is a good check of single molecule detection sensitivity of the FLIM setup being used. Figure 4 illustrates single-molecule localization using GIET. A. Experimental design

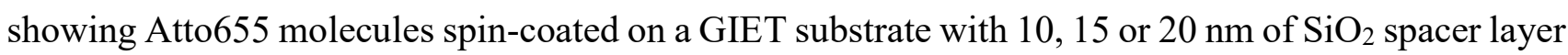
evaporated on top of the graphene. B. Example fluorescence decay curves for three different molecules on the three studied substrates. The yellow shaded region is used for a maximum likelihood monoexponential tail fit of the fluorescence decay time. C. Lifetime distributions as obtained by tail-fitting the decay curves of individual molecules. Numbers above the distributions indicate how many molecules contributed to the corresponding distribution. D. Distance distributions as obtained by converting the lifetime values from $\mathrm{C}$. Solid lines represent fits of a general gamma distribution to the histograms. Data and figures B, C and D were adapted from [16].

Figure 5 shows GIET experiments on supported lipid bilayer.

GIET experiments on supported lipid bilayer. A. Left panel demonstrates TCSPC decay and lifetime fit from DLPC SLB. The measured decay curve is red, instrument response function (IRF) is blue, and fitted curve is shown by a black dashed line. Right panel shows lifetime distributions for DLPC as a function of photons per TCSPC curve. The solid white lines show the mean value \pm the square root of the variance of the lifetime distributions as a function of photon number. B. Image of a GUV under linearly polarized excitation (the double-headed arrow indicates the excitation polarization). C. Left panel show MIET curves for the bilayer experiment. To estimate the impact of a $\sim 5 \mathrm{~nm}$ thick bilayer of refractive index 1.46, we calculated MIET curves assuming no bilayer was present (green), with a bilayer above (red) and with a bilayer below (blue) the fluorophore's position. Right panel depicts distance distribution corresponding to lifetime distributions as in A (right panel). For converting lifetime to distance, we used the blue curve in left panel for all lifetime values larger than $1.25 \mathrm{~ns}$ and the red curve for all lifetime values smaller than $1.25 \mathrm{~ns}$. The white gap reflects the difference between the red and blue curve. Data and figures A (right panel), B and C were adapted from [16]. 
Figure 7 depicts the comparison of basal membrane profiles of three different cell types. Top panel: Average distance of basal membrane from gold surface for MDA-MB-231, A549 and MDCK-II cell lines. Solid curves show Gaussian function fits to the experimental data. Bottom panel: Threedimensional reconstructions of the basal membrane of a MDCK-II (left), an MDA-MB-231 (centre) and a A549 (right) cell. For better comparison, all images are normalized to the same height scale. Figures adapted with permission from Ref. [15].

\section{Acknowledgements}

N.K. and A.G. are grateful to the Deutsche Forschungsgemeinschaft (DFG) for funding via project A06 of the Collaborative Research Centre SFB 860. J.E. acknowledges funding by the DFG under Germany’s Excellence Strategy - EXC 2067/1- 390729940.

\section{Author contributions}

J.E. conceived the project, helped in data analysis; A.G., A.I.C., and N.K. performed experiments and analysed the data; A.G. and J.E. wrote the manuscript with the input of all other authors.

COMPETING FINANCIAL INTERESTS: The authors declare no competing financial interests.

\section{REFERENCES}

1. Hell, S. W., \& Wichmann, J. Breaking the diffraction resolution limit by stimulated emission: stimulated-emission-depletion fluorescence microscopy. Optics letters 19(11), 780-782. (1994).

2. Betzig, E. et al. Imaging intracellular fluorescent proteins at nanometer resolution. Science 313, 1642-1645 (2006).

3. Rust, M. J., Bates, M. \& Zhuang, X. Sub-diffraction-limit imaging by stochastic optical reconstruction microscopy (STORM). Nat. Methods 3, 793-796 (2006).

4. Hess, S. T., Girirajan, T. P. \& Mason, M. D. Ultra-high resolution imaging by fluorescence photoactivation localization microscopy. Biophys. J. 91, 4258-4272 (2006).

5. Heilemann, M. et al. Subdiffraction-resolution fluorescence imaging with conventional fluorescent probes. Angew. Chem. Int. Ed. 47, 6172-6176 (2008).

6. Sharonov, A. \& Hochstrasser, R. M. Wide-field subdiffraction imaging by accumulated binding of diffusing probes. Proc. Natl Acad. Sci. USA 103,18911-18916 (2006). 
7. Schnitzbauer, Joerg, et al. Super-resolution microscopy with DNA-PAINT. Nature protocols 12.6:1198. (2017)

8. Juette, M. F. et al. Three-dimensional sub-100 $\mathrm{nm}$ resolution fluorescence microscopy of thick samples. Nat. Methods 5, 527-529 (2008).

9. Huang, B., Wang, W., Bates, M. \& Zhuang, X. Three-dimensional superresolution imaging by stochastic optical reconstruction microscopy. Science 319, 810-813 (2008).

10. Backlund, M. P. et al. Simultaneous, accurate measurement of the 3D position and orientation of single molecules. Proc. Natl Acad. Sci. USA 109, 19087-19092 (2012).

11. Shtengel, G. et al. Interferometric fluorescent super-resolution microscopy resolves 3D cellular ultrastructure. Proc. Natl Acad. Sci. USA 106, 3125-3130 (2009).

12. Aquino, D. et al. Two-color nanoscopy of three-dimensional volumes by 4Pi detection of stochastically switched fluorophores. Nat. Methods 8, 353-359 (2011).

13. Schmidt, R., Wurm, C. A., Jakobs, S., Engelhardt, J., Egner, A., \& Hell, S. W. Spherical nanosized focal spot unravels the interior of cells. Nature methods 5(6), 539-544 (2008).

14. Hell, S. W., Schmidt, R., \& Egner, A. Diffraction-unlimited three-dimensional optical nanoscopy with opposing lenses. Nature Photonics 3(7), 381-387 (2009).

15. Chizhik, A. I., Rother, J., Gregor, I., Janshoff, A. \& Enderlein, J. Metal-induced energy transfer for live cell nanoscopy. Nature Photonics 8, 124-127 (2014).

16. Ghosh, Arindam, et al. Graphene-based metal-induced energy transfer for sub-nanometre optical localization. Nature Photonics 13.12, 860-865. (2019).

17. Balzarotti, F. et al. Nanometer resolution imaging and tracking of fluorescent molecules with minimal photon fluxes. Science 355, 606-612 (2016).

18. Gwosch, K. C., Pape, J. K., Balzarotti, F., Hoess, P., Ellenberg, J., Ries, J., \& Hell, S. W. MINFLUX nanoscopy delivers 3D multicolor nanometer resolution in cells. Nature methods 17(2), 217-224 (2020).

19. Isbaner, S. et al. Axial colocalization of single molecules with nanometer accuracy using metalinduced energy transfer. Nano Lett. 18, 2616-2622 (2018).

20. Karedla, N. et al. Single-molecule metal-induced energy transfer (smMIET): resolving nanometer distances at the single-molecule level. ChemPhysChem 15, 705-711 (2014).

21. Gregor, Ingo, et al. Metal-induced energy transfer. Nanophotonics 8.10 1689-1699. (2019).

22. Simoncelli, S., Makarova, M., Wardley, W. \& Owen, D. M. Toward an axial nanoscale ruler for fluorescence microscopy. ACS Nano 11, 11762-11767 (2017). 
23. Chance, R., Prock, A. \& Silbey, R. Molecular fluorescence and energy transfer near interfaces. Adv. Chem Phys. 37, 1-65 (1978).

24. Enderlein, J. Single-molecule fluorescence near a metal layer. Chem. Phys. 247, 1-9 (1999).

25. Chizhik, Alexey I., and Jörg Enderlein. Metal-Induced Energy Transfer Imaging. In Nanoscale Photonic Imaging, pp. Springer, Cham, 227-239. (2020).

26. Karedla, N. et al. Three-dimensional single-molecule localization with nanometer accuracy using metal-induced energy transfer (MIET) imaging. J. Chem. Phys. 148, 204201 (2018).

27. Moerland, R. J., \& Hoogenboom, J. P. Subnanometer-accuracy optical distance ruler based on fluorescence quenching by transparent conductors. Optica. 3(2), 112-117. (2016).

28. Böhmer, M. \& Enderlein, J. Orientation imaging of single molecules by wide-field epifluorescence microscopy. J. Opt. Soc. Am. B 20, 554-559 (2003).

29. Patra, D., Gregor, I. \& Enderlein, J. Image analysis of defocused single molecule images for three-dimensional molecule orientation studies. J. Phys.Chem.A 108, 6836 (2004).

30. Backer, A. S., \& Moerner, W. E. Determining the rotational mobility of a single molecule from a single image: a numerical study. Optics express, 23(4), 4255-4276 (2015).

31. Chizhik, A. I., Gregor, I., Ernst, B. \& Enderlein, J. Nanocavity-based determination of absolute values of photoluminescence quantum yields. ChemPhysChem 14, 505-513 (2013).

32. Schneider, F., Ruhlandt, D., Gregor, I., Enderlein, J. \& Chizhik, A. I. Quantum yield measurements of fluorophores in lipid bilayers using a plasmonic nanocavity. J. Phys. Chem.Lett. 8, 1472-1475 (2017).

33. Richter, R. P., B'erat, R. \& Brisson, A. R. Formation of solid-supported lipid bilayers: an integrated view. Langmuir 22, 3497-3505 (2006).

34. Meleard, P., Bagatolli, L. A., \& Pott, T. Giant unilamellar vesicle electroformation: From lipid mixtures to native membranes under physiological conditions. Methods in enzymology 465, 161-176 (2009).

35. Bagatolli, L. A. To see or not to see: lateral organization of biological membranes and fluorescence microscopy. Biochim. Biophys. Acta 1758, 1541-1556 (2006).

36. Enderlein, J. and Erdmann, R., Fast fitting of multi-exponential decay curves. Optics Communications, 134 (1-6), pp.371-378 (1997).

37. Enderlein, J., Goodwin, P.M., Van Orden, A., Ambrose, W.P., Erdmann, R. and Keller, R.A., A maximum likelihood estimator to distinguish single molecules by their fluorescence decays. Chemical physics letters, 270 (5-6), pp.464-470 (1997). 
38. Kučerka, N., Nieh, M.-P. \& Katsaras, J. Fluid phase lipid areas and bilayer thicknesses of commonly used phosphatidylcholines as a function of temperature. Biochim. Biophys. Acta 1808, 2761-2771 (2011).

39. Attwood, S. J., Choi, Y. \& Leonenko, Z. Preparation of DOPC and DPPC supported planar lipid bilayers for atomic force microscopy and atomic force spectroscopy. Int. J. Mol. Sci. 14, 3514-3539 (2013).

40. Tristram-Nagle, S., Petrache, H. I. \& Nagle, J. F. Structure and interactions of fully hydrated dioleoylphosphatidylcholine bilayers. Biophys. J. 75, 917-925 (1998).

41. Isbaner, Sebastian, et al. Dead-time correction of fluorescence lifetime measurements and fluorescence lifetime imaging. Optics express 24.9. 9429-9445. (2016). 


\section{Chapter 5}

\section{Single Molecule Spectroscopy and Imaging for Studying Protein Structure and Dynamics}

\subsection{Manuscript: Dimerization of human drebrin-like protein governs its biological activity}

This work [173] outlines the application of FCS and 2fFCS, and of FLIM-FRET imaging to the investigation of protein dimerization and aggregation. In particular, the study focuses on human drebrin-like protein (DBNL) and its dimerization in vivo and in vitro. Additionally, the hydrodynamic radius of DBNL monomer was measured, and the oligomerization properties of three of its truncated variants was investigated. For the dimerization studies, I used a FCCS in vitro assay, while dual-focus FCS was utilized for determining the hydrodynamic radius of DBNL. Oligomerization of DBNL mutants as a function of protein concentration was investigated using classical FCS. For the in vivo assays on the native functionality of DBNL and its mutants, I used FLIM-FRET imaging where EGFP-tagged actin and mCherry labeled DBNL were used as FRET pair. The published report is presented in the next section.

The original research article was published as "Arindam Ghosh, Jörg Enderlein, and Eugenia Butkevich. Dimerization of human drebrin-like protein governs its biological activity. Biochemistry 59(16):1553-1558, 2020." A.G. performed all the FCS, FCCS, dual-focus FCS and FLIM-FRET experiments, did the data analysis and co-wrote the manuscript. 
Reproduced with permission, copyright 2020 American Chemical Society.

The published research article is currently available at https://pubs . acs .org/doi/abs/ 10.1021/acs. biochem.9b01095 


\title{
Dimerization of Human Drebrin-like Protein Governs Its Biological Activity
}

\author{
Arindam Ghosh, Jörg Enderlein, and Eugenia Butkevich*
}

Cite This: Biochemistry 2020, 59, 1553-1558

Read Online

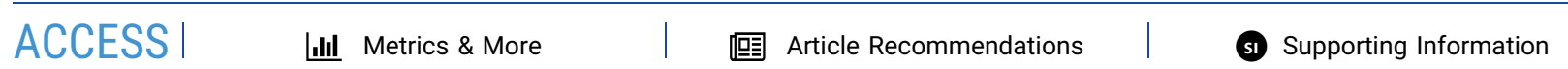

ABSTRACT: Drebrin-like protein (DBNL) is a multidomain F-actin-binding protein, which also interacts with other molecules within different intracellular pathways. Here, we present quantitative measurements on the size and conformation of human DBNL. Using dual-focus fluorescence correlation spectroscopy, we determined the hydrodynamic radius of the DBNL monomer. Native gel electrophoresis and dual-color fluorescence cross-correlation spectroscopy show that both endogenous DBNL and recombinant DBNL exist as dimers under physiological conditions. We demonstrate that C-terminal truncations of DBNL downstream of the coiled-coil domain result in its oligomerization at nanomolar concentrations. In contrast, the ADF-H domain alone is a monomer, which displays a concentration-dependent self-assembly. In vivo FLIM-FRET imaging shows that the presence of only actin-binding domains is not sufficient for DBNL to localize properly at the actin filament inside the cell. In summary, our work provides detailed insight into the structure-function relationship of human drebrin-like protein.

$\mathrm{D}$ rebrin-like protein (also known as DBNL, mAbp1, HIP55, SH3P7, or DBN-1 in Caenorhabditis elegans) is an actin-binding protein of the ADF-H family. Its amino acid sequence consists of an N-terminal ADF-H domain, followed by a coiled-coil region, a proline-rich sequence, and a Cterminal SH3 domain. The multidomain structure of DBNL allows it to serve as an adapter protein for connecting the actin cytoskeleton to many biomolecules enabling multiple cellular functions. Its interaction with F-actin is mediated via the two actin-binding modules: an ADF-H domain and a coiled-coil region. $^{1-3}$ In addition, DBNL interacts with dynamin 1 , WASP-interacting protein WIP, Piccolo, the Cdc42 guanine nucleotide exchange factor Fgd1, FHL2, and other proteins. ${ }^{4-8}$ DBNL is an important player that mediates endocytosis and vesicle recycling. ${ }^{4,-12}$ In addition, it regulates actin dynamics during the formation of dorsal ruffles ${ }^{5}$ and podosomes ${ }^{13}$ as well as during sarcomere contraction. ${ }^{3} \mathrm{~A}$ recent report indicates the role of native $\mathrm{DBNL}$ as a negative regulator of cancer development, while its ADF-H domain alone enhances Rho GTPase signaling and increases the extent of cancer cell invasion. ${ }^{8}$ Atomic force microscopy of C. elegans drebrin-like protein revealed its globular shape. ${ }^{3}$ The crystal structure of the ADF-H domain of Saccharomyces cerevisiae DBNL and the solution NMR structure of the ADF-H domain of human DBNL have been resolved. ${ }^{2,14}$ Recently, a dimeric structure of S. cerevisiae DBNL bound to the Arp2/3 complex has been found using electron microscopy, and its modest propensity to form dimers in solution in the absence of other proteins was suggested. ${ }^{15}$ However, the conformational states in which DBNL exists inside cells remain to be determined. Furthermore, no biophysical measurements of recombinant DBNL using single-molecule spectroscopy have previously been reported.

In this report, we determine the size of human DBNL monomers, examine their self-organization under physiological conditions, and elucidate the impact of the coiled-coil domain in this process. In addition, we demonstrate the importance of the native conformation for the proper interaction of DBNL with actin filaments in cells. For this, we utilize advanced variants of fluorescence correlation spectroscopy (FCS), native gel electrophoresis, and fluorescence lifetime imaging-Förster resonance energy transfer (FLIM-FRET).

To examine the conformational state of endogenous human DBNL, we performed native gel electrophoresis and Western blot analysis of cell lysates (see the Gel electrophoresis and Western blot and Antibodies subsections in the Supporting Information) from four different human cell lines: HeLa (cervix epithelial adenocarcinoma), MCF7 (mammary gland epithelial adenocarcinoma), HEK-293 (embryonic kidney), and hMSCs (human bone marrow-derived mesenchymal stem cells). While the predicted molecular weight of human DBNL is $\sim 48 \mathrm{kDa}$, it migrates as a single band of $\sim 60 \mathrm{kDa}$ under denatured conditions. The retarded protein migration may result from the high content of charged amino acids. ${ }^{9}$ Under native conditions, it migrates as a single band of $\sim 120 \mathrm{kDa}$, which strongly indicates its dimerization (Figure 1A).

This result prompted us to investigate the dimerization of recombinant DBNL (for protein expression and purification details, see the Expression constructs and expression and purification of recombinant proteins subsections in the Supporting Information) using dual-color fluorescence crosscorrelation spectroscopy (FCCS). FCCS is an extension of

Received: December 17, 2019

Revised: April 12, 2020

Published: April 13, 2020 
A.

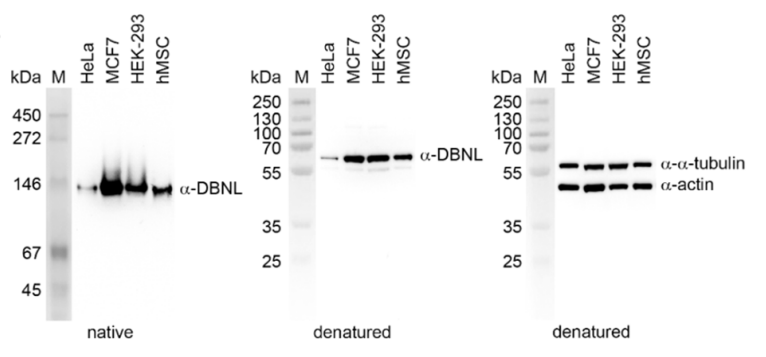

B.
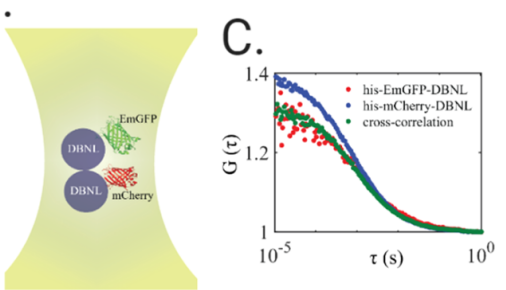

D.

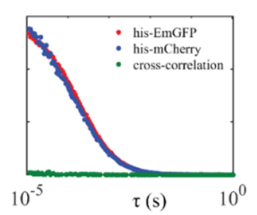

Figure 1. Both endogenous DBNL and recombinant DBNL exist as dimers. (A) DBNL exists as a dimer in human cells. Western blot analysis of DBNL expression in HeLa (cervix epithelial adenocarcinoma) cells, MCF7 (mammary gland epithelial adenocarcinoma) cells, HEK-293 (embryonic kidney) cells, and hMSCs (human bone marrow-derived mesenchymal stem cells). Proteins of total cell lysates were separated by native $10 \%$ Tris-glycins or denatured $12 \%$ Bis-Tris gel electrophoresis, transferred onto a PVDF membrane, and stained with anti-human DBNL antibodies or anti- $\alpha$-tubulin and anti-actin antibodies together for a loading control. Molecular mass markers in kilodaltons are indicated on the left. (B-D) Recombinant DBNL exists as a dimer. (B) Experimental scheme of dual-color fluorescence cross-correlation spectroscopy (FCCS) of an equimolar mixture of his-EmGFP-DBNL and his-mCherry-DBNL. The image shows an illustration of co-diffusion of DBNL monomers tagged with hisEmGFP and his-mCherry in a focused confocal volume. (C) Autocorrelation curves of his-EmGFP-DBNL (red), his-mCherryDBNL (blue), and their cross-correlation (green). (D) Same as panel $\mathrm{C}$ for his-EmGFP and his-mCherry. As one can see in panel $\mathrm{D}$, we observe negligible or no cross-correlation between his-EmGFP and his-mCherry unlike when they are tagged to DBNL as in panel C. All correlation curves were normalized to their amplitudes at time $1 \mathrm{~s}$ when the correlations have decayed completely.

FCS (fluorescence correlation spectroscopy) in which one detects the fluorescence signal from two spectrally distinct fluorescent species simultaneously in two channels (see Figure 1B). ${ }^{16,17} \mathrm{~A}$ subsequent cross-correlation analysis allows the detection of particles carrying both fluorescent labels. The method has been widely exploited to study protein-protein interactions in signaling processes, kinetics of enzymatic cleavage, or dynamic co-localization of proteins in endocytic pathways and intracellular trafficking. ${ }^{18-22}$ We perform FCCS measurements on an equimolar mixture of his-EmGFP-DBNL (DBNL monomer fused with his-tagged emerald green fluorescent protein) and his-mCherry-DBNL (DBNL monomer fused with his-tagged mCherry) monomers (for experimental details, see the Instrumentation and experimental procedures for FCCS, 2fFCS, FCS and FLIM-FRET imaging subsection in the Supporting Information). If EmGFP- and mCherry-labeled monomeric units of DBNL bind to each other, this results in a non-zero fluorescence cross-correlation. Figure 1C shows the fluorescence auto- and cross-correlation curves obtained from a nanomolar mixture of his-EmGFP-
DBNL and his-mCherry-DBNL. We observe a positive crosscorrelation amplitude due to co-diffusion of DBNL monomers. In contrast, we observe negligible or no cross-correlation in the case of only his-EmGFP and his-mCherry (Figure 1D). This finding is in excellent agreement with experiments on endogenous human DBNL. These observations point out that both endogenous DBNL and recombinant human DBNL undergo dimerization under physiological conditions.

We utilized dual-focus FCS (2fFCS) to measure the translational diffusion coefficient of his-EmGFP-DBNL monomers in solution. This value was subsequently converted to the hydrodynamic radius or the radius of hydration of the protein using the Stokes-Einstein relation

$$
D_{T}=\frac{k_{\mathrm{B}} T}{6 \pi \eta R_{\mathrm{H}}}
$$

where $D_{T}$ is the diffusion coefficient at measurement temperature $T, k_{\mathrm{B}}$ is Boltzmann's constant, $\eta$ is the viscosity of the solution, and $R_{\mathrm{H}}$ is the Stokes or hydrodynamic radius. Thus, any change in $R_{\mathrm{H}}$ of a molecule of interest is directly reflected in a change in its translational diffusion coefficient. $2 \mathrm{fFCS}$, in contrast to classical FCS, is not affected by refractive index mismatch, variations in the thickness of the glass coverslip, laser beam astigmatism, or optical saturation of the fluorescent molecules. The technique was introduced in $2007^{23}$ and has been utilized for precise determination of diffusion coefficients of fluorescent molecules at pico- to nanomolar concentrations. ${ }^{24-26}$ Here, we briefly outline the working principle of the technique (for details, see ref 23 and the Instrumentation and experimental procedures for FCCS, 2fFCS, FCS and FLIM-FRET imaging subsection in the Supporting Information). A pair of interleaved pulsing lasers with orthogonal polarization are used for sample excitation. A Nomarski prism in the excitation path between the dichroic mirror and the objective lens deflects the light in a polarization-dependent manner so that after focusing through the objective two laterally shifted but overlapping excitation foci are created. The lateral distance between the foci is wavelength-dependent and has to be determined a priori by a calibration measurement with a dye or fluorescent beads with a known diffusion coefficient. This distance remains unaltered under optical saturation or aberrations and thus can be used as a "ruler" to measure diffusion coefficients. By employing pulsed interleaved excitation (PIE) together with time-correlated single-photon counting (TCSPC), one can determine which detected photon was excited by which laser and thus in which of the two laterally shifted foci. Next, we calculate the fluorescence autocorrelation function (ACF) of each focus and the cross-correlation function (CCF) between foci and perform a diffusion coefficient global fit of the ACF and CCF using appropriate model functions. ${ }^{23}$ For our measurements on DBNL, we used a 100 pM his-EmGFP-DBNL solution in $1 \times$ PBS ( $\mathrm{pH} 7.4$ ) and employed a commercial confocal microscope for the $2 \mathrm{fFCS}$ experiments. Figure 2 shows the ACFs and CCFs that were then fitted globally, resulting in the following fit parameters: diffusion coefficient $D$ $=37.50 \pm 2.50 \mu \mathrm{m}^{2} / \mathrm{s}$, triplet state relaxation time $\tau_{\mathrm{T}}=152.2$ $\pm 3.5 \mu \mathrm{s}$, laser-focus beam waist diameter $\omega_{0}=435 \mathrm{~nm}$, and Rayleigh length $a_{0}=203 \mathrm{~nm}$. Using eq 1 , we calculated a Stokes radius of his-EmGFP-DBNL of $6.72 \pm 0.40 \mathrm{~nm}$. Next, we quantified the diffusion coefficient of his-EmGFP only $(D=$ $104.20 \pm 8.50 \mu \mathrm{m}^{2} / \mathrm{s}$ ), which yields an $R_{\mathrm{H}}$ value of $2.42 \pm 0.20$ 


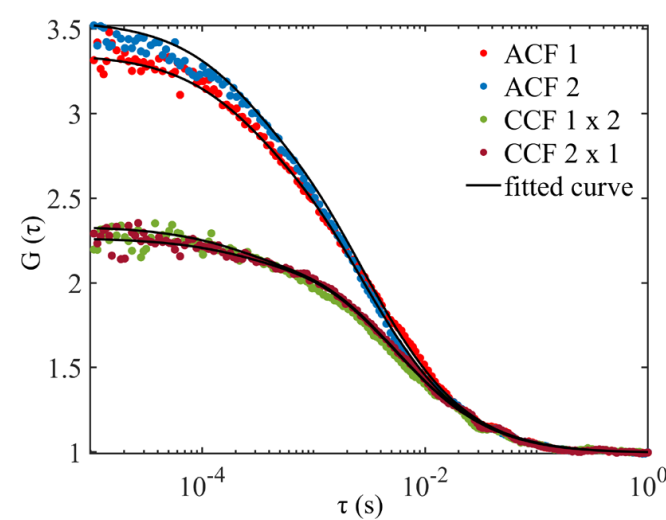

Figure 2. 2fFCS measurement of a $100 \mathrm{pM}$ aqueous solution of hisEmGFP-DBNL. Autocorrelation functions (ACFs) are shown as red circles for the first focus (ACF 1) and blue circles (ACF 2) for the second focus. The two possible cross-correlation functions (CCFs) between both foci are represented as green and brown circles, respectively. Solid lines indicate a global fit. As fit parameters, we obtained diffusion coefficient $D=37.50 \pm 2.50 \mu \mathrm{m}^{2} / \mathrm{s}$, triplet state relaxation time $\tau_{\mathrm{T}}=152.2 \pm 3.5 \mu \mathrm{s}, \omega_{0}=435 \mathrm{~nm}$, and $a_{0}=203 \mathrm{~nm}$. All correlation curves were normalized to their amplitudes at $1 \mathrm{~s}$. The excitation intensity was $10 \mu \mathrm{W}$ for each laser.

$\mathrm{nm}$ that matches the previously reported value. ${ }^{27}$ To estimate the $R_{\mathrm{H}}$ value of DBNL monomers without tagged his-EmGFP, we proceeded as follows. Assuming a spherical shape for the protein, we obtained volumes of $1270 \pm 230 \mathrm{~nm}^{3}$ for hisEmGFP-DBNL and $59 \pm 14 \mathrm{~nm}^{3}$ for his-EmGFP. The difference yields a volume of $1212 \pm 200 \mathrm{~nm}^{3}$ for DBNL, which corresponds to a hydrodynamic radius of $6.60 \pm 0.40$ $\mathrm{nm}$. It should be mentioned that this value will be slightly larger than that for pure DBNL due to the presence of the fusion linker with a length of 14 amino acids.

The shape of a protein is specified by noncovalent interactions between regions of its amino acid sequence. Mutations of proteins are well-known to destabilize their conformation and initiate oligomerization and even aggregation, which might cause cellular dysfunction. ${ }^{28-30} \mathrm{~A}$ twostranded $\alpha$-helical coiled coil is a most common structural motif that mediates dimerization via hydrophobic and electrostatic interactions between residues. ${ }^{31,32}$ To examine the impact of the coiled-coil domain in the dimerization of DBNL, we investigated three DBNL truncation mutants: DBNL(1-179) (truncated before the coiled-coil domain), DBNL(1-256) (truncated after the coiled-coil domain) and DBNL(1-374) (deleted SH3 domain) (Figure 3A). The nontagged truncated proteins were expressed in MCF7 cells under the control of a CMV promoter, and cell lysates were subjected to native gel electrophoresis and Western blot analysis. Surprisingly, none of the mutated proteins displayed a monomeric or dimeric structure. Instead, they migrated through the native gel significantly slower than the full-length DBNL (Figure 3B). Similarly, the native recombinant hisEmGFP-tagged truncated proteins showed smeared patterns with molecular weights that were higher than that of full-length his-EmGFP-DBNL (Figure 3C,D). These results clearly indicate that truncations of DBNL lead to a change in compaction and might induce oligomerization.

To probe the oligomerization of truncated DBNL, we utilized classical FCS and measured the translational diffusion times of recombinant his-EmGFP-tagged DBNL(1-179),

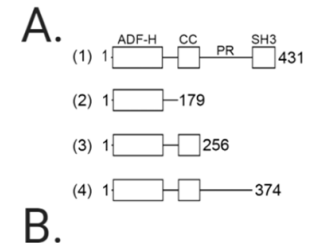
C.

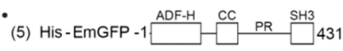 (6) His- EmGFP - 1 $\square-179$ (7) His - EMGFP - $1 \square \square \square 256$ (8) His-EmGFP -1 $\square \square \square-374$

D.
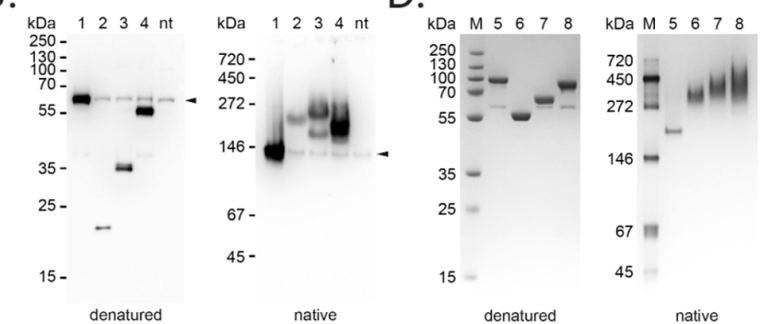

E.
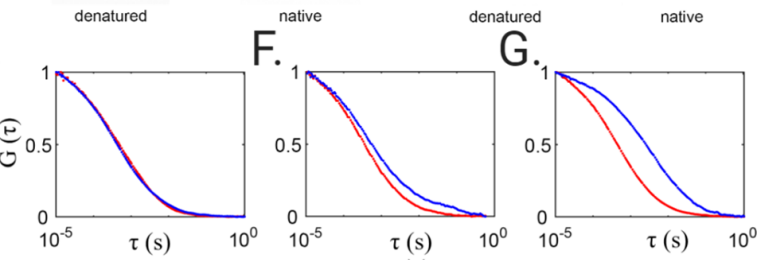

Figure 3. Biophysical properties of truncated DBNL. (A) Schematic representation of full-length DBNL and its truncation mutants: DBNL(1-179), DBNL(1-256), and DBNL(1-374). (B) Western blot analysis of DBNL in MCF7 cells transfected with full-length DBNL or its truncation mutants, indicated in panel $\mathrm{A}$, and in nontransfected cells (nt). Denatured or native proteins were separated by $12 \%$ Bis-Tris or $10 \%$ Tris-glycine gel electrophoresis, respectively, transferred onto a PVDF membrane, and stained with anti-human DBNL antibodies. Molecular mass markers in kilodaltons are indicated at the left. The low-intensity bands indicated by arrowheads are of endogenous DBNL. The weak signal of DBNL $(1-179)$ is due to the reduced number of binding sites for the polyclonal antibodies. (C) Schematic representation of recombinant his-EmGFP-tagged fulllength DBNL and its truncation mutants: DBNL(1-179), DBNL(1256), and DBNL(1-374). (D) Denatured or native proteins, indicated in panel C, were separated by $12 \%$ Bis-Tris or $10 \%$ Trisglycine gel electrophoresis, respectively, and stained with Roti-Blue quick. Molecular mass markers in kilodaltons are indicated on the left. (E-G) FCS measurements of DBNL truncated at three different positions. (E) Normalized fluorescence autocorrelation of hisEmGFP-DBNL(1-179). Autocorrelation curves for $100 \mathrm{pM}$ and 10 $\mathrm{nM}$ solutions of the protein are colored red and blue, respectively. As one can see, there is a negligible difference in the diffusion time scales for $100 \mathrm{pM}$ and $10 \mathrm{nM}$. (F) Same as panel E for his-EmGFPDBNL(1-256). The diffusion speed decreases for $10 \mathrm{nM}$ (blue) compared to $100 \mathrm{pM}$ protein (red). (G) Same as panel E for hisEmGFP-DBNL(1-374). Protein oligomerization leads to slower diffusion at $10 \mathrm{nM}$ (blue) relative to $100 \mathrm{pM}$ (red) protein.

DBNL(1-256), and DBNL(1-374) at $100 \mathrm{pM}$ and $10 \mathrm{nM}$ concentrations in $1 \times \mathrm{PBS}$ ( $\mathrm{pH} 7.4)$ using a laser intensity of 10 $\mu \mathrm{W}$ (see the Instrumentation and experimental procedures for FCCS, 2fFCS, FCS and FLIM-FRET imaging and Data evaluation subsections in the Supporting Information). hisEmGFP-DBNL(1-179) showed the same diffusion times at $100 \mathrm{pM}$ and $10 \mathrm{nM}$ concentrations (Figure 3E). In contrast, diffusion times of both his-EmGFP-DBNL(1-256) and hisEmGFP-DBNL(1-374) decreased significantly at $10 \mathrm{nM}$ compared to $100 \mathrm{pM}$ concentration (Figure 3F,G). Thus, it can be inferred that at a concentration of $10 \mathrm{nM}$ the isolated ADF-H domain exists as a monomer, while both mutants truncated after the coiled-coil domain undergo oligomerization at the same concentration. These results indicate that the 
coiled-coil domain mediates self-assembly, but the presence of both proline-rich and $\mathrm{SH} 3$ domains is essential for DBNL to maintain a stable dimer conformation. Interestingly, while we do not observe oligomerization of his-DBNL(1-179) at nanomolar concentrations in our FCS measurements, it migrates as an oligomer through the native gel when taken at micromolar concentrations. We suppose that the oligomerization of the ADF-H domain of DBNL is concentrationdependent, similar to that of human cofilin, which consists of a single ADF-H domain and displays a concentration-dependent self-assembly via its C-terminal part. ${ }^{33,34}$

Previously, we used atomic force microscopy to show that drebrin-like protein has a globular shape and decorates the sides of actin filaments. ${ }^{3}$ Using an in vitro actin binding assay, Kessels and colleagues identified two independent actinbinding modules within the structure of the mammalian DBNL: ADF-H and coiled-coil domains. ${ }^{1}$ In a two-component in vitro system, DBNL truncated after a coiled-coil domain binds to F-actin as strongly as the full-length protein, while the isolated ADF-H domain binds with a significantly reduced affinity. ${ }^{1}$ To probe the impact of protein conformation on its interaction with F-actin inside cells, we utilized FLIM-FRET imaging. ${ }^{35-37}$ In FLIM-FRET, one labels the target molecule of interest with a donor, and the corresponding ligand with an acceptor, and then determines the FRET efficiency between the donor and acceptor by measuring the donor fluorescence lifetime. Due to the short nanometer range of FRET, a high FRET efficiency indicates direct target-ligand binding. A reasonable control experiment is to measure the fluorescence lifetime of the donor in the absence of the acceptor or by remeasuring the donor lifetime after full acceptor photobleaching. For our purpose, we co-expressed EGFP (enhanced GFP)-actin (donor) and mCherry-DBNL or mCherry-tagged truncated DBNL mutants (acceptor) in MCF7 cells (see the Cell culture and transfection and Antibodies subsections in the Supporting Information for sample preparation details) and measured the fluorescence lifetime of EGFP-actin (see the FLIM-FRET Imaging subsection in the Supporting Information for experimental details). Experiments were performed using a commercial confocal microscope upgraded with timecorrelated single-photon counting (TCSPC) for fluorescence lifetime determination. FLIM images of individual cells were recorded by focusing the laser beam on areas spanning around $\sim 40 \mu \mathrm{m} \times 40 \mu \mathrm{m}$. Recorded TCSPC histograms were then fitted with a biexponential decay function, and a compound fluorescence lifetime value was calculated as the average of the two found decay times weighted with their corresponding fit amplitudes. Figure 4A shows fluorescence lifetime images of EGFP-actin in the presence of full-length mCherry-tagged DBNL in five representative cells. For all of the cells with fulllength protein, the lifetime was found to be $1.87 \pm 0.05 \mathrm{~ns}$ (see the leftmost data in Figure 4F). In contrast, the lifetime values of EGFP-actin in the presence of mCherry-tagged DBNL(1179) (Figure 4B), DBNL(1-276) (Figure 4C), and DBNL(1-374) (Figure 4D) were found to be $2.29 \pm 0.06$, $2.28 \pm 0.04$, and $2.32 \pm 0.04 \mathrm{~ns}$, respectively (errors in lifetime values indicate the standard error of the mean). Finally but importantly, the lifetime value for "donor-only" cells expressing only EGFP-actin (Figure 4E) is $2.34 \pm 0.05 \mathrm{~ns}$, matching closely the values that we obtained for cells expressing truncated DBNL.

Thus, FLIM-FRET experiments show the decrease in the fluorescence lifetime of EGFP-actin inside cells in the presence

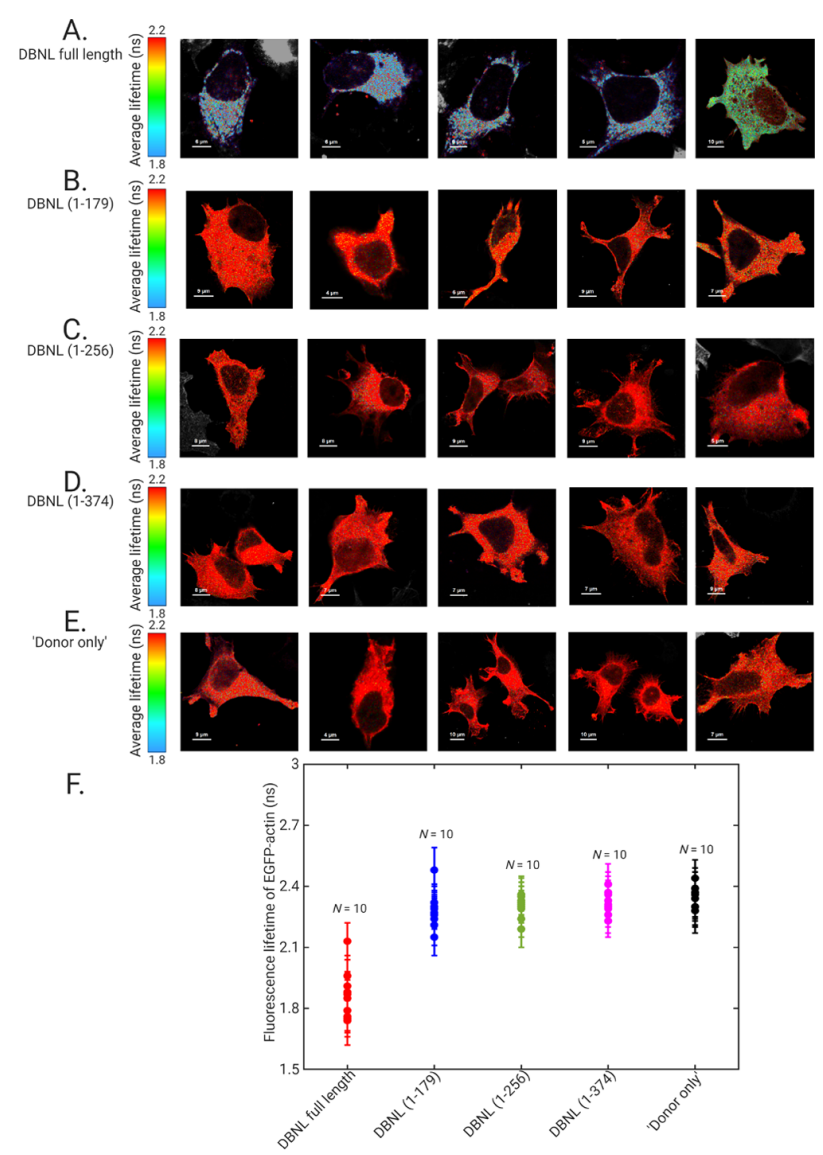

Figure 4. Interaction of EGFP-actin and mCherry-DBNL and its truncation mutants measured using FLIM-FRET imaging. (A) MCF7 cells were co-transfected with EGFP-actin (donor) and mCherryDBNL (acceptor). Five representative FLIM images of EGFP-actin are shown. The false color scale represents the average fluorescence lifetime values. (B) Same as panel A for EGFP-actin co-transfected with mCherry-DBNL(1-179). (C) Same as panel A for EGFP-actin co-transfected with mCherry-DBNL(1-256). (D) Same as panel A for EGFP-actin co-transfected with mCherry-DBNL(1-374). (E) FLIM images of "donor-only" cells expressing EGFP-actin alone. (F) A comparative plot shows mean fluorescence lifetime values and the square root of variance (error bars) of EGFP-actin in individual cells in the presence of DBNL (red), DBNL(1-179) (blue), DBNL(1256) (green), DBNL(1-374) (magenta), and "donor only" (black). Each statistical group consists of 10 cells as indicated by $N$.

of mCherry-DBNL, indicating a strong interaction of both proteins. The absence of a FRET-induced lifetime reduction of EGFP-actin in the presence of truncated mutants of DBNL suggests an absence of interaction (Figure 4F). It can be possible that other actin-binding proteins with higher F-actin binding affinity displace truncated DBNL from actin filaments in live cells. Previous reports demonstrate the importance of the native structure of DBNL for retaining its biological activity. ${ }^{3,5,8,38,39}$ In cells, DBNL undergoes proteolysis by the ubiquitous calcium-sensitive protease calpain-2, which cleaves DBNL between the coiled coil and the proline-rich region. ${ }^{5}$ Here, the $\mathrm{N}$-terminal fragment (consisting of two actin-binding modules) alone cannot rescue formation of actin-based dorsal ruffles in DBNL-deficient cells. ${ }^{5}$ The expression of the ADF-H domain alone is known to enhance Rho GTPase signaling and to induce breast cancer cell invasion. ${ }^{8}$ In C. elegans, the impact 
of truncations can be visually evaluated by the change in the worm's movement ability. While the dbn-1(vit7) mutant, containing a premature stop codon after the largest part of the ADF-H domain, displays a strong body curvature and a striking hyperbending phenotype, the $d b n-1$ (ok925) mutant truncated after two coiled coils moves in sinusoidal waves similar to the wild type. ${ }^{38}$ However, the ok925 gene has a mild disorganization of actin filaments during body-wall muscle contraction $^{3}$ and defective vesicle scission during endocytosis in the intestines. ${ }^{39}$ Taken together, our data demonstrate that the amino acid sequence of DBNL defines its existence as a dimer and that DBNL can efficiently interact with actin filaments inside a cell only in its native conformation.

\section{ASSOCIATED CONTENT}

\section{S) Supporting Information}

The Supporting Information is available free of charge at https://pubs.acs.org/doi/10.1021/acs.biochem.9b01095.

Expression constructs, expression and purification of recombinant proteins, antibodies, cell culture and transfection, gel electrophoresis and Western blot, instrumentation and experimental procedures for FCCS, 2fFCS, FCS, and FLIM-FRET imaging, and data evaluation (PDF)

\section{Accession Codes}

Drebrin-like protein isoform a, NP_054782.2.

\section{AUTHOR INFORMATION}

\section{Corresponding Author}

Eugenia Butkevich - Third Institute of Physics-Biophysics, Georg August University, 37077 Göttingen, Germany; (1) orcid.org/0000-0002-3834-7793;

Email: eugenia.butkevich@phys.uni-goettingen.de

\section{Authors}

Arindam Ghosh - Third Institute of Physics-Biophysics, Georg August University, 37077 Göttingen, Germany

Jörg Enderlein - Third Institute of Physics-Biophysics, Georg August University, 37077 Göttingen, Germany; 이이.org/ 0000-0001-5091-7157

Complete contact information is available at: https://pubs.acs.org/10.1021/acs.biochem.9b01095

\section{Author Contributions}

A.G. performed the FCS, FCCS, 2fFCS, and FLIM-FRET experiments, performed data analysis, and co-wrote the manuscript. J.E. co-wrote the manuscript and helped in data analysis. E.B. designed the project, generated the constructs, performed cell culture and biochemical experiments, and cowrote the manuscript.

\section{Funding}

The authors are grateful to the Deutsche Forschungsgemeinschaft (DFG) for financial support via Project A06 of the Collaborative Research Center SFB 860.

\section{Notes}

The authors declare no competing financial interest.

\section{ACKNOWLEDGMENTS}

E.B. thanks Tanja Gall for her help and assistance.

\section{REFERENCES}

(1) Kessels, M. M., Engqvist-Goldstein, Å. E., and Drubin, D. G. (2000) Association of mouse actin-binding protein 1 (mAbp1/ SH3P7), an Src kinase target, with dynamic regions of the cortical actin cytoskeleton in response to Racl activation. Mol. Biol. Cell 11, 393-412.

(2) Quintero-Monzon, O., Rodal, A. A., Strokopytov, B., Almo, S. C., and Goode, B. L. (2005) Structural and functional dissection of the Abp1 ADFH actin-binding domain reveals versatile in vivo adapter functions. Mol. Biol. Cell 16, 3128-39.

(3) Butkevich, E., Bodensiek, K., Fakhri, N., Von Roden, K., Schaap, I. A., Majoul, I., Schmidt, C. F., and Klopfenstein, D. R. (2015) Drebrin-like protein DBN-1 is a sarcomere component that stabilizes actin filaments during muscle contraction. Nat. Commun. 6, 7523.

(4) Kessels, M. M., Engqvist-Goldstein, Å. E., Drubin, D. G., and Qualmann, B. (2001) Mammalian Abp1, a signal-responsive F-actinbinding protein, links the actin cytoskeleton to endocytosis via the GTPase dynamin. J. Cell Biol. 153, 351-366.

(5) Cortesio, C. L., Perrin, B. J., Bennin, D. A., and Huttenlocher, A. (2010) Actin-binding protein-1 interacts with WASp-interacting protein to regulate growth factor-induced dorsal ruffle formation. Mol. Biol. Cell 21, 186-197.

(6) Fenster, S. D., Kessels, M. M., Qualmann, B., Chung, W. J., Nash, J., Gundelfinger, E. D., and Garner, C. C. (2003) Interactions between Piccolo and the actin/dynamin-binding protein Abp1 link vesicle endocytosis to presynaptic active zones. J. Biol. Chem. 278, 2026820277.

(7) Hou, P., Estrada, L., Kinley, A. W., Parsons, J. T., Vojtek, A. B., and Gorski, J. L. (2003) Fgd1, the Cdc42 GEF responsible for Faciogenital Dysplasia, directly interacts with cortactin and mAbp1 to modulate cell shape. Human molecular genetics 12, 1981-1993.

(8) Boateng, L. R., Bennin, D., De Oliveira, S., and Huttenlocher, A. (2016) Mammalian Actin-binding Protein-1/Hip-55 Interacts with FHL2 and Negatively Regulates Cell Invasion. J. Biol. Chem. 291, 13987-13998.

(9) Larbolette, O., Wollscheid, B., Schweikert, J., Nielsen, P. J., and Wienands, J. (1999) SH3P7 is a cytoskeleton adapter protein and is coupled to signal transduction from lymphocyte antigen receptors. Mol. Cell. Biol. 19, 1539-1546.

(10) Le Bras, S., Foucault, I., Foussat, A., Brignone, C., Acuto, O., and Deckert, M. (2004) Recruitment of the actin-binding protein HIP-55 to the immunological synapse regulates $\mathrm{T}$ cell receptor signaling and endocytosis. J. Biol. Chem. 279, 15550-15560.

(11) Connert, S., Wienand, S., Thiel, C., Krikunova, M., Glyvuk, N., Tsytsyura, Y., Hilfiker-Kleiner, D., Bartsch, J. W., Klingauf, J., and Wienands, J. (2006) SH3P7/mAbp1 deficiency leads to tissue and behavioral abnormalities and impaired vesicle transport. EMBO J. 25, $1611-1622$.

(12) He, K., Xing, R., Yan, X., Tian, A., Zhang, M., Yuan, J., Lv, Z., Fang, X., Li, Z., and Zhang, Y. (2015) Mammalian actin-binding protein 1/HIP-55 is essential for the scission of clathrin-coated pits by regulating dynamin-actin interaction. FASEB J. 29, 2495-2503.

(13) Boateng, L. R., Cortesio, C. L., and Huttenlocher, A. (2012) Src-mediated phosphorylation of mammalian Abp1 (DBNL) regulates podosome rosette formation in transformed fibroblasts. J. Cell Sci. 125, 1329-1341.

(14) Goroncy, A. K., Koshiba, S., Tochio, N., Tomizawa, T., Sato, M., I noue, M., Watanabe, S., Hayashizaki, Y., Tanaka, A., Kigawa, T., and Yokoyama, S. (2009) NMR solution structures of actin depolymerizing factor homology domains. Protein Sci. 18, 2384-92.

(15) Guo, S., Sokolova, O. S., Chung, J., Padrick, S., Gelles, J., and Goode, B. L. (2018) Abp1 promotes Arp2/3 complex-dependent actin nucleation and stabilizes branch junctions by antagonizing GMF. Nat. Commun. 9, 2895.

(16) Eigen, M., and Rigler, R. (1994) Sorting single molecules: application to diagnostics and evolutionary biotechnology. Proc. Natl. Acad. Sci. U. S. A. 91, 5740-5747. 
(17) Schwille, P., Meyer-Almes, F.-J., and Rigler, R. (1997) Dualcolor fluorescence cross-correlation spectroscopy for multicomponent diffusional analysis in solution. Biophys. J. 72, 1878-1886.

(18) Bacia, K., Majoul, I. V., and Schwille, P. (2002) Probing the endocytic pathway in live cells using dual-color fluorescence crosscorrelation analysis. Biophys. J. 83, 1184-1193.

(19) Bacia, K., and Schwille, P. (2003) A dynamic view of cellular processes by in vivo fluorescence auto-and cross-correlation spectroscopy. Methods 29, 74-85.

(20) Kettling, U., Koltermann, A., Schwille, P., and Eigen, M. (1998) Real-time enzyme kinetics monitored by dual-color fluorescence cross-correlation spectroscopy. Proc. Natl. Acad. Sci. U. S. A. 95, 1416-1420.

(21) Kohl, T., Haustein, E., and Schwille, P. (2005) Determining protease activity in vivo by fluorescence cross-correlation analysis. Biophys. J. 89, 2770-2782.

(22) Baudendistel, N., Müller, G., Waldeck, W., Angel, P., and Langowski, J. (2005) Two-hybrid fluorescence cross-correlation spectroscopy detects protein-protein interactions in vivo. ChemPhysChem 6, 984-990.

(23) Dertinger, T., Pacheco, V., von der Hocht, I., Hartmann, R., Gregor, I., and Enderlein, J. (2007) Two-focus fluorescence correlation spectroscopy: A new tool for accurate and absolute diffusion measurements. ChemPhysChem 8, 433-443.

(24) Weiß, K., and Enderlein, J. (2012) Lipid Diffusion within Black Lipid Membranes Measured with Dual-Focus Fluorescence Correlation Spectroscopy. ChemPhysChem 13, 990-1000.

(25) Weiß, K., Neef, A., Van, Q., Kramer, S., Gregor, I., and Enderlein, J. (2013) Quantifying the diffusion of membrane proteins and peptides in black lipid membranes with 2-focus fluorescence correlation spectroscopy. Biophys. J. 105, 455-462.

(26) Dertinger, T., Loman, A., Ewers, B., Müller, C. B., Krämer, B., and Enderlein, J. (2008) The optics and performance of dual-focus fluorescence correlation spectroscopy. Opt. Express 16, 14353-14368.

(27) Bhunia, D., Chowdhury, R., Bhattacharyya, K., and Ghosh, S. (2015) Fluorescence fluctuation of an antigen-antibody complex: circular dichroism, FCS and smFRET of enhanced GFP and its antibody. Phys. Chem. Chem. Phys. 17, 25250-25259.

(28) Kelly, J. W. (1996) Alternative conformations of amyloidogenic proteins govern their behavior. Curr. Opin. Struct. Biol. 6, 11-17.

(29) Dobson, C. M. (1999) Protein misfolding, evolution and disease. Trends Biochem. Sci. 24, 329-332.

(30) Jang, H., Hall, C. K., and Zhou, Y. (2004) Assembly and kinetic folding pathways of a tetrameric $\beta$-sheet complex: molecular dynamics simulations on simplified off-lattice protein models. Biophys. J. 86, $31-49$.

(31) Kammerer, R. A., Schulthess, T., Landwehr, R., Lustig, A., Engel, J., Aebi, U., and Steinmetz, M. O. (1998) An autonomous folding unit mediates the assembly of two-stranded coiled coils. Proc. Natl. Acad. Sci. U. S. A. 95, 13419-13424.

(32) Drobnak, I., Gradisar, H., Ljubetic, A., Merljak, E., and Jerala, R. (2017) Modulation of Coiled-Coil Dimer Stability through Surface Residues while Preserving Pairing Specificity. J. Am. Chem. Soc. 139, 8229-8236.

(33) Pfannstiel, J., Cyrklaff, M., Habermann, A., Stoeva, S., Griffiths, G., Shoeman, R., and Faulstich, H. (2001) Human cofilin forms oligomers exhibiting actin bundling activity. J. Biol. Chem. 276, 49476-84.

(34) Goyal, P., Pandey, D., Brunnert, D., Hammer, E., Zygmunt, M., and Siess, W. (2013) Cofilin oligomer formation occurs in vivo and is regulated by cofilin phosphorylation. PLoS One 8, e71769.

(35) Wallrabe, H., and Periasamy, A. (2005) Imaging protein molecules using FRET and FLIM microscopy. Curr. Opin. Biotechnol. 16, 19-27.

(36) Jares-Erijman, E. A., and Jovin, T. M. (2006) Imaging molecular interactions in living cells by FRET microscopy. Curr. Opin. Chem. Biol. 10, 409-416.
(37) Wouters, F. S., and Bastiaens, P. I. (2000) Imaging proteinprotein interactions by fluorescence resonance energy transfer (FRET) microscopy. Curr. Protoc. Cell Biol. 7, 17.

(38) Butkevich, E., Weist, P., Härtter, D., Klopfenstein, D. R., Garces, R., and Schmidt, C. F. (2019) Drebrin-like protein regulates body bending of $\mathrm{C}$. elegans via suppression of NCA cation leak channels. bioRxiv, DOI: 10.1101/2019.12.12.874289.

(39) Shi, X., Duan, F., Lin, L., Xu, Q., Xu, T., and Zhang, R. (2019) WIP-1 and DBN-1 promote scission of endocytic vesicles by bridging actin and Dynamin-1 in the C. elegans intestine. J. Cell Sci. 132, jcs228023. 


\title{
Supporting Information : Dimerization of
}

\section{Human Drebrin-like Protein Governs its}

\section{Biological Activity}

\author{
Arindam Ghosh, Jörg Enderlein, and Eugenia Butkevich* \\ III. Institute of Physics, Biophysics, Georg August University, Friedrich-Hund-Platz 1, \\ 37077 Göttingen, Germany \\ *E-mail: eugenia.butkevich@phys.uni-goettingen.de
}




\section{Materials and Methods}

\section{Expression constructs}

A cDNA encoding human DBNL was amplified by PCR from Human Brain Whole QUICKClone $^{\mathrm{TM}}$ cDNA (Clontech Laboratories) using the primers: attB1DBNL forward 5'-GGGG ACAAGTTTGTACAAAAAAGCAGGCTCGATGGCGGCGAACCTGAGCCG-3' and attB2-DBNL reverse 5'-GGGGACCACTTTGTACAAGAAAGCTGGGTGTCACTCAAT GAGCTCCACGT-3', subcloned into pDONR201 (Invitrogen) and subsequently cloned into CMV-mCherry-Gateway vector or Vivid Colors ${ }^{\mathrm{TM}}$ pcDNA $^{\mathrm{TM}} 6.2 / \mathrm{N}-$ EmGFP-DEST vector (ThermoFisher Scientific) to obtain CMV-mCherry-DBNL or CMV-EmGFP-DBNL, respectively. To create a CMV-mCherry-Gateway vector, mCherry was amplified from pmCherryN1 vector (Clontech Laboratories) and placed between XhoI and XbaI sites to exchange EmGFP in pcDNA ${ }^{\mathrm{TM}} 6.2$ /N-EmGFP-DEST. cDNAs encoding DBNL, mCherry-DBNL, Em GFP-DBNL, mCherry and EmGFP alone as well as EmGFP-tagged DBNL(1-179), DBNL(1256) and DBNL(1-374) were amplified by PCR and cloned into pET28a vector (Novagen) between BamHI and NotI sites to obtain constructs for expression of his-tagged proteins in E. coli. CMV-Gateway vector was created by excision of EmGFP from $\operatorname{pcDNA}^{\mathrm{TM}} 6.2 / \mathrm{N}-$ EmGFP-DEST between two XhoI sites. cDNAs encoding DBNL, DBNL(1-179), DBNL(1256) and DBNL(1-374) were amplified by PCR and cloned into CMV-Gateway vector and into CMV-mCherry-Gateway vector for expression in MCF7 cells. All cDNA clones were confirmed by DNA sequencing at SeqLab. pEGFP-Actin (6116-1) was purchased from Clontech Laboratories.

\section{Expression and purification of recombinant proteins}

E. coli BL21-Gold (Agilent Technologies) were transformed with pET28a-mCherry-DBNL, pET28a-EmGFP-DBNL, pET28a-EmGFP-DBNL(1-179), pET28a-EmGFP-DBNL(1-256), pET28a-EmGFP-DBNL(1-374), pET28a-EmGFP, or pET28a-mCherry. Cells were grown 
to an $\mathrm{OD}_{600}$ of 0.4 in in Luria-Bertani medium containing $50 \mathrm{mg} / \mathrm{l} \mathrm{kanamycin} \mathrm{at} 37^{\circ} \mathrm{C}$. Cells were induced to express protein by the addition of $0.1 \mathrm{mM} \mathrm{IPTG}$, and incubation continued for a further $4-5 \mathrm{~h}$ at $22{ }^{\circ} \mathrm{C}$. Cultures were centrifuged at $4,600 \mathrm{~g}$ for $20 \mathrm{~min}$ at $4{ }^{\circ} \mathrm{C}$. Pellets were suspended in $20 \mathrm{ml}$ of lysis buffer containing $20 \mathrm{mM} \mathrm{NaH}_{2} \mathrm{PO}_{4} \mathrm{pH}$ 8.0, $500 \mathrm{mM} \mathrm{NaCl}, 10$ $\mathrm{mM} \beta$-mercaptoethanol, $1 \mathrm{mg} / \mathrm{ml}$ lysozyme, $0.01 \mathrm{mg} / \mathrm{ml}$ DNAse and protease inhibitor cocktail (S8830, Sigma-Aldrich) and subjected to three times alternating procedures of freezing in liquid nitrogen followed by thawing on ice. Samples were sonicated 4 times 20 second each on melting ice with intervals for cooling using a Branson model 250 sonifier. Lysates were spun for a further $20 \mathrm{~min}$ at 40,000 g at $4{ }^{\circ} \mathrm{C}$ and resulting supernatants were incubated 1 hour with $\mathrm{Ni}^{2+}$-nitrilotriacetate (NTA) agarose beads (QIAGEN) at $4{ }^{\circ} \mathrm{C}$ on rotating wheel. Beads were washed in buffer containing $20 \mathrm{mM} \mathrm{NaH}_{2} \mathrm{PO}_{4} \mathrm{pH}$ 8.0, $500 \mathrm{mM} \mathrm{NaCl}, 10 \mathrm{mM}$ $\beta$-mercaptoethanol, $40 \mathrm{mM}$ imidazole, 1mM PMSF. Protein was eluted with buffer containing $20 \mathrm{mM} \mathrm{NaH}_{2} \mathrm{PO}_{4} \mathrm{pH}$ 8.0, $500 \mathrm{mM} \mathrm{NaCl}, 10 \mathrm{mM} \beta$-mercaptoethanol, $350 \mathrm{mM}$ imidazole, 1mM PMSF. Elution buffer was exchanged to PBS pH 7.4 (D8537, Sigma-Aldrich) using Amicon Centrifugal Filters (Merck).

\section{Antibodies}

Antibodies specific for DBNL were raised in chicken against his-tagged full-length human DBNL coupled to keyhole limpet hemocyanin at Bioscience. The final antisera was purified using NHS-activated Sepharose High Performance (GE Healthcare Life Science). The reason for generation of these antibodies was that two tested anti-DBNL antibodies (sc398351, Santa Cruz and HPA020265, Sigma-Aldrich) recognized a denatured but not a native form of DBNL and any antibodies, which recognize native human DBNL were reported so far. The antibodies were validated in western blot. The immunoreactivity was significantly reduced upon treatment of MCF7 cells with shRNA against human DBNL (sc-75255-SH, Santa Cruz Biotechnology). The band was not detected by this antibodies preincubated with his-DBNL and by preimmune serum subjected to purification in parallel to postimmune serum. As 
seen in Fig. 3B, these antibodies recognize full-length DBNL and its truncation mutants expressed from the plasmids. The signal from the truncated construct DBNL(1-179) is reduced presumably due to the reduction of number of antibody-binding sites.

\section{Cell culture and transfection}

MCF7, HEK-293 and HeLa cells were purchased from ATCC and hMSC from Lonza. All cell lines tested negative for micoplasma contamination. Cells were cultured in DMEM supplemented with 10\% FCS, 2 mM L-glutamine, 1 mM sodium pyruvate, $100 \mathrm{U} / \mathrm{ml}$ penicillinstreptomycin in a humidified $5 \% \mathrm{CO}_{2}$ atmosphere at $37{ }^{\circ} \mathrm{C}$. For western blot, MCF7 cells were transfected with non-tagged DBNL, DBNL(1-179), DBNL(1-256) or DBNL(1-374) using ViaFect (Promega) and assayed $24 \mathrm{~h}$ later. For FLIM-FRET imaging, MCF7 cells were transfected with EGFP-actin alone or co-transfected with EGFP-actin and mCherry-DBNL, mCherry-DBNL(1-179), mCherry-DBNL(1-256) or mCherry-DBNL(1-374). 12 hours later cells were rinsed with PBS and fixed in $4 \%$ (v/v) formaldehyde/PBS for 15 min. Samples were washed in PBS, rinsed with distilled water, and mounted using Fluoroshield ${ }^{\mathrm{TM}}$ (F6182, Sigma-Aldrich).

\section{Gel electrophoresis and Western blot}

About 200,000 cells were lysed in $100 \mu$ l of lysis buffer, containing $150 \mathrm{mM} \mathrm{NaCl,}, 50 \mathrm{mM}$ Tris $\mathrm{pH}$ 7.4, 2 mM EDTA and 1\% Nonidet P-40. For native gel electrophoresis, 2x Tris-Glycin buffer was added and $20 \mu \mathrm{l}$ of sample were resolved by $10 \%$ Novex Tris-Glycin gel (Life Technologies). For SDS PAGE, 4x Laemmli buffer was added, the sample was heated to $95^{\circ} \mathrm{C}$ for $5 \mathrm{~min}$ and $15 \mu \mathrm{l}$ of sample were resolved by a $12 \%$ NuPAGE Bis-Tris gel (Invitrogen). Proteins were transferred onto a Immobilon-P polyvinylidene difluoride membrane (Millipore) using a Bio-Rad Criterion Blotter. After the transfer membrane was blocked with $10 \%$ nonfat milk in PBS containing 0.05\% Tween 20 for 30 min and incubated with affinity-purified anti-DBNL antibodies diluted at 1:1000 at $4{ }^{\circ} \mathrm{C}$ overnight, followed by treatment with di- 
luted at 1:10000 peroxidase-labeled goat anti-chicken IgY (A16054, Thermo Fisher). Mouse monoclonal anti-actin (clone C4; MP Biomedicals) and mouse monoclonal anti- $\alpha$-tubulin (clone DM1A, Sigma-Aldrich) antibodies were used in combination with peroxidase-labeled goat anti-mouse IgG (A4416, Sigma-Aldrich). The reactivity was detected with an Amersham ECL Detection Reagent (GE Healthcare Life Science) using an Intas gel imager (Intas Science Imaging Instruments $\mathrm{GmbH}$ ). To analyze the electrophoretic mobility of recombinant proteins, $2 \mu \mathrm{M}$ solution of each protein in native sample buffer or in Laemmli buffer were prepared. For native gel electrophoresis, $15 \mu \mathrm{l}$ of the sample were resolved by $10 \%$ Novex Tris-Glycin gel (Life Technologies). For SDS PAGE, the sample was heated to $95{ }^{\circ} \mathrm{C}$ for $5 \mathrm{~min}$ and $15 \mu \mathrm{l}$ of the sample were resolved by $12 \%$ NuPAGE Bis-Tris gel (Invitrogen). Molecular weight markers were purchased from ThermoFisher Scientific (PageRuler ${ }^{T M}$ Plus Prestained Protein Ladder) and Serva (Native Marker 39219). For detection of native markers, a PVDF membrane was stained with Ponceau S (Sigma-Aldrich). Gels were stained with Roti ${ }^{\circledR}$-Blue quick (Roth).

\section{Instrumentation and experimental procedures for FCCS, 2fFCS, FCS and FLIM-FRET imaging}

For FCS, 2fFCS and FCCS experiments, we used the commercial instrument Microtime 200 (PicoQuant GmbH). ${ }^{1}$

$\boldsymbol{F C S}$ : Excitation was done with a linearly polarized pulsed diode laser $(\lambda=485 \mathrm{~nm}$, pulse duration 50 ps FWHM, LDH-P-C-485B, PicoQuant) equipped with a clean-up filter (Brightline FF01-480/17, Semrock). Light of this laser is pulsed at a repetition rate of $40 \mathrm{MHz}$ with a multi-channel picosecond laser driver (PDL 828, "Sepia II", PicoQuant). The laser beam is coupled into a polarization-maintaining single-mode fiber (PMC-4004.2-NA010-3-APC-250V, Schäfter and Kirchhoff GmbH). At the fiber output, the light is collimated and reflected by a dichroic mirror (FITC/TRITC Chroma Technology) into the objective lens of the microscope (UPLSAPO 60x water, 1.2 N.A., Olympus). The same 
water-immersion objective is used to collect fluorescence from the sample. A long-pass filter (BLP01-488R-25, Semrock) is used to block back-scattered light from the laser. The emission light is focused into a pinhole of $100 \mu \mathrm{m}$ diameter, collimated again, and split by a non-polarizing beam splitter cube (Linos Photonics GmbH \& Co. KG) and refocused onto two single-photon avalanche diodes (SPCM-CD 3516 H, Excelitas Technologies GmbH \& Co. KG). A multichannel picosecond event timer (HydraHarp 400, PicoQuant) records the detected photons from both detectors independently with an absolute temporal resolution of 16 ps on a common time frame. For FCS measurements, his-EmGFP-DBNL(1-179), hisEmGFP-DBNL(1-256) and his-EmGFP-DBNL(1-374) were diluted to $10 \mathrm{nM}$ and $100 \mathrm{pM}$ concentration in PBS (D8537, Sigma-Aldrich). The experiments were done by putting $30 \mu \mathrm{l}$ of sample on top of a cleaned glass cover-slip, and during measurements the objective was focused approximately $30 \mu \mathrm{m}$ into the solution.

$\boldsymbol{F C C S}$ : The excitation unit consists of two pulsed diode lasers, blue $(\lambda=485 \mathrm{~nm}, \mathrm{LDH}-\mathrm{P}-$ C-485B, PicoQuant) and red ( $\lambda$ exc $=640$ nm, LDH-D-C 640, PicoQuant), for his-EmGFPDBNL and his-mCherry-DBNL, respectively. Laser pulse width was 50 ps FWHM, and pulse repetition rate was $40 \mathrm{MHz}$. Laser pulsing, beam coupling, and focusing through the objective lens were done as described in the previous section. After the pinhole, the collected emission light was split by a non-polarizing beam splitter cube (Linos Photonics GmbH \& Co. KG) and refocused onto two single-photon avalanche diodes (SPCM-CD 3516 H, Excelitas Technologies GmbH \& Co. KG). Spectral cross-talk between green and red channel was avoided using emission band-pass filter 525/45 (Semrock) and 692/40 (Semrock) in front of each detector. His-EmGFP-DBNL and his-mCherry-DBNL were diluted in PBS (D8537, Sigma-Aldrich) to $1 \mathrm{nM}$ each and $30 \mu \mathrm{l}$ of mixed sample solution was placed on top of a cleaned glass cover-slip for measurement. The same was done for his-EmGFP and his-mCherry. Excitation and detection for 2fFCS were done as described in. ${ }^{2}$

FLIM-FRET Imaging: For FLIM-FRET experiments, we used a home-built confocal setup equipped with an objective lens of high numerical aperture (Apo N, 100× oil, 1.49 NA, 
Olympus Europe, Hamburg, Germany). The excitation unit consists of a pulsed, linearly polarized white light laser (SC400-4-80, Fianium Ltd., pulse width $\sim 50$ ps, repetition rate $80 \mathrm{MHz}$ ) equipped with a tunable filter (AOTFnC 400.650-TN, Pegasus Optik GmbH). For our experiments, we used $488 \mathrm{~nm}$ wavelength for exciting EGFP tagged to actin filaments. Light was reflected using a non-polarizing beam splitter towards the objective lens, and the back-scattered excitation light was blocked with long-pass filters (EdgeBasic BLP01488R, Semrock). A band-pass filter 525/45 (BrightLine FF01-525/45, Semrock) was used for our measurements. Emission light collected by the objective was focused into a pinhole of $100 \mu \mathrm{m}$ diameter, collimated again and refocused into the active area of an avalanche photodiode (Excelitas Technologies Corporation). Data recording was performed with the aid of a multichannel picosecond event timer (HydraHarp 400, PicoQuant GmbH). Individual cells were scanned with a focused laser spot using a piezo-nanopositioning stage (P-562.3CD, Physik Instrumente GmbH).

\section{Data evaluation}

Calculation of intensity autocorrelation and cross-correlation curves for FCCS were done as described in ${ }^{3,4}$ using a custom written MATLAB routine. For FCS, fluorescence correlation curves were calculated from intensity fluctuations using the algorithm of. ${ }^{5}$ Finally, data evaluation and fitting for 2fFCS were performed as described in ${ }^{2}$ utilizing again custom written MATLAB routines. Fluorescence lifetime data evaluation was done using MATLAB software package for lifetime fitting as available here - https://www.joerg-enderlein.de/software.

\section{References}

(1) Buschmann, V., Krämer, B., Koberling, F., Macdonald, R., and Rättinger, S. (2009) Quantitative FCS: determination of the confocal volume by FCS and bead scanning with the microtime 200. Application Note PicoQuant GmbH, Berlin 
(2) Dertinger, T., Pacheco, V., von der Hocht, I., Hartmann, R., Gregor, I., and Enderlein, J. (2007) Two-focus fluorescence correlation spectroscopy: A new tool for accurate and absolute diffusion measurements. ChemPhys Chem 8, 433-443.

(3) Schwille, P., Meyer-Almes, F.-J., and Rigler, R. (1997) Dual-color fluorescence crosscorrelation spectroscopy for multicomponent diffusional analysis in solution. Biophysical journal 72, 1878-1886.

(4) Bacia, K., Majoul, I. V., and Schwille, P. (2002) Probing the endocytic pathway in live cells using dual-color fluorescence cross-correlation analysis. Biophysical journal 83, $1184-1193$.

(5) Wahl, M., Gregor, I., Patting, M., and Enderlein, J. (2003) Fast calculation of fluorescence correlation data with asynchronous time-correlated single-photon counting. Optics express 11, 3583-3591. 


\subsection{Loop formation dynamics and diffusion of disor- dered proteins}

In ref. [174], a Brownian dynamics model (based on bead-rod chain dynamics in a thermal bath including hydrodynamic interactions) was developed by Steffen Mühle for a quantitative understanding of the dynamics of intrinsically disordered peptides (IDPs). The core idea was to measure end-to-end contact rates of IDPs using PET-FCS, and to determine their hydrodynamic radius with dual-focus FCS. Using the theoretical framework developed by Steffen Mühle, the experimentally measured rates and radii were translated into molecular properties of the peptides - the persistence length and hydrodynamic radius per amino acid residue. Repeats of glycine and serine residues of different lengths (GS)n were used for this study. My contribution was the 2fFCS measurement of hydrodynamic radii of the GS repeats. The method has been described in details elsewhere [91]. In the end, these measurements helped us to obtain the following polymer parameter values: a persistence length of $l_{p}=5.2 \pm 1.9 \AA$ and a hydrodynamic radius of $a=3.5 \pm 0.7 \AA$ per amino acid. 


\section{Chapter 6}

\section{Conclusion and Outlook}

D

uring my work on this thesis, I have developed a novel optical method which enables the localization of fluorescent molecules along the optical axis of a microscope with subnanometer accuracy. Furthermore, I have utilized several advanced methods in SMFS for the quantitative investigation of fast dynamics in biological molecules that cannot, to our knowledge, be done easily with conventional methods.

In chapter 3 , I have demonstrated how FLCS can be utilized for probing the rapid $(\sim 1 \mu \mathrm{s})$ photophysical transition between two emission states of EGFP that have different fluorescence lifetimes. This study, as published here [38] was the first experimental application of FLCS to study rapid state transitions in a protein. My study shows that FLCS could be used for a broad range of applications where changes in fluorescence lifetime can be directly connected to, for e.g., local density fluctuations inside a polymer matrix, nano-mechanical motion of a resonator in the quantum regime, or variations in electron transfer rates in complex bio-macromolecules. FLCS can find further exciting applications for investigating fast conformational dynamics in biomolecules and polymer chains such as intrinsically disordered proteins (IDPs). As a method complementary to FRET, FLCS can be efficiently utilized for quantifying correlations of a donor's lifetime, which was for the first time reported here [175], where a fast transition between two conformational states was resolved in a dye-protein construct. Another promising application of FLCS can be the investigation of fast molecular dynamics in combination with MIET. In FLCS-MIET, labeling of the target molecule is done with a single dye instead of two as in FRET. This makes FLCS-MIET technically simpler than FRET, and I believe that the method will find a broad range of applications.

In chapter 4, two published reports encompassing the working principle and applications of graphene- and metal-induced energy transfer (GIET and MIET) was provided. During the course of this thesis, I developed GIET as an advanced variant of MIET, where I 
replaced the metal layer of MIET by a single graphene sheet, which enhanced the axial resolution of the method by nearly tenfold. I have shown that it is possible to localize single immobilized molecules with nanometer accuracy, and that one can measure the distance between fluorophores attached to the two different leaflets of a supported lipid bilayer (SLB). In particular, GIET offers an excellent opportunity to investigate structural biophysics and leaflet-specific dynamics in artificial and cellular membranes which are roughly 4-6 $\mathrm{nm}$ thick. In this vein, we have initiated multiple novel research projects where we use GIET as a tool for precise quantification of the diffusion coefficient of lipids and proteins in membranes, axial localization of membrane proteins, or dynamics and fluctuations in membranes.

A fascinating question in membrane biophysics is how to resolve the lateral diffusion of molecules in a leaflet-specific manner. Lipid molecules and various membrane proteins exhibit different mobilities in each leaflet of a SLB. However, with the existing fluorescent spectroscopic techniques, it is not straightforward to quantify the diffusion coefficients in each leaflet separately. In this respect, FLCS in combination with GIET could be a useful tool. While axial localization of fluorescent molecules in each leaflet is done by GIET using fluorescence lifetimes, diffusion of the fluorophores in each leaflet is resolved using FLCS by calculating ACFs in a lifetime-specific manner. In practice, GIET-FLCS measurements are done similar to scanning FCS [176] using a linear scan path. This approach, known as line-scan FCS (lsFCS, line-scan FLCS in our case) [177] was developed to probe diffusion in membranes which is inherently slow in nature resulting in strong photobleaching when classical stationary FCS is used. In contrast, lsFCS continuously scans the sample with the focused laser beam of a confocal laser-scanning microscope (CLSM) thus significantly reducing photobleaching and significantly improving the statistical accuracy of the diffusion measurements. Scanning along a single line instead of scanning a whole area enhances the temporal resolution and thus increases diffusion-measurement accuracy. LsFCS offers several further advantages: it is insensitive to optical saturation artifacts, or to exact vertical laser focus position with respect to the membrane, and above all, it is calibration-free, which means that the size of the scanning focus is directly inferred from the experiment without the need of measuring it independently. Currently, we utilize GIET-lsFLCS for quantifying diffusion coefficients of fluorescently labeled lipids freely diffusing in a SLB. For this purpose, we use fluorescently labeled lipid head groups (Atto655-DPPE) and mix them with unlabeled lipids (DOPC) for preparation of small unilamellar vesicles (SUVs) which form a SLB after deposition on a substrate. Measurements are done by repeatedly scanning along a linear path, thus obtaining higher photon statistics and accuracy in determining diffusion coefficients. Next, lifetime-specific spatio-temporal ACFs and cross-correlation functions 
(CCFs) are calculated for varying lag space and lag times values. The resulting twodimensional correlograms are then fitted with a mathematical model for obtaining the diffusion coefficients of the fluorophores. Experiments in this direction are currently ongoing.

Another exciting ongoing application of GIET is mapping the dynamics in mitochondrial membranes during ATP synthesis. Mitochondria, popularly known as 'powerhouses of the cell,' are membrane-bound cell organelles which generate most of the chemical energy required for cellular biochemical processes. The energy is produced in the form of adenosine tri-phosphate (ATP). A mitochondrion is constituted by an outer mitochondrial membrane, an inner membrane, the inter-membrane space between outer and inner membranes, the cristae formed by invaginations of the inner membrane, and the matrix between inner membranes. The outer membrane is very similar to a cell membrane and contains integral membrane proteins called porins. The inner membrane is mostly involved in various functions of a mitochondrion such as electron transport chain (ETC) redox reactions and the synthesis of ATP in the matrix via the enzyme ATP synthase. It also contains transport proteins that govern the passage of metabolites in and out of the matrix. Our goal is to map the mitochondrial membranes and their dynamics using GIET. For this purpose, we label the inner membrane of live isolated mitochondria with the mitochondria-specific fluorophore mitotracker deep red and acquire FLIM images. To differentiate between pre- and post-ATP-synthesizing states, we record FLIM images of the mitochondria on a graphene substrate in the 'resting' state, followed by activation with a mixture of pyruvate, malate, and ADP, and then repeat the FLIM imaging in the 'activated' state. The core idea is to generate height maps of the inner membrane in the 'resting' and 'activated' states, and to observe whether there exist dynamic height fluctuations of the membrane between these two states.

Not only the fluorescence lifetime varies monotonously with distance from a MIET or GIET substrate, but also the fluorescence intensity or brightness. This modulation of the fluorescence intensity due to quenching by the metal or graphene can be utilized for following conformational fluctuations in biomolecules with nanoscond temporal resolution. For this purpose, we combine scanning-FCS and MIET/GIET, and then scan immobilized molecules tagged with a single dye to extract height fluctuations of the dye, which reflects the underlying conformational dynamics of the tagged molecule. As the proof-ofconcept, we currently implement this method to study the dynamics of DNA hairpins. We call this technique dynamic MIET or dynaMIET. Briefly, we use a DNA hairpin loop with its 5' end labeled with Atto 647N. The loop is attached to a dsDNA stalk which itself is attached to a DNA origami platform. This custom-designed origami platform is functionally immobilized on a passivated silicon dioxide spacer above a thin gold film. The position of the fluorophore with respect to the gold layer changes due to the hairpin 
loop's opening and closing dynamics, leading to characteristic fluorescence intensity fluctuations that can be measured with FCS. With dynaMIET, we hope to simultaneously probe the two-state dynamics of the hairpin loop which takes place on a timescale of $\sim 1 \mathrm{~ms}$, but also the fast vertical diffusion of the DNA chain in its 'opened' state which takes place on timescales of hundreds of nanoseconds. Until now, sm-FRET remains the technique of choice for probing such chain dynamics. Unlike sm-FRET, dynaMIET requires only a single fluorescent tag, and it has a working distance of $\sim 150 \mathrm{~nm}$, so that it can be efficiently utilized for probing long chain dynamics on any timescale slower than the fluorescence lifetime of the dye. I believe that dynaMIET will find many applications especially for probing chain dynamics of intrinsically disordered proteins (IDPs), nucleic acids, or other polymers.

Three-dimensional localization of single molecules is a major goal of SMLM-based techniques. MIET or GIET, in combination with existing SMLM methods, can offer a versatile and robust alternative for three-dimensional localization of single fluorescent molecules. In this direction, we have recently combined FLIM with two SMLM techniques, dSTORM and DNA-PAINT, using a CLSM equipped with a pulsed laser, single-photon counting detectors, and TCSPC electronics [178]. In this way, we combined the multiple advantages of CLSM with those of SMLM. In particular, CLSM provides single-photon sensitivity, axial sectioning, pixel-free imaging, and fluorescence lifetime information, whereas SMLM provides lateral super-resolution. This approach of fluorescence lifetime-SMLM (FL-SMLM) has been demonstrated by imaging fluorescently labeled microtubules and clathrins in COS7 cells. The next development will be to combine FL-SMLM with MIET for axial localization, thus realizing dSTORM-MIET or DNA-PAINT-MIET as an approach to three-dimensional super-resolution microscopy. However, one major drawback of CLSM-TCSPC for FLIM imaging is that it is three to five times slower than widefield microscopy. For MIET experiments, we recently obtained a new FLIM camera (LINCam25, Photonscore GmbH) and could successfully use it for fluorescence lifetime imaging of single-molecule molecules [179]. Using this camera, we could successfully localize and distinguish different single molecules (Cy5, Atto 655, and Atto 647N) with similar emission spectra but distinct fluorescence lifetimes. The next step will be to combine this with MIET/GIET for realizing three-dimensional wide-field SMLM.

Finally yet importantly, the next goal in MIET and GIET imaging is to utilize fluorescent proteins as probes instead of organic dyes. This will unlock the huge potential of the technique for live-cell imaging. In this direction, we currently perform MIET measurements of the nanometric undulations of an active cell membrane. For this purpose, we utilize Dictyostelium discoideum (D.d.), a social amoeba growing in soil and which is frequently used as a model organism for investigating cell adhesion, motility, chemotaxis, and signal transduction [180]. We tagged the surface cyclic AMP receptor 1 (cAR1) with 
a GFP variant. Binding of cyclic AMP (cAMP) to cAR1 results in an amplification of actin polymerization at the leading edge of D.d., resulting on the formation of membrane protrusions known as pseudopodia.

Chapter 5 illustrated the application of various single-molecule spectroscopic and imaging techniques for elucidating the structure-function relationship and fast dynamics in proteins. I utilized classical FCS, dual-focus FCS, FCCS, PET-FCS, and FLIM-FRET imaging for studying the dimerization and oligomerization of human DBNL, and for investigating the conformational dynamics of intrinsically disordered GS repeat peptides. These studies are superb examples of the capabilities of single-molecule spectroscopy for investigating biomolecules with heterogeneous structure and dynamics at the singlemolecule level. 


\section{Bibliography}

[1] Osamu Shimomura, Frank H Johnson, and Yo Saiga. Extraction, purification and properties of aequorin, a bioluminescent protein from the luminous hydromedusan, aequorea. Journal of cellular and comparative physiology, 59(3):223-239, 1962.

[2] Douglas C Prasher, Virginia K Eckenrode, William W Ward, Frank G Prendergast, Milton J Cormier, et al. Primary structure of the aequorea victoria green-fluorescent protein. Gene, 111(2):229-233, 1992.

[3] Stefan W Hell and Jan Wichmann. Breaking the diffraction resolution limit by stimulated emission: stimulated-emission-depletion fluorescence microscopy. Optics letters, 19(11):780-782, 1994.

[4] William E Moerner and Lothar Kador. Optical detection and spectroscopy of single molecules in a solid. Physical review letters, 62(21):2535, 1989.

[5] M Orrit and J Bernard. Single molecule spectroscopy in a solid. Journal of luminescence, 53(1-6):165-169, 1992.

[6] E Brooks Shera, Newton K Seitzinger, Lloyd M Davis, Richard A Keller, and Steven A Soper. Detection of single fluorescent molecules. Chemical Physics Letters, 174(6):553-557, 1990.

[7] Eric Betzig, George H Patterson, Rachid Sougrat, O Wolf Lindwasser, Scott Olenych, Juan S Bonifacino, Michael W Davidson, Jennifer Lippincott-Schwartz, and Harald F Hess. Imaging intracellular fluorescent proteins at nanometer resolution. Science, 313(5793):1642-1645, 2006.

[8] Michael J Rust, Mark Bates, and Xiaowei Zhuang. Sub-diffraction-limit imaging by stochastic optical reconstruction microscopy (storm). Nature methods, 3(10): 793-796, 2006. 
[9] Mike Heilemann, Sebastian Van De Linde, Mark Schüttpelz, Robert Kasper, Britta Seefeldt, Anindita Mukherjee, Philip Tinnefeld, and Markus Sauer. Subdiffractionresolution fluorescence imaging with conventional fluorescent probes. Angewandte Chemie International Edition, 47(33):6172-6176, 2008.

[10] Alexey Sharonov and Robin M Hochstrasser. Wide-field subdiffraction imaging by accumulated binding of diffusing probes. Proceedings of the National Academy of Sciences, 103(50):18911-18916, 2006.

[11] Max Knoll and Ernst Ruska. Das elektronenmikroskop. Zeitschrift für physik, 78 (5-6):318-339, 1932.

[12] Manfred von Ardenne. On the history of scanning electron microscopy, of the electron microprobe, and of early contributions to transmission electron microscopy. In "The Beginnings of Electron Microscopy", pages 1-21. Academic Press Orlando, 1985.

[13] Lawrence Bragg. X-ray crystallography. Scientific American, 219(1):58-74, 1968.

[14] Jacques Dubochet. Cryo-em - the first thirty years. Journal of microscopy, 245(3): 221-224, 2012.

[15] Bo Huang, Wenqin Wang, Mark Bates, and Xiaowei Zhuang. Three-dimensional super-resolution imaging by stochastic optical reconstruction microscopy. Science, 319(5864):810-813, 2008.

[16] Manuel F Juette, Travis J Gould, Mark D Lessard, Michael J Mlodzianoski, Bhupendra S Nagpure, Brian T Bennett, Samuel T Hess, and Joerg Bewersdorf. Threedimensional sub-100 nm resolution fluorescence microscopy of thick samples. Nature methods, 5(6):527-529, 2008.

[17] Mikael P Backlund, Matthew D Lew, Adam S Backer, Steffen J Sahl, Ginni Grover, Anurag Agrawal, Rafael Piestun, and WE Moerner. Simultaneous, accurate measurement of the $3 \mathrm{~d}$ position and orientation of single molecules. Proceedings of the National Academy of Sciences, 109(47):19087-19092, 2012.

[18] Gleb Shtengel, James A Galbraith, Catherine G Galbraith, Jennifer LippincottSchwartz, Jennifer M Gillette, Suliana Manley, Rachid Sougrat, Clare M Waterman, Pakorn Kanchanawong, Michael W Davidson, et al. Interferometric fluorescent super-resolution microscopy resolves 3d cellular ultrastructure. Proceedings of the National Academy of Sciences, 106(9):3125-3130, 2009. 
[19] Daniel Aquino, Andreas Schönle, Claudia Geisler, Claas v Middendorff, Christian A Wurm, Yosuke Okamura, Thorsten Lang, Stefan W Hell, and Alexander Egner. Two-color nanoscopy of three-dimensional volumes by 4pi detection of stochastically switched fluorophores. Nature methods, 8(4):353-359, 2011.

[20] Roman Schmidt, Christian A Wurm, Stefan Jakobs, Johann Engelhardt, Alexander Egner, and Stefan W Hell. Spherical nanosized focal spot unravels the interior of cells. Nature methods, 5(6):539-544, 2008.

[21] Stefan W Hell, Roman Schmidt, and Alexander Egner. Diffraction-unlimited threedimensional optical nanoscopy with opposing lenses. Nature Photonics, 3(7):381$387,2009$.

[22] Alexey I Chizhik, Jan Rother, Ingo Gregor, Andreas Janshoff, and Jörg Enderlein. Metal-induced energy transfer for live cell nanoscopy. Nature Photonics, 8(2):124$127,2014$.

[23] KH Drexhage. Influence of a dielectric interface on fluorescence decay time. Journal of luminescence, 1:693-701, 1970.

[24] Arindam Ghosh, Akshita Sharma, Alexey I Chizhik, Sebastian Isbaner, Daja Ruhlandt, Roman Tsukanov, Ingo Gregor, Narain Karedla, and Jörg Enderlein. Graphene-based metal-induced energy transfer for sub-nanometre optical localization. Nature Photonics, 13(12):860-865, 2019.

[25] Shimon Weiss. Fluorescence spectroscopy of single biomolecules. Science, 283 (5408):1676-1683, 1999.

[26] Rahul Roy, Sungchul Hohng, and Taekjip Ha. A practical guide to single-molecule fret. Nature methods, 5(6):507-516, 2008.

[27] Nam Ki Lee, Hye Ran Koh, Kyu Young Han, Jihyun Lee, and Seong Keun Kim. Single-molecule, real-time measurement of enzyme kinetics by alternating-laser excitation fluorescence resonance energy transfer. Chemical communications, 46(26): 4683-4685, 2010.

[28] Benjamin Schuler and William A Eaton. Protein folding studied by single-molecule fret. Current opinion in structural biology, 18(1):16-26, 2008.

[29] Everett A Lipman, Benjamin Schuler, Olgica Bakajin, and William A Eaton. Singlemolecule measurement of protein folding kinetics. Science, 301(5637):1233-1235, 2003. 
[30] Elliot L Elson and Douglas Magde. Fluorescence correlation spectroscopy. i. conceptual basis and theory. Biopolymers: Original Research on Biomolecules, 13(1): $1-27,1974$.

[31] Markus Sauer and Hannes Neuweiler. Pet-fcs: probing rapid structural fluctuations of proteins and nucleic acids by single-molecule fluorescence quenching. In Fluorescence Spectroscopy and Microscopy, pages 597-615. Springer, 2014.

[32] Lewis E Kay. Nmr studies of protein structure and dynamics. Journal of Magnetic Resonance, 213(2):477-491, 2011.

[33] Horst Kessler, Matthias Gehrke, and Christian Griesinger. Two-dimensional nmr spectroscopy: Background and overview of the experiments [new analytical methods (36)]. Angewandte Chemie International Edition in English, 27(4):490-536, 1988.

[34] Thomas Prisner, Martin Rohrer, and Fraser MacMillan. Pulsed epr spectroscopy: biological applications. Annual review of physical chemistry, 52(1):279-313, 2001.

[35] Pau Bernadó, Efstratios Mylonas, Maxim V Petoukhov, Martin Blackledge, and Dmitri I Svergun. Structural characterization of flexible proteins using small-angle x-ray scattering. Journal of the American Chemical Society, 129(17):5656-5664, 2007.

[36] B Jacrot. The study of biological structures by neutron scattering from solution. Reports on progress in physics, 39(10):911, 1976.

[37] Martin Böhmer, Michael Wahl, Hans-Jürgen Rahn, Rainer Erdmann, and Jörg Enderlein. Time-resolved fluorescence correlation spectroscopy. Chemical Physics Letters, 353(5-6):439-445, 2002.

[38] Arindam Ghosh, Sebastian Isbaner, Manoel Veiga-Gutiérrez, Ingo Gregor, Jörg Enderlein, and Narain Karedla. Quantifying microsecond transition times using fluorescence lifetime correlation spectroscopy. The journal of physical chemistry letters, 8(24):6022-6028, 2017.

[39] Bernard Valeur. Molecular fluorescence. Digital Encyclopedia of Applied Physics, pages $477-531,2003$.

[40] Sandra Goutte, Matthew J Mason, Marta M Antoniazzi, Carlos Jared, Didier Merle, Lilian Cazes, Luís Felipe Toledo, Hanane El-Hafci, Stéphane Pallu, Hugues Portier, et al. Intense bone fluorescence reveals hidden patterns in pumpkin toadlets. Scientific reports, 9(1):1-8, 2019. 
[41] FWJ Teale and Go Weber. Ultraviolet fluorescence of the aromatic amino acids. Biochemical Journal, 65(3):476-482, 1957.

[42] Markus Lippitz, Wolfgang Erker, Heinz Decker, KE Van Holde, and Thomas Basche. Two-photon excitation microscopy of tryptophan-containing proteins. Proceedings of the National Academy of Sciences, 99(5):2772-2777, 2002.

[43] Mark Schüttpelz, Christian Müller, Hannes Neuweiler, and Markus Sauer. Uv fluorescence lifetime imaging microscopy: A label-free method for detection and quantification of protein interactions. Analytical chemistry, 78(3):663-669, 2006.

[44] PA Liebman and RA Leigh. Autofluorescence of visual receptors. Nature, 221 (5187):1249-1251, 1969.

[45] Otto Warburg and Walter Christian. On a new oxidation enzyme and its absorption spectrum. Biochem Z, 254:438-458, 1932.

[46] DANIEL Wellner. Flavoproteins. Annual review of biochemistry, 36(1):669-690, 1967.

[47] Roger Y Tsien. The green fluorescent protein. Annual review of biochemistry, 67 (1):509-544, 1998.

[48] Yan Chen, Joachim D Müller, QiaoQiao Ruan, and Enrico Gratton. Molecular brightness characterization of egfp in vivo by fluorescence fluctuation spectroscopy. Biophysical journal, 82(1):133-144, 2002.

[49] Jingdong Zhu, Mary Lynn Musco, and Michael J Grace. Three-color flow cytometry analysis of tricistronic expression of ebfp, egfp, and eyfp using emcv-ires linkages. Cytometry: The Journal of the International Society for Analytical Cytology, 37(1): 51-59, 1999.

[50] Mickaël Lelimousin, Marjolaine Noirclerc-Savoye, Christelle Lazareno-Saez, Bernhard Paetzold, Sophie Le Vot, Richard Chazal, Pauline Macheboeuf, Martin J Field, Dominique Bourgeois, and Antoine Royant. Intrinsic dynamics in ecfp and cerulean control fluorescence quantum yield. Biochemistry, 48(42):10038-10046, 2009.

[51] Antoine Royant and Marjolaine Noirclerc-Savoye. Stabilizing role of glutamic acid 222 in the structure of enhanced green fluorescent protein. Journal of structural biology, 174(2):385-390, 2011.

[52] M Sauer, KT Han, R Müller, A Schulz, R Tadday, S Seeger, J Wolfrum, J ArdenJacob, G Deltau, NJ Marx, et al. New fluorescent labels for time-resolved detection of biomolecules. Journal of fluorescence, 3(3):131-139, 1993. 
[53] M Sauer, K-T Han, R Müller, S Nord, A Schulz, S Seeger, J Wolfrum, J ArdenJacob, G Deltau, NJ Marx, et al. New fluorescent dyes in the red region for biodiagnostics. Journal of fluorescence, 5(3):247-261, 1995.

[54] Lauren A Ernst, Ravinder K Gupta, Ratnakar B Mujumdar, and Alan S Waggoner. Cyanine dye labeling reagents for sulfhydryl groups. Cytometry: The Journal of the International Society for Analytical Cytology, 10(1):3-10, 1989.

[55] I Hemmilä. Fluorescent labels for use in fluorescence immunoassay. Appl. Fluor. Technol., 1:1-8, 1989.

[56] James E Whitaker, Rosaria P Haugland, Diane Ryan, Peter C Hewitt, Richard P Haugland, and Franklyn G Prendergast. Fluorescent rhodol derivatives: versatile, photostable labels and tracers. Analytical biochemistry, 207(2):267-279, 1992.

[57] Jyh Myng Zen and Gabor Patonay. Near-infrared fluorescence probe for ph determination. Analytical Chemistry, 63(24):2934-2938, 1991.

[58] David A Zacharias, Jonathan D Violin, Alexandra C Newton, and Roger Y Tsien. Partitioning of lipid-modified monomeric gfps into membrane microdomains of live cells. Science, 296(5569):913-916, 2002.

[59] Greg T Hermanson. Bioconjugate techniques. Academic press, 2013.

[60] MD Partis, DG Griffiths, GC Roberts, and RB Beechey. Cross-linking of protein by $\omega$-maleimido alkanoyln-hydroxysuccinimido esters. Journal of Protein Chemistry, $2(3): 263-277,1983$.

[61] Chung-Wein Lee, Yan J Ren, Mathieu Marella, Maria Wang, James Hartke, and Suzana S Couto. Multiplex immunofluorescence staining and image analysis assay for diffuse large b cell lymphoma. Journal of Immunological Methods, 478:112714, 2020.

[62] Sergio T Ferreira, Lorenzo Stella, and Enrico Gratton. Conformational dynamics of bovine $\mathrm{cu}$, $\mathrm{zn}$ superoxide dismutase revealed by time-resolved fluorescence spectroscopy of the single tyrosine residue. Biophysical journal, 66(4):1185-1196, 1994.

[63] William R Ware, Sai K Lee, Gary J Brant, and Peter P Chow. Nanosecond timeresolved emission spectroscopy: spectral shifts due to solvent-excited solute relaxation. The Journal of Chemical Physics, 54(11):4729-4737, 1971. 
[64] ASR Koti, MMG Krishna, and N Periasamy. Time-resolved area-normalized emission spectroscopy (tranes): a novel method for confirming emission from two excited states. The Journal of Physical Chemistry A, 105(10):1767-1771, 2001.

[65] Richard KP Benninger, Oliver Hofmann, James McGinty, Jose Requejo-Isidro, Ian Munro, Mark AA Neil, Andrew J Demello, and Paul MW French. Time-resolved fluorescence imaging of solvent interactions in microfluidic devices. Optics express, 13(16):6275-6285, 2005.

[66] David P Millar. Time-resolved fluorescence spectroscopy. Current opinion in structural biology, 6(5):637-642, 1996.

[67] Eric Betzig, Jay K Trautman, TD Harris, JS Weiner, and RL Kostelak. Breaking the diffraction barrier: optical microscopy on a nanometric scale. Science, 251 (5000):1468-1470, 1991.

[68] Gerhard J Schütz, Hansgeorg Schindler, and Thomas Schmidt. Single-molecule microscopy on model membranes reveals anomalous diffusion. Biophysical journal, $73(2): 1073,1997$.

[69] Thomas Schmidt, GJ Schütz, W Baumgartner, HJ Gruber, and H Schindler. Imaging of single molecule diffusion. Proceedings of the National Academy of Sciences, 93(7):2926-2929, 1996.

[70] Peter Hinterdorfer, Gerhard Schütz, Ferry Kienberger, and Hansgeorg Schindler. Detection and characterization of single biomolecules at surfaces. Reviews in Molecular Biotechnology, 82(1):25-35, 2001.

[71] Werner Trabesinger, Gerhard J Schütz, Herrmann J Gruber, Hansgeorg Schindler, and Thomas Schmidt. Detection of individual oligonucleotide pairing by singlemolecule microscopy. Analytical chemistry, 71(1):279-283, 1999.

[72] Hsuan-Lei Sung and David J Nesbitt. Single-molecule fret kinetics of the mn2+ riboswitch: evidence for allosteric mg2+ control of "induced-fit" vs "conformational selection" folding pathways. The Journal of Physical Chemistry B, 123(9):20052015, 2019.

[73] Rula Masoud, Roman Tsukanov, Toma E Tomov, Noa Plavner, Miran Liber, and Eyal Nir. Studying the structural dynamics of bipedal dna motors with singlemolecule fluorescence spectroscopy. ACS nano, 6(7):6272-6283, 2012.

[74] Igor L Medintz and Niko Hildebrandt. FRET-Förster resonance energy transfer: from theory to applications. John Wiley \& Sons, 2013. 
[75] Sharonda J LeBlanc, Prakash Kulkarni, and Keith R Weninger. Single molecule fret: A powerful tool to study intrinsically disordered proteins. Biomolecules, 8(4): $140,2018$.

[76] Lauren Ann Metskas and Elizabeth Rhoades. Single-molecule fret of intrinsically disordered proteins. Annual Review of Physical Chemistry, 71:391-414, 2020.

[77] Dilip Shrestha, Attila Jenei, Péter Nagy, György Vereb, and János Szöllősi. Understanding fret as a research tool for cellular studies. International journal of molecular sciences, 16(4):6718-6756, 2015.

[78] Thomas Heinlein, Jens-Peter Knemeyer, Oliver Piestert, and Markus Sauer. Photoinduced electron transfer between fluorescent dyes and guanosine residues in dnahairpins. The Journal of Physical Chemistry B, 107(31):7957-7964, 2003.

[79] Douglas Magde, Elliot L Elson, and Watt W Webb. Fluorescence correlation spectroscopy. ii. an experimental realization. Biopolymers: Original Research on Biomolecules, 13(1):29-61, 1974.

[80] Manfred Eigen and Rudolf Rigler. Sorting single molecules: application to diagnostics and evolutionary biotechnology. Proceedings of the National Academy of Sciences, 91(13):5740-5747, 1994.

[81] Stephanie Bleicken, Miki Otsuki, and Ana J Garcia-Saez. Quantification of proteinprotein interactions within membranes by fluorescence correlation spectroscopy. Current Protein and Peptide Science, 12(8):691-698, 2011.

[82] Tedman Torres and Marcia Levitus. Measuring conformational dynamics: a new fcs-fret approach. The Journal of Physical Chemistry B, 111(25):7392-7400, 2007.

[83] Jerker Widengren and Petra Schwille. Characterization of photoinduced isomerization and back-isomerization of the cyanine dye cy5 by fluorescence correlation spectroscopy. The Journal of Physical Chemistry A, 104(27):6416-6428, 2000.

[84] NO Petersen and EL Elson. [19] measurements of diffusion and chemical kinetics by fluorescence photobleaching recovery and fluorescence correlation spectroscopy. In Methods in enzymology, volume 130, pages 454-484. Elsevier, 1986.

[85] Jerker Widengren and Ülo Mets. Conceptual basis of fluorescence correlation spectroscopy and related techniques as tools in bioscience. Single Molecule Detection in Solution: Methods and Applications, pages 69-120, 2002.

[86] Rudolf Rigler and Elliot S Elson. Fluorescence correlation spectroscopy: theory and applications, volume 65. Springer Science \& Business Media, 2012. 
[87] Petra Schwille. Fluorescence correlation spectroscopy and its potential for intracellular applications. Cell biochemistry and biophysics, 34(3):383-408, 2001.

[88] Joachim D Müller, Yan Chen, and Enrico Gratton. [4] fluorescence correlation spectroscopy. In Methods in enzymology, volume 361, pages 69-92. Elsevier, 2003.

[89] Nancy L Thompson, Alena M Lieto, and Noah W Allen. Recent advances in fluorescence correlation spectroscopy. Current opinion in structural biology, 12(5): 634-641, 2002.

[90] Oleg Krichevsky and Grégoire Bonnet. Fluorescence correlation spectroscopy: the technique and its applications. Reports on Progress in Physics, 65(2):251, 2002.

[91] Thomas Dertinger, Victor Pacheco, Iris von der Hocht, Rudolf Hartmann, Ingo Gregor, and Jörg Enderlein. Two-focus fluorescence correlation spectroscopy: A new tool for accurate and absolute diffusion measurements. ChemPhysChem, 8(3): 433-443, 2007.

[92] Thomas Dertinger, Anastasia Loman, Benjamin Ewers, Claus B Müller, Benedikt Krämer, and Jörg Enderlein. The optics and performance of dual-focus fluorescence correlation spectroscopy. Optics express, 16(19):14353-14368, 2008.

[93] Kerstin Weiß, Andreas Neef, Qui Van, Stefanie Kramer, Ingo Gregor, and Jörg Enderlein. Quantifying the diffusion of membrane proteins and peptides in black lipid membranes with 2-focus fluorescence correlation spectroscopy. Biophysical journal, 105(2):455-462, 2013.

[94] Kerstin Weiß and Jörg Enderlein. Lipid diffusion within black lipid membranes measured with dual-focus fluorescence correlation spectroscopy. ChemPhysChem, 13(4):990-1000, 2012.

[95] Petra Schwille, Franz-Josef Meyer-Almes, and Rudolf Rigler. Dual-color fluorescence cross-correlation spectroscopy for multicomponent diffusional analysis in solution. Biophysical journal, 72(4):1878-1886, 1997.

[96] Kirsten Bacia, Irina V Majoul, and Petra Schwille. Probing the endocytic pathway in live cells using dual-color fluorescence cross-correlation analysis. Biophysical journal, 83(2):1184-1193, 2002.

[97] Kirsten Bacia and Petra Schwille. A dynamic view of cellular processes by in vivo fluorescence auto-and cross-correlation spectroscopy. Methods, 29(1):74-85, 2003. 
[98] Aleš Benda, Martin Hof, Michael Wahl, Matthias Patting, Rainer Erdmann, and Peter Kapusta. Tcspc upgrade of a confocal fcs microscope. Review of scientific instruments, 76(3):033106, 2005.

[99] Desmond O'Connor. Time-correlated single photon counting. Academic Press, 2012.

[100] Enrico Gratton, David M Jameson, and Robert D Hall. Multifrequency phase and modulation fluorometry. Annual review of biophysics and bioengineering, 13(1): 105-124, 1984.

[101] Michael Wahl. Modern tcspc electronics: principles and acquisition modes. In Advanced Photon Counting, pages 1-21. Springer, 2014.

[102] Arindam Ghosh, Narain Karedla, Jan Christoph Thiele, Ingo Gregor, and Jörg Enderlein. Fluorescence lifetime correlation spectroscopy: Basics and applications. Methods, 140:32-39, 2018.

[103] Jörg Enderlein and Ingo Gregor. Using fluorescence lifetime for discriminating detector afterpulsing in fluorescence-correlation spectroscopy. Review of Scientific Instruments, 76(3):033102, 2005.

[104] Jana Humpolíčková, Lenka Beranová, Miroslav Štěpánek, Aleš Benda, Karel Procházka, and Martin Hof. Fluorescence lifetime correlation spectroscopy reveals compaction mechanism of 10 and $49 \mathrm{kbp}$ dna and differences between polycation and cationic surfactant. The Journal of Physical Chemistry B, 112(51):16823-16829, 2008 .

[105] Jose M Paredes, Luis Crovetto, Angel Orte, Jose M Alvarez-Pez, and Eva M Talavera. Influence of the solvent on the ground-and excited-state buffer-mediated proton-transfer reactions of a xanthenic dye. Physical Chemistry Chemical Physics, 13(4):1685-1694, 2011.

[106] Giuseppe Vicidomini, Haisen Ta, Alf Honigmann, Veronika Mueller, Mathias P Clausen, Dominic Waithe, Silvia Galiani, Erdinc Sezgin, Alberto Diaspro, Stefan W Hell, et al. Sted-flcs: an advanced tool to reveal spatiotemporal heterogeneity of molecular membrane dynamics. Nano letters, 15(9):5912-5918, 2015.

[107] Ernst Abbe. Beiträge zur theorie des mikroskops und der mikroskopischen wahrnehmung. Archiv für mikroskopische Anatomie, 9(1):413-468, 1873.

[108] Jacques M Vasse and Georges L Truchet. The rhizobium-legume symbiosis: observation of root infection by bright-field microscopy after staining with methylene blue. Planta, 161(6):487-489, 1984. 
[109] Walter Lang. Nomarski differential interference-contrast microscopy. Carl Zeiss, 1982.

[110] RG Fassett, BernadetteA Horgan, and TH Mathew. Detection of glomerular bleeding by phase-contrast microscopy. The Lancet, 319(8287):1432-1434, 1982.

[111] AV Zelenin. Fluorescence microscopy of lysosomes and related structures in living cells. Nature, 212(5060):425-426, 1966.

[112] GJ Brakenhoff, P Blom, and P Barends. Confocal scanning light microscopy with high aperture immersion lenses. Journal of Microscopy, 117(2):219-232, 1979.

[113] CJR Sheppard, DK Hamilton, and HJ Matthews. Scanning optical microscopy of low-contrast samples. Nature, 334(6183):572-572, 1988.

[114] Alberto Diaspro et al. Confocal and two-photon microscopy: foundations, applications, and advances, volume 1. Wiley-Liss New York:, 2002.

[115] Brian Matsumoto. Cell biological applications of confocal microscopy. Elsevier, 2003.

[116] Erik B van Munster and Theodorus WJ Gadella. Fluorescence lifetime imaging microscopy (flim). In Microscopy techniques, pages 143-175. Springer, 2005.

[117] Ammasi Periasamy and Robert M Clegg. FLIM microscopy in biology and medicine. CRC Press, 2009.

[118] Theodorus WJ Gadella Jr, Thomas M Jovin, and Robert M Clegg. Fluorescence lifetime imaging microscopy (flim): spatial resolution of microstructures on the nanosecond time scale. Biophysical chemistry, 48(2):221-239, 1993.

[119] Bo Zhang, Josiane Zerubia, and Jean-Christophe Olivo-Marin. Gaussian approximations of fluorescence microscope point-spread function models. Applied optics, 46(10):1819-1829, 2007.

[120] Claudio Rivolta. Airy disk diffraction pattern: comparison of some values of f/no. and obscuration ratio. Applied optics, 25(14):2404-2408, 1986.

[121] Mats GL Gustafsson. Surpassing the lateral resolution limit by a factor of two using structured illumination microscopy. Journal of microscopy, 198(2):82-87, 2000.

[122] Claus B Müller and Jörg Enderlein. Image scanning microscopy. Physical review letters, 104(19):198101, 2010. 
[123] Ingo Gregor, Martin Spiecker, Roman Petrovsky, Jörg Großhans, Robert Ros, and Jörg Enderlein. Rapid nonlinear image scanning microscopy. Nature methods, 14 (11):1087, 2017.

[124] Volker Westphal, Silvio O Rizzoli, Marcel A Lauterbach, Dirk Kamin, Reinhard Jahn, and Stefan W Hell. Video-rate far-field optical nanoscopy dissects synaptic vesicle movement. Science, 320(5873):246-249, 2008.

[125] Hans Blom and Jerker Widengren. Sted microscopy - towards broadened use and scope of applications. Current opinion in chemical biology, 20:127-133, 2014.

[126] Janina Hanne, Henning J Falk, Frederik Görlitz, Patrick Hoyer, Johann Engelhardt, Steffen J Sahl, and Stefan W Hell. Sted nanoscopy with fluorescent quantum dots. Nature communications, 6(1):1-6, 2015.

[127] Christian Eggeling, Katrin I Willig, and Francisco J Barrantes. Sted microscopy of living cells-new frontiers in membrane and neurobiology. Journal of Neurochemistry, 126(2):203-212, 2013.

[128] Francisco Balzarotti, Yvan Eilers, Klaus C Gwosch, Arvid H Gynnå, Volker Westphal, Fernando D Stefani, Johan Elf, and Stefan W Hell. Nanometer resolution imaging and tracking of fluorescent molecules with minimal photon fluxes. Science, 355(6325):606-612, 2017.

[129] Klaus C Gwosch, Jasmin K Pape, Francisco Balzarotti, Philipp Hoess, Jan Ellenberg, Jonas Ries, and Stefan W Hell. Minflux nanoscopy delivers 3d multicolor nanometer resolution in cells. Nature methods, 17(2):217-224, 2020.

[130] Samuel T Hess, Thanu PK Girirajan, and Michael D Mason. Ultra-high resolution imaging by fluorescence photoactivation localization microscopy. Biophysical journal, 91(11):4258-4272, 2006.

[131] Joerg Schnitzbauer, Maximilian T Strauss, Thomas Schlichthaerle, Florian Schueder, and Ralf Jungmann. Super-resolution microscopy with dna-paint. Nature protocols, 12(6):1198, 2017.

[132] Hari Shroff, Helen White, and Eric Betzig. Photoactivated localization microscopy (palm) of adhesion complexes. Current protocols in cell biology, 41(1):4-21, 2008.

[133] Andrew G York, Alireza Ghitani, Alipasha Vaziri, Michael W Davidson, and Hari Shroff. Confined activation and subdiffractive localization enables whole-cell palm with genetically expressed probes. Nature methods, 8(4):327-333, 2011. 
[134] Sang-Hyuk Lee, Jae Yen Shin, Antony Lee, and Carlos Bustamante. Counting single photoactivatable fluorescent molecules by photoactivated localization microscopy (palm). Proceedings of the National Academy of Sciences, 109(43):17436-17441, 2012.

[135] Suliana Manley, Jennifer M Gillette, and Jennifer Lippincott-Schwartz. Singleparticle tracking photoactivated localization microscopy for mapping singlemolecule dynamics. In Methods in enzymology, volume 475, pages 109-120. Elsevier, 2010.

[136] Ylli Doksani, John Y Wu, Titia de Lange, and Xiaowei Zhuang. Super-resolution fluorescence imaging of telomeres reveals trf2-dependent t-loop formation. Cell, 155 (2):345-356, 2013.

[137] Ke Xu, Guisheng Zhong, and Xiaowei Zhuang. Actin, spectrin, and associated proteins form a periodic cytoskeletal structure in axons. Science, 339(6118):452456, 2013.

[138] Adish Dani, Bo Huang, Joseph Bergan, Catherine Dulac, and Xiaowei Zhuang. Superresolution imaging of chemical synapses in the brain. Neuron, 68(5):843-856, 2010.

[139] Sebastian Van de Linde, Anna Löschberger, Teresa Klein, Meike Heidbreder, Steve Wolter, Mike Heilemann, and Markus Sauer. Direct stochastic optical reconstruction microscopy with standard fluorescent probes. Nature protocols, 6(7):991, 2011.

[140] Teresa Klein, Anna Löschberger, Sven Proppert, Steve Wolter, Sebastian van de Linde, and Markus Sauer. Live-cell dstorm with snap-tag fusion proteins. Nature methods, 8(1):7-9, 2011.

[141] Markus Sauer, Sebastian van de Linde, Thorge Holm, Sarah Aufmkolk, Nadine Ehmann, Robert Blum, Dana Bar-On, Amit Alon, Uri Ashery, Dimitrij Ljaschenko, et al. dstorm of synaptic proteins. Biophysical Journal, 104(2):341a, 2013.

[142] Antonia Goehler, Nadja Bertleff, Malte Timm, Sören Doose, Juergen Seibel, and Markus Sauer. Super-resolution dstorm imaging of human galectin-1 interacting with neuroblastoma cells. Biophysical Journal, 102(3):223a, 2012.

[143] Thomas Nerreter, Sebastian Letschert, Ralph Götz, Sören Doose, Sophia Danhof, Hermann Einsele, Markus Sauer, and Michael Hudecek. Super-resolution microscopy reveals ultra-low cd19 expression on myeloma cells that triggers elimination by cd19 car-t. Nature communications, 10(1):1-11, 2019. 
[144] Felix Wäldchen, Jan Schlegel, Ralph Götz, Michael Luciano, Martin Schnermann, Sören Doose, and Markus Sauer. Whole-cell imaging of plasma membrane receptors by 3d lattice light-sheet d storm. Nature communications, 11(1):1-6, 2020.

[145] Sebastian Strauss and Ralf Jungmann. Up to 100-fold speed-up and multiplexing in optimized dna-paint. Nature Methods, 17(8):789-791, 2020.

[146] Ralf Jungmann, Maier S Avendaño, Johannes B Woehrstein, Mingjie Dai, William M Shih, and Peng Yin. Multiplexed 3d cellular super-resolution imaging with dna-paint and exchange-paint. Nature methods, 11(3):313-318, 2014.

[147] Johannes Stein, Florian Stehr, Patrick Schueler, Philipp Blumhardt, Florian Schueder, Jonas Mücksch, Ralf Jungmann, and Petra Schwille. Toward absolute molecular numbers in dna-paint. Nano letters, 19(11):8182-8190, 2019.

[148] Sri Rama Prasanna Pavani, Michael A Thompson, Julie S Biteen, Samuel J Lord, Na Liu, Robert J Twieg, Rafael Piestun, and WE Moerner. Three-dimensional, single-molecule fluorescence imaging beyond the diffraction limit by using a doublehelix point spread function. Proceedings of the National Academy of Sciences, 106 (9):2995-2999, 2009.

[149] Laurent Holtzer, Tobias Meckel, and Thomas Schmidt. Nanometric threedimensional tracking of individual quantum dots in cells. Applied Physics Letters, 90(5):053902, 2007.

[150] Bence P Olveczky, N Periasamy, and AS Verkman. Mapping fluorophore distributions in three dimensions by quantitative multiple angle-total internal reflection fluorescence microscopy. Biophysical journal, 73(5):2836-2847, 1997.

[151] Nicolas Bourg, Céline Mayet, Guillaume Dupuis, Thomas Barroca, Pierre Bon, Sandrine Lécart, Emmanuel Fort, and Sandrine Lévêque-Fort. Direct optical nanoscopy with axially localized detection. Nature Photonics, 9(9):587-593, 2015.

[152] Siddharth Sivankutty, Thomas Barroca, Céline Mayet, Guillaume Dupuis, Emmanuel Fort, and Sandrine Lévêque-Fort. Confocal supercritical angle microscopy for cell membrane imaging. Optics letters, 39(3):555-558, 2014.

[153] Thomas Ruckstuhl and Dorinel Verdes. Supercritical angle fluorescence (saf) microscopy. Optics express, 12(18):4246-4254, 2004.

[154] Marcelina Cardoso Dos Santos, Régis Déturche, Cyrille Vézy, and Rodolphe Jaffiol. Topography of cells revealed by variable-angle total internal reflection fluorescence microscopy. Biophysical journal, 111(6):1316-1327, 2016. 
[155] Dinah Loerke, Walter Stühmer, and Martin Oheim. Quantifying axial secretorygranule motion with variable-angle evanescent-field excitation. Journal of neuroscience methods, 119(1):65-73, 2002.

[156] Jeffrey S Burmeister, George A Truskey, and William M Reichert. Quantitative analysis of variable-angle total internal reflection fluorescence microscopy (va-tirfm) of cell/substrate contacts. Journal of microscopy, 173(1):39-51, 1994.

[157] Christian M Winterflood, Thomas Ruckstuhl, Dorinel Verdes, and Stefan Seeger. Nanometer axial resolution by three-dimensional supercritical angle fluorescence microscopy. Physical review letters, 105(10):108103, 2010.

[158] Joran Deschamps, Markus Mund, and Jonas Ries. 3d superresolution microscopy by supercritical angle detection. Optics Express, 22(23):29081-29091, 2014.

[159] Narain Karedla, Alexey I Chizhik, Ingo Gregor, Anna M Chizhik, Olaf Schulz, and Jörg Enderlein. Single-molecule metal-induced energy transfer (smmiet): Resolving nanometer distances at the single-molecule level. ChemPhysChem, 15(4):705-711, 2014.

[160] RR Chance, A Prock, and R Silbey. Molecular fluorescence and energy transfer near interfaces. Adv. Chem. Phys, 37:1-65, 1978.

[161] Thilo Baronsky, Daja Ruhlandt, Bastian Rouven Brückner, Jonas Schäfer, Narain Karedla, Sebastian Isbaner, Dirk Hähnel, Ingo Gregor, Jörg Enderlein, Andreas Janshoff, et al. Cell-substrate dynamics of the epithelial-to-mesenchymal transition. Nano Letters, 17(5):3320-3326, 2017.

[162] Anna M Chizhik, Daja Ruhlandt, Janine Pfaff, Narain Karedla, Alexey I Chizhik, Ingo Gregor, Ralph H Kehlenbach, and Jörg Enderlein. Three-dimensional reconstruction of nuclear envelope architecture using dual-color metal-induced energy transfer imaging. ACS nano, 11(12):11839-11846, 2017.

[163] Anna M Chizhik, Carina Wollnik, Daja Ruhlandt, Narain Karedla, Alexey I Chizhik, Lara Hauke, Dirk Hähnel, Ingo Gregor, Jörg Enderlein, and Florian Rehfeldt. Dual-color metal-induced and förster resonance energy transfer for cell nanoscopy. Molecular biology of the cell, 29(7):846-851, 2018.

[164] Sebastian Isbaner, Narain Karedla, Izabela Kaminska, Daja Ruhlandt, Mario Raab, Johann Bohlen, Alexey Chizhik, Ingo Gregor, Philip Tinnefeld, Jörg Enderlein, et al. Axial colocalization of single molecules with nanometer accuracy using metalinduced energy transfer. Nano letters, 18(4):2616-2622, 2018. 
[165] Sergi Padilla-Parra, Nicolas Audugé, Maïté Coppey-Moisan, and Marc Tramier. Dual-color fluorescence lifetime correlation spectroscopy to quantify protein-protein interactions in live cell. Microscopy research and technique, 74(8):788-793, 2011.

[166] Jiji Chen and Joseph Irudayaraj. Fluorescence lifetime cross correlation spectroscopy resolves egfr and antagonist interaction in live cells. Analytical chemistry, 82(15):6415-6421, 2010.

[167] Krishanu Ray, Jian Zhang, and Joseph R Lakowicz. Fluorescence lifetime correlation spectroscopic study of fluorophore-labeled silver nanoparticles. Analytical chemistry, 80(19):7313-7318, 2008.

[168] Aleš Benda, Veronika Fagul'ová, Alexander Deyneka, Joerg Enderlein, and Martin Hof. Fluorescence lifetime correlation spectroscopy combined with lifetime tuning: New perspectives in supported phospholipid bilayer research. Langmuir, 22(23): 9580-9585, 2006.

[169] Jana Humpolíčková, Aleš Benda, Jan Sỳkora, Radek Macháň, Teresa Kral, Barbara Gasinska, Joerg Enderlein, and Martin Hof. Equilibrium dynamics of spermineinduced plasmid dna condensation revealed by fluorescence lifetime correlation spectroscopy. Biophysical journal, 94(3):L17-L19, 2008.

[170] Suren Felekyan, Stanislav Kalinin, Hugo Sanabria, Alessandro Valeri, and Claus AM Seidel. Filtered fcs: Species auto-and cross-correlation functions highlight binding and dynamics in biomolecules. ChemPhysChem, 13(4):1036-1053, 2012.

[171] Takuhiro Otosu and Shoichi Yamaguchi. Two-dimensional fluorescence lifetime correlation spectroscopy: Concepts and applications. Molecules, 23(11):2972, 2018.

[172] Amal Kasry, Ali A Ardakani, George S Tulevski, Bernhard Menges, Matthew Copel, and Libor Vyklicky. Highly efficient fluorescence quenching with graphene. The Journal of Physical Chemistry C, 116(4):2858-2862, 2012.

[173] Arindam Ghosh, Jörg Enderlein, and Eugenia Butkevich. Dimerization of human drebrin-like protein governs its biological activity. Biochemistry, 59(16):1553-1558, 2020.

[174] Steffen Mühle, Man Zhou, Arindam Ghosh, and Jörg Enderlein. Loop formation and translational diffusion of intrinsically disordered proteins. Physical Review E, 100(5):052405, 2019. 
[175] Ingo Gregor and Jörg Enderlein. Time-resolved methods in biophysics. 3. fluorescence lifetime correlation spectroscopy. Photochemical \& Photobiological Sciences, 6(1):13-18, 2007.

[176] Zdeněk Petrášek and Petra Schwille. Precise measurement of diffusion coefficients using scanning fluorescence correlation spectroscopy. Biophysical journal, 94(4): 1437-1448, 2008.

[177] Jonas Ries, Salvatore Chiantia, and Petra Schwille. Accurate determination of membrane dynamics with line-scan fcs. Biophysical journal, 96(5):1999-2008, 2009.

[178] Jan Christoph Thiele, Dominic Helmerich, Nazar Oleksiievets, Roman Tsukanov, Eugenia Butkevich, Markus Sauer, Oleksii Nevskyi, and Joerg Enderlein. Confocal laser-scanning fluorescence-lifetime single-molecule localisation microscopy. bioRxiv, 2020.

[179] Nazar Oleksiievets, Jan Christoph Thiele, André Weber, Ingo Gregor, Oleksii Nevskyi, Sebastian Isbaner, Roman Tsukanov, and Jörg Enderlein. Wide-field fluorescence lifetime imaging of single molecules. The Journal of Physical Chemistry A, 124(17):3494-3500, 2020.

[180] William Loomis. Dictyostelium discoideum: a developmental system. Elsevier, 2012. 
Curriculum Vitae 


\section{Arindam Ghosh}

Ph.D. candidate, AG Enderlein

III. Institute of Physics, Biophysics

International Max Planck Research School for

Physics of Biological and Complex Systems

(IMPRS - PBCS)

University of Göttingen

Friedrich Hund Platz 1, 37077 Göttingen, Germany

E-mail - arindam.ghosh@phys.uni-goettingen.de

Phone - +49-(0)551-39 26909

Date of Birth: $15^{\text {th }}$ November 1992, Place: Kolkata, India

\section{Research Focus}

Single molecule spectroscopy and super-resolution microscopy: from method development to applications in life sciences, fast conformational dynamics in biomolecules, membrane biophysics.

\section{Research Experience}

\section{- University of Göttingen}

Ph.D. student in the group of Jörg Enderlein (08/16 - present)

Thesis title: Single Molecule Fluorescence Spectroscopy and Imaging: Advanced Methods and Applications in Life Sciences.

- Indian Institute of Technology Bombay (IITB)

Masters' thesis: Studying in vitro Aggregation of Human Growth Hormone (07/15-05/16)

Summer project: Optimization of expression and purification protocol of doubly labeled human growth hormone using glucose as source of carbon. (05/15 - 07/15)

Other projects: Comparative study on Fructose 1, 6 bisphosphate Aldolase from various organisms from an evolutionary point of view using Bioinformatics tools (09/15 - 11/15)

Education

- University of Göttingen and IMPRS-PBCS, Göttingen, Germany (08/16 - present) Ph.D. candidate (degree expected by summer 2020)

- Indian Institute of Technology Bombay (IITB), Mumbai, India (07/14 - 05/16) Master of Science (M.Sc.) in Biotechnology, (Overall grade point average 8.05/10)

- Bidhan Chandra Krishi Viswavidyalaya (BCKV), Kalyani, India (08/10 - 06/14) Bachelor of Science (B.Sc.) in Agricultural Sciences (Honours) major module - Plant Biochemistry, (Overall grade point average 8.39/10) 
- Ghosh, A., Chizhik, A. I., Karedla, N., \& Enderlein, J. (2020). Graphene- and metal-induced energy transfer: From single-molecule imaging to live cell nanoscopy with (sub)-nanometer axial resolution. Nature Protocols (invited protocol in revision).

- Ghosh, A., Enderlein, J., \& Butkevich, E. (2019). Dimerization of human drebrin-like protein governs its biological activity. Biochemistry, 59 (16), 1553-1558.

- Ghosh, A., Sharma, A., Chizhik, A. I., Isbaner, S., Ruhlandt, D., Tsukanov, R., .. \& Enderlein, J. (2019). Graphene-based metal-induced energy transfer for sub-nanometre optical localization. Nature Photonics, 13(12), 860-865.

- Mühle, S., Zhou, M., Ghosh, A., \& Enderlein, J. (2019). Loop formation and translational diffusion of intrinsically disordered proteins. Physical Review E, 100(5), 052405.

- Ghosh, A., Karedla, N., Thiele, J. C., Gregor, I., \& Enderlein, J. (2018). Fluorescence lifetime correlation spectroscopy: Basics and applications. Methods, 140, 32-39.

- Ghosh, A., Isbaner, S., Veiga-Gutiérrez, M., Gregor, I., Enderlein, J., \& Karedla, N. (2017). Quantifying microsecond transition times using fluorescence lifetime correlation spectroscopy. The journal of physical chemistry letters, 8(24), 6022-6028.

\section{Conference and Seminar Oral and Poster Presentations}

- PicoQuant $25^{\text {th }}$ International Anniversary Workshop, Berlin, Germany, 09/19.

- Focus on Microscopy, London, U.K., 04/19.

- Molecular Biophysics Meeting, Hünfeld, Germany, 02/19.

- Membrane Imaging Workshop, SDU, Denmark, 12/18.

- Biophysics by the Sea, Mallorca, Spain, 10/18.

- Poster presentation: PicoQuant Workshop, Berlin, Germany, 09/18.

- Focus on Microscopy, Singapore, 04/18.

- $\quad$ SFB 860 Retreat, Hirschweg, Austria, 02/18.

- Quantitative BioImaging Conference (QBI) 2018, Göttingen, Germany, 01/18.

- Biophysics by the Sea, Mallorca, Spain, 10/17.

- Third Infinity Conference, Göttingen, Germany, 10/17.

- PicoQuant Workshop, Berlin, Germany, 09/17.

- Poster presentation: GGNB Science Day, Göttingen, Germany, 12/16. 
- Best student talk award at PicoQuant $25^{\text {th }}$ International Anniversary Workshop, Berlin, Germany, 09/2019.

- Merit scholarship provided by Department of Biotechnology (DBT), India during Masters' studies (2014 - 2016).

- University Merit scholarship during Bachelors’studies (2010 - 2014).

Teaching and Other Activities

- Teaching: methods course - 'Basics of fluorescence microscopy and spectroscopy in biophysics'

- Editor and author in annual graduate school newsletter GGNB Times 2018 issue

- Student representative, IMPRS-PBCS (2018 - 2019)

- Organization of 'Third Infinity Conference' 2017 